\title{
A MULTI-WAVE LONGITUDINAL EXAMINATION OF HOW STRENGTHS AND RISKS INFORM RISK ASSESSMENT AND TREATMENT PROFILES FOR JUSTICE-INVOLVED MEN AND WOMEN USING THE SERVICE PLANNING INSTRUMENT (SPIN)
}

\author{
by \\ Kayla A. Wanamaker \\ Dissertation submitted to \\ the Faculty of Graduate Studies and Research \\ in Partial Fulfillment of the requirements for the degree \\ Doctor of Philosophy \\ in \\ Psychology \\ Carleton University \\ Ottawa, Ontario \\ (C)2020
}

Kayla A. Wanamaker 


\title{
STRENGTHS, RISK MANAGEMENT, AND GENDER
}

\begin{abstract}
The examination of offender change on gender-informed dynamic strength and risk factors is limited. More research is needed focusing on the predictive nature of dynamic risk and strength score changes and whether there are gender differences or similarities between justice-involved men and women. Study 1 used a Canadian sample of 11,953 men and 2,877 women under community supervision with Service Planning Instrument (SPIn) re-assessment data (Orbis Partners, 2003). Using a retrospective, multi-wave longitudinal research design, hierarchical linear modeling (HLM) was conducted to assess patterns of change in total dynamic risk and strength scores across three to five assessment timepoints over a 30-month period. Following this, the change parameters (intercepts and slopes) from the HLM were incorporated into a series of regression models for men and women independently, linking change over time to four reoffending outcomes: technical violations, new charges, violent charges, and any negative outcome. Study 1 found that total dynamic risk scores decreased over time and total dynamic strength scores increased over time for both men and women, although the rate of change for strength and risk scores was minimal, occurring gradually over time. For men, change in total dynamic risk scores was predictive of all four outcomes, whereas for women, change in total dynamic risk scores was predictive of technical violations, new charges, and any negative outcome, but not violent charges. Change in total dynamic strength scores only predicted technical violations for both genders.
\end{abstract}

Using a sub-sample of SPIn assessments for 6,675 men and 1,684 women from Study 1, Study 2 examined typologies of justice-involved men and women comprised of gender-neutral and gender-responsive risk and strength factors, and whether these 


\section{STRENGTHS, RISK MANAGEMENT, AND GENDER}

typologies were stable over time. Specifically, latent profile analyses (LPAs) were conducted independently for men and women at three timepoints: Time $1=$ first assessment within 90 days of start of supervision, Time 2 = between 3 and 8 months post initial assessment, and Time $3=$ between 9 and 14 months post initial assessment. Subsequently, the relationship between typological membership and reoffending outcomes were examined. Three profiles consistently emerged at each timepoint for women: a low risk/low strength profile, a gender-responsive, low risk/high strength profile, and an aggressive, complex need/low strength profile, whereby women scored higher on both gender responsive needs and gender-neutral risk factors. Men were classified into five profiles: a low risk/low strength profile, an aggressive, complex need/low strength profile, a moderate risk/moderate strength profile, a low risk/high strength profile, and a low stability, complex need/low strength profile. Additionally, at Time 3, a sixth profile of men emerged labelled moderate complex need/low strength. Importantly, profiles characterized as aggressive with complex needs had the highest rate of reoffending for both genders.

Findings indicate that both men and women exhibit changes in total dynamic risk and strength scores over time, albeit minimally. While change scores on total dynamic risk are predictive of reoffending outcomes, more research assessing the dynamic nature of strengths is needed to further inform risk assessment protocols for technical violations. Results indicate more similarities than differences in typological structure of men and women, although heterogeneity for men increased over time. Both men and women at greater risk to fail were characterized by high scores on aggression and complex needs, which can help further inform treatment targets and case management planning. 


\section{STRENGTHS, RISK MANAGEMENT, AND GENDER}

\section{Acknowledgements}

First and foremost, I would like to thank my supervisor, Dr. Shelley Brown, for all her support, guidance, motivation, enthusiasm, and encouragement over the last eight years. I have learned so much from you throughout my time at Carleton; you have been an incredible mentor and a true inspiration. I look forward to continue collaborating with you in the future.

I would like to thank my committee members, Dr. Adelle Forth and Dr. Natalie Jones. Your thoughtful feedback and helpful suggestions have undoubtedly improved my research. I would also like to thank my internal examiner, Dr. Diana Majury, and my external examiner, Dr. Mark Olver, for volunteering their time to read my document, offer feedback, and pose challenging and thought-provoking questions at my defence.

I would like to express thanks to my lab-mates (past and present) from the Gender and Crime lab who have offered words of encouragement, guidance, and friendship over the years (especially Dr. Terri Scott, Dr. Leigh Greiner, Cassie Conley, Megan Wagstaff, Shreena Thapa, and Allysa Czerwinsky). I appreciate each of you!

A huge thank you goes out to Dr. David Robinson and Orbis Partners Inc. for providing me with the opportunity to conduct my research using their risk, strength, and case management planning tool, the Service Planning Instrument (SPIn). I would like to express my sincere gratitude to Alberta Solicitor General and Public Security for providing the SPIn and recidivism data — without this rich dataset, this research would not have been possible.

To my friends and colleagues - you each have played a unique and integral role in helping me complete my doctoral degree. To my colleague, co-author, conference 


\section{STRENGTHS, RISK MANAGEMENT, AND GENDER}

roommate, and good friend, Leticia Gutierrez. You continuously inspire me to be the best person I can be. I want to thank you for being a sounding board and always offering words of reassurance and unwavering support when I need it most. Your friendship and kindness means so much to me and I look forward to many more adventures with you in the future.

To my friends and colleagues, Drs. Kaitlyn Wardrop and Nick Chadwick-I want to thank you both for listening to my concerns, answering my questions, and being there for me when I needed it. I am so thankful to have gone through this $\mathrm{PhD}$ journey with you, and I look forward to continue working (and potentially collaborating) with you. To Drs. Laura Dunbar and Rebecca Mugford-your kind words and positivity have kept me going and I am truly grateful.

To my family and friends - especially mom, dad, Amanda, Warren, and Alyssa_I appreciate you all for supporting me over the years and for being incredibly understanding when I had to miss events or family get-togethers. I could not have asked for better parents, sisters, or brother. I love you all so much and I could not have done this without you guys!

Finally, to my absolute best friend and loving husband, Matt. I knew these past few years have not been easy; you have had to deal with my stress and frustrations, have had to take on additional work around the house, and have had to attend many events on your own (so that I could work on my dissertation). You have never complained, and continuously encouraged me to keep going and offering words of support. You mean the world to me and I can't wait to start the next chapter of our lives together. I love you and appreciate you more than you know. 


\section{Table of Contents}

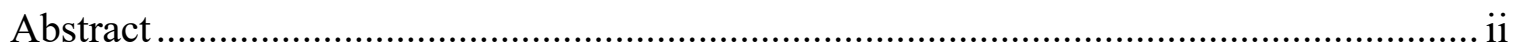

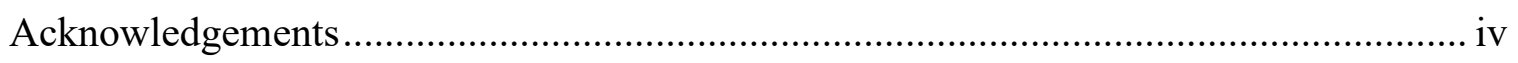

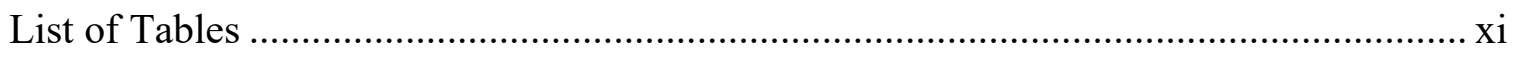

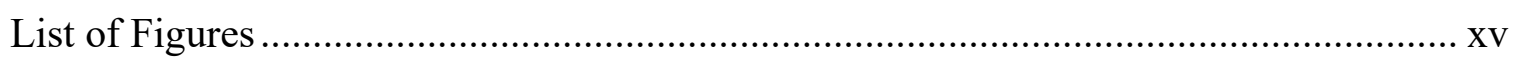

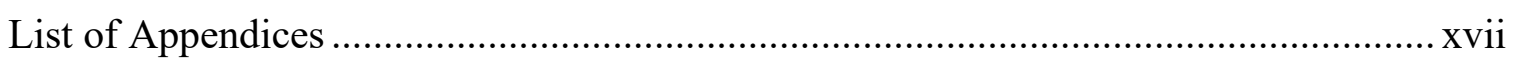

Chapter 1: General Introduction - Theoretical Underpinnings ...................................... 1

Chapter 2: Detailed Literature Review - Theoretical Foundation................................... 7

General Personality and Cognitive Social Learning Theory....................................... 7

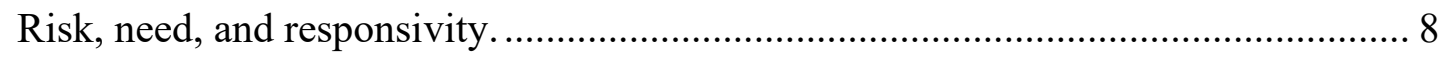

Gender-Responsive Pathways Theory .................................................................. 13

Pathways theory: Empirical support and critiques ............................................ 16

Strengths-Based Correctional Perspective ......................................................... 20

Definitional Caveats_-Strengths, Promotive, and Protective Factors ......................... 21

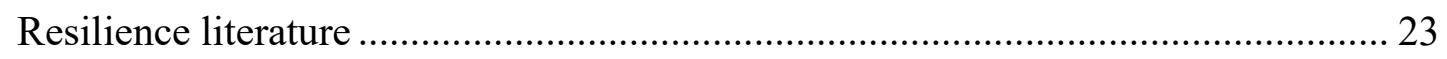

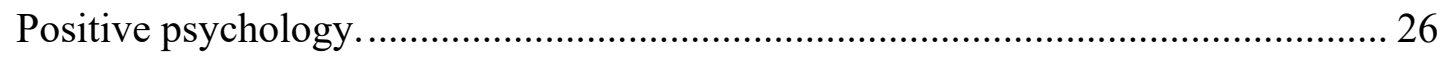

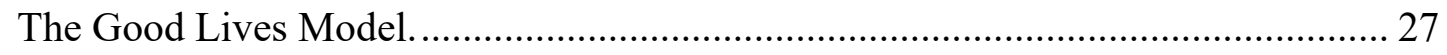

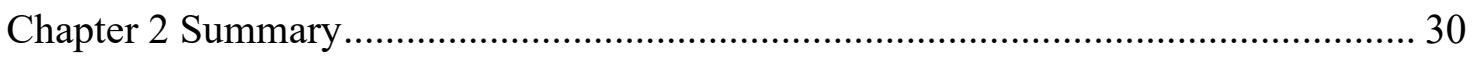

Making the Case for Theoretical Integration ....................................................... 31

Chapter 3. Study 1: Assessing Patterns of Change in Dynamic Risk and Dynamic Strength Factors and the Relationship to Recidivism for Men and Women.................... 35

Multi-wave Assessment of Dynamic Risk ................................................................. 37

Multi-wave Studies Assessing Dynamic Risk and Justice-Involved Men................... 38

Multi-wave Studies Assessing Dynamic Risk and Justice-Involved Women ............. 40

Multi-wave Studies Assessing Dynamic Strengths............................................... 41

Using the Service Planning Instrument to Assess Change Over time......................... 43

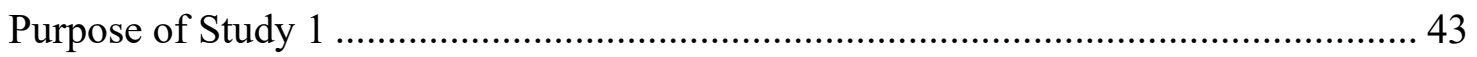

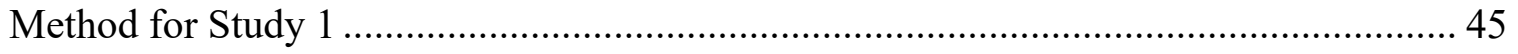

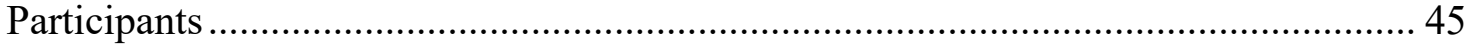

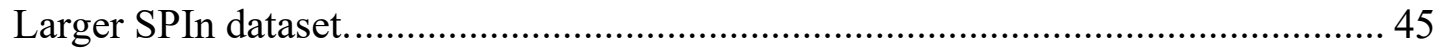

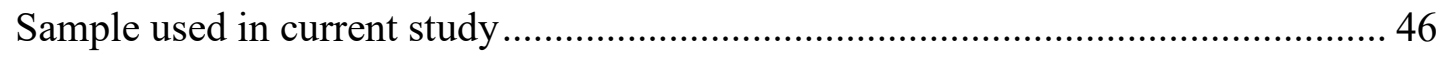

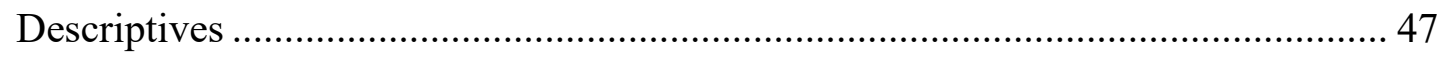




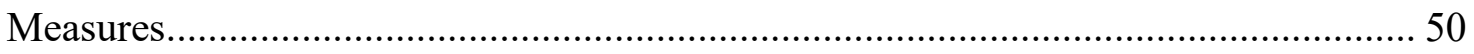

The Service Planning Instrument (SPIn) - Full Assessment..................................... 50

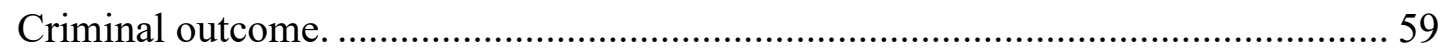

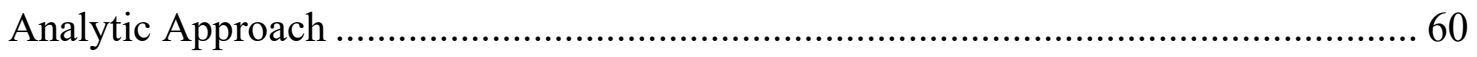

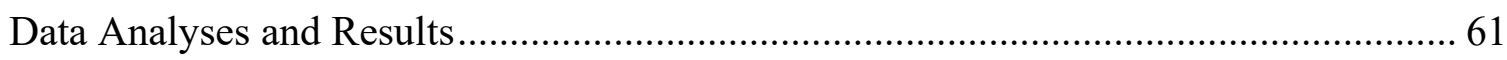

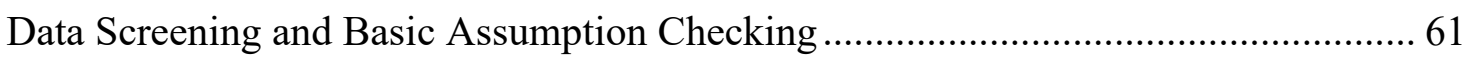

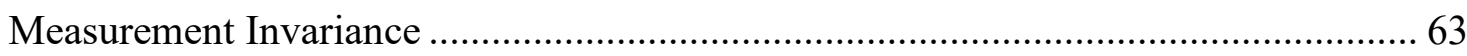

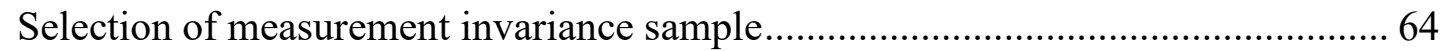

Assumption checking for exploratory factor analysis............................................. 68

Exploratory Factor Analysis (EFA)....................................................................... 70

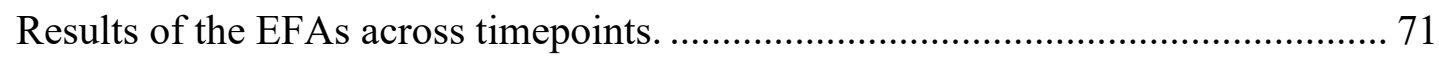

Exploratory Structural Equation Modeling (ESEM) ………………………........... 74

Patterns of change: Description of Analyses.......................................................... 78

Hierarchical linear growth modeling................................................................... 78

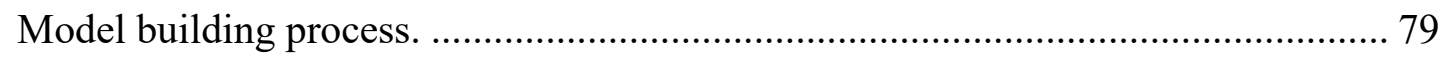

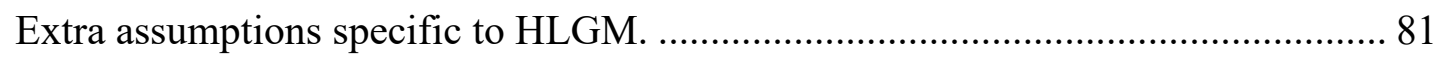

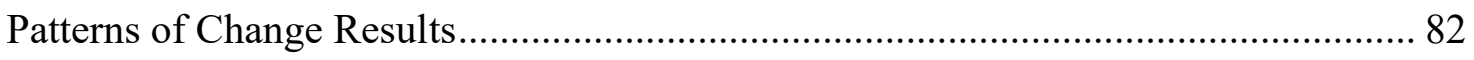

Patterns of change results: Women and total dynamic risk scores............................ 82

Patterns of change results: Women and total dynamic strength scores..................... 89

Patterns of change results: Men and total dynamic risk scores................................. 95

Patterns of change results: Men and dynamic strength scores. ............................... 100

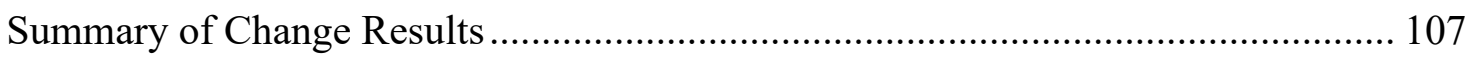

Using Offender Change in Dynamic Strengths and Risks to Predict Outcome .......... 109

Criminal outcome for women and men. .......................................................... 110

Using change to predict criminal outcomes for women.......................................... 111

Logistic regression results - Total dynamic risk scores......................................... 112

Logistic regression results - Total dynamic strength scores. ................................... 115

Using change to predict criminal outcomes for men............................................. 117

Logistic regression results - Total dynamic risk scores......................................... 119

Logistic regression results - Total dynamic strength scores.................................. 122

Summary of Prediction Results........................................................................... 124

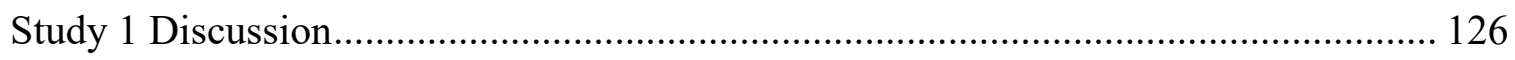


Patterns of Change in SPIn-Assessed Total Dynamic Risk Scores ............................ 127

Patterns of Change in SPIn-Assessed Total Dynamic Strength Scores ..................... 130

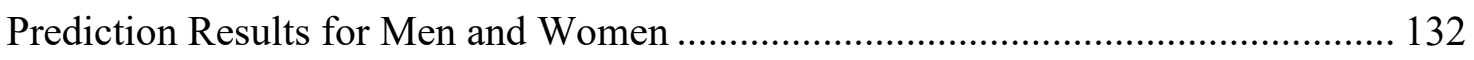

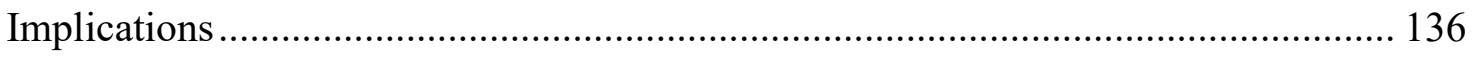

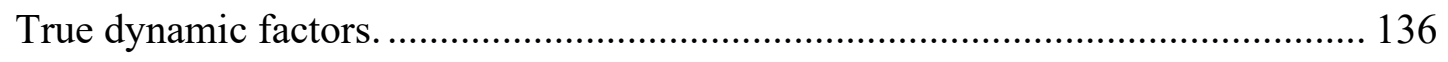

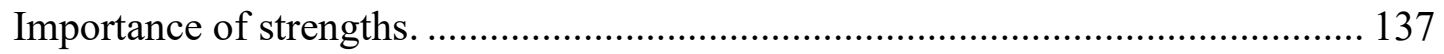

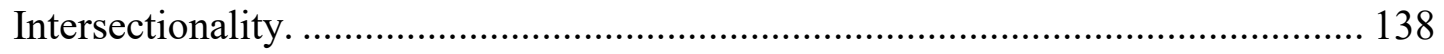

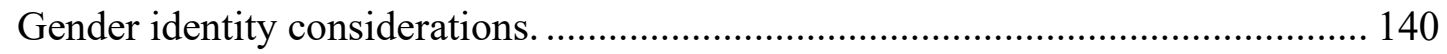

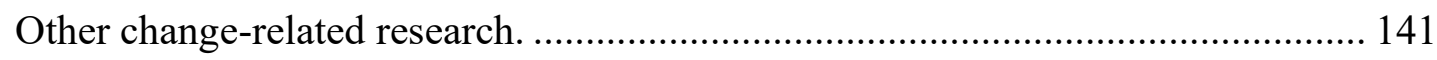

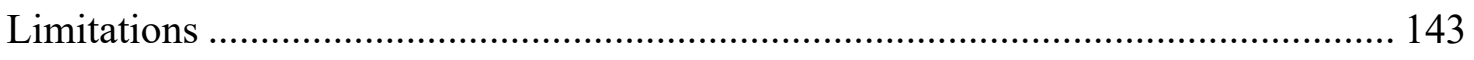

Loss of data and generalizability concerns.......................................................... 143

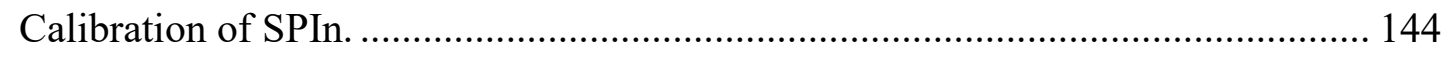

Psychometric data and ability to assess change...................................................... 145

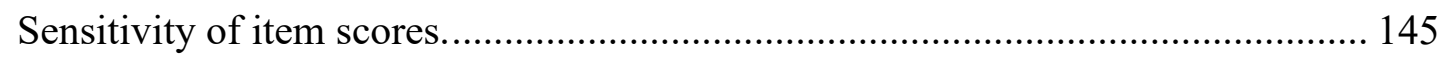

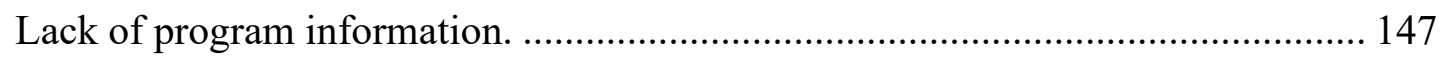

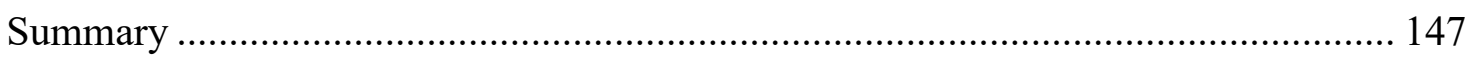

Chapter 4 Study 2: Building a Holistic Typology to Inform Service Delivery for Men and Women on Community Supervision......................................................................... 149

Trajectory-Based Typology Research .................................................................. 150

Gender Neutral Risk and Need Based Typology Research......................................... 152

Justice-Involved Female Typology Research ……………………........................ 154

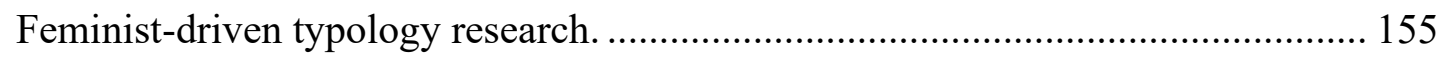

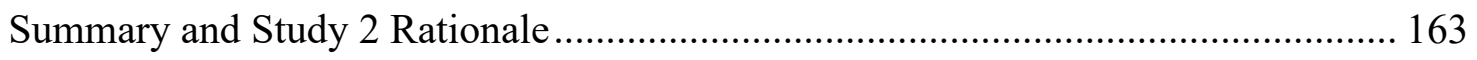

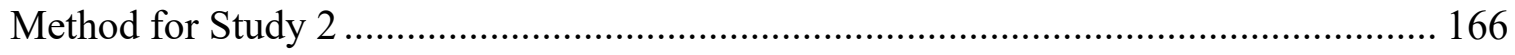

Participants and Research Design .................................................................... 166

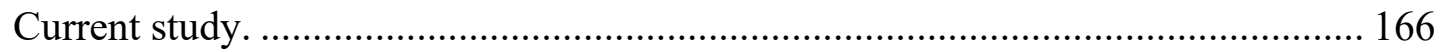

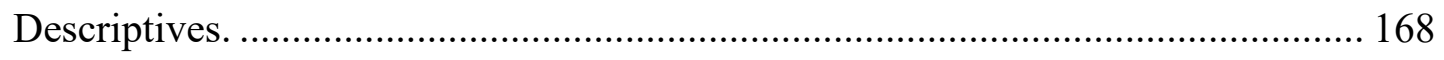

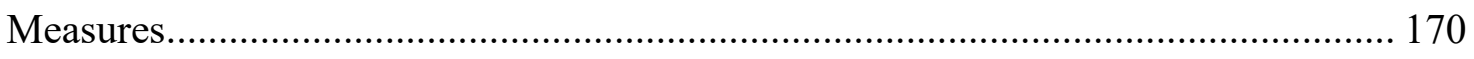

The Service Planning Instrument (SPIn) - Full Assessment.................................... 170

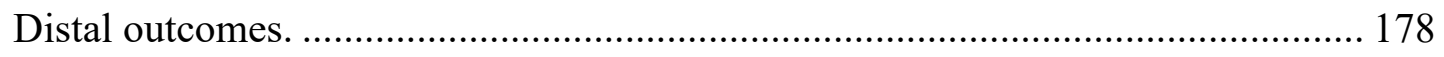

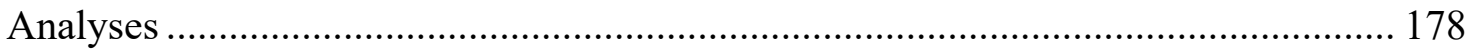

Latent profile analysis (LPA) and latent transition analysis (LTA) ........................ 178

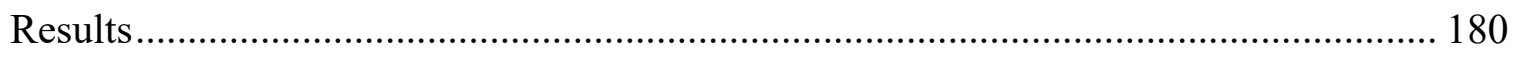




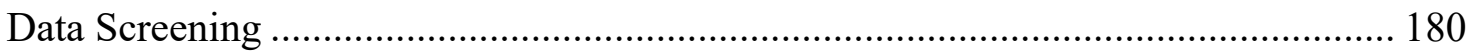

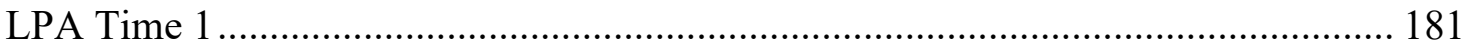

Time 1 profile descriptions for women. .............................................................. 183

Time 1 profile descriptions for men. ................................................................ 187

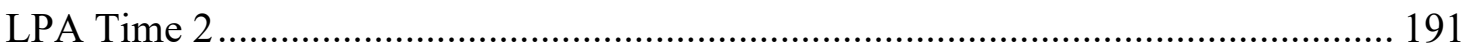

Time 2 profile descriptions for women and men................................................. 192

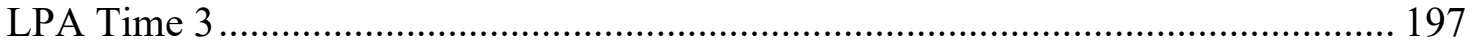

Time 3 profile descriptions for women. ............................................................. 198

Time 3 profile descriptions for men. ..................................................................... 201

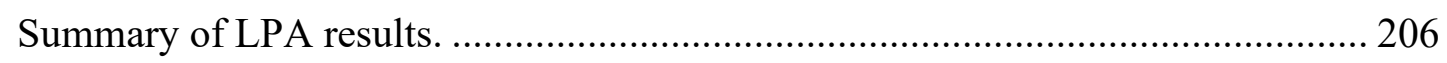

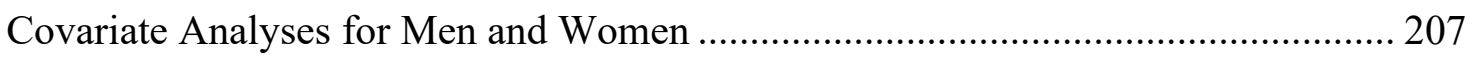

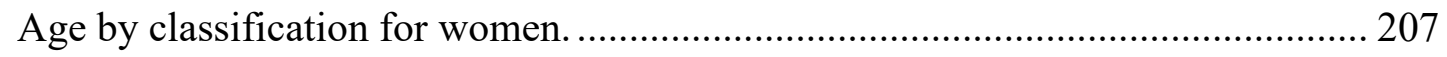

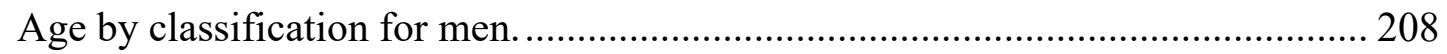

Total static risk scores by classification for women.............................................. 208

Total static risk scores by classification for men................................................... 209

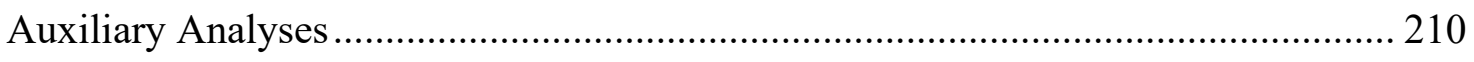

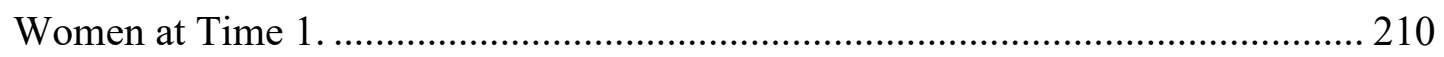

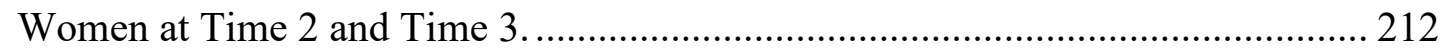

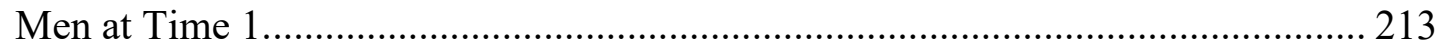

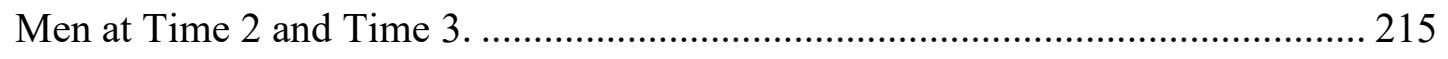

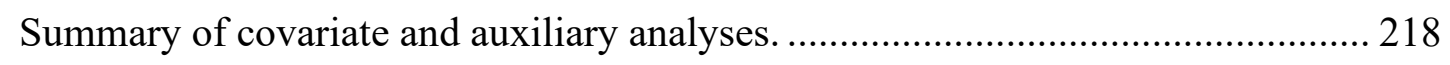

Latent Transitional Probabilities for Women........................................................ 220

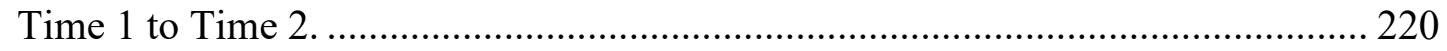

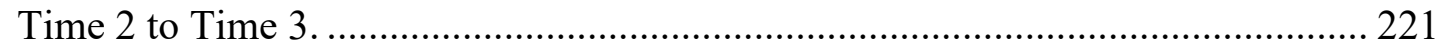

Latent Transitional Probabilities for Men ................................................................ 223

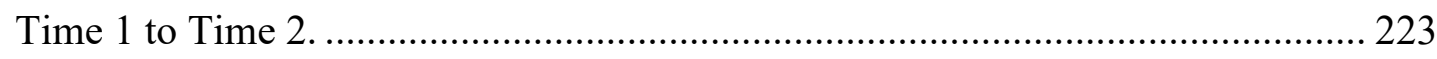

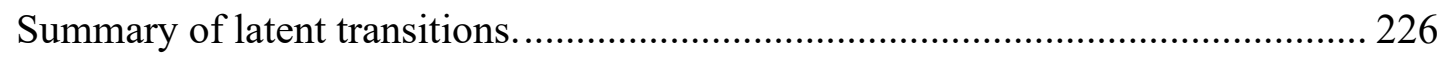

Typological Structure and Criminal Outcomes....................................................... 226

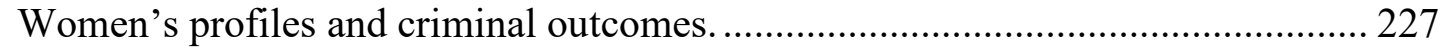

Men's profiles and criminal outcomes. ……..................................................... 228

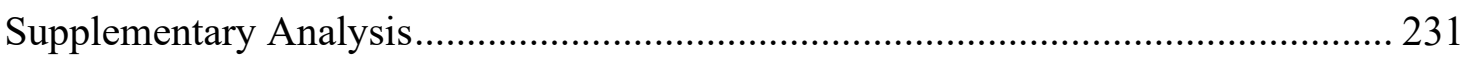

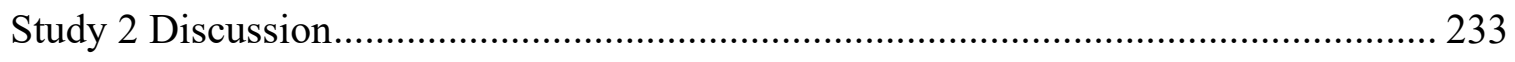




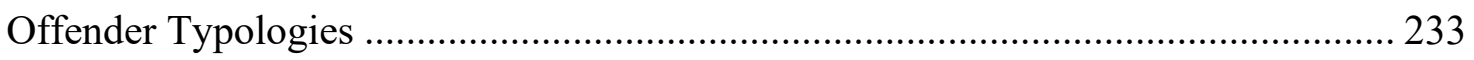

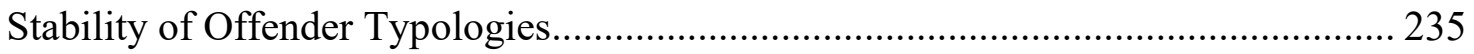

Gender and Typological Membership................................................................... 236

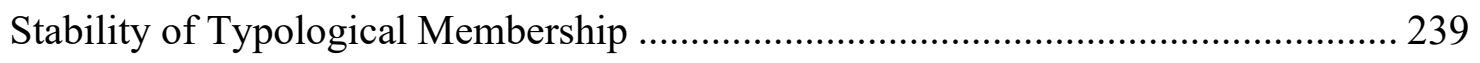

Typological Membership and Criminal Outcomes .................................................. 241

Indigenous Men and Women and Profile Membership ............................................. 243

Limitations and Directions for Future Research .................................................. 244

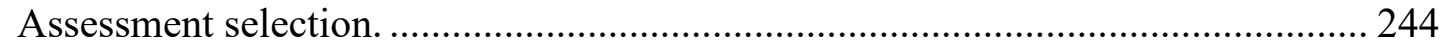

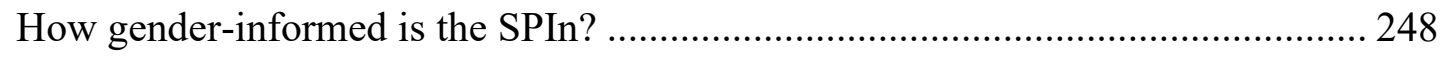

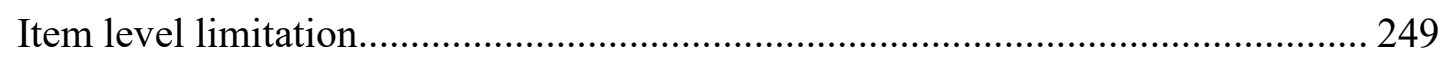

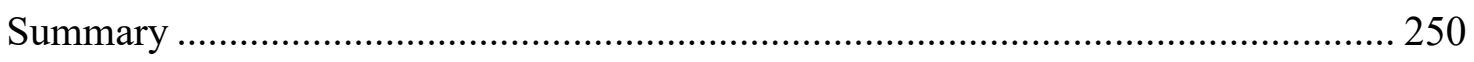

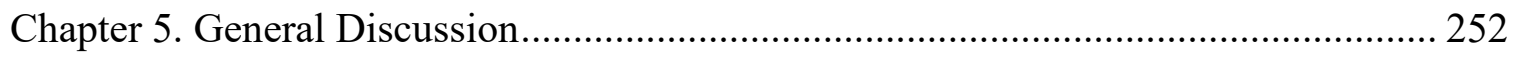

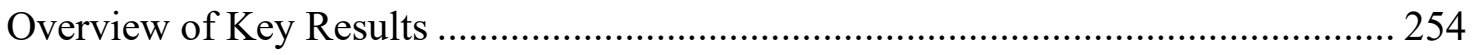

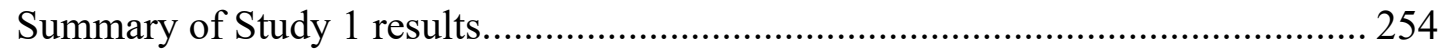

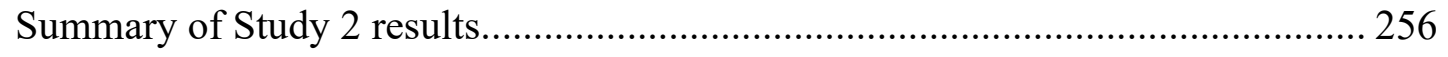

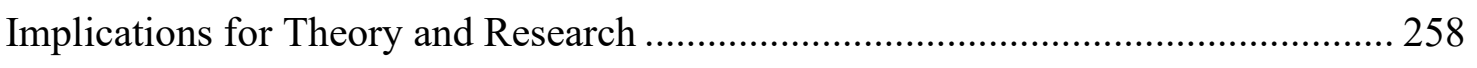

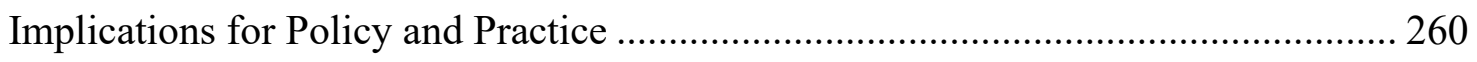

Limitations and Directions for Future Research ...................................................... 262

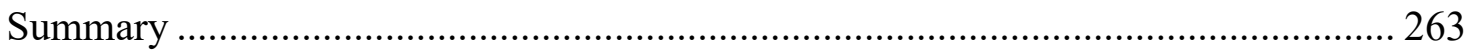

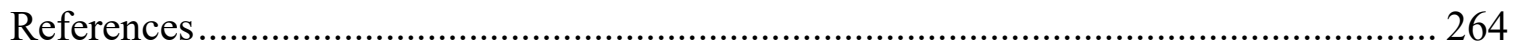




\section{List of Tables}

Table 1. Descriptive Information for Study 1, Split by Gender. 49

Table 2. Score Ranges Corresponding to Classification Levels on Full Assessment Total Dynamic Risk and Strength Scores........................................................................ 56

Table 3. Missing Data on Full SPIn Assessments Across Five Timepoints .................... 62

Table 4. Descriptive Information of Measurement Invariance Sample of Men and Women 67

Table 5. Correlations between SPIn Full Assessment Total Static Risk, Dynamic Risk and Dynamic Strength Score at Each Timepoint ......................................................... 70

Table 6. Decision Rules for Factor Retention and Structure Fit at Each Time for Men and

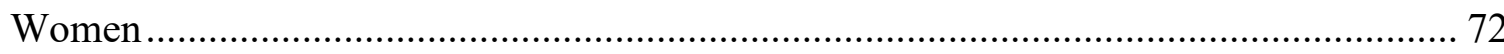

Table 7. Model Fit Indices to Examine Factor Structure Fit at Each Time for Men and Women..... 73

Table 8. Model Fit Indices and Nested Model Comparisons for Testing Configural and Scalar Measurement Invariance for Men. 76

Table 9. Model Fit Indices and Nested Model Comparisons for Testing Configural and Scalar Measurement Invariance for Women. 77

Table 10. Two-level Linear Growth Models Examining Changes in Total Dynamic Risk Scores Over Time for Women $(\mathrm{N}=2,877)$ 85

Table 11. Comparison of Pseudo R2 Statistics between Unconditional and Conditional Growth Models for Women-Total Dynamic Risk Scores 88

Table 12. Two-level Linear Growth Models Examining Changes in Total Dynamic Strength Scores Over Time for Women $(\mathrm{N}=2,877)$......

Table 13. Comparison of Pseudo $\mathrm{R}^{2}$ Statistics between Unconditional and Conditional Growth Models for Women-Total Dynamic Strength Scores.

Table 14. Two-level Linear Growth Models Examining Changes in Total Dynamic Risk Scores Over Time for Men $(\mathrm{N}=11,953)$. 97

Table 15. Comparison of Pseudo $\mathrm{R}^{2}$ Statistics between Unconditional and Conditional Growth Models for Men-Total Dynamic Risk Scores . 100

Table 16. Two-level Linear Growth Models Examining Changes in Total Dynamic Strength Scores Over Time for Men $(\mathrm{N}=11,953)$..... 103

Table 17. Comparison of Pseudo $\mathrm{R}^{2}$ Statistics between Unconditional and Conditional Growth Models for Men-Total Dynamic Strength Scores .... 106 


\section{STRENGTHS, RISK MANAGEMENT, AND GENDER}

Table 18. Summary and Comparison of Results for the Dynamic Risk and Strength

Models for Women and Men

Table 19. Frequency of Men and Women who Reoffended Over a Three-Year Fixed Follow-up

Table 20. Average Rate of Change and Initial Total Dynamic Risk and Strength Scores for Women

Table 21. Logistic Regression Results: Predicting Criminal Outcome using Change in Total Dynamic Risk Scores for Women

Table 22. Logistic Regression Results: Predicting Criminal Outcome using Change in Total Dynamic Strength Scores for Women.

Table 23. Comparing the Subsample to the Full Sample of Justice-Involved Men used for Analyses.....

Table 24. Average Rate of Change and Initial Dynamic Risk and Strength Scores for Men

Table 25. Logistic Regression Results: Predicting Criminal Outcome using Change in Total Dynamic Risk Scores for Men

Table 26. Logistic Regression Results: Predicting Criminal Outcome using Change in Total Dynamic Strength Scores for Men ....

Table 27. Summary and Comparison of Prediction Results of Total Dynamic Risk and Strength Scores for Men and Women.....

Table 28. Summary of Brennan et al. (2008) Typologies........................................... 153

Table 29. Summary of Daly’s (1992 \& 1994) Pathways into Crime............................. 156

Table 30. Summary of Dehart's (2018) Typologies of Justice-Involved Women.......... 159

Table 31. Summary of Perkins' (2010) Classes of Justice-Involved Men and Women . 161

Table 32. Descriptive Information for Men and Women and Gender Differences ........ 169

Table 33. Relative Fit Statistics for Time 1 for Men and Women, Independently......... 182

Table 34. Posterior Profile Membership Probabilities for Time 1: Men and Women.... 183

Table 35. Means for Each of the Risk and Strength Domains across the Three Profiles of Women at Time 1

Table 36. Means for Each of the Risk and Strength Domains across the Five Profiles of

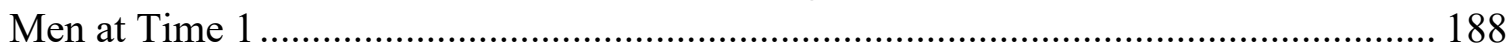

Table 37. Relative Fit Statistics for Time 2 for Men and Women, Independently 191 


\section{STRENGTHS, RISK MANAGEMENT, AND GENDER}

Table 38. Posterior Profile Membership Probabilities for Time 2: Men and Women.... 192

Table 39. Means for Each of the Risk and Strength Domains across the Three Profiles of

Women at Time 2

Table 40. Means for Each of the Risk and Strength Domains across the Three Profiles of Men at Time 2

Table 41. Relative Fit Statistics for Time 3 for Men and Women, Independently

Table 42. Posterior Profile Membership Probabilities for Time 3: Men and Women.... 198

Table 43. Means for Each of the Risk and Strength Domains across the Three Profiles of Women at Time 3

Table 44. Means for Each of the Risk and Strength Domains across the Six Profiles of

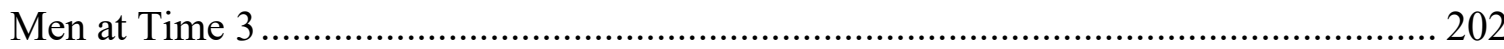

Table 45. Mean Age for Each Profile Across Timepoints for Women ......................... 207

Table 46. Mean Age for Each Profile Across Timepoints for Men ............................... 208

Table 47. Mean Total Static Risk Scores for Each Profile Across Timepoints for Women.....

Table 48. Mean Total Static Risk Score for Each Profile Across Timepoints for Men

Table 49. Time 1 Proportions of Indigenous and Non-Indigenous Women in Each

Profile.

Table 50. Time 2 and 3 Proportions of Indigenous and Non-Indigenous Women in Each Profile.

Table 51. Time 1 Proportions of Indigenous and Non-Indigenous Men in Each Profile

Table 52. Time 2 and 3 Proportions of Indigenous and Non-Indigenous Men in Each Profile.....

Table 53. Transitional Probabilities between Profiles from Time 1 to Time 2 for Women.

Table 54. Profile Transitions for Women from Time 1 to Time 2

Table 55. Transitional Probabilities between Profiles from Time 2 to Time 3 for Women

Table 56. Profile Transitions for Women from Time 2 to Time 3

Table 57. Transitional Probabilities between Profiles from Time 1 to Time 2 for Men 
Table 58. Profile Transitions from Time 1 to Time 2 for Men ..................................... 225

Table 59. Proportions of Women who Committed Each Outcomes Across Profiles at

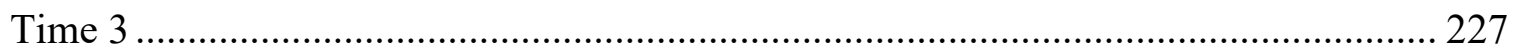

Table 60. Profile Comparisons of Various Criminal Outcomes for Women .................. 228

Table 61. Proportions of Men Convicted of Each Criminal Outcome Across Profiles at

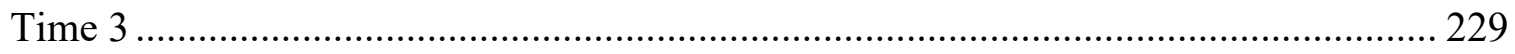

Table 62. Profile Comparisons of Various Criminal Outcomes for Men ...................... 230 


\section{STRENGTHS, RISK MANAGEMENT, AND GENDER}

\section{List of Figures}

Figure 1. Participant flow chart for study 1.

Figure 2. Breakdown of SPIn full assessment dynamic and static risk and strength

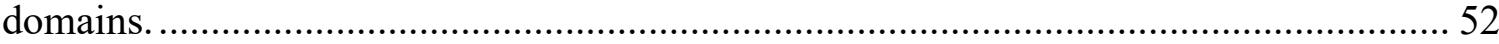

Figure 3. Example of a SPIn item (current intimate relationship) ....................53

Figure 4. Women's change in total dynamic risk scores over time as a function of total static risk score.

Figure 5. Individual change on total dynamic risk scores (predicted) across time in months for women.

Figure 6. Women's change in total dynamic strength scores over time as a function of Indigenous status.

Figure 7. Individual change on total dynamic strength scores (predicted) across time in months for women.

Figure 8. Men's change in total dynamic risk scores over time as a function of Indigenous status.

Figure 9. Individual change on total dynamic risk scores (predicted) across time in months for men.

Figure 10. Men's change in dynamic strength scores over time as a function of Indigenous status.

Figure 11. Individual change on total dynamic strength scores (predicted) across time in months for men. 105

Figure 12. Participant flow chart for Study 2.

Figure 13. Standardized risk and strength domain scores for the three profiles of women at Time 1.

Figure 14. Standardized risk and strength domain scores for the five profiles of men at Time 1.

Figure 15. Standardized risk and strength domain scores for the three profiles of women at Time 2.

Figure 16. Standardized risk and strength domain scores for the five profiles of men at Time 2.

Figure 17. Standardized risk and strength domain scores for the three profiles of women at Time 3. 200

Figure 18. Standardized risk and strength domain scores for the six profiles of men at Time 3. 


\section{STRENGTHS, RISK MANAGEMENT, AND GENDER}

Figure 19. Proportion of Indigenous and non-Indigenous women in each profile at

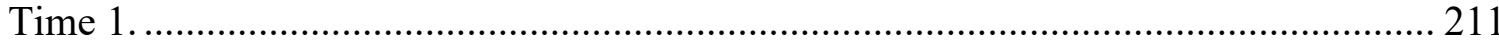

Figure 20. Proportions of Indigenous and non-Indigenous men in each profile at Time 1.

Figure 21. Proportions of Indigenous and non-Indigenous men in each profile at

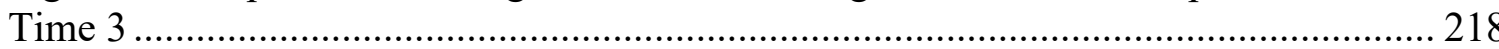


STRENGTHS, RISK MANAGEMENT, AND GENDER

\section{List of Appendices}

Appendix A. Detailed Description of the Service Planning Instrument (SPIn; Orbis

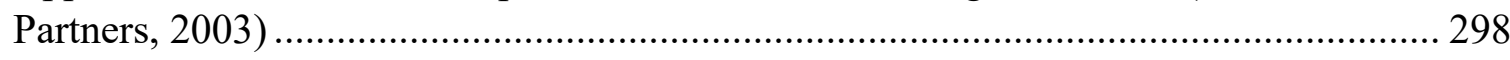

Appendix B. Internal Consistency of the Dynamic Risk and Strength Domains ........... 307

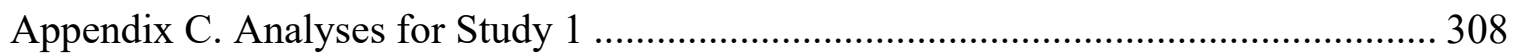

Appendix D. Means, Standard Deviations, and Correlations between SPIn Dynamic Domains for Each of the Three Timepoints used for Measurement Invariance for Each Gender

Appendix E. Varimax Rotated Loadings and Standard Errors across Timepoints and For Each Gender.

Appendix F. Measurement Invariance Results for 2-Factor Structure of the SPIn used in Practice Over Three Timepoints for Men and Women........................................... 317

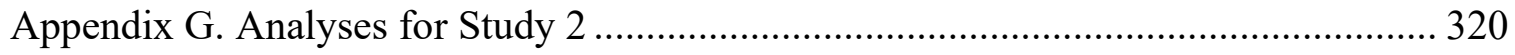

Appendix H. Means, Standard Deviations and Ranges for Variables Included in LPAs at Each Timepoint for Each Gender ................................................................. 321

Appendix I. Supplemental Tables for Covariates (Age and Total Static Risk Scores) For Women and Men at Each Timepoint ................................................................. 324

Appendix J. Supplemental Tables for the Auxiliary Variable (Indigenous Status) for

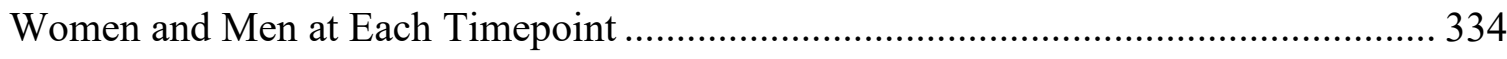

Appendix K. Supplementary LPA Analyses - Results for Full Sample of Men and Women from 3-Year Fixed-Follow-up Cohort with Initial SPIn Full Assessment ........ 337 
A multi-wave longitudinal examination of how strengths and risks inform risk assessment and treatment profiles for justice-involved men and women using the Service Planning Instrument (SPIn)

\section{Chapter 1: General Introduction - Theoretical Underpinnings}

The criminal justice field has predominately been influenced by traditional, mainstream correctional research (Andrews et al., 2012; Bonta \& Andrews, 2017; Rettinger \& Andrews, 2010) — that is, research guided by theories of offending that have historically been risk-focused. Within the correctional literature, developmental criminologists, sociologists, and psychologists, as well as correctional researchers, have typically maintained that general theories of criminal behaviour adequately explain criminal conduct, regardless of one's gender ${ }^{1}$. This traditional correctional research is considered to be gender-neutral — that is, it uses a "one size fits all" approach. In contrast, approaches that are geared towards addressing the unique needs of girls and women are referred to as gender-responsive.

Arguably the most influential evidence-based gender-neutral perspective on effective offender assessment and treatment has been Bonta and Andrews' (2017) risk, need, and responsivity (RNR) model. This model highlights: 1) the risk principle, which emphasizes the importance of utilizing treatment intensity that is proportional to offenders' risk level, 2) the need principle, which states that treatment should target dynamic criminogenic needs, and 3) the responsivity principle, which is split into two

\footnotetext{
${ }^{1}$ For the purpose of this study the term gender will be used in a binary sense to refer to cis men and cis women. The term cis is used to denote anyone whose gender identity matches the sex they were labelled at birth. Incorporating a non-binary definition of gender was beyond the scope of the current study. This is a limitation of the current study and future research is encouraged to examine the role of gender in a nonbinary sense (including those who identify as bigendered, demi-gendered, nongendered, other-gendered, and genderfluid) with respect to changes in dynamic risk and strength factors and recidivism.
} 
main components: general and specific responsivity. General responsivity states that treatment should be grounded in cognitive social learning methods, where as specific responsivity states that these methods should be tailored to the unique characteristics (e.g., gender, race), and learning styles of the individual. Importantly, in the latest version of the RNR model, the role of strengths has been highlighted in three of the fifteen overarching principles. Specifically, RNR researchers have acknowledged the importance of routinely assessing strengths, in conjunction with assessing criminogenic needs, in order to better inform case management and treatment planning. Strengths have also been referenced in conjunction with specific responsivity concerns; that is, individual personal, interpersonal and circumstantial strengths should be considered when case management planning. For instance, some important strengths to consider include problem-solving skills, prosocial peers, family values, and successful employment. Finally, treatment services are encouraged to reduce major dynamic risk factors as well as enhance strength factors by promoting the overcoming of barriers and strength-building (Bonta \& Andrews, 2017).

Given that individuals involved in the criminal justice system are predominately male, research has typically focused on males and has underscored factors found to be predictive of male offending. This body of research has identified the following Central Eight risk factors as having the strongest empirical support: criminal history, antisocial attitudes, antisocial peers, antisocial personality, family/marital dysfunctions, educational/employment deficits, substance use, and inappropriate use of leisure time (Bonta \& Andrews, 2017). There is an abundance of support for these gender-neutral risk factors among males and also females (Andrews et al., 2012; Olver, Stockdale, \& 
Wormith, 2014; Scanlan, Yesberg, Fortune, \& Polaschek, 2020). However, there has been some contrary evidence (e.g., Van Voorhis, Wright, Salisbury, \& Bauman, 2010) whereby the inclusion of additional gender-responsive risk factors such as low selfesteem and trauma were found to improve predictive accuracy for justice-involved women over and above the Central Eight risk factors.

As previously mentioned, gender-responsive factors are factors hypothesized to be especially important for females. Within the literature, a myriad of terms have been used interchangeably with gender-responsivity, including gender-salient, gender-specific, and gender-informed. As such, within the current study, while the term genderresponsive denotes a focus on girls and women, the term gender-informed will be used to describe an approach that incorporates multiple theoretical perspectives to consider the needs of both males and females in order to understand the similarities and differences between genders (Blanchette \& Brown, 2006).

Despite the overwhelming empirical support for the mainstream correctional perspective, a subset of researchers (both quantitative and qualitative) have come together in what is known as the feminist pathways perspective (or more recently, pathways theory), a gender-responsive perspective to offender behaviour (e.g., Belknap, 2015; Chesney-Lind, 1997; Van Voorhis, 2012). This perspective posits that traditional correctional theories are androcentric in nature and fail to consider the distinct needs of justice-involved females. Pathways researchers argue that because justice-involved females experience victimization, internalizing mental health problems, economic marginalization, and substance abuse at increased rates in comparison to justice-involved males (e.g., Belknap, 2015; Chesney-Lind \& Sheldon, 2003; Chesney-Lind \& Pasko, 
2013), these variables are important risk factors that should be prioritized among justiceinvolved females during the assessment and treatment process. Importantly, while there is some overlap in risk/need factors identified by both pathways theory and RNR researchers, such as substance abuse, most needs identified by pathways researchers are not considered to be significant in and of themselves by gender-neutral theorists. Generally, these factors have been considered specific responsivity factors, such as mental health and trauma, and are only thought to influence criminal behaviour through the Central Eight risk factors (Andrews \& Bonta, 2010).

In addition to the gender-neutral and gender-responsive perspectives, a strengthsbased perspective of offender rehabilitation has emerged in the literature over the past 15 years - the Good Lives Model (GLM; Ward, 2002; Ward \& Stewart, 2003). This theoretical model posits that to promote successful rehabilitation, treatment efforts should focus on developing offender skills, values, and attitudes that are positive and prosocial, and provide offenders with positive resources (Barnao, Robertson, \& Ward, 2010). Ward, Mann, and Gannon (2007) have suggested that the GLM is beneficial in that it encompasses a holistic, wider lens to understanding offending behaviour and can assist case management planning. Importantly, this theoretical perspective has been criticized for its lack of empirical support and failure to consider the importance of offender risk (Bonta \& Andrews, 2003).

Although gender-neutral researchers typically focus on risk/need factors, they recognize that strengths can assist with case management planning, treatment services, and highlight the importance of incorporating both risk factors and strengths in assessment protocols (Bonta \& Andrews, 2017). Pathways theorists also value the 
importance of strengths factors for women; however, pathways theorists have nonetheless conducted predominately risk/need-based research rather than strengths-based research. Ultimately, while there is some overlap among the gender-neutral, gender-responsive, and strengths-based perspectives, further integration of these theories can have positive implications on many aspects in the corrections field. Specifically, further integration may bring the field closer towards a more holistic approach to offender assessment and rehabilitation; assessing all areas of an offender's life, while also considering the unique differences between offenders. Arguably, by capturing all relevant risk and strength factors in assessment, a more accurate calculation of risk will emerge. Further, theoretical integration can lead to more comprehensive rehabilitation efforts by which treatment programs may accurately target all relevant gender-neutral and gender-responsive risk/need factors, as well as help identify and build strengths in an offender's life, to promote success.

The overarching goal of the dissertation is to examine if and how, strengths aid in risk assessment and whether strengths operate differentially as a function of gender using a person-centered approach and growth curve modeling. Study 1 employs a genderinformed risk assessment tool, the Service Planning Instrument (SPIn; Orbis Partners, 2003), to assess how and whether total risk and strength scores change over time, and whether this change varies as a function of gender. The second part of this study will examine whether the rate of change in total strength and risk scores predict recidivism. Study 2 also uses the SPIn to examine how specific needs and strengths from the genderneutral and gender-responsive camps co-occur to create theoretically-integrated typologies of offenders. These typologies will also be assessed to determine if they are 
similar for men and women, and if they remain stable or change over time. Finally, the second part of this study will examine how these typologies relate to recidivism, for men and women independently.

This dissertation is organized as follows: Chapter 2 provides a detailed literature review that contextualizes both Studies 1 and 2 in terms of the guiding theoretical models and research. Chapter 3 is a stand-alone chapter for Study 1, and includes an introduction, methods, results and discussion. Chapter 4 is also a stand-alone chapter for Study 2, and includes an introduction, methods, results, and discussion. Finally, Chapter 5 is a general discussion summarizing the findings and implications from both Study 1 and Study 2. 


\section{Chapter 2: Detailed Literature Review - Theoretical Foundation}

This chapter details the theoretical frameworks informing the current dissertation.

First, the general personality and cognitive social learning theory (GPCSL), and the risk, need, responsivity (RNR) model are presented. This is followed by a summary of Pathways theory, and a review of the strengths-based literature including resilience, positive psychology, and the good lives model (GLM). Finally, the importance of integrating theoretical perspectives is discussed, setting the context for Studies 1 and 2.

\section{General Personality and Cognitive Social Learning Theory}

Correctional research has long maintained that offending behaviour can be sufficiently explained by general theories of crime, regardless of gender or ethnicity (e.g., Bonta \& Andrews, 2017). One general theory of criminal behaviour is the general personality and cognitive social learning theory (GPCSL; Bonta \& Andrews, 2017). The GPCSL posits that there are various ways individuals become involved in criminal behaviour, highlighting that individuals commit crime because of personality predispositions and his or her social learning environment (Bonta \& Andrews, 2017; Bonta, Blais, \& Wilson, 2013). GPCSL denotes that criminal behaviour occurs when the costs and rewards for committing the crime outweighs the benefits for engaging in prosocial behaviour. These costs and rewards come from a host of different areas: other people (e.g., peers, family, co-workers), internally (e.g., feelings of pride) or from the behaviour itself (e.g., adrenaline, excitement). More specifically, GPCSL can be split into

three components: general personality, cognitive, and social learning. General personality refers directly to an antisocial personality pattern, which captures rule violation and certain personality patterns related to increased criminal behaviour. The cognitive aspect 
refers to self-regulation that is both automatic and self-conscious - attitudes, values, and beliefs (Bonta \& Andrews, 2017). Finally, social learning refers to the costs and rewards for committing criminal behaviour within social contexts, such as friends and work. Overall, this theory of criminal behaviour outlines the importance of examining the interpersonal, personal, and community factors that may lead to criminal engagement, and importantly, has brought about the "what works" movement which emphasizes the importance of risk, need, and responsivity principles for correctional treatment.

Risk, need, and responsivity. While the GPCSL is a theoretical model used to explain offending behaviour, it offers practical implications. Subsumed within the GPCSL is the risk, need, and responsivity (RNR) model of offender rehabilitation, which is used to inform correctional treatment and rehabilitation efforts (Andrews, Bonta, \& Hoge, 1990). The RNR model, while originally made up of three core principles — risk, need, and responsivity — more recent versions have expanded to a 15-principle model. These 15 principles are broken down into: overarching principles, core RNR and key clinical principles, and organizational principles involving settings, staffing, and management. Each will be discussed in turn.

Three of the 15 principles are considered overarching principles. These principles highlight the following key messages: respect for the person-including delivering services that demonstrate respect for the person; the importance of utilizing psychological programming based on empirical psychological theory; and the importance of enhancing crime prevention services to extend services outside of corrections such as health and social services. 
The next group make up the core principles and key clinical issues. These nine principles emphasize the use of human services such as social and clinical services required to address crime. The risk principle, as previously mentioned, states that treatment should be proportional to the offenders' risk level. The need principle indicates that treatment should target dynamic risk factors, or criminogenic needs. Specifically, the Central Eight represent the most influential factors that impact the decision to engage in criminal behaviour. Of these risk factors, criminal history is the only static, unchangeable risk factor, whereas the remaining seven are dynamic — that is, they can change, either positively or negatively (Bonta et al., 2013). These dynamic factors include antisocial attitudes, antisocial peers, antisocial personality, substance use, family/marital, employment/education, and leisure activities. The assessment of dynamic risk factors is recommended as they permit the assessment and monitoring of offender change in risk level and can help guide treatment targets and rehabilitation efforts (Bonta \& Andrews, 2017). The responsivity principle is split into two: general responsivity, which emphasizes that treatment should incorporate cognitive social learning methods, and specific responsivity, which denotes that treatment should be tailored to the unique learning styles and characteristics of the individual, such as gender and race. These core principles and key clinical issues also emphasize the importance of targeting multiple dynamic risk factors, particularly among high risk cases, as well as highlight the role of strengths that can be used in the context of specific responsivity and can assist with the prediction of reoffending. Finally, there is a need for structured assessment as opposed to unstructured professional judgement, as well as utilizing professional discretion, whereby in some instances the use of overrides may be warranted. 
Finally, the third set of principles are used in the organizational context and highlights the importance of having support for RNR in policy and upper management. Specifically, RNR should be embedded within community-based services, which should be delivered by staff with high-quality relationship skills and structuring skills to enhance effectiveness. Finally, management should promote RNR within the selection, training, and supervision of staff. Incorporating these principles can enhance rehabilitation and supervision efforts leading to a decrease in offending outcomes.

RNR: Empirical support and critiques. There is a considerable amount of metaanalytic reviews that have been conducted over the past 30 years supporting the GPCSL theory and RNR principles. Specifically, an abundance of meta-analyses have validated the predictive accuracy of the Central Eight risk factors in predicting general and violent recidivism (Andrews et al., 2012; Andrews, Bonta, \& Wormith, 2004; Dowden \& Andrews, 2000; Dowden \& Brown, 2002; Gendreau, Little, \& Goggin, 1996; Lowenkamp, Holsinger, \& Latessa, 2001; Olver et al., 2014; Rettinger \& Andrews, 2010; Simourd \& Andrews, 1994), and have found support for the treatment and assessment of these factors across a wide range of offender populations, including youth (e.g., Cottle, Lee, \& Heilbrun, 2001) and mentally disordered offenders (e.g., Bonta, Law, \& Hanson, 1998). While there is a vast amount of support for the RNR principles and Central Eight risk factors focusing on boys and men, there is also considerable research focusing on girls and women. To date, eleven meta-analyses have been conducted specifically examining women and girls, and the predictive validity of the Central Eight risk factors assessed on the Level of Service Tools (Bonta \& Andrews, 2017). Several of these metaanalyses assessed youth studies (e.g., Green \& Campbell, 2006; Hubbard \& Pratt, 2002; 
Olver, Stockdale, \& Wormith, 2009; Pusch \& Holtfreter, 2018; Schalbe, 2008; Simourd \& Andrews, 1994; Smith, Cullen, \& Latessa, 2009). In contrast, there were two metaanalyses conducted on adult samples (e.g., Olver et al., 2014; Smith et al., 2009), and one meta-analysis conducted by Andrews et al. (2012) included samples of both youth and adults. Collectively, the results of these meta-analyses have highlighted the utility of the Central Eight risk factors with samples of girls and women. The most recent metaanalyses are described in detail.

Andrews et al. (2012) included samples of both youth and adults across five intrastudy datasets, all of which contained both genders to comprise a total of 2,069 males and 354 females, and follow-up times ranged from 1 year up to 4.2 years. Overall, it was found that all Central Eight were predictive of criminal recidivism for both males and females. In fact, each of the domains was consistently predictive for both females (AUCs ${ }^{2}$ ranging from .63 to .75) and males (AUCs ranging from .61 to .71).

However, only the criminal history and the substance use domain were significantly more predictive for the females than the males $(t=3.06, p=.04$ for criminal history and $t=$ $3.78, p=.02$ for substance use).

The most recent meta-analysis conducted on studies incorporating samples of justice-involved girls and boys was Pusch and Holtfreter (2018). This study conducted separate meta-analyses for 5,966 girls (30 effect sizes) and 23,308 boys (39 effect sizes). Results found that the Youth Level of Service/Case Management Inventory (YLS/CMI; Hoge \& Andrews, 2003) significantly predicted general recidivism for both boys $(r=.28$,

\footnotetext{
${ }^{2}$ An area under the curve (AUC) of .50 indicates a prediction that is equal to chance, while an AUC of 1.00 indicates perfect accuracy. Within the field of correctional psychology, AUC values ranging from .56 to .63 indicate small effect sizes, AUCs between .64 and .70 indicate medium effect sizes, and AUCs exceeding .70 indicate large effect sizes (Rice \& Harris, 2005).
} 
$95 \% \mathrm{CI}[.25, .31])$ and girls $(r=.25,95 \% \mathrm{CI}[.21, .29])$. To assess violent recidivism, a subsample of 745 girls ( 8 effect sizes) and 7,715 boys (20 effect sizes) were used. Results indicated that the YLS/CMI significantly predicted violent recidivism for both boys $(r=$ $.30,95 \%$ CI $[.25, .34])$ and for girls $(r=.25,95 \%$ CI $[.21, .30])$, suggesting that the tool did not predict recidivism outcomes better for one gender over the other.

Finally, the most recent meta-analysis focusing on adult samples of justiceinvolved men and women and the predictive validity of the Level of Service tools was Olver and colleagues (2014). Using a sample of 137,931 justice-involved adults $(80.5 \%$ men) from 128 studies (151 unique samples), the ability of the domain scores to predict general and violent recidivism for both genders independently, were assessed. Results indicated that the Central Eight domains predicted general recidivism for both women $\left(r_{\text {random }}=.13\right.$ to $.30 ; r_{\text {fixed }}=.12$ to .37$)$ and men $\left(r_{\text {random }}=.12\right.$ to $.30 ; r_{\text {fixed }}=.06$ to .34$)$. Similarly, for violent recidivism, all domains were significant predictors for both women $\left(r_{\text {random }}=.10\right.$ to $.23 ; r_{\text {fixed }}=.10$ to .23$)$ and men $\left(r_{\text {random }}=.12\right.$ to $.24 ; r_{\text {fixed }}=.12$ to .29$)$. For women the substance use and personal/emotional domains had significantly larger effect size magnitudes than men in the prediction of general recidivism. Taken together, the results of these three meta-analyses highlight the gender-neutrality of the Central Eight domains whereby these domains significantly predict general and violent reoffending for both males and females, which denotes the utility of the tool with justice-involved women.

Although there has been an abundance of support for this model (e.g., Andrews et al., 2012; Bonta \& Andrews, 2017), it has been criticized for its lack of motivation goals and for failing to recognize the importance of personal identity and non-criminogenic 
needs (Laws \& Ward, 2011; Ward et al., 2007; Ward \& Stewart, 2003). While originally the focus of traditional mainstream correctional research was on risk/need factors and the assessment of risk level, within the past 30 years, more emphasis has been given towards the incorporation of strengths in risk assessment and case management protocols. For some RNR theorists, the absence of criminogenic needs is considered to be a strength (Polaschek, 2012). However, strengths are also discussed within the context of the responsivity principle, indicating that treatment efforts should match and be mindful of offenders' motivations, readiness for change, strengths, and values (Bonta \& Andrews, 2003; 2017). Although traditional assessment tools, such as the Level of Service/Case Management Inventory (LS/CMI; Andrews et al., 2004), allows for the identification of strengths for each of the eight risk/need areas (Andrews et al., 2004), they are not part of the quantitative scoring of the LS/CMI for risk level (Blanchette \& Brown, 2006). Nonetheless, RNR has guided treatment and risk assessment protocols around the world, and has been considered the gold standard for effective treatment-based rehabilitation efforts.

\section{Gender-Responsive Pathways Theory}

Pathways theory (PT) is an alternate explanation of female crime; comprised of empirical work produced by scholars including psychologists (Salisbury \& Van Voorhis, 2009), criminologists (Covington \& Bloom, 2006), and sociologists (Richie, 1996), stemming over the last 35 years. Unlike GPCSL, the defining tenet of the PT is that gender matters - the starting assumption is that females enter into crime through unique pathways from their male counterparts. Pathways in the context of PT does not refer to a causal sequence of events, but rather refers to the relationship among various risk factors 
and the sequence of events that may have led women and girls to become involved in the criminal justice system. The underlying notion is that women and girls enter into crime because of negative life events which include childhood adversity, economic marginalization, and dysfunctional relationships (Belknap, 2015; Chesney-Lind, 1997; Pasko, 2008; Simkins \& Katz, 2002). Repeat exposure to these negative life events lead to negative coping strategies which include running away from home or using drugs and alcohol. From here, these negative coping patterns can lead to survivalist crimes including prostitution, dealing drugs, or robbery (Blanchette \& Brown, 2006; ChesneyLind, 1997). One important takeaway is that PT continuously finds childhood trauma (e.g., physical, sexual, emotional abuse, and neglect) to be a key feature in the lived experienced of justice-involved women and girls (Bloom, Owen, \& Covington, 2003; Pasko, 2008). As such, pathways researchers argue that traditional theories of criminal behaviour are insufficient in explaining female offending behaviour due to their androcentric nature (Belknap, 2015; Blanchette \& Brown, 2006; Chesney-Lind, 1989; Chesney-Lind \& Pasko, 2013; Covington \& Bloom, 2006). In particular, pathways researchers argue that females are criminalized for their survival strategies and that traditional theories of criminal offending have typically ignored the importance of trauma and victimization on female offending (Belknap, 2015; Chesney-Lind \& Pasko, 2013).

Since the 1990s, understanding of justice-involved girls and women has increased substantially with significant empirical research assessing women's pathways into offending, women's specific risk and need factors, and treatment and rehabilitation approaches that best address the needs of women (Van Voorhis, 2012). Genderresponsive researchers have also highlighted the importance of focusing on strengths with 
justice-involved females in terms of strengthening positive relationships, increasing wellbeing, and empowering women (Hannah-Moffat, 2009; Van Voorhis, 2012). Blanchette and Brown (2006) have suggested that incorporating a strengths-based approach is beneficial, especially with justice-involved females since most do not pose a significant risk to reoffend. Considerable advancement in the work of gender-responsive scholars have led to a number of gender-responsive programs and policies developed to account for the unique needs of justice-involved women and girls and incorporate a strengthsbased approach (Covington, 2016, 2018; Wattaporn \& Holtfreter, 2014). For instance, an influential policy document outlining five key principles of gender-responsive practice was created by Bloom et al. (2003) under the National Institute of Corrections. These five key principles include: (1) recognition that there are a range of differences between justice-involved men and women, including social, environmental, and criminogenic factors; (2) safety, respect, and dignity must be key components of corrections; (3) programs and services should emphasize the importance of healthy relationships with children, family, intimate partners and community; (4) through services and programs, substance use, trauma, and mental health issues should be targeted; and (5) through services and programs, opportunities to improve the socio-economic status of women should be provided. Notably, these principles have been used to inform several genderresponsive programs for women (and more recently, men) including Covington's (2013, 2016) programs, Helping Women Recover (2019) which incorporates cognitive behavioural approaches, mindfulness, healthy relationships, sexuality, self-exploration, and spirituality, and Beyond Violence (2016) which incorporates cognitive behavioural approaches, mindfulness, coping skills, as well as works with the violence that the 
women experienced. These programs incorporate gender-neutral techniques (e.g., cognitive behavioural techniques), gender-responsive experiences (e.g., trauma, poor coping skills), and emphasizes strengths (e.g., healthy relationships, spirituality).

Pathways theory: Empirical support and critiques. Pathways scholars have aimed to address the limitations of traditional theories through the use of both qualitative and, more recently within the past 10 years, quantitative research. Qualitative research, which stems from descriptions of characteristics and scenarios, allows participants to explain their situations and tell their stories in their own voices using retrospective, indepth, face-to-face interviews and file reviews (Belknap, 2015). Results from this body of work have highlighted several key emerging themes that may arguably increase the risk of females' involvement in the criminal justice system — namely these include trauma and victimization, poverty and economic marginalization, mental health issues, poorparenting and dysfunctional relationships, and reduced self-efficacy and self-esteem (Chesney-Lind, 1997; Chesney-Lind \& Pasko, 2013; Daly, 1992; Dehart, 2008; Finkelhor \& Baron, 1986; Simkins \& Katz, 2002).

One of the most influential qualitative studies has been Daly's (1992) study which was one of the first to denote the different pathways that women tend to follow to the criminal justice system; that is, the different typologies of justice-involved women. Upon qualitatively examining the pre-sentence reports of 40 justice-involved women, Daly (1992) determined that five pathways emerged. The first are street women, which refer to women who run away from home to escape victimization, use substances as a coping mechanism, drop out of school, and rely on prostitution for money. The second are harmed and harming women, which refer to women who suffered abuse and neglect as 
children resulting in emotional and psychological issues and chaotic home lives, and used substances to cope. The third, are drug-connected women, who had limited criminal history, but experimented with and sold drugs with either a male partner or family member. The fourth are battered women, who were physically abused by a romantic partner, and the fifth typology of women are other/economically motivated women, who committed crime because of financial motivation. Overall, Daly's (1992) research highlights a number of themes that justice-involved women have in common that make up multiple pathways, including victimization, substance use, mental health concerns, economic marginalization, and involvement with antisocial romantic partners.

Since Daly, an extensive body of qualitative research contextualizing the pathways women take into crime has followed (e.g., Chesney-Lind, 1997; Dehart, 2008; Gilfus, 1992; Richie, 1996). More recently, typological research using quantitative methods has emerged (see Chapter 4, Study 2, which focuses on the typology literature in greater detail). While the majority of earlier research informing PT is qualitative in nature, quantitative research (cross-sectional research and, most recently, prospective and longitudinal research) has emerged and is now prominent among PT scholars. For instance, many descriptive cross-sectional and prevalence studies have demonstrated empirical gender-differences in pathways-informed variables with larger samples of females using inferential statistics. There is a considerable amount of research highlighting the gender differences in prevalence of gender-specific variables including victimization, internalizing mental health issues, economic marginalization, substance use, and poor family relationships (Belknap \& Holsinger, 2006; Blanchette \& Brown, 2006; Bloom et al., 2003; Salisbury \& Van Voorhis, 2009). 
One of the few studies to incorporate a male comparison group was a study conducted by Jones, Brown, Wanamaker, and Greiner (2014). Using a large sample of justice-involved boys $(n=1,175)$ and girls $(n=663)$, the study found that girls had many gender-responsive needs, including experienced abuse $(28.2 \%)$, diagnosed mental health problems $(8.4 \%)$, and living in poverty $(25.6 \%)$. However, it was also found that some girls had a variety of traditional, gender-neutral risk factors, including antisocial peers (70.7\%) and substance abuse (45.6\%). Overall, there was an equal distribution which emerged between girls who belonged to a gender-responsive pathway to crime $(47.8 \%)$ versus girls who belonged to the more traditional, antisocial pathway to crime $(51.7 \%)$. In contrast, just under a quarter $(24.6 \%)$ of boys reported a combination of genderresponsive and gender-neutral risk factors (i.e., runaway attempts, child abuse, and mental health). The results of this study highlight the importance of considering genderresponsive theories of criminal behaviour.

Although prevalence is important to examine, it does not decipher whether these variables predict offending behaviour. Nonetheless, these prevalence studies have demonstrated the importance of gender-responsive variables in the lives of justiceinvolved females, and even justice-involved males (e.g., Jones et al., 2014), and further lend support for the inclusion of these variables in risk assessment protocols. As such, it is imperative to use prospective and longitudinal research designs to examine whether these variables are predictive of reoffending.

One influential study that has examined the predictive validity of genderresponsive risk factors was Van Voorhis et al. (2010). This study included both genderneutral (Central Eight) risk factors, as well as hypothesized, gender-responsive risk 
factors, including victimization and abuse, relationship problems, mental health, selfefficacy, and parental issues. Three prison samples from Colorado, Missouri, and Minnesota, three probation samples from Maui, Missouri, and Minnesota, and two prerelease samples from Colorado and Missouri was used to examine relevant risk factors for females. These samples were comprised entirely of women offenders and sample sizes ranged from 134 to 313 women offenders in each subsample.

Not surprising, it was found that gender-neutral risk factors were predictive of recidivism at both 1- and 2-year follow-up times across samples on probation (AUCs of .71 for Minnesota, .72 for Maui, and .69 for Missouri). When optimal gender-responsive predictors were added (these included self-efficacy and parental issues for all probation samples as well as additional gender-responsive predictors that varied between samplesSee Van Voorhis et al., 2010 for further details), the predictive ability increased (AUCs of .74 for Minnesota and Missouri), with the exception of the Maui sample. For the prison samples, only the AUC for the Minnesota sample was found to increase with the inclusion of gender-responsive predictors (AUC increased from .68 to .70); the predictive ability did not increase with the addition of the gender-responsive variables for Colorado (AUC remained .58) or Missouri (AUC remained .64). Finally, for the pre-release samples, existing gender-neutral risk factors were weakly predictive of technical violations and rearrests ( $\mathrm{AUC}=.60$ and .55 , respectively), for both subsamples. The addition of hypothesized gender-responsive risk factors did not add incremental predictive validity for the Colorado sample. However, for the Missouri sample, the gender-responsive risk factors added predictive validity for technical violations (AUC went from .58 to .62) and for incarceration (AUC increased from .66 to .70). 
While this study demonstrates the importance of considering alternative, genderresponsive risk factors in addition to gender-neutral risk factors, more research is needed to examine how these variables influence criminal recidivism across larger samples and among men. Further conceptualization of the needs highlighted by gender responsive advocates is needed to determine how these factors are accounting for criminal behaviour for females and even males (Hannah-Moffat, 2009). Overall, many scholars have criticized the PT for being too one-dimensional nature (Blanchette \& Brown, 2006; Odgers, Moretti, Burnette, Chauhan, Waite, \& Reppucci, 2007). That is, PT explains female criminal behaviour solely through survival-based mechanism, failing to consider other means of female criminality (Kruttschnitt, 2016). Additionally, while researchers have attempted to incorporate strengths in the treatment of justice-involved females, ultimately further research is needed to assess the incorporation of strengths in risk assessment models for women (Blanchette \& Brown, 2006). Finally, limited PT research has incorporated multi-wave longitudinal research designs and no research has examined how gender-responsive factors may change over time.

\section{Strengths-Based Correctional Perspective}

In the past 15 years, theories focusing on strengths, simply defined as positive internal and external factors in an individuals' life, have emerged (e.g., Good Lives Model [GLM]; Ward, 2002). With guidance from strengths-based theories, researchers and practitioners have begun to incorporate strengths into risk assessment protocols and correctional treatment approaches. Three strengths-based theories that have greatly influenced the field of corrections include: resilience literature, positive psychology, and Good Lives Model. However, prior to describing these theories, it is imperative to review 
the various definitions in the literature and how they are used, both interchangeably and differentially.

\section{Definitional Caveats-Strengths, Promotive, and Protective Factors}

Despite the growing interest in strengths and the incorporation of offender strengths within risk assessment, there have been major inconsistencies with the way strengths are defined and conceptualized. Some scholars have suggested that a strength is merely the absence of a risk factor, proposing that a variable cannot be viewed as both a risk and a strength (Ogloff \& Davies, 2004). However, there is evidence to suggest that strengths can occur simultaneously with risk factors and can account for the variability in specific outcomes (Jones, Brown, Robinson, \& Frey, 2015, 2016; Laub, Nagin, \& Sampson, 1998; Sampson \& Laub, 2005). For example, one study conducted by Jones et al. (2015) used data from the Service Planning Instrument (SPIn; Orbis Partners, 2003) Pre-Screen scores collected on 3,656 men and women on community supervision in Canada. Using a median split, Jones et al. (2015) compared lower risk cases to higher risk cases in terms of strengths and their relationship to reoffending. Results indicated that there was a buffering effect among the higher risk group where $23.2 \%$ of the higher risk cases with high strengths (determined using a median split) reoffended over an 18-month follow-up, in comparison to $36.4 \%$ of higher risk cases with low strengths. Upon looking at the lower risk cases, a buffering effect was also observed whereby those with higher strengths reoffended at a rate of $6.1 \%$, in comparison to $12.5 \%$ of lower risk cases with low strengths. These results demonstrate that strengths can assist with explaining the variability between offenders among various risk classification levels in terms of reoffending rates. 
One way that the term strength has been used is as an umbrella term to describe a positive aspect of an individual's life (Jones et al., 2015). Other researchers further contend that a strength must add something positive to an individual's life (Lodewijks, de Ruiter, \& Doreleijers, 2010). This suggests that the mere absence of a risk would not necessarily imply a strength — for instance, the absence of procriminal peers is not in itself a strength; but having prosocial peers would be a strength. The absence of both prosocial and antisocial peers are considered neutral — that is an absence of risk and strength. The term strength is generally referred to as positive internal resources, such as an individual's priorities, goals, and values, and/or external resources, such as prosocial peers, and supportive family that an individual has available to them (Laws \& Ward, 2011). Under this umbrella term, strength, we often see two other terms used within the criminal justice literature-protective and promotive.

Unfortunately, the terms promotive and protective are less clearly defined. Within the correctional literature, arguably, the most common definition of a promotive factor is a variable that is negatively correlated with recidivism (i.e., a factor that leads to a positive outcome) regardless of one's overall level of risk (Farrington, 2003; Farrington, Ttofi, \& Piquero, 2016). In contrast, the term protective is thought to capture the concept of resilience - that is, a protective effect occurs when a positive variable significantly buffers criminal outcome among those who are high risk (i.e., leads to a more positive outcome), whereas for those deemed to be low risk, the positive variable has a minimal effect (Farrington et al., 2016; Sampson \& Laub, 2005). A recent study, however, conducted by Brown, Wanamaker, Wagstaff, and Robinson (2020) posit that even if strengths demonstrate an effect on those deemed low-risk, it should still be considered a 
protective (or buffering) effect. The rationale behind this argument being that the origins of buffering definitions emerged from the resiliency literature among true at-risk samples exposed to adversity, whereas corrections populations have already surpassed the at-risk designation as they have already entered the justice system.

Although there are various other definitions of promotive, protective, and strength factors (e.g., Glowacz \& Born, 2015; Ward, 2017), for the purpose of this study, the definitions described above will be used. Because terminology is one of the most challenging issues in the strengths-based literature, it is important for measures, such as risk assessment tools, to use the proper terms to describe their items. Overall, Jones et al. $(2015,2016)$ have suggested that incorporating a strengths-based assessment approach leads to four positive outcomes: (1) encourages clients to engage in case planning, (2) allows practitioners to build stronger rapport with clients, (3) enhances client motivation and engagement in the rehabilitation process, and (4) assists with identification of positive resources and prosocial supports the client has in their life. As such, the current research will help distinguish the impact that strengths play on the criminal justice population — for both males and females — in terms of risk assessment, successful offender outcome, and potential treatment effort.

Resilience literature. Resilience research dates back to 1936 when Healy and Bronner questioned why some people engage in "bad" behaviour and others do not. This concept was re-visited in 1974, when Anthony referred to the phenomena of invulnerable children, specifically commenting on the large number of youths that appear to thrive despite their engagement in high risk situations including exposure to antisocial models and environmental stressors. The term resilience was first adopted in the developmental 
psychology literature in the late 1970s in the context of psychopathology and child development (Rutter, 1979). At this time, researchers were interested in children's longterm responses to environmental and familial stressors. Given exposure to similarly high risk, negative environments, some youth developed psychopathologies and maladaptive coping responses (including delinquency) while others displayed resilience even after facing adverse situations and grew up to be prosocial members of society (Garmezy, 1973; Rutter, 1979; Werner \& Smith, 1977).

Importantly, Werner's research $(1989,2005)$ was fundamental in establishing that resilient youth are characterized by various strengths, which in turn reduce their likelihood of experiencing negative outcomes. Werner and Smith (1982) first introduced the term protective factor in the context of studying resilient children; they defined a protective factor as a positive factor that has a positive effect on adaptation among highrisk children.

In an influential longitudinal study updated in 2005, Werner demonstrated that protective factors buffer the impact of adverse childhood situations on developmental outcomes. A total of 698 participants were followed from the time of their birth in 1955 until 2005, resulting in a 40-year follow-up period. Approximately $30 \%$ of the sample was classified as high risk, having experienced multiple risk factors in childhood including pre- and/or perinatal complications, parental psychopathology, high levels of family conflict, and poverty. Of these at-risk children, approximately two-thirds experienced negative outcomes by adolescence, including mental health issues, learning and behavioural difficulties, and delinquency. Notably, the remaining one-third of at-risk children were resilient and achieved all developmental milestones — such as completing 
their education, holding down steady employment, maintaining a stable family structure in adulthood, and having no reported conflicts with the law. In fact, the achievements attained through education and employment among these resilient children either met or surpassed the accomplishments of their low risk counterparts - that is, those from financially privileged backgrounds and stable homes.

Werner (2005) identified various protective factors at the individual, family, and community level that differentiate resilient high-risk cases from high risk cases that experienced negative outcomes. Notably, resilient individuals were characterized by higher levels of self-efficacy, strong problem-solving skills, were more relational in nature-including having strong emotional connections with one or more prosocial caregiver, such as a grandparent or aunt/uncle, and had a prosocial support network within the community (e.g., involvement in a church group). Werner's study clearly exemplifies the co-existence of risk and protective factors within a given individual, and the role that protective factors can play in reducing the impact of risk.

Since Werner's (2005) study, resilience research has focused on identifying various factors that are most predictive of resilience in different contexts of adversity (Fox, 2019; Lösel \& Farrington, 2012; Vanderbilt-Adriance \& Shaw, 2008). Specifically, in most contexts, resilience factors (often referred to as protective factors or strengths in the resilience literature) can come from different areas: the community, family, friends, school, and within the individual. To summarize, research has suggested that personal (e.g., self-esteem/self-concept), inter-personal (e.g., family cohesion) and community (e.g., neighborhood safety) strengths are the underlying factors guiding resilient outcomes (Masten, 2013). However, the cumulative effect of these factors is most 
predictive of resilience (Glowacz \& Born, 2015), although further research is required. More research is needed on specific types of adversity (e.g., experiencing various childhood traumas), and how specific strengths influence the likelihood of refraining from criminal behaviour.

Positive psychology. Positive psychology, which dovetails with the study of strengths and resilience, is a recently recognized sub-discipline of psychology after it was discussed in Martin Seligman's Presidential Address at the American Psychological Association conference in 1998 (see Fowler, Seligman, \& Koocher, 1999; Seligman, 2000). Positive psychology is defined as the study of positive emotions, character traits, and institutions that permit people to grow (Gable \& Haidt, 2005; Seligman, Steen, Park, \& Peterson, 2005; Sheldon \& King, 2001). Although the current, established field of positive psychology is in its infancy, it can be traced back to the early 1900s with James' (1902) work on healthy mindedness and Allport's (1961) work on pattern and growth in personality. Although positive psychology appeared long after the study of resilience and strengths (Yates \& Masten, 2004), it was only after the emergence of positive psychology that the correctional literature began to focus on offender strengths. Following its recognition as a formal discipline, researchers dedicated their attention to expanding the conceptual framework of the field. However empirical testing has only occurred in the last few years (Rao \& Donaldson, 2015). Regardless, the positive psychology framework is increasingly being applied internationally and across a wide range of contexts, informing poverty alleviation, health care, and environmental issues (Golden-Biddle \& Dutton, 2012), as well as offender rehabilitation. 
The Good Lives Model. In an effort to move towards a more strengths-based approach to offender rehabilitation, Ward and colleagues (i.e., Birgden, 2002; Laws, 2002; Ward, 2002; Ward \& Stewart, 2003) developed the Good Lives Model (GLM). The main goal of GLM is to manage offenders' risk for reoffending while promoting prosocial means to achieve goals (Ward \& Maruna, 2007; Ward \& Stewart, 2003). This approach is a proposed correctional alternative to the RNR model and is heavily grounded in positive psychology. While GLM is similar to traditional rehabilitation models in that it aims to reduce reoffending, instead of promoting the avoidance and reduction of risk, it encourages the enhancement of individual strengths and positive societal reintegration (Ward et al., 2007). Specifically, GLM posits that people offend because they are trying to obtain a certain valued goal, however, the means to obtain this goal is manifested in an antisocial way, rather than a prosocial way (Ward, 2002; Ward \& Brown, 2004). For example, to obtain happiness, an individual may believe he/she requires the attainment of financial assets. However, while most people use prosocial means to achieve financial security (e.g., employment), some people commit crime. As such, this criminal act is reflective of a maladaptive approach to obtaining the goal of finances and thus happiness for this individual (Ward \& Stewart, 2003). GLM theorists argue that intervention and programming should take place in a pro-social way-whereby the focus is on building individual strengths, rather than focusing on removing or managing a problem through restrictive means. Because offenders are thought to lack the prosocial means necessary to attain valued outcomes, GLM emphasizes the importance of helping offenders develop the skills, values, and attitudes they need as well as providing 
offenders with the resources required to attain these outcomes in prosocial, satisfying, and socially acceptable ways leading to a crime-free lifestyle (Barnao et al., 2010).

Ward and colleagues (2007) argue that a strengths-based approach brings a wider lens to understanding offending behaviour and can lead to a more holistic approach to case management planning. Specifically, Ward and colleagues (2007) argue that GLM takes a more humanistic approach and considers personal identity, as well as highlights the important role of human needs and how they are linked to offending behaviour. GLM researchers also stress the importance of developing a strong therapeutic relationship with offenders and emphasize positive goal attainment to enhance motivation to change (Ward et al., 2007). Overall, the argument suggests that GLM provides a more comprehensive and tailored approach to offender rehabilitation than more risk-focused approaches.

Strengths-based approaches: Empirical support and critiques. The literature on the utility of strengths in correctional settings has been mixed, particularly with respect to risk assessment purposes (Wanamaker, Jones, \& Brown, 2018). While several studies have underscored the importance of strength scores in predicting reoffending outcomes over and above risk scores (e.g., Lodewijks et al., 2010; Rennie \& Dolan, 2010; Shepherd, Delgado, Sivasubramaniam, \& Paradies, 2018), other studies have failed to find support for the inclusion of strengths (e.g., Dickens \& O'Shea, 2018; Dolan \& Rennie, 2008). Alternatively, other researchers have found support for the utility of strengths as responsivity factors, related to personal motivation and social support (Finseth, 2020). That is, instead of directly adding to the calculation of risk, strengths are factors that should be considered in case management and treatment should be tailored to individuals' level of strengths. Finally, as previously mentioned, there has also been 
support for a buffering effect of strengths on the relationship between risk and reoffending outcomes (Jones et al., 2015).

Aside from the mixed findings of strengths, researchers have also raised concerns around the definition and conceptualization of strengths in practice. For example, Baird (2009) and Harris and Rice (2015) argue that strengths are simply the opposite of risks, and thus are already sufficiently captured in risk assessment protocols. Similarly, Polaschek (2016) argues that in order for strength factors to truly contribute to prediction, over and above risk factors, the strength factors must represent unique variables not captured within the risk domains. Given the mixed support and the definitional and conceptual concerns around strengths - more research is needed on how strengths should optimally be considered in risk assessment protocols.

Overall, the strengths-based approaches have been highly critiqued. For example, resilience research has been criticized for not using a consistent operational definition of adversity. As such, this poses challenges for determining what specific factors predict resilience (Fletcher \& Sarkar, 2013). Positive psychology has been critiqued for not incorporating a holistic approach; the approach solely focuses on positive aspects (Snyder, Lopez, \& Pedrotti, 2011). Thus, there is a need to advance the field of positive psychology in a manner that takes a more balanced approach. Additionally, Finseth (2020) argues that the GLM focuses on the same intervention targets as RNR, but just uses a different terminology. Bonta and Andrews (2003), Andrews, Bonta, and Wormith (2011) and Looman and Abracen (2013) have also criticized GLM for its lack of empirical support. Particularly, empirical evaluations and assessments are needed to discern whether GLM enhances treatment efforts and reduces offender recidivism. 
Finally, Andrews (2012) has argued that GLM has limited practical implicationsparticularly weak assessment approaches, based on unstructured professional judgement.

\section{Chapter 2 Summary}

This chapter reviewed three theories of criminal behaviour; the general personality and cognitive social learning theory (GPCSL), the pathways theory (PT), and the good lives model (GLM), which serve as a foundation for Study 1 and Study 2. Study 1 assesses change over time in total risk and strength scores for men and women and examines whether the rate of change in total strength and risk scores predict recidivism. In contrast, Study 2 examines theoretically-integrated typologies of justice-involved men and women made up of risks, needs, and strengths from the gender-neutral and genderresponsive camps, and examines the relationship between the typologies and recidivism.

Traditional theories of criminal behaviour, such as the GPCSL, maintain that risk factors of criminal behaviour are the same for both males and females. That is, the criminogenic needs (e.g., Central Eight) of men and women are gender-neutral. Although an abundance of research has provided support for the validity of this perspective, a female-driven theory (PT), posits that because traditional theories were developed by males using predominately male samples, females are a correctional afterthought. Pathways researchers argue that theories of female criminal conduct need to be formed from the ground up and must consider females' unique pathways into crime. Pathways theorists highlight the importance of gender-responsive needs, which are often referred to as responsivity factors by GPCSL (e.g., mental health, victimization). Notably, many gender-responsive scholars disregard the use of gender-neutral risk factors with women and girls, indicating that these factors are not operationalized or captured in a way that 
reflects females' unique circumstances, despite the abundance of research supporting the utility of these factors with females. Similar to RNR theorists, gender-responsive scholars also highlight the importance of considering strengths in order to provide a more holistic approach to rehabilitation.

Given the focus on risk factors in the correctional field, strengths-based theorists and researchers (i.e., GLM researchers) have highlighted the need to examine whether risks and strengths interact to predict offender outcome. Specifically, with the development of positive psychology, correctional research has begun to shift focus to strengths. GLM is described as an alternative approach to the RNR model and instead of promoting the avoidance of risk, it encourages enhancing individuals' strengths. The theoretical model, however, remains largely empirically untested.

While pathways researchers recognize and endorse the inclusion and assessment of strengths, and RNR researchers have included strengths in their theoretical model, the focus remains on offenders' risks as opposed to their strengths. Particularly, RNR researchers, while assessing offenders' strengths in general do not use this assessment when determining an offenders' risk level to reoffend — rather, these strengths are merely used for case management purposes. Similarly, both RNR theorists and pathways researchers have limited research assessing the predictive nature of strengths with both men and women.

\section{Making the Case for Theoretical Integration}

Theoretical integration of the gender-neutral, gender-responsive, and strengthsbased approaches can lead to a holistic approach to offender assessment and rehabilitation where treatment programs may accurately target all relevant risk and need factors, as well as help identify and build strengths in an offender's life. There has been 
some empirical meta-analytic support indicating the utility of combining theoretical models, specifically for the purpose of risk assessment (e.g., Scott \& Brown, 2018) and treatment needs (e.g., Gobeil, Blanchette, \& Stewart, 2016).

One meta-analysis conducted by Scott and Brown (2018) aimed to determine whether there are specific risk and strength factors that predict recidivism differently for male and female adolescent offenders. Using 22 studies (11,952 girls; 38,649 boys) a total of 584 effect sizes were coded across predictors from both gender-neutral domains (e.g., criminal history, education and employment, attitudes, etc.) and gender-responsive domains (e.g., abuse and neglect, mental health, social history, etc.). Risk factors categorized within the gender-neutral and gender-responsive domains were found to be significant predictors of recidivism for both males and females. However, there were 12 indicators (making up 34.3\% of all indicators) that were gender-specific. This included three indicators (8.6\%) for girls: truancy at school, callous/no concern for others/low empathy, and out-of-home placements. In contrast, nine indicators $(25.7 \%)$ were genderspecific for boys: prior probation, inadequate supervision, inconsistent parenting, current school problems, delinquent influences, gang affiliation, poor use of time, physical aggression, and, antisocial attitudes. In terms of strengths, for girls, prosocial peers $(d=-0.31)$, prosocial values and attitudes $(d=-1.22)$, and rejection or absence of substance use $(d=-0.31)$ were significant of general recidivism. For boys, however, family relationships and support $(d=-0.57)$, education and employment opportunities $(d$ $=-0.68)$, prosocial peer relations $(d=-0.57)$, and rejection or absence of substance use $(d=-0.57)$ were significant effects for the males. 
Overall, this was the first meta-analysis to examine both strengths and risk/need factors among mixed-gender samples of offenders. Results demonstrated the utility of incorporating strengths in risk assessment protocols and that there were both gender differences and similarities in the predictability of various risk and strength factors. However, this research was conducted on justice-involved adolescents, and as such, more research is needed on adult samples to see if factors (both risk and strength factors) predict differentially, or equally as well, for men and women. Importantly, this research considered a comprehensive range of gender-neutral, gender-responsive, and strength factors, but more research is needed on how these factors operate in conjunction to inform risk level and offender typologies.

Another meta-analytic study conducted by Gobeil et al. (2016) examined the extent to which gender-informed treatment efforts reduced recidivism in comparison to traditional, gender-neutral treatment programs. In total, 37 studies providing 38 unique effect sizes were used ( $N=22,000$ justice-involved women). Overall, it was found that gender-informed programming improved treatment outcomes over and above genderneutral treatment programs. However, these results are dependent on the needs of women, whereby those with more gender-responsive needs rendered better outcomes when gender-informed approaches were used, where as women who had more traditional gender-neutral needs, rendered better outcomes when gender-neutral approaches were used. The gender-informed approach to treatment includes a mixture of genderresponsive and gender-neutral components (including cognitive behavioural therapy). Unfortunately, this meta-analysis did not include a comparison group of men, and did not examine the importance of strengths. Nonetheless, this research highlights the importance 
of implementing treatment interventions that are tailored to individual needs (both gender-neutral and gender-responsive), incorporating a range of treatment targets.

As such, research should assess how these three approaches (pathways, RNR, GLM) work together and operate in conjunction, as opposed to independently.

Examining how gender-neutral and gender-responsive risks and strengths influence offending behaviour, both individually and together, is required to move further towards a more holistic, integrative approach to offender assessment and particularly to enhance treatment efforts for justice-involved men and women. 


\section{Chapter 3}

\section{Study 1: Assessing Patterns of Change in Dynamic Risk and Dynamic Strength}

\section{Factors and the Relationship to Recidivism for Men and Women}

Risk factors are classified into one of two categories: static or dynamic risk factors. Static risk factors are historical factors that are unchangeable as a function of intervention (Zamble \& Quinsey, 1997). That is, static risk factors either never change, such as age at first arrest, or only change as a function of time and not intervention, such as number of prior convictions. ${ }^{3}$ These factors therefore, cannot act as treatment targets, but are useful for classifying offender's degree of risk for reoffending (Lloyd, 2015; Mann, Hanson, \& Thornton, 2010). In contrast, dynamic risk factors are changeable factors that can be targeted through correctional intervention, as they are modifiable via treatment (Andrews \& Bonta, 2010). As such, dynamic risk factors, often used interchangeably with the term criminogenic needs, measure an offender's propensity to commit an offence at a specific time, and can help inform rehabilitation efforts (Bonta \& Andrews, 2017; Simourd, 2004). Some examples of dynamic risk factors include engaging with antisocial peers, criminogenic attitudes, or poor use of leisure time.

Some researchers have further disaggregated dynamic risk factors into two categories: stable and acute dynamic risk factors (Hanson, Harris, Scott, \& Helmus, 2007). Stable dynamic factors are those that change more gradually and tend to be more long-standing traits that change over a matter of months or years. In contrast, acute dynamic risk factors are those that change more rapidly, or over shorter periods of time,

\footnotetext{
${ }^{3}$ It is important to note that although static factors are thought of as unchangeable, some static factors can change, particularly in one direction. For example, while a person's criminal history can change, this is merely as a function of time (e.g., committing additional criminal acts over time leads to increased criminal history, etc.; Bonta, 2002).
} 
such as days or weeks. Examples of stable dynamic risk factors include criminogenic attitudes or antisocial personality traits, whereas acute factors include things like drug use, which can change on a daily basis (Hanson et al., 2007).

Although there has been a substantial amount of research demonstrating the ability of dynamic risk factors to predict reoffending in both male and female samples (e.g., substance use, criminal peers, criminal attitudes; Andrews et al., 2012; Bonta \& Andrews, 2017; Wardrop, Wanamaker, \& Derkzen, 2019), the extent to which dynamic risk factors change over time, and how these changes may influence reoffending behaviour is less known, especially for women (Douglas \& Skeem, 2005).

While static and dynamic risk factors aid in determining who will recidivate and when they are most likely to recidivate, empirical findings also illustrate that strengths can influence an offender's risk to reoffend and can aid in informing rehabilitation efforts (Jones et al., 2015, 2016). In brief, strengths can be classified as protective factors if they moderate the relationship between risk and recidivism among high risk offenders, reducing the likelihood of reoffending (Jones et al., 2015, 2016; Werner \& Smith, 1992), or classified as promotive factors if they negatively correlate with recidivism regardless of one's overall risk level. Notably, strengths have been reported to be as important as risk factors in clinical practice with offenders, particularly for improving youth's engagement in services (Finseth, 2020) and for those who are mentally ill (Stübner, Groß, \& Nedopil, 2006).

Though risk assessments have continuously progressed, current practices still have many limitations. For instance, even though research has highlighted the importance of dynamic risk factors (e.g., Bonta \& Andrews, 2017), less is known about how these 
dynamic factors fluctuate over time (Lloyd, 2015). This information is essential for providing successful and timely intervention methods. Furthermore, when discussing the various categories of risk assessments, the inclusion of positive factors, or strengths, is often missing. Thus, the purpose of this study is to examine how dynamic strength and dynamic risk factors change over time and how they may change in conjunction, which is essential to further advance not only the correctional field of risk assessment, but also how we approach treatment and rehabilitative efforts.

\section{Multi-wave Assessment of Dynamic Risk}

Although many studies have employed multi-wave designs to assess dynamic risk factors (e.g., Andrews \& Robinson, 1984; Andrews \& Wormith, 1984; Bonta, 1996; Hanson \& Harris, 1998; Labrecque, Smith, Lovins, \& Latessa, 2014; Motiuk, 1999; Olver, Wong, Nicholaichuk, \& Gordon, 2007; Lewis, Olver, \& Wong, 2013; Quinsey, Coleman, Jones, \& Altrows, 1997; Schlager \& Pacheco, 2011), most of these studies have used small samples and/or a two-way design. Research has suggested that studies should include at least three timepoints to increase the accuracy of detecting change over time (Brown, St. Amand, \& Zamble, 2009). Given that the current study incorporates three or more timepoints to assess change over time, the focus of this literature review is on previous studies that have utilized at least three timepoints to assess change.

Recently, studies have been conducted examining the predictability of dynamic re-assessments using three or more timepoints among samples of youth (e.g., Clarke, Peterson-Badali, \& Skilling, 2019), forensic inpatients (e.g., Quinsey, Book, \& Skilling, 2004; Quinsey, Jones, Book, \& Barr, 2006), and sex offenders (e.g., Babchishin, 2013). Of the eight studies that have used a three-point (or more) multi-wave approach to assess 
dynamic risk scores in the general justice-involved population, the majority have focused on men (e.g., Brown et al., 2009; Jones, Brown, \& Zamble, 2010; Morgan, Kroner, Mills, Serna, \& McDonald, 2013), or have failed to disaggregate results by gender (e.g., Hanby, 2013; Howard \& Dixon, 2013; Lloyd, 2015). Only two studies have examined change in dynamic risk scores among women (e.g., Dowden, Serin, \& Blanchette, 2001; Greiner, Law, \& Brown, 2015), although Dowden et al. (2001) did not examine how changes in dynamic risk scores predicted reoffending outcomes. Notably, only Hanby (2013) and Lloyd (2015) have examined change in strengths over time, although both used the same sample data.

\section{Multi-wave Studies Assessing Dynamic Risk and Justice-Involved Men}

Several multi-wave studies have examined change in dynamic risk factors among justice-involved men. Studies conducted by Brown et al. (2009), Howard and Dixon (2013), and Jones et al. (2010) will be discussed in detail. The study conducted by Morgan et al. (2013), however, used a less rigorous approach; although re-assessment information was captured over seven timepoints, point biseral correlations were used to assess the relationship between the last scores and failure, and initial scores and failure. As such, this study does not incorporate change information over three or more reassessment periods and will not be discussed in detail.

Brown et al. (2009) examined the extent to which dynamic factors add to the prediction of recidivism over and above static risk factors. Using a sample of 136 justiceinvolved men released from federal institutions in Ontario, Canada (assessed at prerelease, 1-month post-release, and 3-month post-release), Cox regression with timedependent covariates and receiver operating characteristics (ROC) analyses revealed the 
re-assessment of dynamic risk factors significantly predicted recidivism over and above static risk factors alone (i.e., $\mathrm{AUC}=.89$ vs. $\mathrm{AUC}=.81, p<.01)$. Jones et al. (2010) expanded on this study by incorporating an additional timepoint (6-month post-release) and used a longer follow-up time (6.5 years). Results remained consistent with the previous study with dynamic re-assessments providing the strongest predictive validity; the findings were similar regardless of whether the assessments were based on the researcher's judgment $(\mathrm{AUC}=.86)$ or that of parole officers $(\mathrm{AUC}=.83)$. Although $\mathrm{Cox}$ regression with time-dependent covariates is useful for comparing the predictability of models using data from different timepoints and can inform the predictability of incremental change among assessments, it does not examine an offender's overall rate of change and how that change predicts reoffending.

Finally, although the study by Howard and Dixon (2013) included a small proportion of women ( $13 \%$ of sample), the authors did not disaggregate results by gender. They used a large multi-wave dataset of 196,493 cases on community supervision in England and Wales, who were assessed between 1 and 12 times, over a 48-month period. Cox regression with time dependent covariates was used to examine the predictive validity of the Offender Assessment System (OASys; Howard, Clark \& Garnham, 2006) Violence Predictor (OVP) using repeated assessments. Change in OVP scores were found to significantly predict violent reoffending $($ Hazard Ratio $=1.06)$ and homicide/wounding reoffending $($ Hazard Ratio $=1.08)$, indicating that change in scores were found to improve prediction over initial scores. Cox regression with time dependent covariates has been found to be an effective method to handle multi-wave measures assessed at irregular time periods (Yang, Guo, Olver, Polaschek, \& Wong, 2017). 
However, this approach does not allow for the examination of how individuals change or how patterns of change differ for individuals.

\section{Multi-wave Studies Assessing Dynamic Risk and Justice-Involved Women}

Greiner et al. (2015) was the only study to assess the relationship between change in dynamic risk scores and reoffending for women. Using a similar statistical approach to Brown et al. (2009), the study conducted by Greiner et al., (2015) examined how dynamic factors change across four timepoints, collected at 6-month intervals, and how dynamic re-assessments improve predictive accuracy. Using a sample of 497 justiceinvolved Canadian women released into the community from federal custody, they found that several dynamic risk factors measured using the Community Intervention Scale (Dowden et al., 2001) including employment, marital/family, community functioning, personal/emotional, criminal associates, and criminal attitudes significantly decreased among offenders who refrained from reoffending. However, Cox regression with timedependent covariates and ROC analyses revealed that while all seven dynamic risk predictors were significantly related to survival time, the best fit model at Time 1 included two variables — associates and attitudes $(\mathrm{AUC}=.66)$. In contrast, the best fit model at the last assessment before failure also included two variables - associates and employment needs $(\mathrm{AUC}=.70)$. Notably, the last assessment before failure was more predictive than Time 1. Although results from these studies supported the use of dynamic re-assessment, these studies are limited in that they incorporated gender-neutral variables and did not include more gender-responsive or strengths variables. 


\section{Multi-wave Studies Assessing Dynamic Strengths}

To date, there have only been two multi-wave studies examining the reassessment of dynamic risks and strengths: Hanby (2013) and Lloyd (2015), both of which used re-assessment data over a 12-month period from the Dynamic Risk Assessment of Offender Re-entry (DRAOR; Serin, 2007) for a sample of 3,498 offenders on parole from New Zealand (246 women, 3,249 men). Hanby (2013) used Hierarchical Linear Growth Modelling (HLGM) and Cox regression survival analysis to assess monthly re-assessments of the stable, acute, and protective domains. Results demonstrated that, over time, dynamic risk variables decreased whereas protective factors increased, however, in the month prior to reoffending, an offender's strength scores tended to drop. Cox regression results indicated that later assessments had lower predictive accuracy than earlier assessments. Specifically, monthly average stable risk scores predicted reconvictions immediately following parole start and over the 12 -month re-assessment period; however, monthly average protective factor scores predicted reconvictions for only the first 4 months after the start of parole. Finally, monthly average acute risk scores were not predictive of reconvictions in any of the re-assessment over 12 months following start of parole. Although assessing how scores at each timepoint predict reconvictions, this study did not explicitly examine how change in scores predicted reconvictions.

In contrast, Lloyd (2015), who used the same dataset as Hanby (2013) examined whether proximal DRAOR stable, acute and protective domain assessments were more predictive than distal assessments using a discrete-time hazard model (which is a variant of logistic regression models). Notably, three models were compared: model one 
including only static risk scores from the Risk of Reconviction*Risk of Re-Imprisonment scale (RoC*RoI; Bakker, O’Malley, \& Riley, 1998), model two including static scores and initial stable, acute, and protective domain scores, and model three including static scores and change scores for the stable, acute, and protective domains. Overall, results demonstrate that the model including initial stable, acute, and protective scores added incrementally to the prediction of static risk scores $\left(\chi^{2}=158.85, p<.001\right)$. However, the model including change scores of the stable, acute, and protective domains added incrementally to the prediction of static risk scores to a higher degree $\left(\chi^{2}=294.86, p<\right.$ .001). Upon examining the individual domain scores independently, it was found that incorporating change scores improved prediction for all the domains - stable, acute and protective above and beyond the initial scores.

Overall, the results from these studies demonstrate that the incorporation of dynamic risk factors, in combination with static risk factors, improves the prediction of recidivism. However, most of these studies tend to assess incremental change among timepoints (e.g., Brown et al., 2009; Jones et al., 2010), rather than examine how overall change over time is related to reoffending outcomes. The majority of this research has also focused exclusively on men or have failed to disaggregate results by gender, and with the exception of Hanby (2013) and Lloyd (2015) research has focused solely on the dynamic nature of risk factors rather than strengths. As such, additional longitudinal reassessment data is needed to clarify the relationship between dynamic risks, strengths, and reoffending outcomes among samples of men and women. 


\section{Using the Service Planning Instrument to Assess Change Over Time}

To assess offender change in dynamic factors over time, re-assessment information is required. One assessment tool that assesses various dynamic factorsincluding risk and strength factors, is the Service Planning Instrument (SPIn; Orbis Partners, 2003). The SPIn incorporates a variety of dynamic factors from various content domains and is also considered to be a gender-informed tool, whereby both genderneutral and gender-responsive items are considered. Another important aspect of the SPIn is that many items are measured on a Likert scale rather than dichotomously (yes/no), which improves the tool's ability to capture change over time (i.e., tool is more sensitive to change). For these reasons, the SPIn was determined to be an appropriate tool to use to capture change in dynamic scores among justice-involved men and women.

\section{Purpose of Study 1}

The main purpose of this study is to examine the dynamic nature of genderinformed risk and strength scores assessed by the SPIn. To date, no studies have assessed how SPIn-assessed total dynamic risk and strengths change over time or how change patterns in SPIn-assessed total dynamic risk and strength scores predict recidivism. The current study uses multi-wave re-assessment data with a large sample of adults on community supervision to assess: 1) patterns of change in total dynamic risk and strength scores and how these changes may differ among men and women; and 2) the relationship between changes in total dynamic risk and strength scores and reoffending outcomes for men and women, respectively.

Research Question 1: How do SPIn-assessed dynamic risk factors change over time and do these changes vary by gender? Previous research has found that over time, 
women (e.g., Greiner et al., 2015) and men (e.g., Brown et al., 2009; Jones et al., 2010) who remain successful in the community have dynamic risk scores that decrease over time.

Hypothesis 1: It is hypothesized that over time, total dynamic risk scores will decrease for both men and women who remain successful in the community.

Research Question 2: How do SPIn-assessed dynamic strengths change over time and do changes vary by gender? One study, conducted by Hanby (2013) assessed strengths over time and found that as time progressed strengths increased for those who did not reoffend.

Hypothesis 2: It is hypothesized that over time, total dynamic strength scores will increase for both men and women who remain successful in the community.

Research Question 3: Does the rate of change in SPIn-assessed dynamic risk and strength factors predict recidivism for both men and women? To date, no studies have assessed how change patterns in SPIn-assessed dynamic strength and risk factors predict recidivism.

Hypothesis 3: Despite the limited research it is nonetheless expected that individuals whose dynamic risk domain scores decrease over time will be less likely to reoffend than those whose dynamic risk domain scores remain stable or increase.

Similarly, it is expected that individuals whose dynamic strength domain scores increase over time will be less likely to reoffend than those whose scores decrease or remain stable. Similar results are expected for both men and women. 


\section{Method for Study 1}

\section{Participants}

Larger SPIn dataset. This study uses archival SPIn data provided by Orbis

Partners Inc. Orbis Partners Inc. is a Canadian consulting firm based in Ottawa, Ontario that specializes in implementing evidence-based services for clients who are at-risk for justice involvement, including assessments, programs, case work training, and research and evaluation. The original database includes a total of 118,149 SPIn assessments (mean number of assessments per participant is $2.9 ; S D=1.6$; median $=3.0$; range $=1-19$ ) corresponding to 61,846 adult clients on provincial community supervision across Alberta, Canada. Recidivism data associated with the database is further organized into a fixed 3-year and a fixed 6-year follow-up period. The 3-year follow-up sample is made up of 31,477 clients $(79.5 \%$ men $[n=25,012] ; 20.5 \%$ women $[n=6,465])$ and the 6 -year follow-up sample is made up of 4,959 clients $(80.8 \%$ men $[n=4,007] ; 19.2 \%$ women $[n$ =952]). The data for the current study was extracted from the fixed 3-year follow-up sample, where the mean number of assessments was $3.06(S D=1.26$; median $=3.0$; range of 0-5 Full Assessments, as some individuals just had a completed SPIn Pre-Screen assessment). It is important to note that research conducted by Jones, Brown, Robinson, and Frey (2015), and Brown, Wanamaker, Wagstaff, and Robinson (2020), have used samples subsumed within this larger dataset. ${ }^{4}$

\footnotetext{
${ }^{4}$ Of note, the study conducted by Brown and colleagues (2020) used initial SPIn assessment data from 20,537 men and women, not disaggregated by gender, to examine the interaction between total dynamic risk and total strength scores. Jones and colleagues (2015), in contrast, only used pre-screen SPIn assessment data from 3,656 men and women to examine the extent to which the consideration of strengths enhanced the predictive validity of risk assessment protocols, using an 18-month follow-up.
} 
Sample used in current study. The sample consisted of a cohort of justiceinvolved men and women who initially started community supervision between 2009 and $2012($ Mdn year $=2012)$. Each case in the sample was serving a provincial sentence for an index offence(s) with some cases starting their sentence immediately under community supervision and others starting community supervision post-release from a provincial correctional facility. The final sample used to assess change was comprised of 14,830 justice-involved cases (11,953 men and 2,877 women), and contained between 3 and 5 completed SPIn assessments for men $(M=3.9, S D=.87$, median $=4.0)$ and for women $(M=3.8, S D=.86$, median $=3.0)$. Each man and woman included in the current study had at least three completed SPIn assessments, as this was the median number of assessments for women and change research has indicated that a minimum of three assessments are required (Babchishin, 2013). There was a fixed three-year follow-up period from the time of the initial assessment. Notably, men and women with initial assessments that did not occur within 90 days of the start of supervision were excluded from analyses, as assessment information later than this timepoint is not reflective of true initial scores and thus, the change parameters would not be an accurate representation of that individuals change trajectory (see Figure 1 for sample inclusion criteria). 


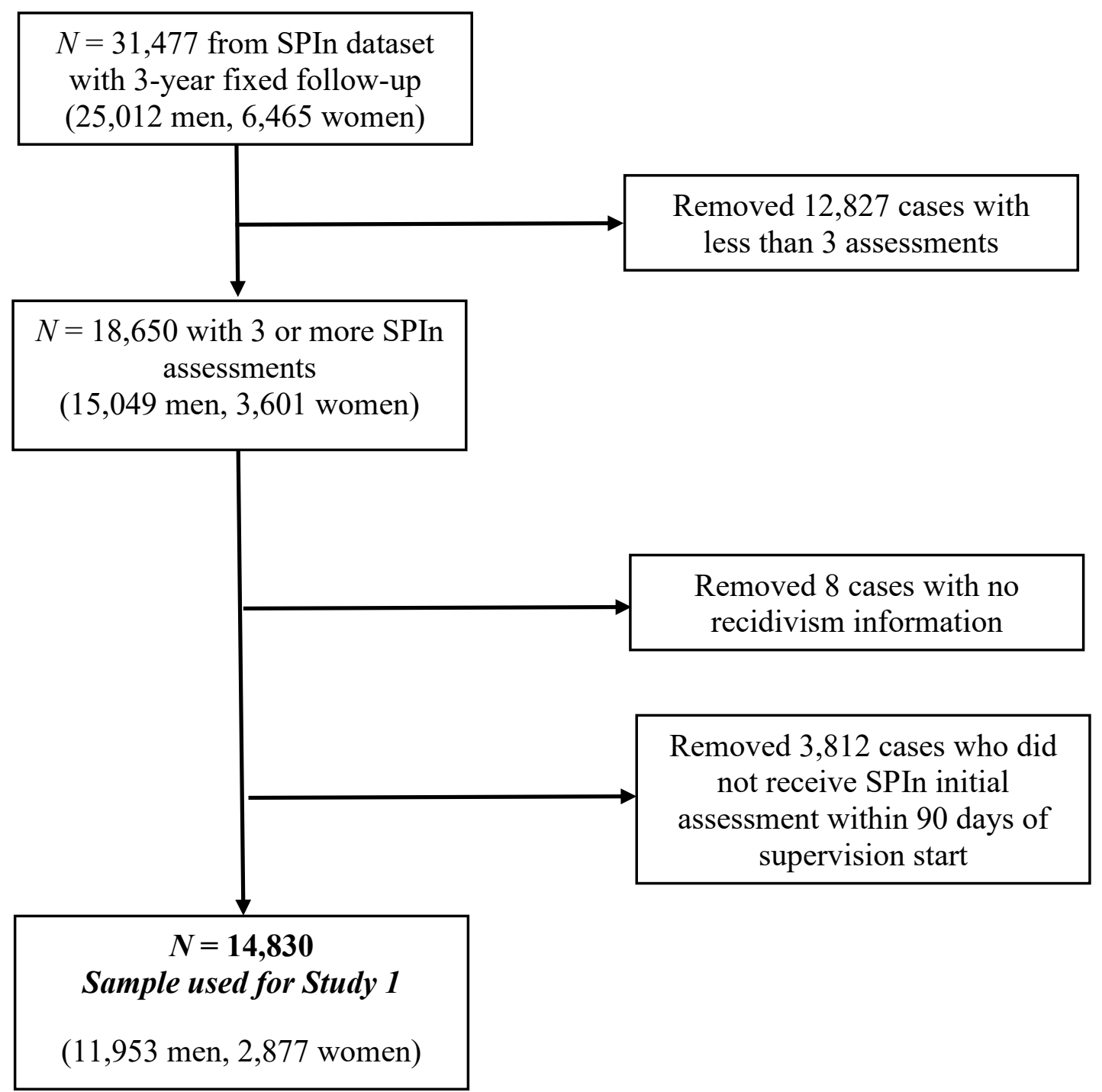

Figure 1. Participant flow chart for study 1.

Descriptives. The sample was comprised of 11,953 men and 2,877 women. Based on SPIn Full Assessment static risk scores, the majority of the men and women were low risk $($ men $=49.7 \%$; women $=57.6 \%)$, about a third were moderate risk $($ men $=37.1 \%$; women $=34.7 \%)$, with very few who scored high risk $($ men $=12.8 \%$; women $=6.9 \%)$. As Table 1 illustrates, men were slightly older than women (33.6 vs. 32.9 years old). A higher proportion of women were Indigenous (28.8\%) compared to the proportion of men 
$(16.6 \%)$, and more women tended to have at least one mental health flag than the men $(64.8 \%$ vs. $44.5 \%)$.

In terms of index offences, a higher proportion of women committed a nonviolent offence (37.6\% vs. $23.9 \%$ ), and an administration of justice offence (6.8\% vs. $5.2 \%)$, whereas a higher proportion of men committed a violent offence $(42.5 \% \mathrm{vs}$. $29.8 \%$ ) and a sexual offence (4.2\% vs. 1.3\%). For the SPIn initial Full Assessment, there were no significant gender differences on total dynamic risk score, however, men tended to score higher than women on both the total static risk score (25.0 vs. 20.6) and the total dynamic strength score (27.8 vs. 26.9). Finally, in terms of recidivism, there was significant differences among men and women for each of the various outcomes. Specifically, a significantly higher proportion of men than women had a new violent charge (16.2\% vs. $10.1 \%)$, a technical violation ( $22.8 \%$ vs. $19.7 \%)$, any new charge (29.1\% vs. $24.9 \%)$, and any negative outcome (36.2\% vs. $32.4 \%)$ within 3 -years post initial SPIn Full Assessment. 
Table 1

Descriptive Information for Study 1, Split by Gender

\begin{tabular}{|c|c|c|c|c|c|c|c|}
\hline \multirow[b]{2}{*}{ Variable } & \multicolumn{2}{|c|}{$\begin{array}{c}\text { Men } \\
(N=11,953)\end{array}$} & \multicolumn{2}{|c|}{$\begin{array}{c}\text { Women } \\
(N=\mathbf{2 , 8 7 7})\end{array}$} & \multirow[b]{2}{*}{$t$} & \multirow[b]{2}{*}{$\chi^{2}$} & \multirow[b]{2}{*}{$d$} \\
\hline & $M(S D)$ & $\%(n)$ & $M(S D)$ & $\%(n)$ & & & \\
\hline \multicolumn{8}{|l|}{ Demographics } \\
\hline Age & $33.6(11.4)$ & & $32.9(10.8)$ & & $2.73^{*}$ & & .06 \\
\hline Indigenous & & $16.6(1,988)$ & & $28.8(829)$ & & $223.68 * *$ & .25 \\
\hline Any custody & & $26.4(3,155)$ & & $18.6(536)$ & & $74.78 * *$ & .14 \\
\hline $\mathrm{MH}_{\text {flag }}{ }^{1}$ & & $44.5(5,316)$ & & $64.8(1,867)$ & & $393.60 * *$ & .33 \\
\hline \multicolumn{8}{|l|}{ Index Offence ${ }^{2}$} \\
\hline Sexual & & $4.2(502)$ & & $1.3(37)$ & & $56.21 * *$ & .12 \\
\hline Violent & & $42.5(5,079)$ & & $29.8(856)$ & & $156.75 * *$ & .21 \\
\hline Non-violent & & $23.9(2,860)$ & & $37.6(1,081)$ & & $221.32 * *$ & .25 \\
\hline Admin. & & $5.2(623)$ & & $6.8(195)$ & & $10.91 * *$ & .05 \\
\hline Other & & $12.0(1,436)$ & & $11.5(330)$ & & .65 & .01 \\
\hline \multicolumn{8}{|l|}{ Full Assessment info ${ }^{3}$} \\
\hline Static risk & $25.0(18.8)$ & & $20.6(16.0)$ & & $9.96 * *$ & & .25 \\
\hline Dynamic risk & $19.3(15.8)$ & & $18.7(15.9)$ & & 1.34 & & .04 \\
\hline Dynamic Strength & $27.8(16.4)$ & & $26.9(16.5)$ & & $3.99 * *$ & & .05 \\
\hline \multicolumn{8}{|l|}{ Recidivism $^{4}$} \\
\hline Any negative ${ }^{5}$ & & $36.2(4,326)$ & & $32.4(932)$ & & $14.61 * *$ & .06 \\
\hline Technical & & $22.8(2,729)$ & & $19.7(568)$ & & $12.79 * *$ & .06 \\
\hline New violent & & $16.2(1,940)$ & & $10.1(292)$ & & $67.06 * *$ & .14 \\
\hline Any new & & $29.1 \quad(3,478)$ & & $24.9(716)$ & & $20.27 * *$ & .07 \\
\hline
\end{tabular}

Note. $M=$ Mean. $S D=$ Standard deviation. $t=\mathrm{t}$-test. $\chi^{2}=$ Chi square. $d=$ Cohen's d effect size. ${ }^{1} \mathrm{MH}=$ Mental Health. ${ }^{2}$ Non-violent $=$ property, theft, fraud, drug offences, impaired driving. Admin $=$ technical violations and administration of justice offences. Other $=$ traffic and other offences. ${ }^{3}$ Assessment info $=$ based on initial assessment information. ${ }^{4}$ Recidivism $=$ based on 3-year follow-up information. ${ }^{5}$ Any negative $=$ includes any technical violations and/or new charges. Missing data on the index offence was $12.2 \%$ for men and $13.1 \%$ for women. ${ }^{*} p<.01,{ }^{* *} p<.001$ 


\section{Measures}

The Service Planning Instrument (SPIn) - Full Assessment. The SPIn (Orbis Partners, 2003) is a risk, need, and strength assessment and case management planning instrument. This tool can be used with adults in both institutional and community-based justice settings, and incorporates information from a semi-structured interview and fileinformation. Notably, the SPIn was developed based on the Youth Assessment and Screening Instrument (YASI; Orbis Partners, 2000), which was adapted from the Case Management Assessment Protocol (CMAP; Barnoski, 2003); a risk, need, and strengths juvenile assessment tool developed for justice-involved youth in Washington State.

The SPIn is considered a gender-informed risk assessment tool, where both gender-neutral and gender-responsive items are included, however, the tool was not developed from the ground-up specifically for women. Importantly, SPIn users are required to complete a total of four days of training - two days of training on how to administer the SPIn, and another two days of training on how to apply the SPIn results to case planning and case management.

In Alberta, policy stipulates that initial SPIn assessments should be conducted within 45 days of the start of court ordered community supervision or release from custody. SPIn re-assessments should be completed every 6 months; however, in practice this could vary based on how often a client is seen (which could be based on risk level or resources). The SPIn is not required if the supervision period is 3 months or less, if the court order does not have a reporting condition placed on the client, if the client is convicted of a new charge(s) while under supervision and there is already a recent 
completed assessment, and if the client will be leaving the province within 45 days of the start of supervision.

The full SPIn assessment contains 90 items; 35 are used to calculate a Pre-Screen risk score and Pre-Screen strength score (11 items). While the Pre-Screen strength score is rarely used in practice, the Pre-Screen risk score is used for making initial supervision classification level decisions. The current study, however, only uses the SPIn Full Assessment. The 90 items are organized within 11 domains: Criminal history, response to supervision (e.g., institutional misconducts, violations), aggression, substance use, social influences (e.g., negative and prosocial peers), family, employment and education, attitudes, social and cognitive skills, stability (e.g., living situation, financial situation), and mental health. As Figure 2 demonstrates, most domains contain both static and dynamic items but there are exceptions: criminal history, and response to supervision are comprised entirely of static items. The mental health flag has only one dynamic item, but does not contribute to the total static or total dynamic risk score. In contrast, social influences, attitudes, and social/cognitive skills are comprised entirely of dynamic items. The majority of domains include the assessment of both strength and risk itemshowever, criminal history, response to supervision, mental health flag, and substance use domains do not contain any strength items. 


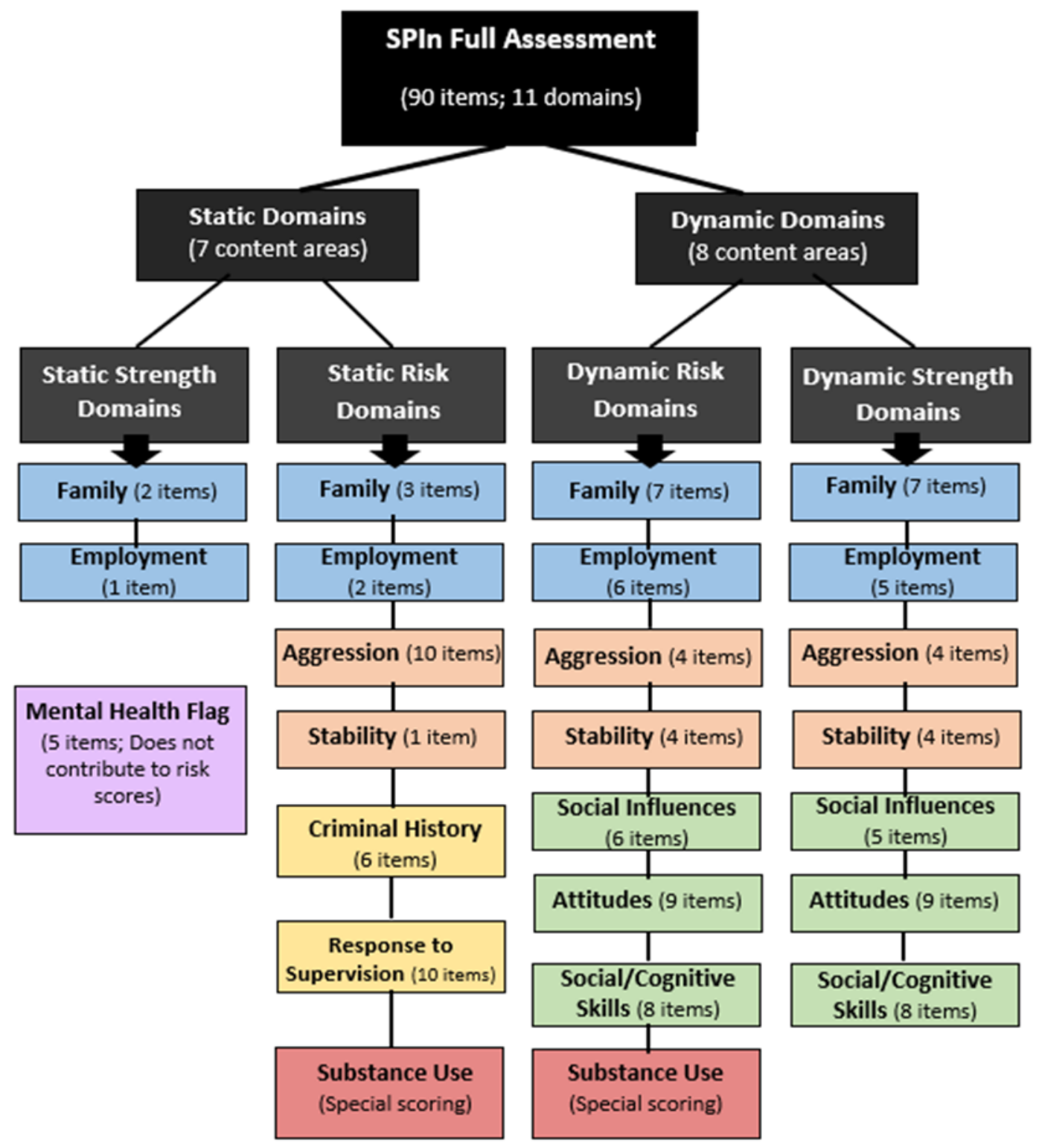

Figure 2. Breakdown of SPIn full assessment dynamic and static risk and strength domains.

Further, 32 items ${ }^{5}$ that contribute to the various dynamic domain scores are scored in a poled 5-point Likert fashion such that they are scored as strengths or risks. For

\footnotetext{
${ }^{5}$ Each domain also contains a dynamic, poled item assessing motivation to address that particular risk; however, this item is not included in the calculation of domain or total dynamic scores. Rather, these scores are used to gage motivation levels for case management purposes.
} 
example, as seen in Figure 3, risk and strength represent opposite poles of the construct being assessed - in this case, current intimate relationships. Specifically, this item can be rated on the risk side as either a 2 (High degree of instability and conflict, offender expresses high dissatisfaction) or a 1 (Some conflict and dissatisfaction evident in the relationship). Conversely, it can be rated on the strength side as a 2 (High degree of stability, satisfaction and commitment to the relationship) or a 1 (Stability of the relationship evident, offender expresses satisfaction). Finally, the item can instead be rated as neutral (score of 0 ) which indicates that the item is neither a strength nor a risk factor (Minimal satisfaction in relationship or no current marital relationship).

\begin{tabular}{|c|c|c|c|c|}
\hline O -- -- & 0 -- & $\bullet 0$ & $0+$ & $0++$ \\
\hline $\begin{array}{l}\text { High degree of } \\
\text { instability and } \\
\text { conflict, offender } \\
\text { expresses high } \\
\text { dissatisfaction. }\end{array}$ & $\begin{array}{l}\text { Some conflict and } \\
\text { dissatisfaction } \\
\text { evident in the } \\
\text { relationship }\end{array}$ & $\begin{array}{l}\text { Minimal } \\
\text { satisfaction in } \\
\text { relationship (or } \\
\text { no current } \\
\text { marital } \\
\text { relationship) }\end{array}$ & $\begin{array}{l}\text { Stability of } \\
\text { relationship } \\
\text { evident, } \\
\text { offender } \\
\text { expresses } \\
\text { satisfaction }\end{array}$ & $\begin{array}{l}\text { High degree of } \\
\text { stability, } \\
\text { satisfaction and } \\
\text { commitment to } \\
\text { the relationship }\end{array}$ \\
\hline
\end{tabular}

Figure 3. Example of a SPIn item (current intimate relationship).

Therefore, while many domains contain both strength and risk scores, these strength and risk scores are often rated using the same items - that is, one single item produces a risk and a strength score whereby if the individual scores a 1 or a 2 on the risk side, they automatically get a 0 on the strength side of the item (and vice versa). For example, the attitudes dynamic risk and strength domains are both made up of 9 items; however, these domain scores are based on the same 9 items. Therefore, if someone scores a 1 or 2 on risk for all 9 attitude items, they would automatically have a score of 0 on strength for all 9 attitude items. 
The remaining 41 items (as well as 5 items from the mental health domain and 3 items from the substance use domain ${ }^{6}$ ) are not scored in a poled fashion. Rather they are scored as pure risk factors or pure strength factors (i.e., either dichotomous yes/no items, or "check all that apply" items, such as gang association or, for an example of strength, partner has pro-social influence).

Individual SPIn items are weighted using the Nuffield method (Nuffield, 1982) and then summed into the following scores: The SPIn Pre-Screen risk score is comprised of 36 items and the plausible range is 0 to $67+$ (see Appendix A), whereby scores of 1 to 30 are considered low, scores of 31 to 66 are considered moderate, and scores of 67 or higher are considered high. The Pre-Screen strength score is comprised of 11 items and the plausible range is 0 to $15+$, whereby scores of 1 to 7 are considered low, scores of 8 to 14 are considered moderate and scores of 15 or more are considered high.

In the Full Assessment, there are two static strength sub-scores and seven static risk sub-scores which include the following domains and score ranges: family strength (0-4), employment strength (0-3), family risk (0-9), employment risk (0-9), criminal history risk (0-20), response to supervision risk (0-43), aggression risk (0-41), substance use risk (0-9), and stability risk (0-2). See Figure 2 and Table 1A in Appendix A for number of Pre-Screen items in each domain.

\footnotetext{
${ }^{6}$ The substance use domain assesses 11 different types of substances including: alcohol, marijuana, cocaine/crack, ecstasy and other club drugs, heroin, hallucinogens, inhalants, amphetamines, methamphetamines, prescription drug misuse, and any other substances. Each substance is assessed on the times used in the last three months, whether it disrupts functioning, whether it contributes to behaviour, and whether there are attempts to cut back. As such, the substance use dynamic risk score is the sum of the single highest value on Times used in last three months item, plus the single highest value on Disrupts Function, plus the highest value on Contributes to Behaviour, minus one for any instance of Attempts to cut back. The substance use static risk is based on the youngest age at which the individual used any drug.
} 
In addition, there are seven dynamic strength sub-scores which include the following domains and score ranges: family (0-14), employment (0-12), social (0-15), aggression (0-8), attitudes (0-14), cognitive/social skills (0-18), and stability (0-7).

Finally, there are eight dynamic risk sub-scores which include the following domains and score ranges: family (0-26), employment (0-14), substance use (0-28), aggression (0-8), social (0-26), attitudes (0-14), social/cognitive skills (0-18), and stability (0-13). See Figure 2 and Table 1A in Appendix A for number of items in each domain.

Raw scores are translated into low, moderate, and high levels based on jurisdictional norms, whereby estimates of recidivism rates associated with each classification level of risk and strength were set in accordance with the needs of Alberta, rather than empirically-derived recidivism rates which may not correspond well with Alberta (Jones \& Robinson, 2017a). These norms were determined by a preliminary recidivism study based on a 6-month follow-up period.

Finally, the various dynamic domain scores can be aggregated to calculate a total dynamic risk score and a total dynamic strength score, and static risk domains can be aggregated to calculate a total static risk score ${ }^{7}$. The total dynamic risk scores range from 0 to $75+$, and total dynamic strength scores range from 0 to $57+$. See Table 2 for a breakdown of score ranges corresponding to the different classification levels.

\footnotetext{
${ }^{7}$ The SPIn typically refers to strengths as Protective Factors however, despite some preliminary findings, there has not been enough evidence to indicate that the strengths assessed in the SPIn indeed demonstrate a Protective Effect, the current study used the term Strength to denote positive, prosocial factors. In contrast, the term Dynamic Risk will be used instead of Criminogenic Need to avoid confusion and ensure a clear distinction between negative, antisocial and positive, prosocial factors.
} 
Table 2

Score Ranges Corresponding to Classification Levels on Full Assessment Total Dynamic Risk and Strength Scores

\begin{tabular}{ccc}
\hline & Dynamic Risk & $\begin{array}{c}\text { Dynamic Strength } \\
\text { Classification Level }\end{array}$ \\
\hline Scores & 0 & 0 \\
Low & $1-16$ & $1-11$ \\
Low-Moderate & $17-26$ & $12-21$ \\
Moderate & $27-38$ & $22-30$ \\
Moderate-High & $39-54$ & $31-41$ \\
High & $55-74$ & $42-56$ \\
Very High & $75+$ & $57+$ \\
\hline
\end{tabular}

In the current study, the eight dynamic risk domain scores and the seven dynamic strength domain scores will be used to assess statistical assumptions, including measurement invariance. However, for the purpose of the main analyses, only the total dynamic risk, total dynamic strength, and total static risk scores (control variable) from the full SPIn assessment will be included. Please see Table 1A, 2A and Figure 1A in Appendix A for a complete breakdown of the SPIn individual items, sample items from each dynamic domain, and an example of a SPIn wheel—used to display risk and strength scores.

Psychometric properties of the SPIn. Research on the psychometric properties of the SPIn, although promising, is largely in its infancy (Wanamaker et al., 2018). For instance, the interrater reliability of the SPIn has not yet been examined (Jones \& Robinson, 2018). To date only three studies have examined the validity of the SPIn. First Jones et al. (2015) used a sample of 3,656 men and women on community supervision in Alberta to examine the predictive validity of the SPIn Pre-Screen risk and strength scores 
using an 18-month follow-up. Notably, the sample used by Jones and colleagues (2015) is subsumed within the sample from the current dissertation. This study found that the SPIn Pre-Screen accurately predicts new charges across an 18-month follow-up (AUC = .77), irrespective of gender (men $\mathrm{AUC}=.77$; women $\mathrm{AUC}=.77$ ), and ethnicity (Indigenous $\mathrm{AUC}=.75$; non-Indigenous $\mathrm{AUC}=.76$ ).

Further, a more recent report prepared for Alberta Community Corrections by Jones and Robinson (2017a), used a large sample of men and women from Alberta which included 46,794 clients with a 1-year follow-up, 31,477 clients with a 3-year follow-up, and 4,959 clients with a 6-year follow-up. Importantly, both the data from the Jones et al. (2015) study and data from the current dissertation are subsumed within this larger sample used in Jones and Robinson (2017a). Overall, they found that the SPIn Pre-Screen risk total significantly predicted various offender outcomes at moderate to high levels of accuracy, with AUCs ranging from .64 (for any conviction) to .75 (for any custody) at 1year follow-up. Violent recidivism was found to have an AUC of .69 and technical violations had an AUC of .73. The AUC values remained relatively consistent at the 3year follow-up (AUCs ranged from .64 to .74) and 6-year follow up mark (AUCs range .64 to .74). Furthermore, the predictive accuracy of the SPIn Pre-Screen risk total was relatively equivalent across ethnicity and gender subgroups (Jones \& Robinson, 2017a). The SPIn Pre-Screen strength total was predictive of all offender outcomes, but with smaller effect sizes, for the 1-year follow-up (AUC range .61 to .68), 3-year follow-up (AUC range .59 to .67), and 6-year follow-up (AUC range .62 to .68). The AUCs were comparable across gender and ethnicity, although the Indigenous samples had smaller effect sizes across all outcomes. 
In addition, this study also examined the predictive validity of the SPIn Full Assessment using a sample of 28,423 men and women over a 3-year follow-up period (once again the sample used in the current dissertation is subsumed within this larger sample). Overall, they found that the total dynamic risk scores predicted various negative outcomes, with small to moderate effects (AUCs ranged from .61 to .69); the effects were comparable for men (AUCs ranged from .61 to .68) and women (AUCs ranged from .61 to .70). Although the total dynamic strength scores attained on the SPIn full assessment predicted various negative outcomes, the effects were considerably smaller with AUCs ranging from .53 to .58 . These findings were similar for both genders with AUCs ranging from .53 to .58 for the men and .52 to .59 for the women (Jones \& Robinson, 2017a).

Finally, a report conducted on the validity of the SPIn for Clark Country, Washington Adult Probation Services used a sample of 1,233 men and 375 women followed over a 3-year period (Jones \& Robinson, 2017b). Overall, they found that both the SPIn Full Assessment total dynamic risk score and total strength score was predictive of new convictions, both with an AUC of .65. This study also looked at the predictive validity of the individual dynamic domains for men and women independently. For men, dynamic risk domains predicted new convictions with AUCs that ranged from .56 to .66, and dynamic strength domains predicted new convictions with AUCs that ranged from .58 to .63 . For women, dynamic risk domains predicted new convictions, with AUCs ranging from .54 to .65 , and dynamic strength domains predicted new convictions with AUCs that ranged from .58 to .63 .

The current study found that for the initial SPIn Full Assessment, internal consistency was good for the total dynamic risk domain for the overall sample $(\alpha=.74)$ 
and for men $(\alpha=.74)$ and women $(\alpha=.76)$, independently. For the dynamic strength domain, internal consistency was found to be good for the overall sample $(\alpha=.84)$ and for men $(\alpha=.84)$ and women $(\alpha=.85)$, independently. See Appendix B for the internal consistencies of the various dynamic risk and strength domain scores for the overall sample, and for men and women independently.

Criminal outcome. Four dichotomous (yes/no) measures of reoffending were used based on a data file containing reoffence records from the department of the Alberta Justice and Solicitor General, which was provided to Orbis Partners. The data file contained records on any new offences committed post-release whereby the individual made recontact with correctional services in the province of Alberta. The four outcomes include any negative outcome, any new charges, any violent charges, and any technical violations, each described in detail:

(1) Any negative outcome(s): this includes any technical violation charges (breach of condition, failure to comply, and failure to appear) as well as any criminal justice system re-contract. This includes new charges, such as non-violent charges (property offences such as mischief, theft, break and enter, forcible entry, and possession of stolen property, fraud, drug-related offences, including possession and trafficking, obstruction, trespassing, and causing a disturbance), sexual charges, and violent charges (such as uttering threats, all forms of assault including causing bodily harm, assault with a weapon, assault of a peace officer, and simple assaults, any weaponrelated offences, including pointing a firearm, possession and careless storage, harassment, robbery, dangerous driving or operation causing bodily harm, damage by arson, and any murder charges). 
(2) Any new charge(s): this outcome excludes any technical violations, but includes any new charges that are non-violent, sexual, or violent in nature (see any negative outcome for details of charges corresponding to each charge type).

(3) Any new violent charge(s): this outcome excludes any technical violation, as well as any new non-violent charges and any new sexual charges. Violent charges includes uttering threats, all forms of assault (including causing bodily harm, assault with a weapon, assault of a peace officer, and simple assaults), any weapon-related offences (including pointing a firearm, possession and careless storage), harassment, robbery, dangerous driving or operation causing bodily harm, damage by arson, and any murder charges.

(4) Any new technical violation(s): this outcome consists of any breaches of courtordered or community supervision conditions resulting in a failure to comply, or failure to appear. This outcome does not include any new offence charges.

Each of these outcomes were assessed over a 3-year fixed follow-up from the time of completion of the initial SPIn Full Assessment, which was assessed within 90 days of their start of community supervision. The start of community supervision in this context refers to those who either started court-ordered probation, started a conditional sentence order, or started community supervision upon release from provincial custody.

\section{Analytic Approach}

Prior to running analyses, data screening, data cleaning, and assumption checking were conducted using Statistics Package for the Social Sciences (SPSS) version 25 (IBM, 2017). This was followed by analyses assessing measurement invariance (a preliminary step to change analysis) conducted using MPlus Software Version 8.3 (Muthén \& 
Muthén, 1998-2017). Analyses assessing patterns of change and the relationship to recidivism was conducted using SAS software version 9.4 (SAS Institute Inc., 2016). See Table 1C in Appendix $\mathrm{C}$ for a breakdown of analyses.

\section{Data Analyses and Results}

The following section describes the statistical approach and results as follows: (1) Data screening and basic assumption checking, (2) Measurement invariance, (3) Patterns of change, and, (4) Rates of change and recidivism.

\section{Data Screening and Basic Assumption Checking}

Prior to running analyses, the data were screened for missing values, outliers, normality, linearity, and multicollinearity. Missing assessment information across timepoints is presented in Table 3. The large amount of missing assessment information at later timepoints is due to time of measurement (e.g., participant recidivated and thus was not assessed again, follow-up time was not long enough). However, this also includes assessment information that is truly missing; that is, assessment information not present, despite success in the community with adequate follow-up time. The amount of missing data that is deemed true missing across each of the assessment timepoints is minimal, comprising less than $4 \%$ (range: $0 \%$ to $3.6 \%$ ). The pattern of missing data was a function of time; that is, data across timepoints were missing because it was not available due to length of follow-up or success in the community. As such, the data is considered to be missing at random (MAR), which means there is a systematic relationship between the missingness of values and available data, as opposed to missing completely at random (MCAR), which indicates that there is no relationship between the missingness of data and any values. 
Table 3

Missing Data on Full SPIn Assessments Across Five Timepoints

\begin{tabular}{lccccc}
\hline & \multicolumn{2}{c}{$\begin{array}{c}\text { Men } \\
(N=11,953)\end{array}$} & & \multicolumn{2}{c}{$\begin{array}{c}\text { Women } \\
(N=2,877)\end{array}$} \\
\cline { 2 - 3 } \cline { 5 - 6 } Assessment Number & $n$ & $\%$ & & $n$ & $\%$ \\
\hline Assessment \#1 & 0 & 0.0 & & 0 & 0.0 \\
Assessment \#2 & 429 & 3.6 & & 81 & 2.8 \\
Assessment \#3 & 25 & 0.1 & & 4 & 0.1 \\
Assessment \#4 & 6153 & 51.5 & & 1648 & 57.3 \\
Assessment \#5 & 9201 & 77.0 & & 2301 & 80.0 \\
\hline
\end{tabular}

Additionally, assumptions were tested independently for men and women across timepoints. Every possible pairwise plot between dynamic domain scores (risk and strength) and total dynamic scores (risk and strength) indicated linear relationships for the majority of combinations. The assumption of homoscedasticity was also tested and was supported with the exception of the substance use domain which was found to be slightly heteroscedastistic across timepoints and for both genders.

Kolmogorov-Smirnov's test of normality was conducted on each of the dynamic risk and strength domains as well as the total dynamic risk and total dynamic strength scores and was found to be significant $(p<.001)$ in most cases (for both men and women across timepoints), indicating non-normal distributions. These results are not surprising given the large sample size, as even the slightest departure from normality will results in a significant finding. As such, the data was examined using a variety of graphs and visual representations (e.g., histograms, Q-Q plots, Boxplots), as well as skewness and kurtosis values. Visual representations indicated slightly positive to moderately positive skews. 
Upon examining standardized scores, most of the SPIn domain scores were within the critical values of $+/-3.29$ (two-tailed test). Across all timepoints, less than $3.5 \%$ of cases had standardized scores over 3.29 across all dynamic risk and strength domains and total dynamic risk and total dynamic strength scores. None of these cases appeared "unattached" from the rest of the distribution, with the exception of 8 cases with extreme outlying total dynamic risk scores and 9 cases with extreme outlying scores in the substance use domain. As such, these outlying values were brought within range, while maintaining their position in the distribution. Notably these cases represent less than $.01 \%$ of the sample. No transformations were made, given the large sample size.

Mahalanobis' distance was used to identify multivariate outliers within the data. Using a critical value of $39.25(d f=16, \alpha<.001)$, timepoint 1 had 514 cases exceeding the critical value ( $4.7 \%$ of cases), timepoint 2 had 588 cases exceeding the critical value (5.4\% of cases) and timepoint 3 had 594 cases exceeding the critical value $(5.4 \%$ of cases). However, upon further inspection, these cases were deemed to be real cases-not due to data entry or scoring errors. Therefore, as the goal was to examine changes in scores over time and how it may relate to recidivism, and to avoid limiting the generalizability of the study (while maintaining assessment sequences) cases were kept (Tabachnick \& Fidell, 2013).

\section{Measurement Invariance}

The first step to assessing change over time is to examine whether the SPIn meets the measurement invariance assumption. Measurement invariance can be assessed in longitudinal studies to examine if domains in the risk assessment scale (in this case, total dynamic risk and total dynamic strength scores) assess the same construct in the same 
way across time. When there is a violation of measurement invariance (i.e., measurement non-invariance or partial measurement invariance) the measure may not accurately assess true change in scores over time, but rather change in scores may be due to measurement error (Asparouhov \& Muthén, 2009).

There are five main steps to assessing measurement invariance: 1) selecting the appropriate sample for assessing measurement invariance, 2) assessing the statistical assumptions for exploratory factor analysis (EFA), 3) running an initial EFA on the first timepoint to determine the type of rotation to use $\left.{ }^{8}, 4\right)$ running EFAs using the determined rotation type across all timepoints to ensure consistent factor structure across time, and 5) running exploratory structural equation modeling to examine the extent to which factor loadings and factor structure are invariant over time. Measurement invariance is said to exist when the way in which a latent construct is measured is equivalent over time (Vandenberg \& Lance, 2000).

Selection of measurement invariance sample. There are several requirements that need to be met when selecting a sample to assess measurement invariance. First, since the current study is assessing the invariance of the SPIn over time, at least three timepoints are required. Second, timepoints need to be equally distanced over time. That is, timepoints need to occur at fixed intervals, rather than at variable or random timepoints. Finally, there cannot be any missing data across each of the timepoints.

\footnotetext{
${ }^{8}$ The current study required conducting EFAs across timepoints as an initial step to assessing measurement invariance because previous research has not assessed the factor structure of the SPIn.
} 
To account for these requirements, the current study grouped assessments into the following three timepoints: Time 1 = initial assessment (occurring within 90 days of start of supervision), Time $2=3$ to 8 months post initial assessment, Time $3=9$ to 14 months post initial assessment. These timepoints allowed for the least amount of cases to be removed (i.e., a large proportion of the full sample fit into these grouping parameters) and was in-line with SPIn re-assessment protocols, which indicate that the SPIn assessment should be completed every 6 months. In some cases, men and women had more than one SPIn assessment per timepoint. In these instances, rather than systematically selecting an assessment to represent this timepoint (e.g., choosing first, last, midpoint, or averaging the assessments), an assessment was selected randomly. Overall, the final measurement invariance sample resulted in 8,804 men and 2,152 women. Although the use of grouping assessment timepoints resulted in a substantial reduction in sample size, the initial sample was large enough to maintain an adequate sample for testing measurement invariance.

\section{Descriptive information for measurement invariance sample. As Table 4}

illustrates, men were slightly older than women (33.8 vs. 33.1 years old). A higher proportion of women were Indigenous $(27.6 \%)$ compared to the proportion of men (15.7\%), and more women had at least one mental health flag than the men $(65.8 \% \mathrm{vs}$. $45.0 \%$ ). In terms of index offences, a higher proportion of women committed a nonviolent offence (37.2\% vs. $23.2 \%$ ), and an administration of justice offence (6.2\% vs. $4.8 \%$ ), whereas a higher proportion of men committed a violent offence $(43.8 \% \mathrm{vs}$. $30.6 \%)$ and a sexual offence (4.4\% vs. 1.3\%). For the SPIn initial Full Assessment, there were no significant gender differences on total dynamic risk score, however, men tended 
to score higher than women on both the total static risk score (24.8 vs. 20.5) and the total dynamic strength score (27.9 vs. 27.1). Finally, in terms of recidivism, there was significant difference among men and women for each of the various outcomes. Specifically, a significantly higher proportion of men than women were charged with committing a new violent charge (15.0\% vs. $9.8 \%)$, a technical violation $(21.1 \%$ vs. $18.1 \%)$, any new charge (26.9\% vs. $23.4 \%)$, and any negative outcome $(34.0 \%$ vs. 30.7\%) within 3-years after the initial SPIn Full Assessment. 
Table 4

Descriptive Information of Measurement Invariance Sample of Men and Women

\begin{tabular}{|c|c|c|c|c|c|c|c|}
\hline \multirow[b]{2}{*}{ Variable } & \multicolumn{2}{|c|}{$\begin{array}{c}\text { Men } \\
(N=\mathbf{8 , 8 0 4})\end{array}$} & \multicolumn{2}{|c|}{$\begin{array}{c}\text { Women } \\
(N=\mathbf{2 , 1 5 2})\end{array}$} & \multirow[b]{2}{*}{$t$} & \multirow[b]{2}{*}{$\chi^{2}$} & \multirow[b]{2}{*}{$d$} \\
\hline & $M(S D)$ & $\%(n)$ & $M(S D)$ & $\%(n)$ & & & \\
\hline \multicolumn{8}{|l|}{ Demographics } \\
\hline Age & $33.8(11.4)$ & & $33.1(10.8)$ & & $2.26^{*}$ & & .06 \\
\hline Indigenous & & $15.7(1,386)$ & & $27.6(595)$ & & $165.49 * *$ & .25 \\
\hline Any custody & & $24.5(2,156)$ & & $17.7(380)$ & & $45.48 * *$ & .13 \\
\hline $\mathrm{MH}$ flag ${ }^{1}$ & & $45.0(3,963)$ & & $65.8(1,415)$ & & $306.95 * *$ & .34 \\
\hline \multicolumn{8}{|l|}{ Index Offence $^{2}$} \\
\hline Sexual & & $4.4(387)$ & & $1.3(27)$ & & $46.93 * *$ & .13 \\
\hline Violent & & $43.8(3,855)$ & & $30.6(659)$ & & $123.70 * *$ & .21 \\
\hline Non-violent & & $23.2(2,039)$ & & $37.2(801)$ & & $178.10 * *$ & .26 \\
\hline Admin. & & $4.8(423)$ & & $6.2(133)$ & & $6.79 *$ & .05 \\
\hline Other & & $12.2(1,078)$ & & $12.1(260)$ & & 0.04 & .01 \\
\hline \multicolumn{8}{|l|}{ Full Assessment info ${ }^{3}$} \\
\hline Static risk & $24.8(18.4)$ & & $20.5(15.8)$ & & $10.08 * *$ & & .25 \\
\hline Dynamic risk & $19.2(15.3)$ & & $18.6(15.9)$ & & 1.46 & & .04 \\
\hline Dynamic strength & $27.9(16.4)$ & & $27.1(16.7)$ & & $2.07 *$ & & .05 \\
\hline \multicolumn{8}{|l|}{ Recidivism $^{4}$} \\
\hline Any negative ${ }^{5}$ & & $34.0(2,992)$ & & $30.7(661)$ & & $8.41 *$ & .06 \\
\hline Technical & & $21.1(1,858)$ & & $18.1(390)$ & & $9.50 *$ & .06 \\
\hline New violent & & $15.0(1,317)$ & & $9.8(211)$ & & $38.39 * *$ & .12 \\
\hline Any new & & $26.9(2,370)$ & & $23.4(504)$ & & $11.04 * *$ & .06 \\
\hline
\end{tabular}

Note. $M=$ Mean. $S D=$ Standard deviation. $t=$ t-test. $\chi^{2}=$ Chi square. $d=$ Cohen's d effect size. ${ }^{1} \mathrm{MH}=$ Mental Health. ${ }^{2}$ Non-violent $=$ property, theft, fraud, drug offences, impaired driving. Admin $=$ technical violations and administration of justice offences. Other $=$ traffic and other offences. ${ }^{3}$ Assessment info $=$ based on initial assessment information. ${ }^{4}$ Recidivism $=$ based on 3-year follow-up information. ${ }^{5}$ Any negative outcome $=$ includes any technical violations and/or new charges. Missing data on the index offence was $11.6 \%$ for men and $12.6 \%$ for women. ${ }^{*} p$ $<.05, * * p<.001$ 


\section{Description of domain and total dynamic scores for measurement invariance}

timepoints. Because measurement invariance includes the assessment of how dynamic risk and strength domains contribute to the factor structure of the SPIn, the mean scores and standard deviations, at each of the three timepoints are presented in Table 1D and Table 2D (in Appendix D) for men and women. For both genders, the scores on the dynamic risk domains tended to decrease slightly over the three timepoints, and the scores on the dynamic strength domains tended to increase slightly over the three timepoints.

Assumption checking for exploratory factor analysis. Multicollinearity and singularity were assessed using bivariate correlations (bivariate correlation ranges for dynamic domain scores among each timepoint and for each gender can be found in Tables 3D, 4D, and 5D in Appendix D). Correlations between dynamic domains for men ranged from (+/-) .004 to .703 for Time 1, from (+/-) .001 to .709 for Time 2, and from (+/-) .005 to .711 for Time 3. Correlations between dynamic domains for women ranged from (+/-) .006 to .719 for Time 1 , from (+/-) .006 to .732 for Time 2 , and from (+/-) .022 to .723 for Time 3 . The largest correlation at the domain level was for the relationship between the attitudes dynamic strength domain and skills dynamic strength domain at each timepoint and for both genders, with correlations ranging from .709 to $.732(p<$ .001). The smallest correlation for the men was consistently for the relationship between the employment dynamic strength domain and the family dynamic risk domain across time, with correlations at each timepoint ranging from (+/-) .005 to .012, and the correlation between the family dynamic strength domain and the substance use risk, ranging from (+/-) .001 to .005 at each timepoint. For women, however, the smallest 
correlation changed at each timepoint; at Time 1 (-.006) the smallest correlation was for the relationship between the aggression and violence dynamic strength domain and the employment dynamic risk domain, whereas for Time $2(-.006)$ and Time $3(-.022)$ the smallest correlation was for the relationship between the attitude dynamic strength domain and the family dynamic risk domain. Correlations among Full Assessment total static risk score, total dynamic risk score, and total dynamic strength score did not exceed $.606(p<.001 ;$ see Table 5). 
Table 5

Correlations between SPIn Full Assessment Total Static Risk, Dynamic Risk and Dynamic Strength Score at Each Timepoint

\begin{tabular}{lccc}
\hline & \multicolumn{3}{c}{ Timepoint 1 } \\
\hline \multirow{2}{*}{ Full Assessment } & Full Assessment \\
\cline { 2 - 4 } & Static Risk & Dynamic Risk & Dynamic Strength \\
\hline Static Risk & 1 & 1 & 1 \\
Dynamic Risk & .572 & -.240 & 1 \\
Dynamic Strength & -.078 & Timepoint 2
\end{tabular}

Timepoint 2

\begin{tabular}{lccc}
\hline & \multicolumn{3}{c}{ Full Assessment } \\
\cline { 2 - 4 } Full Assessment & Static Risk & Dynamic Risk & Dynamic Strength \\
\hline Static Risk & 1 & & \\
Dynamic Risk & .592 & 1 & 1 \\
Dynamic Strength & -.077 & -.271 & \\
\hline
\end{tabular}

Timepoint 3

\begin{tabular}{lccc}
\hline & \multicolumn{3}{c}{ Full Assessment } \\
\cline { 2 - 4 } Full Assessment & Static Risk & Dynamic Risk & Dynamic Strength \\
\hline Static Risk & 1 & & \\
Dynamic Risk & .606 & 1 & 1 \\
Dynamic Strength & -.088 & -.285 & 1 \\
\hline
\end{tabular}

Note. All correlations were significant at $p<.001$.

Exploratory Factor Analysis (EFA). To examine measurement invariance, separate EFAs using the 15 dynamic domain scores of the SPIn were conducted at each of the three timepoints. The separate EFA analyses were conducted to better understand the measurement properties of the SPIn over time and whether the same dynamic domain scores contribute to the total dynamic risk score and total dynamic strength score across all three timepoints. 
EFA suitability and factor rotation. MPlus version 8 (Muthén \& Muthén, 19982017) was used to run the EFA and factors were extracted using maximum likelihood method. Results of the bivariate and partial correlations indicate that none of the 15 dynamic domains were highly correlated; the highest correlation was .70 for the men and .72 for the women, although the majority of correlations were under .40 . The Kaiser (KMO) value was .81 for both men and women, which indicates sampling adequacy (Cerny \& Kaiser, 1977). Bartlett's test of sphericity $\left(\chi^{2}(105)=11532.24, p<.001\right)$ was significant indicating that factor analysis is suitable for the data. Upon preliminary inspection, it was determined that orthogonal rotation will be used to run the EFAs across each of the three timepoints.

Results of the EFAs across timepoints. Varimax rotation, a form of orthogonal rotation, was used to conduct EFAs on the 15 dynamic domain scores of the SPIn (Orbis Partners, 2003) at each timepoint, for men and women independently (six EFAs in total). These individual EFA analyses were conducted to examine whether the factor structure (i.e., number and make up of factors) of the SPIn is consistent over time.

As seen in Table 6, three methods were used to determine how many factors to retain: (1) Kaiser's Criterion, where eigenvalues greater than 1.00 are kept, (2) Parallel analysis, which compares eigenvalues of the original dataset to eigenvalues randomly generated by bootstrapping, and (3) Scree plots (Cattell, 1966; O’Connor, 2000; Tabachnick \& Fidell, 2007). Despite mixed findings among the three methods, overall, for each timepoint, a three-factor model was supported and factors were labeled as follows: strength, intrapersonal risk, and interpersonal/stability risk (described in more detail in the next section). 
Table 6

Decision Rules for Factor Retention and Structure Fit at Each Time for Men and Women

\begin{tabular}{lccc}
\hline Timepoint & $\begin{array}{c}\text { Kaiser's } \\
\text { Criterion }\end{array}$ & $\begin{array}{c}\text { Parallel } \\
\text { Analysis }\end{array}$ & $\begin{array}{c}\text { Scree } \\
\text { Plots }\end{array}$ \\
\hline Time $1_{\text {(initial }}$ & & & \\
Men & $3^{\text {a }}$ & 1 & $2-3$ \\
Women & $3^{\text {a }}$ & 1 & $2-3$ \\
Time 2(3-8 months) & & & \\
Men & 3 & 1 & $2-3$ \\
Women & 3 & 1 & $2-3$ \\
Time 3(9-14 months) & & & \\
$\quad$ Men & $3^{\text {a }}$ & 1 & $2-3$ \\
$\quad$ Women & 3 & 1 & $2-3$ \\
\hline
\end{tabular}

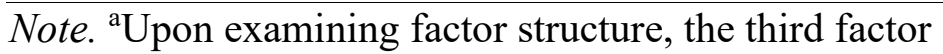
consists of less than 3 factor loadings. As such, only 2 factors emerge with at least 3 factor loadings of .40 or more.

Upon examining factor structure fit of the three-factor model for each timepoint, results were mixed. When looking at the comparative fit index (CFI), the structure fit was found to be good for both men and women across all three timepoints. However, when looking at the Tucker-Lewis index (TLI) and the Rooted Mean Square Error of Approximation (RMSEA), structure fit was found to be acceptable (see Table 7). Notably, one and two factor models were also examined; however, the fit indices indicated poor fit for both. 
Table 7

Model Fit Indices to Examine Factor Structure Fit at Each Time for Men and Women

\begin{tabular}{|c|c|c|c|}
\hline Timepoints & RMSEA [95\% CI] & CFI & TLI \\
\hline \multicolumn{4}{|l|}{ Time $1_{\text {(initial) }}$} \\
\hline Men & $.104[.101, .106]$ & .903 & .831 \\
\hline Women & $.108[.103, .114]$ & .912 & .837 \\
\hline \multicolumn{4}{|l|}{ Time 2 (3-8 months) } \\
\hline Men & $.102[.100, .105]$ & .907 & .837 \\
\hline Women & $.107[.102, .113]$ & .914 & .840 \\
\hline \multicolumn{4}{|c|}{ Time 3 (9-14 months) } \\
\hline Men & $.102[.100, .105]$ & .908 & .839 \\
\hline Women & $.105[.100, .111]$ & .920 & .851 \\
\hline
\end{tabular}

Factor loadings. As seen in Tables 1E and 2E (in Appendix E), loadings are presented for each factor, across each timepoint, for men and women separately. For the men and the women, both the substance use dynamic risk domain and the family dynamic risk domain did not load onto any factor across all timepoints (factor loadings less than .40), and as such, were removed from the final factor structure. The three factor-structure that emerged for men was the same three factor-structure that emerged for women. Specifically, Factor 1 is defined by strength factors (made up of all 7 dynamic strength domains). Factor 2 is defined by intrapersonal risk factors (made up of 3 dynamic risk domains; aggression, attitudes and skills). Factor 3 is defined by interpersonal stability risk factors (made up of 3 dynamic risk domains; stability, social influences, and employment). The internal consistency of the three factors are presented in Table 3E, and 
ranged from acceptable to good across the three timepoints for men and women for each of the factors.

Exploratory Structural Equation Modeling (ESEM). ESEM was used to examine the extent to which the factor loadings and factor structure obtained previously during the EFAs above are invariant over time within each gender across different measurement invariance models (with different parameter constraints).

Measurement invariance models to be tested. Two types of measurement invariance models were tested: the configural model and the scalar model. The configural model is a baseline model used to compare to more restrictive models. It includes no parameter constraints and tests whether the constructs have the same pattern of free and fixed loadings across timepoints (Putnick \& Bornstein, 2016). The scalar invariance model is a more restrictive model that assesses whether factor loadings and intercepts are equivalent across timepoints (Putnick \& Bornstein, 2016). Essentially, achieving scalar measurement invariance provides evidence to support the utility of mean comparisons across time, indicating that the same underlying construct is being measured at each timepoint.

Similar to assessing factor structure fit in the context of EFA, model fit of the configural invariance model and the scalar invariance model was assessed using RMSEA, CFI, and TLI fit indices. Notably, measurement invariance was assessed independently for men and women as all further analyses are also ran by each gender independently.

Measurement invariance results for the dynamic strength factor. As Table 8 indicates, for men there was an improvement in model fit when comparing the scalar to the configural model $\left(\mathrm{CFI}_{\text {change }}=.001, \mathrm{TLI}_{\text {change }}=.002, \mathrm{RMSEA}_{\text {change }}=.013\right)$. For the 
women (see Table 9), there was no change in the CFI between the scalar and configural models. However, the other fit indices indicated slight improvement when comparing the scalar to the configural model $\left(\mathrm{TLI}_{\text {change }}=.001, \mathrm{RMSEA}_{\text {change }}=.003\right)$, indicating that the scalar model fit the data better. In other words, for both men and women, the dynamic strength factor met the assumption of measurement invariance.

\section{Measurement invariance results for the dynamic intrapersonal risk factor. As}

Table 8 indicates, for men there was no improvement in model fit when comparing the CFI and TLI for scalar and configural models due to the fact that both demonstrated maximum model fit $(\mathrm{CFI}=1.00, \mathrm{TLI}=1.00)$. However, upon examining the change in RMSEA between models, it was found that the configural model had better fit than the scalar model $\left(\mathrm{RMSEA}_{\text {configural }}=.011\right.$ vs. RMSEA $\left._{\text {scalar }}=.013\right)$. Similarly, for the women (see Table 9), there was no improvement in model fit when comparing the CFI and TLI for scalar and configural models due to the fact that both demonstrated maximum model fit $(\mathrm{CFI}=1.00, \mathrm{TLI}=.999)$. However, when comparing the scalar to the configural model, the change in RMSEA was .008, indicating that the scalar model fit the data better. In other words, for women, the dynamic intrapersonal risk factor met the assumption of measurement invariance, however, for men, this assumption was not met.

Measurement invariance results for the interpersonal / stability risk factor. As Table 8 indicates, for men there was no improvement in model fit when comparing the CFI and TLI for scalar and configural models due to the fact that both demonstrated maximum model fit $(\mathrm{CFI}=1.00, \mathrm{TLI}=1.00)$. However, upon examining the change in RMSEA between models, it was found that the configural model had better fit than the scalar model $\left(\right.$ RMSEA $_{\text {configural }}=.005$ vs. RMSEA $\left._{\text {scalar }}=.012\right)$. Similarly, for the women 
(see Table 9), there was no improvement in model fit when comparing the CFI and TLI for scalar and configural models due to the fact that both demonstrated maximum model fit $(\mathrm{CFI}=1.00, \mathrm{TLI}=1.00)$. However, when comparing the scalar to the configural model, the change in RMSEA was .008 , indicating that the scalar model fit the data better. In other words, for women, the dynamic interpersonal/stability risk factor met the assumption of measurement invariance, however, for men, this assumption was not met.

Table 8

Model Fit Indices and Nested Model Comparisons for Testing Configural and Scalar Measurement Invariance for Men

\begin{tabular}{lccc}
\hline Factor 1: Strength & CFI & TLI & RMSEA [95\% CI $]$ \\
\hline Configural $^{1}$ & .995 & .994 & $.080[.078, .081]$ \\
Scalar $^{2}$ & .996 & .996 & $.067[.065, .070]$ \\
\hline Factor 2: Intrapersonal Risk $^{2}$ & CFI & TLI & RMSEA [95\% CI] \\
\hline Configural & 1.000 & 1.000 & $.011[.005, .016]$ \\
Scalar & 1.000 & 1.000 & $.013[.009, .017]$ \\
\hline Factor 3: Interpersonal/Stability Risk & CFI & TLI & RMSEA [95\% CI $]$ \\
\hline Configural & 1.000 & 1.000 & $.005[.000, .012]$ \\
Scalar & 1.000 & 1.000 & $.012[.008, .015]$ \\
\hline
\end{tabular}

Note. CFI = Comparative Fit Index. TLI $=$ Tucker-Lewis Index. RMSEA = Rooted Mean Square Error of Approximation. $\mathrm{CI}=$ Confidence Interval. ${ }^{1}$ Configural $=$ No equality constraints. ${ }^{2}$ Scalar $=$ Factor loadings and intercepts constrained to be equal across all time. 
Table 9

Model Fit Indices and Nested Model Comparisons for Testing Configural and Scalar Measurement Invariance for Women

\begin{tabular}{lccc}
\hline Factor 1: Strength & CFI & TLI & RMSEA [95\% CI] \\
\hline Configural $^{1}$ & .997 & .996 & $.056[.053, .059]$ \\
Scalar $^{2}$ & .997 & .997 & $.053[.050, .055]$ \\
\hline Factor 2: Intrapersonal Risk & CFI & TLI & RMSEA [95\% CI] \\
\hline Configural & 1.000 & .999 & $.039[.029, .049]$ \\
Scalar & 1.000 & .999 & $.031[.023, .038]$ \\
\hline Factor 3: Interpersonal/Stability Risk & CFI & TLI & RMSEA [95\% CI $]$ \\
\hline Configural & 1.000 & 1.000 & $.027[.016, .037]$ \\
Scalar & 1.000 & 1.000 & $.019[.010, .027]$ \\
\hline
\end{tabular}

Note. CFI = Comparative Fit Index. TLI $=$ Tucker-Lewis Index. RMSEA $=$ Rooted Mean Square Error of Approximation. CI $=$ Confidence Interval. ${ }^{1}$ Configural $=$ No equality constraints. ${ }^{2}$ Scalar $=$ Factor loadings and intercepts constrained to be equal across time.

In practice, the SPIn dynamic domains are split into two constructs (total dynamic strength and total dynamic risk). As such measurement invariance of this two-factor model was also assessed. Overall, the two-factor model was found to be invariant over time (see Appendix F); in fact, the results demonstrated stronger evidence of measurement invariance than the three-factor model. As such, the two-factor model, total dynamic strength and total dynamic risk, will be used in the subsequent change analyses as opposed to the three-factors that emerged in the initial EFA 9 .

\footnotetext{
${ }^{9}$ The EFA conducted in the measurement invariance section was the first to be assessed on the SPIn. Unfortunately, due to lack of SPIn item-level data over time, an EFA could not be conducted using the 90item full SPIn assessment. As such, the EFA was conducted using the 15 SPIn dynamic domain scores, and because the internal consistency of several of the domains was low (see Appendix B, Table 1B), using domain scores to assess factor structure is not ideal. Additionally, the three-factor structure that emerged excluded the substance use dynamic risk domain and the family dynamic risk domain, as they did not load onto either of the three emerging factors. Overall, as these three factors are not representative of how full SPIn assessments are used operationally, the two-factor structure used in practice (which was found to meet the assumption of measurement invariance) was used instead for all subsequent analyses.
} 


\section{Patterns of change: Description of Analyses}

The current study used hierarchical linear growth modeling (HLGM) to examine patterns of change at the offender level (for men and women separately) and the extent to which static risk score, age, and Indigenous status are related to different patterns of change.

Hierarchical linear growth modeling. HLGM is designed to examine data clustered into groups whereby patterns of change within individuals and change between individuals can both be examined (Hox, 2010; Singer \& Willett, 2003). This approach explores average growth trends while providing an understanding of the types of variables that explain variability in the observed growth trends. There are several advantages to using HLGM over other approaches. First, HLGM can analyze non-normal data and can model time-unstructured longitudinal designs whereby assessments are unbalanced and take place at different intervals of time, even if all assessments are not available for all participants (Hox, 2010; Singer \& Willett, 2003). Additionally, HLGM analyses have few parametric assumptions (Garson, 2013; Hox, 2010). For instance, HLGM does not require the assumption of sphericity, also referred to as having equal variance and covariance across timepoints (Tabachnick \& Fidell, 2007), and allows the measurement of heteroscedastic models which cannot be estimated by other approaches (Raudenbush \& Bryk, 2002). HLGM can also model random effects, which allows for the consideration of unmeasured heterogeneity (Babchishin, 2013). It is important to note, however, that HLGM puts more weight on data that comes from participants with more assessments over longer time periods, within the main time frame of the study (Lloyd, 2015). 
There are some limitations to using HLGM. For instance, HLGM cannot incorporate and examine two simultaneous change processes, such as changes in dynamic risk score and the simultaneous changes in dynamic strength score (Lloyd, 2015). In addition, HLGM also assumes that the missing data are missing at random, which can cause issues for re-entry datasets (Lloyd, 2015). Nonetheless, HLGM with correctional datasets have been used in several recent studies (e.g., Babchishin, 2013; Hanby, 2013; Orrick, 2012). As such, the current study is running an HLGM using unbalanced and time-unstructured data to examine: 1) the extent to which the total dynamic risk score and total dynamic strength score from the SPIn Full Assessment change over time, 2) the heterogeneity in the pattern of change across offenders, and 3) the extent to which total static risk score from the full SPIn assessment, Indigenous status, and age are related to the different patterns of change. These analyses are conducted for men and women independently.

Model building process. Independently for women and men, three models were created using the SPIn Full Assessment total dynamic risk score as an outcome variable: the unconditional means model, the unconditional growth model, and the conditional growth model. Similarly, for both women and men, the unconditional means model, unconditional growth model, and the conditional growth model were also created using the SPIn Full Assessment total strength score as an outcome variable. The unconditional means model simply describes the variation in the initial total dynamic risk scores or total dynamic strength scores and does not consider change. The unconditional growth model includes time as the only predictor in the model to examine within-individual effects (e.g., level-1). Finally, the conditional model, includes time, as well as level-2 predictors, 
to examine both within- and between-individual effects. ${ }^{10}$ These level-2 predictors include age, Indigenous status, and SPIn Full Assessment static risk scores, which may help to explain differences in initial total dynamic risk or total dynamic strength scores (i.e., intercept) as well as change in these scores over time (i.e., slopes). ${ }^{11}$

Fit statistics and assumptions. To assess goodness of model fit for each of the three models, multiparameter tests such as AIC and BIC statistics were used, as well as -2 log likelihood (-2LL) values, ideal for nested models (Bell, Ene, Smiley, \& Schoeneberger, 2013; Singer \& Willett, 2003). Overall, the model with the lowest AIC, $\mathrm{BIC}$, and -2LL is preferred for the data.

Time. Time can be entered into the model as either a linear, quadratic, or cubic trend. To determine the best way to enter time into the model, the loglikelihood ratio test was used to assess which trend best fit the data. The unconditional growth model was used to examine the shape of each offender's trajectory of change over time. In addition to the different trends, time can also be operationalized in a number of ways, which influences how the findings are interpreted (Babchishin, 2013; Blozis \& Cho, 2008). In the current study, timepoints were centered around the initial assessment date, in months, to allow for ease of interpretation (i.e., initial assessment is considered timepoint 0 , each subsequent month that passes are considered a new timepoint). ${ }^{12}$

\footnotetext{
${ }^{10}$ See Raudenbush and Bryk (2002) for notations and equations specific to the HLGM analyses for longitudinal data.

${ }^{11}$ Since the total number of assessments could only range between 3 and 5, the total number of assessments was not included as a potential control variable, neither was follow-up time, as the current sample all had a three-year fixed follow-up.

12 The relative standard deviation was calculated, which is the square root of the variance of timepoints divided by the mean timepoints in sample, and was found to be $3.67 \%$ (square root of 51.71/3.83), thus the type of centering technique used would not influence the parameters produced (Blozis \& Cho, 2008).
} 
Extra assumptions specific to HLGM. Most assumptions were tested at the first level (i.e., level-1) of the model, which was reported in the statistical and methodological issues section; however, assumptions including linearity and homoscedasticity were also assessed at level-2. For men, based on various plots (i.e., histograms) and the skewness coefficient of the total dynamic risk score and total dynamic strength score (1.27 and .66, respectively), skewness was not an issue, indicating no violations of normality and no need for transformations of the data. For women, the skewness coefficient for the total dynamic risk score (1.52) and the total dynamic strength score (.87), skewness was also not an issue, indicating no violations of normality. However, upon examining the various plots, there was a slight skew in normality for the total dynamic strength scores. Given that there was only a slight skew, and to maintain clear interpretation of the findings, no transformations were made to this variable (Feng et al., 2014). Additionally, given that the goal is to examine differences in rates of change, a level of skewness in data is expected. Residuals for the random effects were examined using Q-Q plots and appear to be relatively normal for both men and women for the total dynamic risk and strength scores, with less variability over time and some deviation in the tail ends.

Another assumption to examine is shrinkage. Shrinkage in regression models refers to the bias found between sample statistics and inferred population parameters, which can occur in multiple regression. Notably, because the sample size is large and the number of covariates is small, shrinkage is not a problem for the current study (Copas, 1997; Steyerberg, Eijkemans, Harrell, \& Habbema, 2001).

Finally, a sensitivity analysis was conducted to determine whether outliers (defined as extreme standardized residuals in the 0 and $99^{\text {th }}$ percentile) are influencing 
overall results. For the men, nine outliers were identified, and for the sample of women, only one outlier was identified upon running the sensitivity analyses. Similar results were obtained when the potential outliers were removed for both the men $(N=11,944$ and 43,079 observations) and women ( $N=2,876$ and 10,145 observations). As such, final results will be presented with all valid cases included.

\section{Patterns of Change Results}

To assess the HLGM pattern of change results, the first step is to determine the best trend that fit the time data; that is, determining whether time is best defined by a linear, quadratic, or cubic trend. Next the results from the unconditional means model are used to examine the proportion of variance in initial total dynamic risk and total dynamic strength scores that exists between individuals. This is followed by change over time results presented through the unconditional growth model, as well as the effects of level-2 predictors (age, Indigenous status, and total static risk score) assessed in the conditional growth model. Notably, the proportion of explained variability associated with each model is also presented.

Patterns of change results: Women and total dynamic risk scores. The initial assessment SPIn total dynamic risk scores ranged from 0 to $163(M=18.73, S D=15.89)$. Subsequent SPIn assessments occurred at intervals spanning 3 to 30 months post initial assessment and the total dynamic risk scores from these subsequent assessments ranged from 0 to $147(M=18.77, S D=15.39)$.

Trend of time data. Overall it was found that a random effects linear function fit the time data better than a quadratic function. Specifically, the loglikelihood value associated with the random effects linear function (73447.3) was smaller than the 
loglikelihood value associated with the quadratic function (74983.7), and as such, the likelihood ratio test could not be ran, indicating that the linear random effects trend best fit the data. ${ }^{13}$ This indicates that the total dynamic risk scores steadily increase or decrease over time.

Unconditional means model: Variance between individuals. To examine the necessity of using multilevel models, the unconditional means model is used to calculate the intraclass correlation. The intraclass correlation coefficient (ICC) was calculated by dividing the intercept variance by the total variance (intercept and residual variance) $)^{14}$, see model results presented in Table 10. The ICC was .852 $(201.93 /(35.01+201.93))$, which indicates that $85.2 \%$ of the variance in initial total dynamic risk scores can be explained by differences among justice-involved women.

Unconditional growth model: Level-1 change over time. As seen in Table 10, results showed that, on average the initial total dynamic risk score for women was 18.54 and the average rate of change was a decrease in scores by .04 points per month while on community supervision. The variance in initial status $\left(\tau_{00}=231.54\right)$ and rate of change $\left(\tau_{11}=.29\right)$ were both significantly different from zero $(p<.001)$, indicating that there was significant variability in both initial total dynamic risk scores and in the rate of change on these scores among women. Further, the covariance between initial score and change over time $\left(\tau_{01}=-2.62, p<.001\right)$ suggests that women with higher total dynamic risk scores at initial assessment evidenced a greater decrease in scores over time relative to women with lower total dynamic risk scores at initial assessment.

\footnotetext{
${ }^{13}$ Given that the linear function fit the data better than the quadratic function, the cubic function was not examined.

${ }^{14} \mathrm{ICC}=\tau_{00} /\left(\tau_{00}+\sigma^{2}\right)$
} 
Conditional growth model: Variation as a function of level-2 predictors. The conditional growth model included three level-2 predictors: age, Indigenous status, and total static risk scores. As seen in Table 10, initial total dynamic risk scores did not vary as a function of age or Indigenous status, however, scores did vary as a function of total static risk score $\left(\gamma_{03}=.64, S E=.01, p<.001\right)$. Similarly, change in total dynamic risk scores did not vary as a function of age and Indigenous status, however, these scores did change over time as a function of total static risk scores $\left(\gamma_{13}=-.01, S E=.00, p<.001\right)$; although the magnitude of this effect was extremely small. 
Table 10

Two-level Linear Growth Models Examining Changes in Total Dynamic Risk Scores Over Time for Women $(N=2,877)$

\begin{tabular}{|c|c|c|c|c|c|c|c|}
\hline & & \multicolumn{2}{|c|}{$\begin{array}{c}\text { Unconditional } \\
\text { Means }\end{array}$} & \multicolumn{2}{|c|}{$\begin{array}{c}\text { Unconditional } \\
\text { Growth }\end{array}$} & \multicolumn{2}{|c|}{$\begin{array}{l}\text { Conditional } \\
\text { Growth }\end{array}$} \\
\hline & & Estimate & $(S E)$ & Estimate & $(S E)$ & Estimate & $(S E)$ \\
\hline \multicolumn{8}{|c|}{ Fixed Effects Initial Status } \\
\hline & Intercept $\left(\gamma_{00}\right)$ & $18.30 * * *$ & $(.27)$ & $18.54 * * *$ & $(.29)$ & $19.20 * * *$ & $(.29)$ \\
\hline & Age $\left(\gamma_{01}\right)$ & -- & -- & -- & -- & .02 & $(.02)$ \\
\hline & Indigenous $\left(\gamma_{02}\right)$ & -- & -- & -- & -- & .88 & $(.54)$ \\
\hline & Static Risk $\left(\gamma_{03}\right)$ & -- & -- & -- & -- & $.64 * * *$ & $(.01)$ \\
\hline \multicolumn{8}{|c|}{ Rate of Change } \\
\hline & Intercept $(\gamma 10)$ & -- & -- & $-.04 * *$ & $(.01)$ & $-.11 * * *$ & $(.01)$ \\
\hline & Time*Age $(\gamma 11)$ & -- & -- & -- & -- & .00 & $(.00)$ \\
\hline & Time*Indigenous $(\gamma 12)$ & -- & -- & -- & -- & .03 & $(.03)$ \\
\hline & Time*Static Risk $(\gamma 13)$ & -- & -- & -- & -- & $-.01 * * *$ & $(.00)$ \\
\hline \multicolumn{8}{|c|}{ Variance Components } \\
\hline Level 1 & Within Person $\left(\sigma^{2}\right)$ & $35.01 * * *$ & $(.57)$ & $18.93 * * *$ & $(.38)$ & $16.69 * * *$ & $(.33)$ \\
\hline \multirow[t]{3}{*}{ Level 2} & Initial Status $\left(\tau_{00}\right)$ & $201.93 * * *$ & $(5.59)$ & $231.54 * * *$ & $(6.46)$ & $150.76^{* * *}$ & $(4.31)$ \\
\hline & Rate of change $\left(\tau_{11}\right)$ & -- & -- & $.29 * * *$ & $(.01)$ & $.22 * * *$ & $(.01)$ \\
\hline & Covariance $\left(\tau_{01}\right)$ & -- & -- & $-2.62 * * *$ & $(.21)$ & $-2.51 * * *$ & $(.16)$ \\
\hline \multicolumn{8}{|l|}{ Fit Indices } \\
\hline & AIC & \multicolumn{2}{|c|}{74989.9} & \multicolumn{2}{|c|}{73269.2} & \multicolumn{2}{|c|}{70707.6} \\
\hline & $\mathrm{BIC}$ & \multicolumn{2}{|c|}{75007.8} & \multicolumn{2}{|c|}{73304.9} & \multicolumn{2}{|c|}{70779.1} \\
\hline & -2 Loglikelihood & \multicolumn{2}{|c|}{74983.9} & \multicolumn{2}{|c|}{73257.2} & \multicolumn{2}{|c|}{70683.6} \\
\hline
\end{tabular}

Note. Unconditional growth model assesses within-individual effects (level-1). Conditional growth model includes between-individual effects (level-2 predictors). Estimate $=$ regression coefficient. $S E=$ Standard Error. AIC $=$ Akaike Information Criterion. BIC $=$ Bayesian Information Criterion. Estimation Method = Maximum Likelihood; Satterthwaite degrees of freedom. ${ }^{* * *} p<.001,{ }^{*} p<.01,{ }^{*} p<.05$ 
Figure 4 highlights the differences in initial total dynamic risk scores and changes in total dynamic risk scores as a function of total static risk scores. Specifically, women with an average static risk score of 19.2 evidenced an average decrease in total dynamic risk scores of .11 points per month. In comparison, women with a total static risk score one standard deviation below the mean (score of 8.51) evidenced an average increase in total dynamic risk scores of .06 points per month. Finally, women with a total static risk score one standard deviation above the mean (score of 29.89) evidenced an average decrease in total dynamic risk scores of .28 points per month.

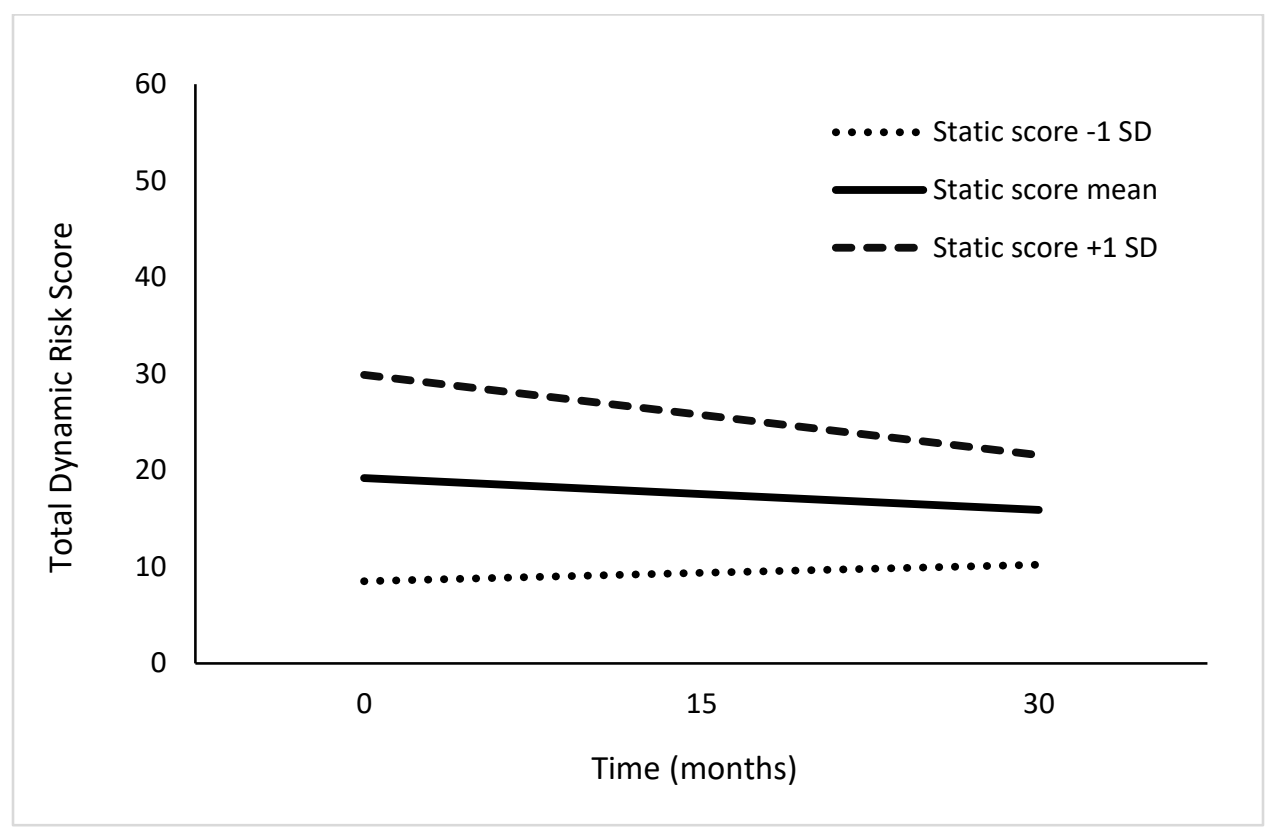

Figure 4. Women's change in total dynamic risk scores over time as a function of total static risk score.

Figure 5 presents the initial total dynamic risk scores and the change in total dynamic risk scores over 30 months for a random sample of 500 justice-involved women $(17.4 \%$ of the sample). 


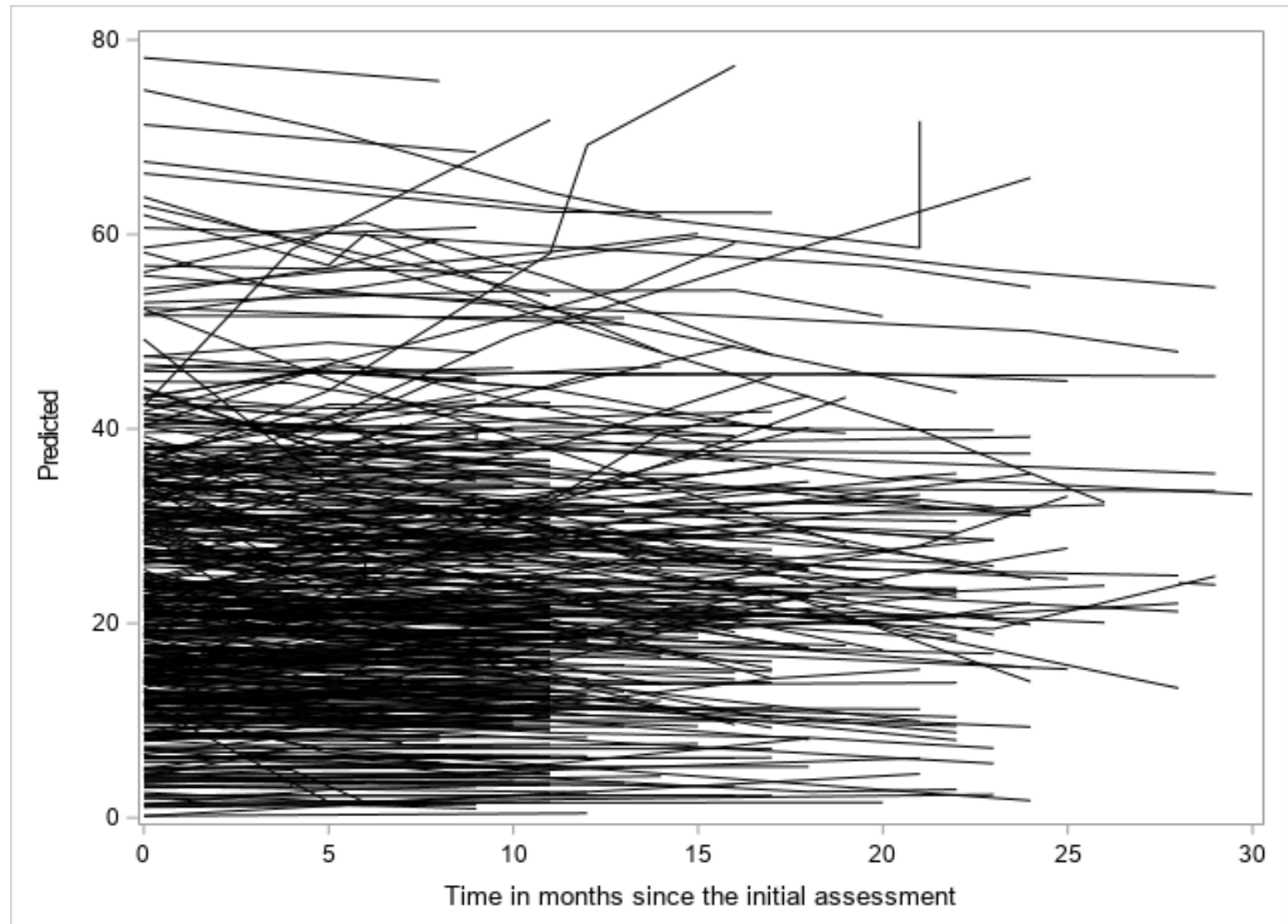

Figure 5. Individual change on total dynamic risk scores (predicted) across time in months for women.

Pseudo $\boldsymbol{R}^{2}$ results. As described in Table 11, pseudo $R^{2}$ statistics are calculated to quantify the amount of variation explained by the growth models. Pseudo $R^{2}$ for total outcome provides the proportion of total variation in total dynamic risk scores explained by the growth models. For instance, less than $1 \%$ of variance is explained by the unconditional growth model, whereas $36 \%$ of variance is explained by the conditional growth model. Pseudo $R^{2}$ statistics are also calculated to quantify the proportion of within-person variation explained by time. For the unconditional growth model, the proportion of within-offender variation explained by time is $45.9 \%$, whereas for the conditional growth model, the proportion of within-individual variation explained by 
time is $52.3 \%$. Finally, pseudo $R^{2}$ statistics are also calculated to examine the proportional reduction in level-2 residual variance at the intercept and slope, with the addition of various predictor variables in the conditional growth model. That is, adding level-2 predictors was found to further explain unexplained outcome variation for both the initial total dynamic risk scores (34.9\%) and change in total dynamic risk scores over time $(24.1 \%)$.

Table 11

Comparison of Pseudo $R^{2}$ Statistics between Unconditional and Conditional Growth Models for Women-Total Dynamic Risk Scores

\begin{tabular}{lcc}
\hline Pseudo $\boldsymbol{R}^{2}$ & $\begin{array}{c}\text { Unconditional } \\
\text { Growth }\end{array}$ & $\begin{array}{c}\text { Conditional } \\
\text { Growth }\end{array}$ \\
\hline Total Outcome & .003 & .360 \\
Within-person residual variance & .459 & .523 \\
Intercept & -- & .349 \\
Slope & -- & .241 \\
\hline
\end{tabular}

Note. Unconditional growth only contains (within-person) level-1 predictors. Conditional growth model includes (between-person) level-2 predictors.

In sum, it is evident that total dynamic risk scores overall tend to steadily decrease for women over time; however, this rate of change is quite gradual and minimal. Importantly, Indigenous status and age did not influence the initial total dynamic risk score or the change in total dynamic risk scores over time. Women's total static risk scores, however, did influence both their initial total dynamic risk score and change in total dynamic risk scores over time. Specifically, women with higher initial static risk scores had higher total dynamic risk scores and these scores tended to decrease at faster rates over time, than women who had lower static risk scores. Overall, the conditional 
growth model which included total static risk score explained a significant amount of variability in initial scores and change in scores over time.

Patterns of change results: Women and total dynamic strength scores. The initial assessment SPIn total dynamic strength scores ranged from 0 to $87(M=26.84, S D$ $=16.51)$. Subsequent SPIn assessments occurred at intervals spanning 3 to 30 months post initial assessment and the total dynamic strength scores from these subsequent assessments ranged from 0 to $89(M=27.35, S D=16.32)$.

Trend of time data. Overall it was found that a random effects linear function fit the time data better than a quadratic function. The loglikelihood value associated with the random effects linear function (70578.3) was smaller than the loglikelihood value associated with the quadratic function (72423.4), and as such, the likelihood ratio test could not be ran, indicating that the linear random effects trend best fit the data. ${ }^{15}$ This indicates that the total dynamic strength scores steadily increase or decrease over time.

Unconditional means model: Variance between individuals. The intraclass correlation coefficient (ICC) was calculated and was found to be $.912(246.03 /(23.72+$ 246.03)), which indicates that $91.2 \%$ of the variance in initial total dynamic strength scores can be explained by differences among justice-involved women (see Table 12).

Unconditional growth model: Level-1 change over time. As seen in Table 12, results indicated that, on average, the initial total dynamic strength score for women was 26.56 and the average rate of change was an increase in scores by .08 points per month while on community supervision. The variance in initial status $\left(\tau_{00}=256.58\right)$ and rate of change $\left(\tau_{11}=.20\right)$ were both significantly different from zero $(p<.001)$, indicating that

\footnotetext{
${ }^{15}$ Given that the linear function fit the data better than the quadratic function, the cubic function was not examined.
} 
there was significant variability in both initial total dynamic strength scores and in the rate of change on these scores among women. Further, the covariance between initial score and change over time $\left(\tau_{01}=-1.28, p<.001\right)$ suggests that women with lower total dynamic strength scores at initial assessment evidenced a greater increase in scores over time relative to women with higher total dynamic strength scores at initial assessment.

Conditional model: Variation as a function of level-2 predictors. The conditional model included three level-2 predictors: age, Indigenous status, and total static risk scores. As seen in Table 12, initial total dynamic strength scores did not vary as a function of Indigenous status, however, scores did vary as a function of age $\left(\gamma_{01}=.06\right.$, $S E=.03, p<.05)$, whereby those who were younger had lower initial dynamic strength scores than those who were older. Initial total dynamic strength scores also varied as a function of total static risk score $\left(\gamma_{03}=-.16, S E=.01, p<.001\right)$, where those who had higher total static risk scores had lower initial total dynamic strength scores relative to those who had lower total static risk scores. Change in total dynamic strength scores, however, was found to vary as a function of age $\left(\gamma_{11}=-.003, S E=.001, p<.05\right)$, Indigenous status $\left(\gamma_{12}=-.06, S E=.02, p<.01\right)$, and total static risk scores $\left(\gamma_{13}=.002, S E\right.$ $=.001, p<.01)$. However, the magnitude of these effects was extremely small. 
Table 12

Two-level Linear Growth Models Examining Changes in Total Dynamic Strength Scores Over Time for Women $(N=2,877)$

\begin{tabular}{|c|c|c|c|c|c|c|c|}
\hline & \multicolumn{2}{|c|}{$\begin{array}{c}\text { Unconditional } \\
\text { Means }\end{array}$} & \multicolumn{2}{|c|}{$\begin{array}{c}\text { Unconditional } \\
\text { Growth }\end{array}$} & \multicolumn{2}{|c|}{$\begin{array}{c}\text { Conditional } \\
\text { Growth }\end{array}$} \\
\hline & & Estimate & $(S E)$ & Estimate & $(S E)$ & Estimate & $(S E)$ \\
\hline \multicolumn{8}{|c|}{ Fixed Effects Initial Status } \\
\hline & Intercept $\left(\gamma_{00}\right)$ & $27.16^{* * *}$ & $(.30)$ & $26.56 * * *$ & $(.30)$ & $26.29 * * *$ & $(.37)$ \\
\hline & Age $\left(\gamma_{01}\right)$ & -- & -- & -- & -- & $.06^{*}$ & $(.03)$ \\
\hline & Indigenous $\left(\gamma_{02}\right)$ & -- & -- & -- & -- & .14 & $(.69)$ \\
\hline & Static Risk $\left(\gamma_{03}\right)$ & -- & -- & -- & -- & $-.16 * * *$ & $(.01)$ \\
\hline \multicolumn{8}{|c|}{ Rate of Change } \\
\hline & Intercept $(\gamma 10)$ & -- & -- & $.08 * * *$ & $(.01)$ & $.11 * * *$ & $(.01)$ \\
\hline & Time*Age $(\gamma 11)$ & -- & -- & -- & -- & $-.003 *$ & $(.00)$ \\
\hline & Time*Indigenous $(\gamma 12)$ & -- & -- & -- & -- & $-.06 * *$ & $(.02)$ \\
\hline & Time*Static Risk $(\gamma 13)$ & -- & -- & -- & -- & $.002 * *$ & $(.00)$ \\
\hline \multicolumn{8}{|c|}{ Variance Components } \\
\hline Level 1 & Within Person $\left(\sigma^{2}\right)$ & $23.72 * * *$ & $(.39)$ & $12.05 * * *$ & $(.24)$ & $11.80 * * *$ & $(.24)$ \\
\hline \multirow[t]{3}{*}{ Level 2} & Initial Status $\left(\tau_{00}\right)$ & $246.03 * * *$ & $(6.67)$ & $256.58 * * *$ & (6.99) & $260.01 * * *$ & $(7.14)$ \\
\hline & Rate of change $\left(\tau_{11}\right)$ & -- & -- & $.20 * * *$ & $(.01)$ & $.20 * * *$ & $(.01)$ \\
\hline & Covariance $\left(\tau_{01}\right)$ & -- & -- & $-1.28 * * *$ & $(.18)$ & $-1.37 * * *$ & $(.18)$ \\
\hline \multicolumn{8}{|l|}{ Fit Indices } \\
\hline & AIC & \multicolumn{2}{|c|}{72585.1} & \multicolumn{2}{|c|}{70530.0} & \multicolumn{2}{|c|}{70382.5} \\
\hline & $\mathrm{BIC}$ & \multicolumn{2}{|c|}{72603.0} & \multicolumn{2}{|c|}{70565.8} & \multicolumn{2}{|c|}{70454.0} \\
\hline & -2 Loglikelihood & \multicolumn{2}{|c|}{72579.1} & \multicolumn{2}{|c|}{70518.0} & \multicolumn{2}{|c|}{70358.5} \\
\hline
\end{tabular}

Note. Unconditional growth model assesses within-individual effects (level-1). Conditional growth model includes between-individual effects (level-2 predictors). Estimate $=$ regression coefficient. $S E=$ Standard Error. AIC $=$ Akaike Information Criterion. BIC $=$ Bayesian Information Criterion. Estimation Method = Maximum Likelihood; Satterthwaite degrees of freedom. ${ }^{* *} p<.001,{ }^{* *} p<.01,{ }^{*} p<.05$ 
Although changes in total dynamic strengths scores were found to vary as a function of age and total static risk scores, the magnitude of these effects was extremely small (less than .01), and as such, will not be presented in a figure. However, Figure 6 highlights the differences in changes on total dynamic strength scores over time as a function of Indigenous status. Specifically, Indigenous women had an average initial dynamic strength score of 26.16 and evidenced an average increase in total dynamic strength scores of .08 points per month. In comparison, non-Indigenous women had an average initial dynamic strength score of 26.42 and evidenced an average increase in total dynamic strength scores of .14 points per month.

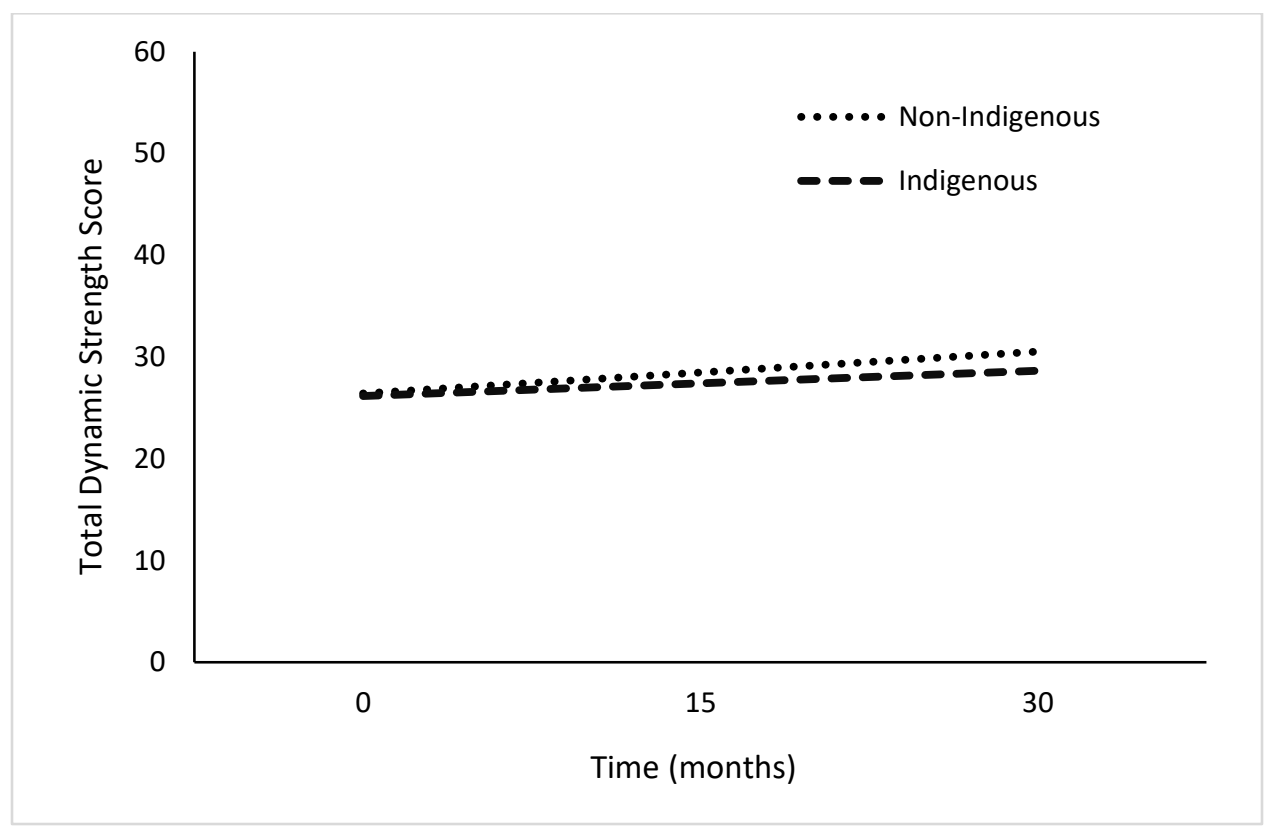

Figure 6. Women's change in total dynamic strength scores over time as a function of Indigenous status.

Figure 7 presents the initial total dynamic strength scores and the change in total dynamic strength scores over 30 months for a random sample of 500 justice-involved women $(17.4 \%$ of the sample). 


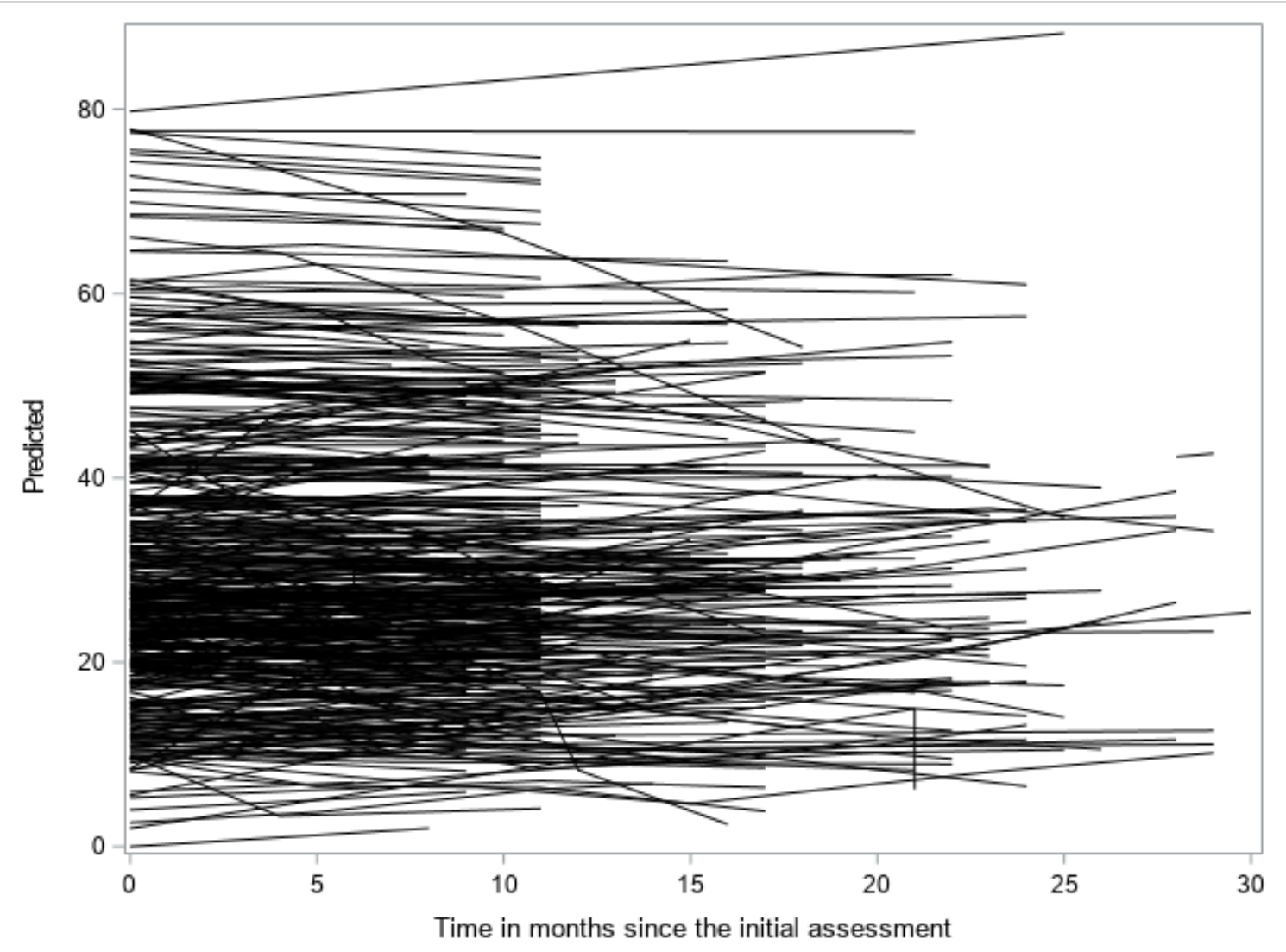

Figure 7. Individual change on total dynamic strength scores (predicted) across time in months for women.

Pseudo $\boldsymbol{R}^{2}$ results. As described in Table 13, pseudo $R^{2}$ statistics are calculated to quantify the amount of variation explained by the growth models. Results indicated that less than $1 \%$ of variance is explained by the unconditional growth model, however, the conditional growth model did not explain any additional variance (less than $1 \%$ of variance remains explained by the conditional growth model). For the unconditional growth model, the proportion of within-offender variation explained by time is $49.2 \%$, whereas for the conditional growth model, the proportion of within-individual variation explained by time is $50.3 \%$. Finally, adding level-2 predictors was not found to further 
explain unexplained outcome variation for both the initial total dynamic strength scores $(1.3 \%)$ and the total dynamic strength scores over time (less than $1 \%$ ).

Table 13

Comparison of Pseudo $R^{2}$ Statistics between Unconditional and Conditional Growth Models for Women-Total Dynamic Strength Scores

\begin{tabular}{ccc}
\hline Pseudo $\boldsymbol{R}^{2}$ & $\begin{array}{c}\text { Unconditional } \\
\text { Growth }\end{array}$ & $\begin{array}{c}\text { Conditional } \\
\text { Growth }\end{array}$ \\
\hline Total Outcome & .001 & .005 \\
Within-person residual variance & .492 & .503 \\
Intercept & -- & .010 \\
Slope & -- & .001 \\
\hline
\end{tabular}

Note. Unconditional growth only contains (within-person) level-1 predictors. Conditional growth model includes (between-person) level-2 predictors.

In sum, it is evident that total dynamic strength scores overall tend to steadily increase for women over time, however, this rate of change is quite gradual and minimal. Importantly, Indigenous status was not found to influence initial total dynamic strength score. Age and total static risk score were both found to influence the initial total dynamic strength score, whereby women who were younger as well as those who had higher total static risk scores, tended to have lower initial dynamic strength scores. Women's age, Indigenous status, and total static risk scores were found to influence change in total dynamic strength scores over time, although the influence of age and static risk was quite minimal. Notably, while Indigenous and non-Indigenous women had similar initial dynamic strength scores, these scores increased at faster rates over time for non-Indigenous women. Overall, the conditional model did not explain a significant amount of variability in initial scores and change in scores over time. 
Patterns of change results: Men and total dynamic risk scores. The initial assessment SPIn total dynamic risk scores ranged from 0 to $225(M=19.32, S D=15.83)$. Subsequent SPIn assessments occurred at intervals spanning 3 to 30 months post initial assessment and the total dynamic risk scores from these subsequent assessments ranged from 0 to $176(M=19.04, S D=15.23)$.

Trend of time data. Overall it was found that a random effects linear function fit the time data better than a quadratic function. The loglikelihood value associated with the random effects linear function (308705.9) was smaller than the loglikelihood value associated with the quadratic function (317417.2), and as such, the likelihood ratio test could not be ran, indicating that the linear random effects trend best fit the data. ${ }^{16}$ This indicates that the total dynamic risk scores steadily increase or decrease over time.

Unconditional means model: Variance between individuals. The intraclass correlation coefficient (ICC) was calculated and was found to be .849 (197.33/ (35.06 + 197.33)), which indicates that $84.9 \%$ of the variance in initial total dynamic risk scores can be explained by differences among justice-involved men (see Table 14).

Unconditional growth model: Level-1 change over time. As seen in Table 14, results indicated that, on average, the initial total dynamic risk score for men was 19.09 and the average rate of change was a decrease in scores by -.06 points per month while on community supervision. The variance in initial status $\left(\tau_{00}=232.78\right)$ and rate of change $\left(\tau_{11}=.27\right)$ were both significantly different from zero $(p<.001)$, indicating that there was significant variability in both initial total dynamic risk scores and in the rate of change on these scores among men. Further, the covariance between initial score and change over

\footnotetext{
${ }^{16}$ Given that the linear function fit the data better than the quadratic function, the cubic function was not examined.
} 
time $\left(\tau_{01}=-2.85, p<.001\right)$ suggests that men with higher total dynamic risk scores at initial assessment evidenced a greater decrease in scores over time relative to men with lower total dynamic risk scores at initial assessment.

Conditional model: Variation as a function of level-2 predictors. The conditional model included three level-2 predictors: age, Indigenous status, and total static risk scores. As seen in Table 14, initial total dynamic risk scores did not vary as a function of Indigenous status, however, scores did vary as a function of age $\left(\gamma_{01}=-.02\right.$, $S E=.01, p<.05)$ and total static risk score $\left(\gamma_{03}=.56, S E=.01, p<.001\right)$. Change in total dynamic risk scores, however, was found to vary as a function of age $\left(\gamma_{11}=.001, S E=\right.$ $.001, p<.05)$, Indigenous status $\left(\gamma_{12}=.05, S E=.02, p<.001\right)$, and total static risk scores $\left(\gamma_{13}=-.002, S E=.001, p<.01\right)$. However, the magnitude of these effects was extremely small. 
Table 14

Two-level Linear Growth Models Examining Changes in Total Dynamic Risk Scores Over Time for Men $(N=11,953)$

\begin{tabular}{|c|c|c|c|c|c|c|c|}
\hline & & \multicolumn{2}{|c|}{$\begin{array}{c}\text { Unconditional } \\
\text { Means }\end{array}$} & \multicolumn{2}{|c|}{$\begin{array}{c}\text { Unconditional } \\
\text { Growth }\end{array}$} & \multicolumn{2}{|c|}{$\begin{array}{c}\text { Conditional } \\
\text { Growth }\end{array}$} \\
\hline & & Estimate & $(S E)$ & Estimate & $(S E)$ & Estimate & $(S E)$ \\
\hline \multicolumn{8}{|c|}{ Fixed Effects Initial Status } \\
\hline & Intercept $\left(\gamma_{00}\right)$ & $18.72 * * *$ & $(.13)$ & $19.09 * * *$ & $(.14)$ & $19.89 * * *$ & $(.13)$ \\
\hline & Age $\left(\gamma_{01}\right)$ & -- & -- & -- & -- & $-.02 *$ & $(.01)$ \\
\hline & Indigenous $\left(\gamma_{02}\right)$ & -- & -- & -- & -- & .07 & $(.32)$ \\
\hline & Static Risk $\left(\gamma_{03}\right)$ & -- & -- & -- & -- & $.56 * * *$ & $(.01)$ \\
\hline \multicolumn{8}{|c|}{ Rate of Change } \\
\hline & Intercept $(\gamma 10)$ & -- & -- & $-.06 * * *$ & $(.01)$ & $-.12 * * *$ & $(.01)$ \\
\hline & Time*Age $(\gamma 11)$ & -- & -- & -- & -- & $.001 *$ & $(.00)$ \\
\hline & Time*Indigenous $(\gamma 12)$ & -- & -- & -- & -- & $.05 * * *$ & $(.02)$ \\
\hline & Time*Static Risk $(\gamma 13)$ & -- & -- & -- & -- & $-.002 * *$ & $(.00)$ \\
\hline \multicolumn{8}{|c|}{ Variance Components } \\
\hline Level 1 & Within Person $\left(\sigma^{2}\right)$ & $35.06 * * *$ & $(.28)$ & $17.74 * * *$ & $(.17)$ & $15.05 * * *$ & $(.15)$ \\
\hline \multirow[t]{3}{*}{ Level 2} & Initial Status $\left(\tau_{00}\right)$ & $197.33 * * *$ & $(2.68)$ & $232.78 * * *$ & $(3.17)$ & $149.65 * * *$ & $(2.09)$ \\
\hline & Rate of change $\left(\tau_{11}\right)$ & -- & -- & $.27 * * *$ & $(.01)$ & $.22 * * *$ & $(.01)$ \\
\hline & Covariance $\left(\tau_{01}\right)$ & -- & -- & $-2.85 * * *$ & $(.10)$ & $-2.60 * * *$ & $(.07)$ \\
\hline \multicolumn{8}{|l|}{ Fit Indices } \\
\hline & AIC & \multicolumn{2}{|c|}{317644.3} & \multicolumn{2}{|c|}{308707.4} & \multicolumn{2}{|c|}{296964.7} \\
\hline & $\mathrm{BIC}$ & \multicolumn{2}{|c|}{317666.5} & \multicolumn{2}{|c|}{308751.7} & \multicolumn{2}{|c|}{297053.4} \\
\hline & -2 Loglikelihood & \multicolumn{2}{|c|}{317638.3} & \multicolumn{2}{|c|}{308695.4} & \multicolumn{2}{|c|}{296940.7} \\
\hline
\end{tabular}

Note. Unconditional growth model assesses within-individual effects (level-1). Conditional growth model includes between-individual effects (level-2 predictors). Estimate $=$ regression coefficient. $S E=$ Standard Error. AIC $=$ Akaike Information Criterion. BIC $=$ Bayesian Information Criterion. Estimation Method = Maximum Likelihood; Satterthwaite degrees of freedom. ${ }^{* * *} p<.001,{ }^{* *} p<.01,{ }^{*} p<.05$ 
Although changes in total dynamic risk scores were found to vary as a function of age and total static risk scores, the magnitude of these effects was extremely small (less than .01), and as such, will not be presented in a figure. However, Figure 8 highlights the differences in changes on total dynamic risk scores over time as a function of Indigenous status. Specifically, Indigenous men had an average initial dynamic risk score of 19.94 and evidenced an average decrease in total dynamic risk scores of .10 points per month. In comparison, non-Indigenous men had an average initial dynamic risk score of 19.84 and had an average decrease in total dynamic risk scores of .13 points per month.

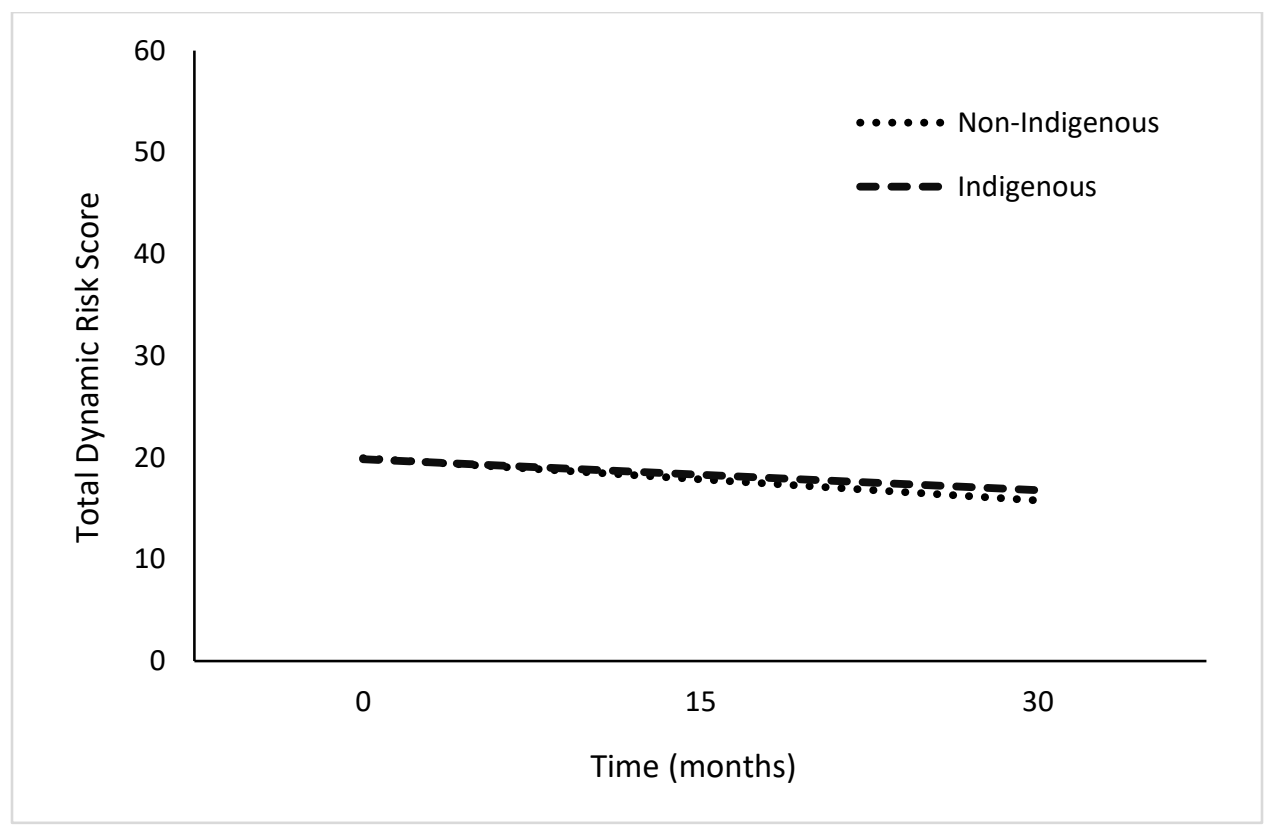

Figure 8. Men's change in total dynamic risk scores over time as a function of Indigenous status.

Figure 9 presents the initial total dynamic risk scores and the change in total dynamic risk scores over 30 months for a random sample of 1000 justice-involved men ( $8.4 \%$ of the sample). 


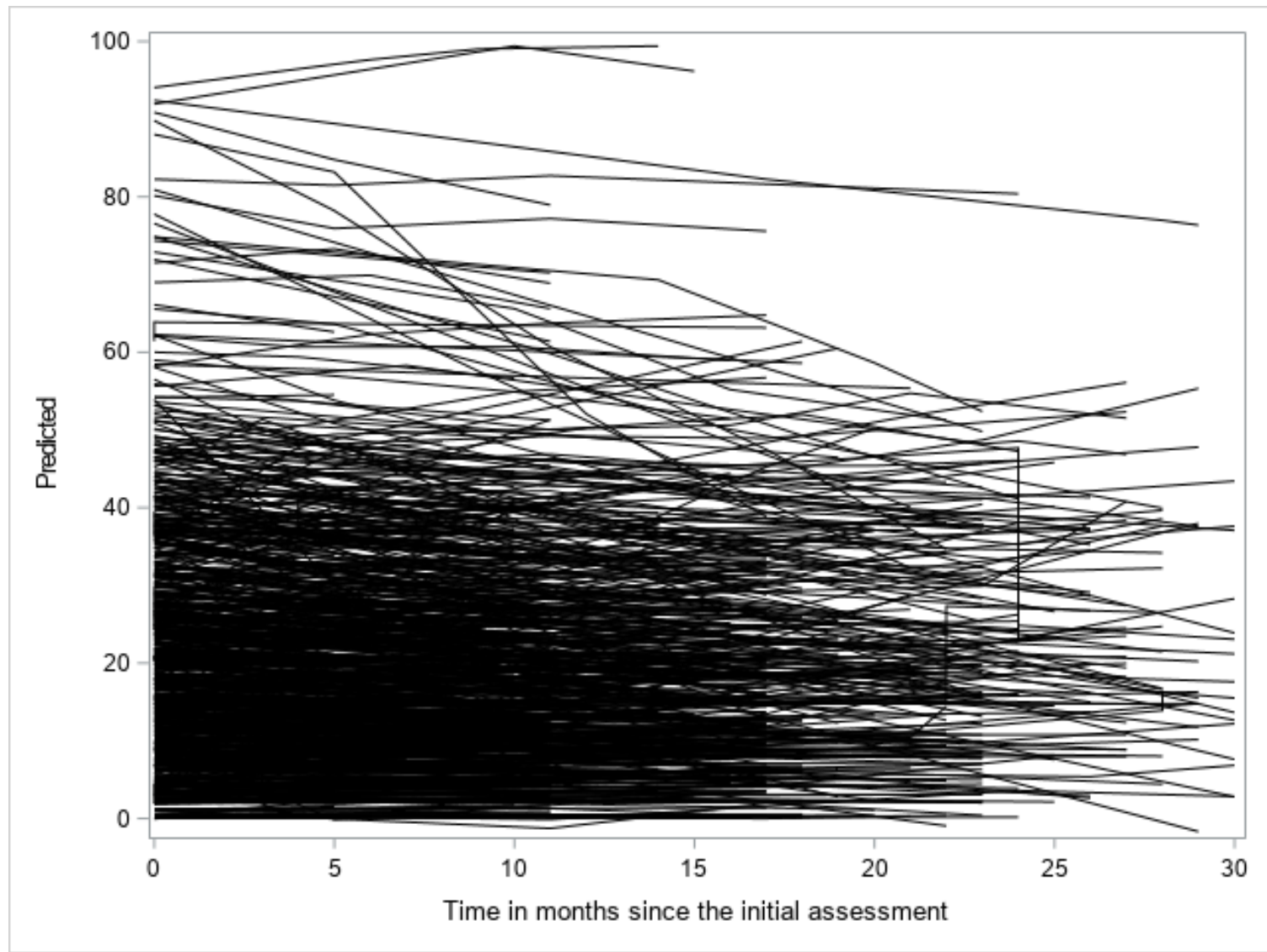

Figure 9. Individual change on total dynamic risk scores (predicted) across time in months for men.

Pseudo $\boldsymbol{R}^{2}$ results. As described in Table 15, pseudo $R^{2}$ statistics are calculated to quantify the amount of variation explained by the growth models. Results indicated that less than $1 \%$ of variance is explained by the unconditional growth model, whereas $37.9 \%$ of variance is explained by the conditional growth model. For the unconditional growth model, the proportion of within-offender variation explained by time is $49.4 \%$, whereas for the conditional growth model, the proportion of within-individual variation explained by time is $57.1 \%$. Finally, adding level- 2 predictors was found to further explain unexplained outcome variation for both the initial total dynamic risk scores $(35.7 \%)$ and the change in total dynamic risk scores over time (18.5\%). 
Table 15

Comparison of Pseudo $R^{2}$ Statistics between Unconditional and Conditional Growth Models for Men-Total Dynamic Risk Scores

\begin{tabular}{lcc}
\hline Pseudo $\boldsymbol{R}^{2}$ & $\begin{array}{c}\text { Unconditional } \\
\text { Growth }\end{array}$ & $\begin{array}{c}\text { Conditional } \\
\text { Growth }\end{array}$ \\
\hline Total Outcome & .003 & .379 \\
Within-person residual variance & .494 & .571 \\
Intercept & -- & .357 \\
Slope & -- & .185 \\
\hline Note. Unconditional growth only contains (within-person) level-1 predictors. Conditional \\
model includes (between-person) level-2 predictors.
\end{tabular}

In sum, it is evident that total dynamic risk scores overall tend to steadily decrease for men over time; however, this rate of change is quite gradual and minimal. Indigenous status did not influence the initial total dynamic risk score; however, age and static risk scores did influence initial total dynamic risk scores. Specifically, men who were younger, and men who had higher total static risk scores, had higher initial total dynamic risk scores. In contrast, age, Indigenous status and total static risk scores were found to influence rate of change on scores; however, the magnitude of the rate of change as a function of age and total static risk scores was extremely small. However, nonIndigenous men were found to have total dynamic risk scores that decreased at faster rates over time than Indigenous men. Overall, the conditional growth model explained a significant amount of variability in initial scores and change in scores over time.

Patterns of change results: Men and dynamic strength scores. The initial assessment SPIn total dynamic strength scores ranged from 0 to $89(M=27.80, S D=$ 16.40). Subsequent SPIn assessments occurred at intervals spanning 3 to 30 months post 
initial assessment and the total dynamic strength scores from these subsequent assessments ranged from 0 to $89(M=28.23, S D=16.34)$.

Trend of time data. Overall it was found that a random effects linear function fit the time data better than a quadratic function. The loglikelihood value associated with the random effects linear function (301051.3) was smaller than the loglikelihood value associated with the quadratic function (309261.5), and as such, the likelihood ratio test could not be ran, indicating that the linear random effects trend best fit the data. ${ }^{17}$ This suggests that the total dynamic strength scores steadily increase or decrease over time.

Unconditional means model: Variance between individuals. The intraclass correlation coefficient (ICC) was calculated and was found to be .903 (241.92/ (25.86 + 241.92)), which indicates that $90.3 \%$ of the variance in initial total dynamic strength scores can be explained by differences among justice-involved men (see Table 16).

Unconditional growth model: Level-1 change over time. As seen in Table 16, results indicated that, on average, the initial total dynamic strength score for men was 27.38 and the average rate of change was an increase in scores by .08 points per month while on community supervision. The variance in initial status $\left(\tau_{00}=252.56\right)$ and rate of change $\left(\tau_{11}=.21\right)$ were both significantly different from zero $(p<.001)$, indicating that there was significant variability in both initial total dynamic strength scores and in the rate of change on these scores among men. Further, the covariance between initial score and change over time $\left(\tau_{01}=-1.28, p<.001\right)$ suggests that men with lower total dynamic strength scores at initial assessment evidenced a greater increase in scores over time relative to men with higher total dynamic strength scores at initial assessment.

\footnotetext{
${ }^{17}$ Given that the linear function fit the data better than the quadratic function, the cubic function was not examined.
} 
Conditional model: Variation as a function of level-2 predictors. The

conditional model included three level-2 predictors: age, Indigenous status, and total static risk scores. As seen in Table 16, initial total dynamic strength scores varied as a function of Indigenous status, age, and total static risk scores. Notably, the largest difference in initial dynamic risk scores was a function of Indigenous status $\left(\gamma_{02}=1.72\right.$, $S E=.41, p<.001)$ and total static risk score $\left(\gamma_{03}=-.19, S E=.01, p<.001\right)$. Change in total dynamic strength scores was also found to vary as a function of age $\left(\gamma_{11}=-.001, S E\right.$ $=.001, p<.01)$, Indigenous status $\left(\gamma_{12}=-.04, S E=.02, p<.01\right)$, and total static risk scores $\left(\gamma_{13}=-.001, S E=.001, p<.01\right)$. However, the magnitude of these effects was extremely small. 
Table 16

Two-level Linear Growth Models Examining Changes in Total Dynamic Strength Scores Over Time for Men $(N=11,953)$

\begin{tabular}{|c|c|c|c|c|c|c|c|}
\hline & \multicolumn{2}{|c|}{$\begin{array}{c}\text { Unconditional } \\
\text { Means }\end{array}$} & \multicolumn{2}{|c|}{$\begin{array}{c}\text { Unconditional } \\
\text { Growth }\end{array}$} & \multicolumn{2}{|c|}{$\begin{array}{c}\text { Conditional } \\
\text { Growth }\end{array}$} \\
\hline & & Estimate & $(S E)$ & Estimate & $(S E)$ & Estimate & $(S E)$ \\
\hline \multicolumn{8}{|c|}{ Fixed Effects Initial Status } \\
\hline & Intercept $\left(\gamma_{00}\right)$ & $28.04 * * *$ & $(.14)$ & $27.38 * * *$ & $(.15)$ & $26.83 * * *$ & $(.16)$ \\
\hline & Age $\left(\gamma_{01}\right)$ & -- & -- & -- & -- & $.08 * * *$ & $(.01)$ \\
\hline & Indigenous $\left(\gamma_{02}\right)$ & -- & -- & -- & -- & $1.72 * * *$ & $(.41)$ \\
\hline & Static Risk $\left(\gamma_{03}\right)$ & -- & -- & -- & -- & $-.19 * * *$ & $(.01)$ \\
\hline \multicolumn{8}{|c|}{ Rate of Change } \\
\hline & Intercept $(\gamma 10)$ & -- & -- & $.08 * * *$ & $(.01)$ & $.10 * * *$ & $(.01)$ \\
\hline & Time*Age $(\gamma 11)$ & -- & -- & -- & -- & $-.001 * *$ & $(.00)$ \\
\hline & Time*Indigenous $(\gamma 12)$ & -- & -- & -- & -- & $-.04 * *$ & $(.02)$ \\
\hline & Time*Static Risk $(\gamma 13)$ & -- & -- & -- & -- & $-.001 * *$ & $(.00)$ \\
\hline \multicolumn{8}{|c|}{ Variance Components } \\
\hline Level 1 & Within Person $\left(\sigma^{2}\right)$ & $25.86^{* * *}$ & $(.20)$ & $12.97 * * *$ & $(.13)$ & $12.56^{* * *}$ & $(.12)$ \\
\hline \multirow[t]{3}{*}{ Level 2} & Initial Status $\left(\tau_{00}\right)$ & $241.92 * * *$ & $(3.22)$ & $252.56^{* * *}$ & (3.39) & $252.83 * * *$ & $(3.41)$ \\
\hline & Rate of change $\left(\tau_{11}\right)$ & -- & -- & $.21 * * *$ & $(.00)$ & $.20 * * *$ & $(.00)$ \\
\hline & Covariance $\left(\tau_{01}\right)$ & -- & -- & $-1.28 * * *$ & $(.09)$ & $-1.36 * * *$ & $(.09)$ \\
\hline \multicolumn{8}{|l|}{ Fit Indices } \\
\hline & AIC & \multicolumn{2}{|c|}{310116.5} & \multicolumn{2}{|c|}{301063.3} & \multicolumn{2}{|c|}{299981.0} \\
\hline & $\mathrm{BIC}$ & \multicolumn{2}{|c|}{310138.7} & \multicolumn{2}{|c|}{301107.6} & \multicolumn{2}{|c|}{300069.7} \\
\hline & -2 Loglikelihood & \multicolumn{2}{|c|}{310110.5} & \multicolumn{2}{|c|}{301051.3} & \multicolumn{2}{|c|}{299957.0} \\
\hline
\end{tabular}

Note. Unconditional growth model assesses within-individual effects (level-1). Conditional growth model includes between-individual effects (level-2 predictors). Estimate $=$ regression coefficient. $S E=$ Standard Error. AIC $=$ Akaike Information Criterion. BIC $=$ Bayesian Information Criterion. Estimation Method = Maximum Likelihood; Satterthwaite degrees of freedom. ${ }^{* * *} p<.001,{ }^{*} p<.01,{ }^{*} p<.05$ 
Although changes in total dynamic strength scores were found to vary as a function of age and total static risk scores, the magnitude of these effects was extremely small (less than .01), and as such, will not be presented in a figure. However, Figure 10 highlights the differences in changes on total dynamic strength scores over time as a function of Indigenous status. Specifically, Indigenous men had an average initial dynamic strength score of 28.03 and evidenced an average increase in total dynamic strength scores of .09 points per month. In comparison, non-Indigenous men had an average initial dynamic strength score of 25.61 and evidenced an average increase of .11 points per month.

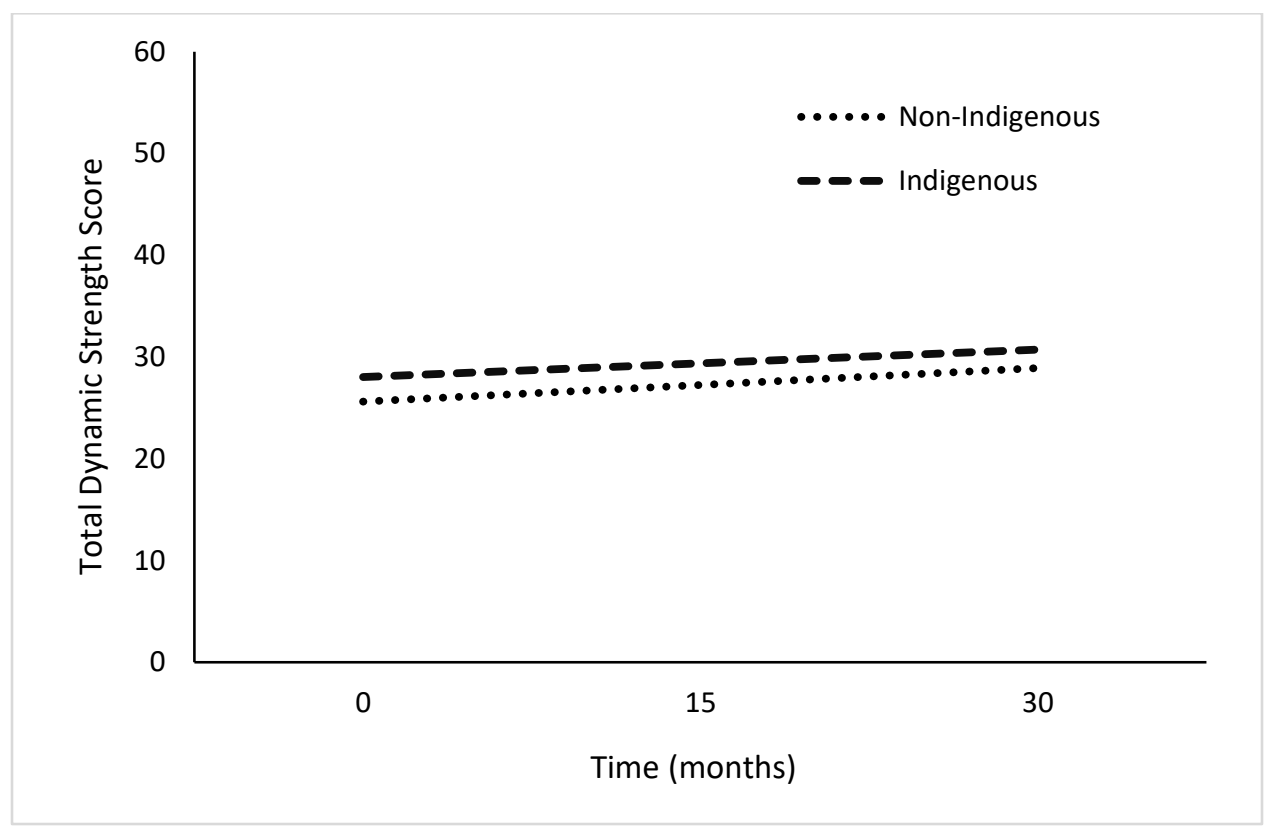

Figure 10. Men's change in dynamic strength scores over time as a function of Indigenous status.

Figure 11 presents the initial total dynamic strength scores and the change in total dynamic strength scores over 30 months for a random sample of 1,000 justice-involved men $(8.4 \%$ of the sample). 


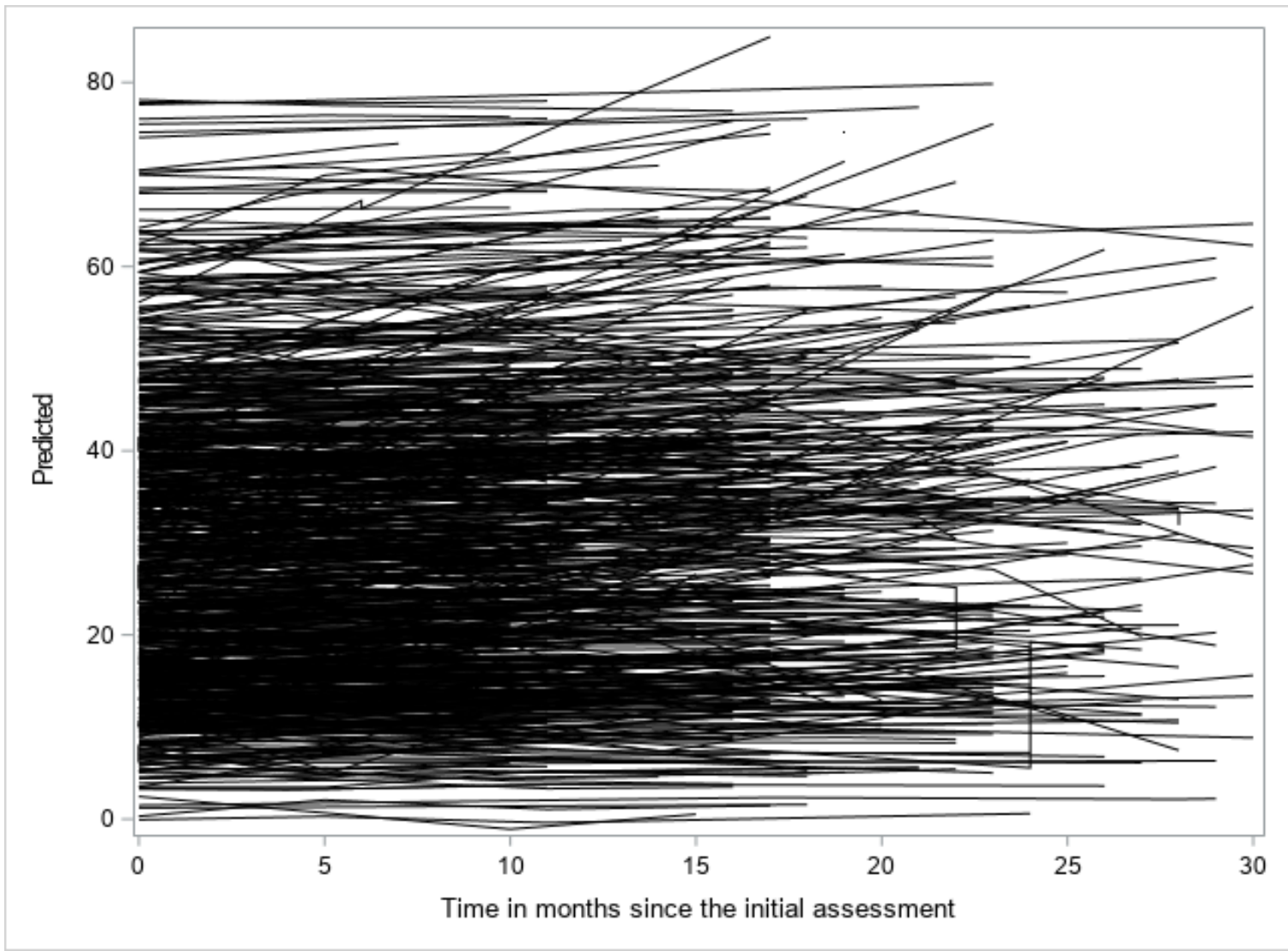

Figure 11. Individual change on total dynamic strength scores (predicted) across time in months for men.

Pseudo $\boldsymbol{R}^{2}$ results. As described in Table 17, pseudo $R^{2}$ statistics are calculated to quantify the amount of variation explained by the growth models. Results indicated that less than $1 \%$ of variance is explained by the unconditional growth model, whereas $2.1 \%$ of variance is explained by the conditional growth model. For the unconditional growth model, the proportion of within-offender variation explained by time is $49.8 \%$, whereas for the conditional growth model, the proportion of within-individual variation explained by time is $51.4 \%$. Finally, adding level-2 predictors was not found to explain unexplained outcome variation for the initial total dynamic strength scores (less than 1\%), but was 
found to explain some unexplained outcome variation on change in total dynamic strength scores over time (4.8\%).

Table 17

Comparison of Pseudo $R^{2}$ Statistics between Unconditional and Conditional Growth Models for Men-Total Dynamic Strength Scores

\begin{tabular}{ccc}
\hline Pseudo $\boldsymbol{R}^{2}$ & $\begin{array}{c}\text { Unconditional } \\
\text { Growth }\end{array}$ & $\begin{array}{c}\text { Conditional } \\
\text { Growth }\end{array}$ \\
\hline Total Outcome & .002 & .021 \\
Within-person residual variance & .498 & .514 \\
Intercept & -- & .001 \\
Slope & -- & .048 \\
\hline
\end{tabular}

Note. Unconditional growth only contains (within-person) level-1 predictors. Conditional growth model includes (between-person) level-2 predictors.

In sum, it is evident that total dynamic strength scores overall tend to steadily increase for men over time; however, this rate of change is quite gradual and minimal. Importantly, while age, Indigenous status, and total static risk scores were each found to influence the initial total dynamic strength score, the magnitude of this effect was greatest for Indigenous status whereby men who were Indigenous has significantly higher initial total dynamic strength scores than non-Indigenous men. However, Indigenous status was also found to influence change in total dynamic strength scores over time, and findings indicated that non-Indigenous men had total dynamic strength scores that increased at faster rates over time. Overall, the conditional model did not explain a significant amount of variability in both initial scores and change in scores over time. 


\section{Summary of Change Results}

Overall results of both models (dynamic risk and strength models) were similar for both men and women. For the total dynamic risk models initial scores were slightly higher for men (19.89 versus 19.20), and the rate of change was similar for both genders (-.12 for men and -.11 for women). For the strengths models, the initial assessment scores were similar, but slightly higher once again for the men than for the women (26.83 versus 26.29), and rate of change was similar for both genders (.10 for men and .11 for women). As seen in Table 18, the results of the covariates were similar for both genders with a few notable gender differences. First, for women, only the total static risk score significantly influenced the initial and change over time in total dynamic risk scores. For men, however, each of the covariates influenced initial and change scores (with the exception of Indigenous status which did not influence initial scores on total dynamic risk).

In contrast, for strengths, while each covariate significantly influenced initial total dynamic strength scores for the men, Indigenous status did not influence initial total dynamic strength score for the women. However, for both men and women, each covariate significantly influenced change in strength scores over time, with Indigenous status having the strongest influence. That is, Indigenous men had higher initial strength scores; however, the rate of change (increase in scores) was slightly faster for nonIndigenous men. For women, both Indigenous and non-Indigenous women had similar initial strength scores; however, the rate of change (increase in scores) was slightly faster for non-Indigenous women. Overall, the results indicated minimal and gradual change for both men and women and for both dynamic risk and strength scores. 
Table 18

Summary and Comparison of Results for the Dynamic Risk and Strength Models for Women and Men

\begin{tabular}{|c|c|c|c|c|}
\hline \multirow[b]{3}{*}{ Influential covariates } & \multicolumn{4}{|c|}{ Women } \\
\hline & \multicolumn{2}{|c|}{ Total Dynamic Risk } & \multicolumn{2}{|c|}{ Total Dynamic Strength } \\
\hline & $\begin{array}{c}\text { Initial score } \\
(19.20)\end{array}$ & $\begin{array}{c}\text { Change in scores } \\
(-.11)\end{array}$ & $\begin{array}{c}\text { Initial score } \\
(26.29)\end{array}$ & $\begin{array}{c}\text { Change in scores } \\
(.11)\end{array}$ \\
\hline Age & $x$ & $x$ & $\checkmark$ & $\checkmark$ \\
\hline Indigenous Status & $x$ & $x$ & $x$ & $\checkmark$ \\
\hline \multirow[t]{3}{*}{ Static Risk } & $\checkmark$ & $\checkmark$ & $\checkmark$ & $\checkmark$ \\
\hline & \multicolumn{4}{|c|}{ Men } \\
\hline & \multicolumn{2}{|c|}{ Total Dynamic Risk } & \multicolumn{2}{|c|}{ Total Dynamic Strength } \\
\hline Influential covariates & $\begin{array}{c}\text { Initial score } \\
(19.89)\end{array}$ & $\begin{array}{c}\text { Change in scores } \\
(-.12)\end{array}$ & $\begin{array}{c}\text { Initial score } \\
(26.83)\end{array}$ & $\begin{array}{c}\text { Change in scores } \\
(.10)\end{array}$ \\
\hline Age & $\checkmark$ & $\checkmark$ & $\checkmark$ & $\checkmark$ \\
\hline Indigenous Status & $x$ & $\checkmark$ & $\checkmark$ & $\checkmark$ \\
\hline Static Risk & $\checkmark$ & $\checkmark$ & $\checkmark$ & $\checkmark$ \\
\hline
\end{tabular}

Note. $\checkmark=$ covariate influence on dynamic domain. $\mathrm{X}=$ covariate does not influence dynamic domain. 


\section{Using Offender Change in Dynamic Strengths and Risks to Predict Outcome}

To examine how the previously assessed patterns of change in dynamic needs and dynamic strengths relate to criminal outcome, a two-stage HLM was used, which is an extension from basic HLM. ${ }^{18}$ The two-stage HLM model (TSM) is based off of three advanced statistical models which are commonly used in epidemiological, life-course analysis; generalized linear models, HLM, and SEM (Yang et al., 2017). Although this approach has not been widely used in forensic and criminal justice psychology, Yang and colleagues (2017) argue that this approach can account for various challenges faced in the field of correctional risk assessment. Some of these challenges include irregular data collection intervals (risk assessment periods), missing data, drop-outs during follow-up, and different data collectors (e.g., different correctional personnel). Specifically, TSM is useful when repeated measures occur at different time intervals and have missing measurements (Yang et al., 2017).

TSM is conducted in two stages. Stage one is hierarchical linear growth modeling, as conducted in the previous section. Stage two involves running a regression model using the change parameters (the intercepts and slopes) calculated during the HLM stage to link these change patterns to distal outcomes (Yang et al., 2017). Distal outcomes in this context refer to the reoffending outcomes (any new charge, technical violation, violent charge, and any negative outcome_-new charge or technical violation).

\footnotetext{
${ }^{18}$ Other statistical approaches can be used to examine recidivism prediction over time. For instance, Cox regression applies regression techniques to survival data (length of time until an event occurs) (Tabachnick \& Fidell, 2007), allowing for multiple predictors to be assessed at the same time to determine their unique contributions to outcome (e.g., recidivism). Cox regression also estimates relative risk ratios which indicates the likelihood of an event (e.g., recidivism; Tabachnick \& Fidell, 2007). While Cox regression is unique as it allows for researchers to examine whether assessments that are more recent predict recidivism over and above the initial assessment (Lloyd, 2015), it does not allow for the examination of how individuals change or how patterns of change differ for individuals.
} 
Specifically, the intercept and slopes are input into a regression equation-in this case, logistic regression given that the four outcomes of interest are each dichotomous. Parameter estimates from the conditional model that included between-individual effects (level-2 predictors such as Indigenous status, age, and static risk scores) were retained. As such, these covariates were not entered again into the second stage of the TSM to avoid accounting for them twice in the change model parameter estimates. Total dynamic risk and dynamic strength models were ran independently for men and women to explain potential gender differences.

Criminal outcome for women and men. There were four outcomes of interest: any negative outcome (includes technical violations and criminal justice system recontact), any violent charges (including crimes against the person such as harassment, threats, assaults, sexual offences and weapons offences), any new charges (which includes violent charges and non-violent charges such as property and drug-related offences), and technical violations. Outcomes by gender are presented in Table 19. Overall, approximately $32 \%$ of women had a negative outcome of some sort. Interestingly, more women had a new charge over a three-year fixed follow-up than a technical violation ( $25 \%$ versus $20 \%$ ). In comparison, for the full sample of men, approximately $36 \%$ had negative outcome of some sort. Similar to the women, more men had a new charge than a technical violation (29\% versus $23 \%)$. Overall, men had significantly more new charges and technical violations than women with the biggest difference found for violent charges, and the smallest difference found for technical violations. 
Table 19

Frequency of Men and Women who Reoffended Over a Three-Year Fixed Follow-Up

\begin{tabular}{lccccccc}
\hline \multirow{2}{*}{ Outcome } & \multicolumn{2}{c}{$\begin{array}{c}\text { Women } \\
(\boldsymbol{N}=\mathbf{2 , 8 7 7})\end{array}$} & \multicolumn{2}{c}{$\begin{array}{c}\text { Men } \\
(\boldsymbol{N}=\mathbf{1 1 , 9 5 3})\end{array}$} & & \\
\cline { 2 - 5 } & $\boldsymbol{n}$ & $\mathbf{\%}$ & $\boldsymbol{n}$ & $\mathbf{\%}$ & $\chi^{2}$ & \multirow{d}{*}{} \\
\hline Any negative outcome & 932 & 32.4 & 4,326 & 36.2 & $14.61^{*}$ & .06 \\
Any new change & 716 & 24.9 & 3,478 & 29.1 & $20.27^{*}$ & .07 \\
New violent charge & 292 & 10.1 & 1,940 & 16.2 & $67.06^{*}$ & .14 \\
Technical violation & 568 & 19.7 & 2,729 & 22.8 & $12.79^{*}$ & .06
\end{tabular}

Note. $\chi^{2}=$ Chi-square; $d=$ Cohen's $d$ effect size. Any negative outcome refers to any new offence or any technical violation. TV $=$ Technical violation. ${ }^{*} p<.001$

Using change to predict criminal outcomes for women. The average rate of change (slopes) and initial scores (intercept) for women are presented in Table $20 .{ }^{19}$ This descriptive information provides insight around the average initial scores and rate of change scores, as well as the amount of variation around these scores.

Table 20

Average Rate of Change and Initial Total Dynamic Risk and Strength Scores for Women

\begin{tabular}{lcc}
\hline SPIn Scores & $\boldsymbol{M}$ & $(\boldsymbol{S D})$ \\
\hline Rate of Change & & \\
$\quad$ Dynamic risk & -.107 & $(.356)$ \\
$\quad$ Dynamic strength & .108 & $(.342)$ \\
Initial Scores & & \\
$\quad$ Dynamic risk & 19.20 & $(11.92)$ \\
$\quad$ Dynamic strength & 26.29 & $(15.93)$ \\
\hline
\end{tabular}

Note. $M=$ Mean; $S D=$ Standard Deviation

\footnotetext{
${ }^{19}$ The average slope presented in Table 20 are based on equally weighted individual offenders' slopes, whereas in HLGM analyses more weight is given to individuals' slopes that are based on more timepoints and whose first timepoint is closer to time zero (Babchishin, 2013).
} 
Logistic regression results - Total dynamic risk scores. The results of each of the four logistic regression analyses are presented narratively in turn and in Table 21.

Predicting any negative outcome. The initial total dynamic risk score was a significant predictor of any negative outcome, $b=.017, S E=.004$, Wald's $\chi^{2}=21.25, p<$ $.001, \mathrm{OR}=1.017(95 \% \mathrm{CI}[1.010,1.025])$, whereby individuals with higher initial total dynamic risk scores were more likely to have a negative outcome of some sort. The change over time was found to be a stronger predictor than the initial score, $b=.375, S E$ $=.125$, Wald's $\chi^{2}=9.07, p=.003, \mathrm{OR}=1.455(95 \% \mathrm{CI}[1.140,1.857])$, where those whose total dynamic risk scores increased over time were more likely to have a negative outcome than those whose scores decreased over time.

Predicting any new charge. The initial total dynamic risk score was a significant predictor of new charges, $b=.012, S E=.004$, Wald's $\chi^{2}=8.79, p=.003, \mathrm{OR}=1.012$ (95\% CI [1.004, 1.020]), whereby higher initial total dynamic risk scores were more likely to be charged for a new offence. Change in total dynamic risk scores over time was also a significant predictor of new charges, $b=.289, S E=.133$, Wald's $\chi^{2}=4.70, p=$ $.030, \mathrm{OR}=1.335(95 \% \mathrm{CI}[1.028,1.734])$. This indicates that those whose scores increased over time were more likely to be charged for a new offence than those whose total dynamic risk scores decreased over time.

Predicting any new violent charge. The initial total dynamic risk score was a significant predictor of new violent charges, $b=.013, S E=.006$, Wald's $\chi^{2}=5.93, p<$ $.001, \mathrm{OR}=1.014(95 \% \mathrm{CI}[1.003,1.025])$, whereby higher initial total dynamic risk scores were more likely to be charged for a violent offence. Change in total dynamic risk 
scores over time was not a significant predictor of new violent charges, $b=.252, S E=$ .185, Wald's $\chi^{2}=1.86, p=.173, \mathrm{OR}=1.287(95 \%$ CI $[.895,1.850])$.

Predicting a technical violation. The initial total dynamic risk score was a significant predictor of technical violations, $b=.027, S E=.004$, Wald's $\chi^{2}=39.48, p<$ $.001, \mathrm{OR}=1.027(95 \% \mathrm{CI}[1.018,1.035])$. Although the initial total dynamic risk score was predictive of technical violations, change in scores over time was a stronger predictor, $b=.677, S E=.143$, Wald's $\chi^{2}=22.56, p<.001, \mathrm{OR}=1.967$ (95\% CI [1.488, 2.601]), whereby those whose total dynamic risk scores increased over time were more likely to commit a technical violation than those whose scores decreased over time. 
Table 21

Logistic Regression Results: Predicting Criminal Outcome using Change in Total Dynamic Risk Scores for Women

\begin{tabular}{|c|c|c|c|c|c|c|c|}
\hline \multicolumn{8}{|c|}{ Any negative outcome } \\
\hline Effect & & $\boldsymbol{b}$ & $S E$ & $\chi^{2}$ & $p$ & Odds Ratio & $95 \% \mathrm{CI}$ \\
\hline & Initial Score & .017 & .004 & 21.25 & $<.001$ & 1.017 & {$[1.010,1.025]$} \\
\hline & Change over time & .375 & .125 & 9.07 & .003 & 1.455 & {$[1.140,1.857]$} \\
\hline \multicolumn{8}{|c|}{ Any new charge } \\
\hline Effect & & $b$ & $S E$ & $\chi^{2}$ & $p$ & Odds Ratio & $95 \% \mathrm{CI}$ \\
\hline & Initial Score & .012 & .004 & 8.79 & .003 & 1.012 & {$[1.004,1.020]$} \\
\hline & Change over time & .289 & .133 & 4.70 & .030 & 1.335 & {$[1.028,1.734]$} \\
\hline \multicolumn{8}{|c|}{ New violent charge } \\
\hline Effect & & $b$ & $S E$ & $\chi^{2}$ & $p$ & Odds Ratio & $95 \% \mathrm{CI}$ \\
\hline & Initial Score & .013 & .006 & 5.93 & .015 & 1.014 & {$[1.003,1.025]$} \\
\hline & Change over time & .252 & .185 & 1.86 & .173 & 1.287 & {$[0.895,1.850]$} \\
\hline \multicolumn{8}{|c|}{ Technical violation } \\
\hline Effect & & 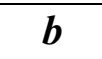 & $S E$ & $\chi^{2}$ & $p$ & Odds Ratio & $95 \% \mathrm{CI}$ \\
\hline & Initial Score & .027 & .004 & 39.48 & $<.001$ & 1.027 & {$[1.018,1.035]$} \\
\hline & Change over time & .677 & .143 & 22.56 & $<.001$ & 1.967 & {$[1.488,2.601]$} \\
\hline
\end{tabular}

Note. $b=$ beta coefficient; $S E=$ Standard error; $\chi^{2}=$ Chi-square; $\mathrm{CI}=$ Confidence Interval. Any negative outcome refers to any new offence or any new technical violation. 
Logistic regression results - Total dynamic strength scores. The results of the logistic regressions for each outcome are presented narratively and in Table 22.

Predicting negative outcome. Initial total dynamic strength score significantly predicted negative outcomes, $b=-.007, S E=.003$, Wald's $\chi^{2}=6.55, p=.011, \mathrm{OR}=.993$ $(95 \%$ CI $[.988, .998])$, whereby lower initial scores were more likely to have a negative outcome. Change in total dynamic strength scores did not predict negative outcomes, $b=$ $.017, S E=.119$, Wald's $\chi^{2}=.022, p=.883, \mathrm{OR}=1.018(95 \%$ CI $[.806,1.284])$.

Predicting any new charge. Both the initial total dynamic strength score $(b=$ $.005, S E=.003$, Wald's $\chi^{2}=3.68, p=.055, \mathrm{OR}=0.995,95 \%$ CI $\left.[0.989,1.000]\right)$, and the change in total dynamic strength scores over time $\left(b=.080, S E=.128\right.$, Wald's $\chi^{2}=0.39$, $p=.531, \mathrm{OR}=1.083,95 \% \mathrm{CI}[0.844,1.391])$, did not predict new charges

Predicting any new violent charge. The initial total dynamic strength score was a significant predictor of new violent charges, $b=-.009, S E=.004$, Wald's $\chi^{2}=4.73, p=$ $.030, \mathrm{OR}=0.991(95 \% \mathrm{CI}[0.983,0.999])$, whereby those with higher scores were less likely to be charged for a violent offence. Change in total dynamic strength scores over time did not predict violent charges, $b=-.289, S E=.193$, Wald's $\chi^{2}=2.24, p=.135$, OR $=0.749(95 \%$ CI $[0.513,1.094])$.

Predicting a technical violation. The initial total dynamic strength score was a significant predictor of technical violations, $b=-.008, S E=.003$, Wald's $\chi^{2}=6.09, p=$ $.014, \mathrm{OR}=0.992(95 \% \mathrm{CI}[0.986,0.998])$. Change in total dynamic strength scores over time was a stronger predictor, $b=-.609, S E=.152$, Wald's $\chi^{2}=16.18, p<.001, \mathrm{OR}=$ $0.544(95 \%$ CI $[0.404,0.732])$, whereby those whose scores increased over time were less likely to have a technical violation. 
Table 22

Logistic Regression Results: Predicting Criminal Outcome using Change in Total Dynamic Strength Scores for Women

\begin{tabular}{|c|c|c|c|c|c|c|c|}
\hline \multicolumn{8}{|c|}{ Any negative outcome } \\
\hline Effect & & $b$ & $S E$ & $\chi^{2}$ & $p$ & Odds Ratio & $95 \% \mathrm{CI}$ \\
\hline & Initial Score & -.007 & .003 & 6.55 & .011 & 0.993 & {$[0.988,0.998]$} \\
\hline & Change over time & .017 & .119 & 0.02 & .883 & 1.018 & {$[0.806,1.284]$} \\
\hline \multicolumn{8}{|c|}{ Any new charge } \\
\hline Effect & & $b$ & $S E$ & $\chi^{2}$ & $p$ & Odds Ratio & $95 \% \mathrm{CI}$ \\
\hline & Initial Score & -.005 & .003 & 3.68 & .055 & 0.995 & {$[0.989,1.000]$} \\
\hline & Change over time & .080 & .128 & 0.39 & .531 & 1.083 & {$[0.844,1.391]$} \\
\hline \multicolumn{8}{|c|}{ New violent charge } \\
\hline Effect & & $b$ & $S E$ & $\chi^{2}$ & $p$ & Odds Ratio & $95 \% \mathrm{CI}$ \\
\hline & Initial Score & -.009 & .004 & 4.73 & .030 & 0.991 & {$[0.983,0.999]$} \\
\hline & Change over time & -.289 & .193 & 2.24 & .135 & 0.749 & {$[0.513,1.094]$} \\
\hline \multicolumn{8}{|c|}{ Technical violation } \\
\hline Effect & & 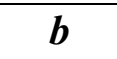 & $S E$ & $\chi^{2}$ & $p$ & Odds Ratio & $95 \% \mathrm{CI}$ \\
\hline & Initial Score & -.008 & .003 & 6.09 & .014 & 0.992 & {$[0.986,0.998]$} \\
\hline & Change over time & -.609 & .152 & 16.18 & $<.001$ & 0.544 & {$[0.404,0.732]$} \\
\hline
\end{tabular}

Note. $b=$ beta coefficient; $S E=$ Standard error; $\chi^{2}=$ Chi-square; $\mathrm{CI}=$ Confidence Interval. Any negative outcome refers to any new offence or any new technical violation. 
Using change to predict criminal outcomes for men. It is important to note that because of the large number of observations/large sample size of men, the parameter estimates could not be saved for the entire sample due to program constraints (i.e., insufficient memory of the statistical program). As such, a random sample of men was selected. Specifically, a subsample of 3,845 men (13,800 observations) was included, which was the maximum number of observations that could be analyzed by the program. In order to ensure that the random subsample was representative of the entire sample of men, potential differences were examined for the various predictor, covariate, and outcome variables. As seen in Table 23, there were no significant differences across all of the variables of importance, indicating that the subsample was representative of the full sample and any differences between the subsample and full sample are minimal. 
Table 23

Comparing the Subsample to the Full Sample of Justice-Involved Men used for Analyses

\begin{tabular}{|c|c|c|c|c|c|c|}
\hline \multirow[t]{2}{*}{ Continuous Variables } & \multicolumn{2}{|c|}{$\begin{array}{l}\text { Full sample } \\
(N=11,953)\end{array}$} & \multicolumn{2}{|c|}{$\begin{array}{l}\text { Subsample } \\
(n=3,845)\end{array}$} & \multirow[b]{2}{*}{$t$} & \multirow[b]{2}{*}{$p$} \\
\hline & $M$ & $S D$ & $M$ & $S D$ & & \\
\hline Static Risk $^{1}$ & 25.0 & $(18.8)$ & 24.8 & $(18.8)$ & 0.60 & .547 \\
\hline Dynamic Risk ${ }^{2}$ & 19.3 & $(15.8)$ & 19.3 & $(15.9)$ & 0.10 & .919 \\
\hline Dynamic Strength ${ }^{3}$ & 27.8 & $(16.4)$ & 28.3 & $(16.6)$ & -1.63 & .103 \\
\hline Age & 33.6 & $(11.4)$ & 33.7 & $(11.6)$ & -0.47 & .641 \\
\hline \multirow[t]{2}{*}{ Categorical Variables } & \multicolumn{2}{|c|}{$\begin{array}{l}\text { Full sample } \\
(N=11,953)\end{array}$} & \multicolumn{2}{|c|}{$\begin{array}{l}\text { Subsample } \\
(n=3,845)\end{array}$} & & \\
\hline & $n$ & $\%$ & $n$ & $\%$ & $\chi^{2}$ & $p$ \\
\hline Indigenous & 1,988 & $(16.6)$ & 648 & (16.9) & 0.10 & .749 \\
\hline Any Negative & 4,326 & $(36.2)$ & 1,426 & $(37.1)$ & 1.01 & .316 \\
\hline Technical Violation & 2,729 & $(22.8)$ & 902 & $(23.5)$ & 0.65 & .421 \\
\hline Any New Charge & 3,478 & $(29.1)$ & 1,144 & $(29.8)$ & 0.60 & .437 \\
\hline Violent Charge & 1,940 & $(16.2)$ & 639 & $(16.6)$ & 0.32 & .570 \\
\hline
\end{tabular}

Note. $M=$ mean; $S D=$ standard deviation; $t=\mathrm{t}$-test; $\chi^{2}=$ Chi-square. ${ }^{1}$ Static risk refers to the SPIn Full Assessment total static risk score. ${ }^{2}$ Dynamic risk refers to the SPIn Full Assessment total dynamic risk score. ${ }^{3}$ Dynamic strength refers to the SPIn Full Assessment total dynamic strength score.

The average rate of change and initial scores for both total dynamic risk and total dynamic strength scores for men are presented in Table 24.

Table 24

Average Rate of Change and Initial Dynamic Risk and Strength Scores for Men

\begin{tabular}{lcc}
\hline SPIn Total Scores & $\boldsymbol{M}$ & (SD) \\
\hline Rate of Change & & \\
Dynamic risk & -.126 & $(.402)$ \\
$\quad$ Dynamic strength & .101 & $(.385)$ \\
Initial Scores & & \\
Dynamic risk & 19.79 & $(12.13)$ \\
$\quad$ Dynamic strength & 27.24 & $(15.97)$ \\
\hline
\end{tabular}

Note. $M=$ Mean; $S D=$ Standard Deviation 
Logistic regression results - Total dynamic risk scores. The results of the logistic regressions for each of the four criminal outcomes are presented below. Results are further illustrated in Table 25.

Predicting any negative outcome. The initial total dynamic risk score was a significant predictor of negative outcomes, $b=.017, S E=.003$, Wald's $\chi^{2}=25.93, p<$ $.001, \mathrm{OR}=1.017(95 \% \mathrm{CI}[1.010,1.023])$, whereby higher initial scores were more likely to have a negative outcome. The change over time was found to be a stronger predictor than the initial score, $b=.542, S E=.101$, Wald's $\chi^{2}=28.89, p<.001, \mathrm{OR}=1.720(95 \%$ CI $[1.411,2.096])$, where those whose total dynamic risk scores increased over time were more likely to have any negative outcome than those whose scores decreased over time.

Predicting any new charge. The initial total dynamic risk score was a significant predictor of new charges, $b=.011, S E=.003$, Wald's $\chi^{2}=11.32, p=.001, \mathrm{OR}=1.011$ (95\% CI $[1.005,1.018])$, whereby those with higher initial scores were more likely to be charged with a new offence. Change in total dynamic risk scores over time was a stronger predictor of any new charges, $b=.442, S E=.105$, Wald's $\chi^{2}=17.72, p<.001, \mathrm{OR}=$ $1.556(95 \%$ CI $[1.267,1.912])$. This indicates that those whose scores increased over time were more likely to be charged with a new offence.

Predicting any new violent charge. The initial total dynamic risk score was a significant predictor of violent charges, $b=.014, S E=.004$, Wald's $\chi^{2}=12.23, p=.001$, $\mathrm{OR}=1.014(95 \% \mathrm{CI}[1.006,1.022])$, whereby higher initial scores were more likely to be charged for a new violent offence. Change in total dynamic risk scores over time was a stronger predictor, $b=.355, S E=.124$, Wald's $\chi^{2}=8.13, p=.004, \mathrm{OR}=1.426(95 \% \mathrm{CI}$ 
$[1.117,1.819])$. This indicates that those whose scores increased over time were more likely to be charged with a new violent offence.

Predicting a technical violation. Finally, similar to the other outcomes, both the initial total dynamic risk score $\left(b=.027, S E=.004\right.$, Wald's $\chi^{2}=56.99, p<.001, \mathrm{OR}=$ $1.028,95 \%$ CI $[1.020,1.035])$, and change in scores over time $(b=.732, S E=.113$, Wald's $\chi^{2}=42.22, p<.001, \mathrm{OR}=2.078,95 \%$ CI $\left.[1.667,2.592]\right)$, were predictive of technical violations. Specifically, those whose total dynamic risk scores were higher or increased over time were more likely to be charged with a technical violation. 
Table 25

Logistic Regression Results: Predicting Criminal Outcome using Change in Total Dynamic Risk Scores for Men

\begin{tabular}{|c|c|c|c|c|c|c|c|}
\hline \multicolumn{8}{|c|}{ Any negative outcome } \\
\hline Effect & & $\boldsymbol{b}$ & $S E$ & $\chi^{2}$ & $p$ & Odds Ratio & $95 \% \mathrm{CI}$ \\
\hline & Initial Score & .017 & .003 & 25.93 & $<.001$ & 1.017 & {$[1.010,1.023]$} \\
\hline & Change over time & .542 & .101 & 28.89 & $<.001$ & 1.720 & {$[1.411,2.096]$} \\
\hline \multicolumn{8}{|c|}{ Any new charge } \\
\hline Effect & & $b$ & $S E$ & $\chi^{2}$ & $p$ & Odds Ratio & $95 \% \mathrm{CI}$ \\
\hline & Initial Score & .011 & .003 & 11.32 & .001 & 1.011 & {$[1.005,1.018]$} \\
\hline & Change over time & .442 & .105 & 17.72 & $<.001$ & 1.556 & {$[1.267,1.912]$} \\
\hline \multicolumn{8}{|c|}{ New violent charge } \\
\hline Effect & & $b$ & $S E$ & $\chi^{2}$ & $p$ & Odds Ratio & $95 \% \mathrm{CI}$ \\
\hline & Initial Score & .014 & .004 & 12.23 & .001 & 1.014 & {$[1.006,1.022]$} \\
\hline & Change over time & .355 & .124 & 8.13 & .004 & 1.426 & {$[1.117,1.819]$} \\
\hline \multicolumn{8}{|c|}{ Technical violation } \\
\hline Effect & & 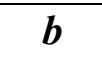 & $S E$ & $\chi^{2}$ & $p$ & Odds Ratio & $95 \% \mathrm{CI}$ \\
\hline & Initial Score & .027 & .004 & 56.99 & $<.001$ & 1.028 & {$[1.020,1.035]$} \\
\hline & Change over time & .732 & .113 & 42.22 & $<.001$ & 2.078 & {$[1.667,2.592]$} \\
\hline
\end{tabular}

Note. $b=$ beta coefficient; $S E=$ Standard error; $\chi^{2}=$ Chi-square; $\mathrm{CI}=$ Confidence Interval. Any negative outcome refers to any new offence or any new technical violation. 
Logistic regression results - Total dynamic strength scores. The results of the logistic regressions for each criminal outcome are presented below (see Table 26).

Predicting any negative outcome. Both the initial total dynamic strength score $(b$ $=-.004, S E=.002$, Wald's $\chi^{2}=2.96, p=.086, \mathrm{OR}=0.996,95 \%$ CI $\left.[.992,1.001]\right)$, and the change in scores over time $\left(b=-.167, S E=.089\right.$, Wald's $\chi^{2}=3.52, p=.061, \mathrm{OR}=$ $0.846,95 \%$ CI $[0.711,1.007])$, were not found to predict any negative outcomes.

Predicting any new charge. The initial total dynamic strength score $(b=-.006$, $S E=.003$, Wald's $\chi^{2}=4.91, p=.027, \mathrm{OR}=0.994,95 \%$ CI [0.988, 0.999]), was predictive of new charges, whereby those with higher scores were less likely to be charged with a new offence. The change in scores over time, however, did not predict new charges $\left(b=.151, S E=.112\right.$, Wald's $\chi^{2}=1.82, p=.178, \mathrm{OR}=1.163,95 \% \mathrm{CI}$ $[0.934,1.448])$.

Predicting any new violent charge. Both the initial total dynamic strength score $\left(b=-.002, S E=.002\right.$, Wald's $\chi^{2}=1.19, p=.276, \mathrm{OR}=0.998,95 \%$ CI $\left.[0.993,1.002]\right)$, and the change in scores over time did not predict new violent charges $(b=-.083, S E=$ .093 , Wald's $\left.\chi^{2}=.791, p=.374, \mathrm{OR}=0.920,95 \% \mathrm{CI}[0.766,1.105]\right)$.

Predicting a technical violation. The initial total dynamic strength score was a significant predictor of technical violations, $b=-.006, S E=.002$, Wald's $\chi^{2}=6.50, p=$ $.011, \mathrm{OR}=0.994(95 \% \mathrm{CI}[0.989,0.999])$. Notably, change in scores over time was a stronger predictor, $b=-.407, S E=.106$, Wald's $\chi^{2}=14.62, p<.001, \mathrm{OR}=0.666(95 \%$ CI $[0.541,0.820])$, whereby those whose total dynamic strength scores increased over time were less likely to be charged with a technical violation. 
Table 26

Logistic Regression Results: Predicting Criminal Outcome using Change in Total Dynamic Strength Scores for Men

\begin{tabular}{|c|c|c|c|c|c|c|c|}
\hline \multicolumn{8}{|c|}{ Any negative outcome } \\
\hline Effect & & $\boldsymbol{b}$ & $S E$ & $\chi^{2}$ & $p$ & Odds Ratio & $95 \% \mathrm{CI}$ \\
\hline & Initial Score & -.004 & .002 & 2.96 & .086 & 0.996 & {$[0.992,1.001]$} \\
\hline & Change over time & -.167 & .089 & 3.52 & .061 & 0.846 & {$[0.711,1.007]$} \\
\hline \multicolumn{8}{|c|}{ Any new charge } \\
\hline Effect & & $b$ & $S E$ & $\chi^{2}$ & $p$ & Odds Ratio & $95 \% \mathrm{CI}$ \\
\hline & Initial Score & -.006 & .003 & 4.91 & .027 & 0.994 & {$[0.988,0.999]$} \\
\hline & Change over time & .151 & .112 & 1.82 & .178 & 1.163 & {$[0.934,1.448]$} \\
\hline \multicolumn{8}{|c|}{ New violent charge } \\
\hline Effect & & $b$ & $S E$ & $\chi^{2}$ & $p$ & Odds Ratio & $95 \% \mathrm{CI}$ \\
\hline & Initial Score & -.002 & .002 & 1.19 & .276 & 0.998 & {$[0.993,1.002]$} \\
\hline & Change over time & -.083 & .093 & 0.79 & .374 & 0.920 & {$[0.766,1.105]$} \\
\hline \multicolumn{8}{|c|}{ Technical violation } \\
\hline Effect & & 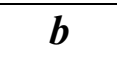 & $S E$ & $\chi^{2}$ & $p$ & Odds Ratio & $95 \% \mathrm{CI}$ \\
\hline & Initial Score & -.006 & .002 & 6.50 & .011 & 0.994 & {$[0.989,0.999]$} \\
\hline & Change over time & -.407 & .106 & 14.62 & $<.001$ & 0.666 & {$[0.541,0.820]$} \\
\hline
\end{tabular}

Note. $b=$ beta coefficient; $S E=$ Standard error; $\chi^{2}=$ Chi-square; $\mathrm{CI}=$ Confidence Interval. Any negative outcome refers to any new offence or any new technical violation. 


\section{Summary of Prediction Results}

Overall prediction results for total dynamic risk and strength models were quite similar for both men and women (see Table 27 for summary of findings). For the total dynamic risk models initial scores were predictive of all four outcomes — any new charges, any violent charges, any negative outcome, and technical violations, for both men and women. For rate of change in total dynamic risk scores, the change score was predictive of all four outcomes for men. However, for women it was not predictive of any new violent charges, but was predictive of the three other outcomes. For the strengths models, the initial assessment scores were predictive of any new charges and technical violations for the men, but not violent charges or any negative outcome. However, for the women, initial strength scores were predictive of all outcomes with the exception of any new charges. Although there were some differences in prediction of initial strengths scores for men and women, the prediction results of the change in strengths scores were similar for men and women. Specifically, change in total dynamic strength scores were only predictive of technical violations. 
Table 27

Summary and Comparison of Prediction Results of Total Dynamic Risk and Strength Scores for Men and Women

\begin{tabular}{|c|c|c|c|c|}
\hline \multirow[b]{3}{*}{ Reoffending outcomes } & \multicolumn{4}{|c|}{ Women } \\
\hline & \multicolumn{2}{|c|}{ Total Dynamic Risk } & \multicolumn{2}{|c|}{ Total Dynamic Strength } \\
\hline & Initial score & Change in scores & Initial score & Change in scores \\
\hline Any negative outcome & $\checkmark$ & $\checkmark$ & $\checkmark$ & $x$ \\
\hline Any new charge & $\checkmark$ & $\checkmark$ & $x$ & $x$ \\
\hline Any violent charge & $\checkmark$ & $x$ & $\checkmark$ & $x$ \\
\hline \multirow[t]{3}{*}{ Technical violation } & $\checkmark$ & $\checkmark$ & $\checkmark$ & $\checkmark$ \\
\hline & \multicolumn{4}{|c|}{ Men } \\
\hline & \multicolumn{2}{|c|}{ Total Dynamic Risk } & \multicolumn{2}{|c|}{ Total Dynamic Strength } \\
\hline Reoffending outcomes & Initial score & Change in scores & Initial score & Change in scores \\
\hline Any negative outcome & $\checkmark$ & $\checkmark$ & $x$ & $x$ \\
\hline Any new charge & $\checkmark$ & $\checkmark$ & $\checkmark$ & $x$ \\
\hline Any violent charge & $\checkmark$ & $\checkmark$ & $x$ & $x$ \\
\hline Technical violation & $\checkmark$ & $\checkmark$ & $\checkmark$ & $\checkmark$ \\
\hline
\end{tabular}

Note. $\checkmark=$ dynamic domain predictive of reoffending outcome. $\mathrm{X}=$ dynamic domain not predictive of reoffending outcome. 


\section{Study 1 Discussion}

This study was one of the first to examine changes in dynamic risk factors and strength factors over time for both men and women, and examine how these changes were related to various criminal outcomes. Previous multi-wave studies incorporating three or more timepoints to assess change in dynamic risk factors over time have focused predominately on men (e.g., Brown et al., 2009; Hanby, 2013; Jones et al., 2010) with one exception, Greiner et al. (2015) who looked at women. To date, only two studies have assessed the dynamic nature of strengths and how these changes are related to offending outcomes (Hanby, 2013; Lloyd, 2015). Both Hanby (2013) and Lloyd (2015) assessed changes in strengths measured using the Dynamic Risk Assessment of Offender Re-entry (DRAOR; Serin, 2007). However, they used different approaches and found different results. First, Hanby (2013) used Cox regression to assess the incremental change among the various twelve timepoints and found that although strengths increased over time, these scores over time were not predictive of reconvictions. However, Lloyd (2015) assessed change in strengths over time using a discrete-time hazard model and found that incorporating change in strength scores added to the predictive accuracy of assessing initial strengths scores or static scores alone. Overall, given the lack of research assessing the gender differences in changes on dynamic risk and strength scores and the predictive nature of these change scores, the current study aimed to address this gap. Specifically, the two goals of the current study were to: 1) examine how total dynamic risk and strength scores change over time for men and women, and 2) examine how these changes in dynamic risk and strength scores predict criminal outcomes, including any new charges, any violent charges, technical violations, and any negative outcome. 


\section{Patterns of Change in SPIn-Assessed Total Dynamic Risk Scores}

The first research question examined how SPIn-assessed dynamic risk factors change over time and whether these changes vary as a function of gender. Based on previous research it was expected that there would be a general decrease in total dynamic risk scores over time for both men and women. To assess this hypothesis, 11,953 men and 2,877 women with 3 or more full SPIn assessments over the span of 30 months were included in analyses. To assess patterns of change, HLGM was used for the sample of men and women independently, and static risk score, age, and Indigenous status were included as covariates into the unconditional growth model to explain variability in initial and change scores. In terms of initial dynamic risk scores, men and women tended to start at similar scores, which were related to static risk scores (i.e., those who had higher static scores were higher on initial dynamic risk score). Although not as influential as static risk, it was found that age influenced initial dynamic risk scores for men; that is, men who were younger had slightly higher initial dynamic risk scores than men who were older.

In terms of change scores, the results indicated that total dynamic risk scores change over time in a similar pattern and rate for both men and women, and the rate of change over time was minimal for both genders. Static risk total score did not influence the change in dynamic risk scores over time for men. In contrast, for women, change in dynamic risk scores over time were significantly influenced by total static risk score whereby those who had a higher static risk score (in addition to having a higher initial dynamic risk score) had scores on dynamic risk that decreased faster over time than those 
who had lower static risk scores. In fact, women who had lower static risk scores had low initial scores on dynamic risk, and these scores actually slightly increased over time.

There may be a number of reasons for this counter-intuitive finding. First, it is important to recognize that the majority of the women included in the analyses were low risk caseS (e.g., 57.6\% scored low on static risk). Thus, given that the average static risk score for the full sample is already quite low, those who scored one standard deviation below the mean would be considered very low risk offenders (on static risk). As such, there is a possible floor effect whereby the ability of the SPIn to measure changes below this point may be extremely difficult and any slight deviation (e.g., increase) in dynamic risk scores for this extremely low risk group may result in what appears as the offender getting riskier over time.

This finding is consistent with past research indicating that while the majority of low risk offenders do not change, when they do, it is often an increase in risk rather than a decrease. For example, Cohen, Lowenkamp, and Van Benschoten (2016) examined changes in risk classification levels for offenders across different risk levels. Notably, among 21,732 offenders who were initially deemed low risk, $92 \%$ were found to have no change in classification level over time. Although it was not possible for offenders in the low risk category to decrease any further (low risk is the lowest classification they could have received), it was interesting to see that $8 \%$ of the sample increased in risk level over time. When aggregating all risk levels together $(N=64,716)$, it was found that $82 \%$ of offenders remained in the same risk level classification over time, $10 \%$ decreased in risk over time, and $8 \%$ increased in risk over time. The majority of the Cohen et al. (2016) study was male $(82 \%)$, and thus this floor effect may be even more problematic for 
women given that more women tend to be low risk than men. Regardless, in the current study, when the findings were taken together, the overall trend indicated a decrease in dynamic risk over time for women.

For men, the biggest difference in rates of change on total dynamic risk scores was between Indigenous and non-Indigenous men. Although both had similar initial scores, non-Indigenous men had a slightly faster rate of change (decrease in scores) over time. While the overall change trends were anticipated, the rate of change was expected to be faster than what was evidenced in the models. Given the total dynamic risk scores can range considerably, it was expected that with every month that passed, the scores would change more than a fraction of a point. However, it is important to remember that model results also demonstrated that there was unexplained variance in the model, indicating that additional variables may be needed, or that model specification can be improved, in order to explain the remaining variance.

It is also important to consider that the change over time may be a bi-product of raters having more information about the offender and having a better sense of an offender's true score on the risk assessment as time progresses. For instance, it is plausible that at the start of supervision, probation officers may have very limited information for rating all the SPIn items, and thus choose to rate cautiously until more information is gathered (Lloyd, 2015). This would indicate that change depicted in the findings is not reflective of true offender change, but rather a more accurate assessment rating of the offender's risks. Jones and colleagues (2010) have also suggested that raters' expectations of offenders' level of functioning may change as time progresses, thus how the items are rated may fluctuate, which could also impede the detection of genuine 
offender change. However, based on the results from the assessment of measurement invariance for the current study, this is not the case (i.e., the same construct was found to be measured at each assessment period in the current study).

\section{Patterns of Change in SPIn-Assessed Total Dynamic Strength Scores}

The second research question examined how SPIn-assessed dynamic strength factors change over time and whether these changes vary as a function of gender. Based on previous research (e.g., Hanby, 2013; Lloyd, 2015) it was expected that there would be a general increase in total dynamic strength scores over time for both men and women. The results indicated that total dynamic strength scores increased over time in a similar pattern and rate for both men and women; however, the rate of change over time was minimal for both genders. For women, both age and static risk score significantly influenced scores on initial dynamic strength scores. However, age, Indigenous status, and static risk scores all significantly influenced initial total dynamic strength scores for the men. The most influential covariate for the men was Indigenous status, where Indigenous men scored higher on initial dynamic strengths than non-Indigenous men.

In terms of change scores, static risk total score, age, and Indigenous status, each explained significant variability in changes in dynamic strength scores over time for both men and women. Notably, Indigenous status explained the most variability in change in dynamic strength scores, where for both men and women, the rate of change in dynamic strength scores (increase over time) was slightly faster for both non-Indigenous men and women. While the overall change trends were anticipated, the rate of change was expected to be faster than what was evidenced in the models. 
The reasons for such gradual change for both total risk and strength scores could be due to a number of reasons, including the fact that a high proportion of the sample was made up of low-risk men and women. Therefore, decreases in risk scores would be relatively minimal (and potentially so would increases in strength scores). However, it could also be the case that probation officers are merely using scores from previous assessments to pre-populate SPIn scores input into the computerized SPIn software. Through personal communications with the Programs and Policy Development unit from Alberta Justice and Solicitor General (January 28, 2020), it was indicated that there is an option by which probation officers can input scores from the previous assessment and can then adjust specific scores as necessary. Given that probation officers have caseloads that are quite demanding, it would be no surprise if this was used as the default. Specifically, research conducted by Bonta, Rugge, Bourgon, and Wanamaker (2019) found that the average caseload for probation officers in Alberta was 71 clients. Although this average caseload is comparable to the average caseload found across three other Canadian provinces, which was 65 clients (e.g., British Columbia, Saskatchewan, and Prince Edward Island; Bonta et al., 2011; Wormith, Ferguson, \& Bonta, 2013), this requires a large amount of resources. Given the large caseloads and the fact that the SPIn is comprised of 90 items to assess, it is probable that the score pre-population function would be used frequently. While this function would be useful for saving time for the probation officer, it increases the likelihood of maintaining similar scores across timepoints (e.g., previous scores influence current assessment scores).

Nonetheless, the current study found results that were comparable to other studies assessing offender change in dynamic risk and dynamic strength scores. For men, the 
results found in this study were similar to results found by Hanby (2013) and Lloyd (2015) the only other study to examine patterns of change in both dynamic risk and strength scores over time. Hanby (2013) found that over time, risk scores tended to decrease while at the same time, strength scores tended to increase as survival time increased. For the women, the results were similar to those found by Greiner and colleagues (2015), which was the only study to look exclusively at change scores for women, who found that dynamic risk scores decreased over time.

Upon examining the pseudo $R^{2}$ results, a large proportion of variance was explained by the variables included in the models — static risk, age, and Indigenous status. However, there may be additional variables that are pertinent to explaining the remaining variability in both the dynamic risk and strength models, for both genders. A greater deal of variation was explained in the dynamic risk model than in the strength model for men and women, indicating that there may be additional variables that should be included in these models, such as program information.

\section{Prediction Results for Men and Women}

The second goal of Study 1 was to examine the predictive ability of change in dynamic risk and strength scores. Specifically, the third research question examined whether the rate of change in SPIn-assessed total dynamic risk and strength scores predicted reoffending for both men and women. For men, both the initial dynamic risk score and the change over time in dynamic risk was predictive of all four criminal outcomes, which included any negative outcome, any new charges, new violent charges, and technical violations. However, the change over time in dynamic risk was a stronger predictor of each of the four criminal outcomes. These results align with the results of 
Brown et al. (2009), Howard and Dixon (2013), Jones et al. (2010), and Lloyd (2015) who found that dynamic re-assessment information was predictive of recidivism over and above the predictive ability of initial dynamic risk scores.

For women, the initial dynamic risk score was predictive of all four outcomes, similar to the men. However, the change in dynamic risk scores was not predictive of violent charges but was predictive of the other three criminal outcomes (any negative outcome, any new charges, and technical violations). With the exception of violent charges for women, the change in risk scores over time were more predictive of reoffending outcomes than initial dynamic risk scores. For women, the change in risk scores may not be predictive of violent charges because of the low base rates of violent charges (e.g., 292 women or 10.1\% were charged with a violent offence), coupled with the minimal change in scores over time. Nonetheless, these results were similar to those found by Greiner et al. (2015), who found that re-assessment information of dynamic risk was more predictive of reoffending than initial assessments for women. However, the previous studies did not look explicitly at the relationship between the rate of change in dynamic risk scores and reoffending outcomes, but rather examined the relationship between incremental change and reoffending, or compared the predictive accuracy of proximal versus distal risk assessment information.

Results also indicated that for both men and women, the predictive ability of initial dynamic risk and change in dynamic risk scores was strongest for technical violations, followed by any negative outcome (which includes the technical violations outcome). As discussed in Lloyd (2015), dynamic risk scores may be most predictive of technical violations because the SPIn assessments are rated by probation officers, the 
same individuals who would support a breach of an offender (i.e., a technical violation). In other words, the supervising officer who is responsible for assessing an offender is, in most cases, the same individual responsible for initiating a breach of that offender. Given that it is unknown how long a supervising officer was considering to support or initiate a technical violation for a client breaching his or her conditions, risk assessment ratings could potentially have been influenced by rater knowledge of upcoming technical violations (Lloyd, 2015). However, because this knowledge is most likely to influence the more proximal SPIn assessment ratings, and since the change scores used in the current study are reflective of an average change rate of SPIn scores over time, it is unlikely that this is the sole reason for the relationship between change scores and technical violations. Furthermore, the initial SPIn scores were also most predictive of technical violations, indicating that despite the potential argument that more proximal ratings are being influenced by the knowledge of an upcoming breach, there is evidence to suggest that SPIn assessments (and re-assessments) are accurately predicting technical violation outcomes. This also demonstrates that the decision to support or initiate a breach for an offender is at least partially reflected by information rated by the SPIn. In contrast, for strengths, there were some differences between genders. For men, initial strength scores were predictive of any new charges and technical violations, although the magnitude of these effects were extremely small. Specifically, men with higher initial strength scores were less likely to be charged with a new offence or a technical violation in compared to those with lower initial strength scores. Change in strength scores were only predictive of technical violations, whereby men with strengths scores that increased over time were less likely to commit a technical violation. For 
women, initial strength scores were predictive of any negative outcome, violent charges, and technical violations, but not predictive of any new charges. The magnitude of these effects was quite small. Similar to the men, the only criminal outcome that change in strength scores predicted was technical violations. These findings demonstrate the importance of considering strengths, particularly when predicting more immediate reoffending outcomes (such as technical violations).

It is important to note that for the men, the prediction analyses could only be conducted on a subsample of men who had change parameters (slopes and intercepts) on dynamic risk and dynamic strength total scores. This was due to the limited memory of the statistical program, which would not allow the change parameters to be saved for the entire sample of men given the large sample size and large number of observations. As such, a random sample of men were chosen and upon comparing to the full sample of men, there were no statistically significant differences between groups (thus the random sample was representative of the full sample of men included in the first stage of the twostage HLM approach).

Overall, these results highlight the importance of examining change in scores over time. In most cases, the change in scores (both dynamic risk and strengths) was a stronger predictor of various criminal outcomes, in comparison to the initial assessment score on these domains. These results support the findings from other multi-wave studies, including Brown et al. (2009), Greiner et al. (2015), Howard and Dixon (2013), Jones et al. (2010), and Lloyd (2015), where change scores improved prediction of offending outcomes over the use of initial dynamic risk score. These results also demonstrate some important gender considerations. Firstly, while initial assessment scores and change 
patterns are relatively similar for both men and women, there were some differences in the predictive ability of the two total scores in predicting criminal outcomes. For instance, although the dynamic risk initial scores and change over time were both predictive of criminal outcomes for men and women, for most of the outcomes, the predictive ability was stronger for men. In contrast, the strength scores (both initial and change over time), appeared to be a stronger predictor of criminal outcomes for women than for men. Notably, for both men and women, the only outcome where change in strength scores over time was predictive was for technical violations, and the magnitude was stronger for women than men. For men initial strength scores were predictive of both technical violations and any new charges, whereas for women, initial strength scores were predictive of technical violations, any violent charges, and any negative outcome (but not any new charges).

\section{Implications}

True dynamic factors. According to Brown et al. (2009), to be considered truly dynamic, a factor should demonstrate significant change over time and should be significantly related to the prediction of recidivism. Although this research did not assess change in SPIn dynamic domains, the results indicated that the total dynamic risk score demonstrated significant change over time and significantly predicted recidivismspecifically new charges, violent charges, technical violations, and any negative outcome. There was also evidence that total dynamic strength scores significantly changed over time, and predicted technical violations. As such, evidence supports the use of the SPIn total dynamic risk and total dynamic strength scores to inform case planning. 
Importance of strengths. Although previous prospective research has found that strengths measured on the SPIn add incrementally to risk in the prediction of recidivism (Jones et al., 2015), this was the first study to use a multi-wave longitudinal design to examine the predictability of the initial assessment total strength scores and the change in total strength score over time. Overall, results indicated that initial strength scores were predictive of technical violations, and any new charges for men, and predictive of any negative outcome, technical violations, and violent charges, for women. For both men and women changes in strengths were only predictive of technical violations. As such, this may have practical implications on how to approach case management practices and community programming. Specifically, offering community strengths-based programs and strengths-based case management planning at the start of community supervision may assist with reducing the number of technical violations for men and women. Notably, while the results supported the predictive nature of strengths for men and women, strengths were stronger predictors for women. In comparison, dynamic risk scores were more predictive for men. This highlights the importance of providing strengths-based interventions specifically for women, to assist with reducing reoffending-especially technical violations.

There is evidence to suggest that incorporating a strengths-based approach in treatment while under community supervision improves psychological wellbeing, likelihood of remaining in and participating in treatment, and reduces recidivism for women (Messina, Calhoun, \& Warda, 2012; Messina, Grella, Cartier, \& Torres, 2010). As discussed in Fedock and Covington (2018), there are five key factors that are especially important for women re-entering the community and each of these factors 
requires the use of strengths-based approaches to address them. These factors include finding shelter, finding stable employment and financial stability, rebuilding relationships with others, developing a sense of community belonging, and building self-esteem (O'Brien, 2001). In addition, the interaction between probation officer and client should also be rooted in a strengths-based approach. For instance, Morash (2010) found that supervision practices incorporating positive, strength-building approaches, such as motivational interviewing, can lead to positive changes - such as feelings of empowerment and increased self-esteem (Fedock \& Covington, 2018), which can assist with improving the lives of women currently involved in the criminal justice system.

Intersectionality. An issue that warrants discussion is the importance of intersectionality. Examining the intersection of gender and other potentially discriminating factors (such as race and sexual preference), is also important to consider for future studies as research suggests that these additional factors may assist in advancing our understanding of the relationship between gender and crime (Baca Zinn \& Thornton Dill, 1996; Burgess-Proctor, 2006; Daly \& Tonry, 1997).

Traditional feminist criminologists have focused predominately on White, middle-class women, and as such, contemporary feminist criminologists have started to advance the field by recognizing that the lived experiences of minority women are very different (Burgess-Proctor, 2006). The intersectionality approach recognizes that there are multiple forms of oppression and inequality other than inequality based purely on gender. Specifically, aside from gender, women can face multidimensional inequalities on account of race, social class, sexuality, socio-economic status, and other sociallyconstructed discriminations - such as individuals involved in the criminal justice system 
(Anderson \& Collins, 2004). In Canada, an important area where the intersection of gender and ethnicity is rarely investigated, is gender and Indigenous status among those involved in the criminal justice system. Specifically, although correctional research has been conducted on Indigenous Peoples and on gender differences, rarely has the intersection of the two been examined (Bartels, 2012).

Indigeneity. Indigenous Peoples are vastly overrepresented in the criminal justice system in Canada. Although for the purpose of this study Indigenous men and Indigenous women were two subgroups included in analyses, it is important to acknowledge the unique histories and diversity among Indigenous cultures; that is, there are distinct cultural, geographical, and historical differences among First Nations, Metis, and Inuit peoples. In the current study, at the time of the initial SPIn Full Assessment, 16.6\% of men $(n=1,988 / 11,953)$, and $28.8 \%$ of women $(n=829 / 2,877)$ identified as Indigenous, in comparison to $6.5 \%$ of the general population in Alberta who identify as Indigenous (Malakieh, 2018). This overrepresentation in the criminal justice system may be due to the unique needs of Indigenous men and women — such as, increased substance use, mental health issues, residential school experiences, intergenerational trauma, welfare involvement, family history of suicide, and extreme poverty and poor living conditions on reservations (Ellerby \& MacPherson, 2002; Office of the Correctional Investigator, 2015; Shepherd, Adams, McEntyre, \& Walker, 2014). Although several of these risks and needs may also be relevant for non-Indigenous men and women, the reason behind these needs and risk factors may differ, or the operationalization of these concepts may be different (Helmus, Babchishin, \& Blais, 2012; Wilson \& Gutierrez, 2014). Therefore, 
the extent to which general risk assessments can be used with Indigenous Peoples involved in the criminal justice system requires further attention.

As such, it is important to consider how well items on the SPIn capture the unique circumstances around the various risk and need factors for Indigenous men and women. While it was beyond the scope of the current study, Indigenous status was included as a covariate - given the high proportion of Indigenous men and women in the sample. Results suggested that, overall, rate of change on both total dynamic risk score and total dynamic strength score was minimal, with slower rates of change on scores than nonIndigenous men and women (with the exception of total dynamic risk scores for women). Future research is encouraged to examine how Indigenous men and Indigenous women change over time on both dynamic risks and strengths that incorporates the examination of more culturally-relevant factors.

Gender identity considerations. A limitation of the current study is that it focused solely on cis men and cis women (that is, those who identify with the gender that they were assigned at birth). In line with intersectionality, additional research is needed focusing on various gender-identities and expressions (as opposed to the archaic dichotomous categorization of man or woman). Research and data on people who identify as gender non-conforming and transgender (GNCT) within the criminal justice system are quite limited (Woods, 2017) and the research that does exist is predominately conducted on adolescent samples (Dennis, 2014). Sexual orientation, which is often considered in connection with gender-identity is also highly under-researched in the criminal justice field (this includes individuals who are lesbian, gay, bisexual, and questioning-LGBTQ+). Some reasons for the limited research in these various 
subgroups of those involved in the criminal justice system may, in part, be a reflection of sample size. Nonetheless, it is important to consider the unique experiences of LGBTQ+ individuals and how these experiences, both on their own and coupled with other forms of discrimination, may influence their pathways into the criminal justice system, and thus, LGBTQ+ individuals may have unique needs that must be captured by risk assessment protocols and targeted in programming.

Other change-related research. This study was the first to use SPIn data to examine how total dynamic risk and strength scores change over time for both men and women. More research is needed assessing change in scores over time and how these changes are related to various criminal outcomes, including reconvictions and sexual offences. Given that the current study was not able to identify whether the most proximal assessment was the most predictive of future offending, additional research is needed to assess whether the last assessment prior to reoffending is the most predictive or if there is a certain point in time where assessments no longer add incrementally to the initial change score (e.g., analyses using Cox regression survival analyses can help pinpoint incremental change between timepoints; Brown et al., 2009; Hanby, 2013). There are benefits to assessing incremental change in scores - namely, the ability to see how factors change from one timepoint to another, and the ability to determine which factors change more rapidly (e.g., acute dynamic factors) versus those that change more gradually (e.g., stable dynamic factors), which is useful for informing treatment and case planning. As such, additional research is also needed examining changes in the specific dynamic domains (both risks and strengths) to see if there are specific areas that are most predictive of reoffending outcomes. There may be certain factors that are more predictive 
for one gender over the other, which can assist with informing treatment and case management planning.

Additional research is also needed assessing change in dynamic risk and strengths using the Two-Stage Hierarchical Linear Modeling approach that was used in the current study. As discussed in Yang et al. (2017) this approach is not commonly used in conjunction with criminal justice and risk assessment data, but is used frequently in epidemiological research. Given that the current study was one of the first to use this approach to assess patterns of change and use that change to predict reoffending outcomes, future research is encouraged to continue to use this advanced statistical approach. The two-stage HLM has several benefits over the use of Cox regression with time-dependent covariates including the ability to examine multiple reoffending outcomes, such as frequency of recidivism, and the ability to simultaneously examine how individuals change or how patterns of change differ for individuals, which cannot be assessed using Cox regression.

Finally, upon conducting an exploratory factor analysis on the 15 dynamic domains to examine measurement invariance, it was found that a three-factor solution fit the data best for both the men and the women. The three factors were dynamic strengths, intrapersonal risk, and interpersonal/stability risk. The assumption of measurement invariance of the three-factor structure was met only for women, whereas for men, only the two-factor solution used in the current study (dynamic strengths, dynamic risks) was found to meet the measurement invariance assumption. As this was the first study to assess the factor structure of the SPIn, future research is encouraged to examine whether the best fitting factor structure of the SPIn is different for men and women. Additional 
research examining the factor structure of the SPIn is needed, particularly using itemlevel data.

\section{Limitations}

Loss of data and generalizability concerns. To assess change over time, data on at least three timepoints were required. Because of this requirement, there was a loss of almost 13,000 cases who either had no completed SPIn Full Assessment or who only had one or two full assessments. This was either due to failure prior to the completion of three assessments (e.g., reoffending early on while on supervision) or because data was missing on assessment information. However, as Lloyd (2015) indicates, this is a fundamental challenge of assessing change among justice-involved populations. As such, this research is limited to those who are serving time in the community on a provincial sentence in Alberta. These results cannot be generalized to offenders serving a Federal sentence in Canada, as they are typically higher risk and serving longer sentences for more serious offences, and cannot be generalized to offenders serving time in the community in other provincial jurisdictions. Offenders in each province are unique, both in terms of the types of offenders serving time in the community, but also in terms of the treatment they receive and the policies guiding offender rehabilitation and case management.

In addition, the sample was comprised predominately of low-risk men and women. Low risk cases are not expected to exhibit much change over time-primarily because they exhibit fewer needs and more strengths than high risk cases. Upon reviewing the literature, Bonta (2009) found that treating high risk cases resulted in an $11 \%$ reduction in recidivism whereas for low risk cases, treatment resulted in a $3 \%$ 
reduction in recidivism. Low risk cases should, thus, receive minimal services and limited contact with probation officers, as correctional interventions may be ineffective and may actually increase risk of reoffending (Cohen, Cook, \& Lowenkamp, 2016; Lowenkamp, Latessa, \& Holsinger, 2006). As such, given that changes in low risk men and women are found to be minimal, and given that the sample was predominately low risk (that is, limited variability in risk scores in the sample), the changes found in the current study align with previous research. Future research is encouraged to examine change related to higher-risk cases to attain a better understanding of how dynamic risk and dynamic strengths change over time among each level of offender classification.

Calibration of the SPIn. Given that risk assessment tools differ among provinces, comparability of rate of change, even among classification risk levels poses challenges. For instance, in Alberta the median score on the SPIn is considered low risk, however, the median score on the Saskatchewan Primary Risk Assessment (SPRA; Saskatchewan Ministry of Corrections, 2009) is considered moderate risk (Bourgon, Mugford, Hanson, \& Coligado, 2018). Moreover, the recidivism rates for those deemed high risk on the SPIn do not align with the recidivism rates for those deemed high risk on the Level of Service Inventory-Ontario Revision (LSI-OR; Andrews, Bonta, \& Wormith, 1995), indicating variability in what is meant by high risk among jurisdictions (Bourgon et al., 2018). Given the variability between the SPIn and other risk assessment tools, future research should further examine the calibration of the SPIn. Classification levels can have many implications on justice-involved populations in terms of frequency of contact and access to programming. Ensuring that men and women classified as low risk are in fact, low risk to reoffend is important for ensuring successful rehabilitation efforts. 
Psychometric data and ability to assess change. Another limitation is the lack of psychometric data available for the SPIn. While there has been some research conducted on the validity and internal consistency of the SPIn, which have yielded promising results, no research has been conducted on the interrater reliability of the tool (Jones \& Robinson, 2018). Given that there is no information on whether scores are being assessed in the same way across probation officers, this may have affected the change results. Additionally, whether the SPIn is discriminant (that is, whether the SPIn can adequately classify offenders to low, moderate, or high risk categories) is largely unknown; although research examining the predictability of the tool has indicated that offenders classified as high risk are in fact more likely to reoffend, than those classified as low or moderate risk (Jones et al., 2015; Jones \& Robinson, 2017a, 2017b). More research is needed looking at the psychometric properties of the SPIn, especially among different subgroups of offenders.

Due to the minimal amount of change over time for both the total dynamic risk and strength scores, another potential concern is the tool's ability to measure true offender change. The current study was the first to assess change in SPIn scores. As a first step, measurement invariance was assessed to examine if exhibited change over time was in fact true change or if it was due to issues with the assessment's ability to measure certain constructs over time. It was found that over time, the SPIn is measuring what it is intending to measure, however, this analysis was done at the domain level, and should also be examined at the item level.

Sensitivity of item scores. Importantly, some SPIn items are measured on a poled Likert scale with the scores ranging from 0 to 2 for strength and 0 to 2 for risk; although 
for items assessing both risk and strength, if rated as 1 or 2 on risk, the corresponding score for strength would be 0 , and vice versa. A score of 0 typically indicates "none" or "neutral", where as a score of 1 typically indicates that the item is "sometimes" or "somewhat" present, and finally a rating of 2 indicates that an item is "always present". It may be that the SPIn is not sensitive enough to capture change or that the distinction between a score of "1" and " 2 " are not clear (or the probation officer does not have enough information to distinguish between a score of 1 and a score of 2 on some items). Similarly, given that the SPIn is comprised of 90 items, it is entirely possible that there is not enough discrimination between items, thus affecting the tools sensitivity to detect change. As such, future research is encouraged to run exploratory and confirmatory factor analyses to examine the factor structure of the SPIn, as well as identify the items that are deemed to explain the most variability in domain scores (i.e., highest loading factors).

Regardless, to improve risk assessment accuracy, those who use the SPIn are provided with a detailed administration guideline manual that assists with scoring the risk assessment with offenders; raters use a semi-structured interview, along with file review information, to assist with gathering accurate and detailed information on the offender. Also, in contrast to other risk assessment tools who score items dichotomously (such as the Inventory of Offender Risk, Needs, and Strengths [IORNS]; Miller, 2006), having the SPIn items scored on a Likert scale, allows for more item variability. Orbis Partners suggest that scoring items on Likert-type scales enhances measurement sensitivity and increases a tools ability to quantify incremental change (Jones \& Robinson, 2018). However, it is possible that additional scoring options (such as using a larger Likert 
scale) would enhance the sensitivity of the tool, as it would add increased variability in scores on the various items.

Lack of program information. One final limitation worth discussing is the lack of data on program information for each client. Because there was no information on program completion, the reason behind offender change could not be examined. It would be worthwhile for future research to examine change in relation to completed programming to see how much correctional programming is influencing rates of change in dynamic risk and strength scores over time. While programming is often contracted out to the communities, rather than conducted in-house (personal communications with the Programs and Policy Development unit from Alberta Justice and Solicitor General on January 28, 2020), there may be differences among jurisdictions due to resources and client needs. As such, one area of future research would be to examine if there are jurisdictional differences in offender change on total dynamic risk and strength scores. For example, conducting a three-level HLGM, with jurisdiction as the third level, or incorporating programming information into analyses.

\section{Summary}

Despite the aforementioned limitations and areas for future research, the current study adds to the literature on change in risk and strength scores in several ways. First, this study was the first to use a rigorous statistical approach to assess change and prediction of SPIn-assessed dynamic risk and strength total scores. Specifically, the twostage hierarchical linear modeling approach was utilized, which has commonly been used in epidemiological studies. This approach allows for the direct assessment of how change parameters (intercept and slope) derived from hierarchical linear growth modeling 
predicted various criminal outcomes. This was also the first study to directly compare gender differences and similarities in changes in scores over time, and only the third multi-wave longitudinal design to incorporate the assessment of change scores in strengths over time. Overall, results suggested that for both men and women, dynamic risk scores decreased over time, as success in the community continued. In fact, dynamic risk scores for men and women that decreased at faster rates over time were less likely to reoffend across all outcomes - technical violations, new charges, violent charges, and any negative outcome (with the exception of violent charges for women). For both men and women, strengths increased over time, as success in the community continued, and change scores were predictive of technical violations only (for both genders). Results demonstrated the utility of re-assessing dynamic risk and strength scores over time, as well as highlighted that men and women are more similar than different. 


\section{Chapter 4}

\section{Study 2: Building a Holistic Typology to Inform Service Delivery for Men and Women on Community Supervision}

Offender typology research has been prominent in both mainstream and genderresponsive correctional research, whereby offenders are grouped based on commonly shared characteristics. Often these typologies are based on the clustering of various risk factors thought to be common among a subset of offenders. According to Jones and Harris (1999), there are four reasons why classifying offenders into various typologies is beneficial: 1) it can help build our understanding of why people engage in criminal activity, 2) it can aid in our approach to treatment and intervention protocols to improve responsivity, 3) it can help guide case management of offenders, and 4) it can help determine who are more likely to reoffend.

Whether a typology study can inform one of these four areas depends upon the variables included and the underlying purpose of the study. Studies have used numerous statistical techniques to develop these typologies (e.g., multidimensional scaling, path analysis, latent class analysis, cluster analysis), which has increased the variability in the number of identified subtypes of offenders. There is also variability in terms of how typologies are derived based on theories of criminal behaviour. As described by Greiner (2013), mainstream correctional researchers (e.g., gender-neutral researchers) have typically derived typologies using reoffence rates, risk/need factors, and offender characteristics rather than theory and tend to fall into one of two categories - trajectorybased typologies and risk/need based typologies. In contrast, gender-responsive researchers have typically relied upon theoretically-derived pathways to criminal 
behaviour, including factors that have little quantitative empirical support (due to lack of research) in predicting offending — such as child abuse and victimization.

Although typology research has been prominent in the criminal justice field, there are several areas that have not yet been explored, or that are missing from typological studies. First, while gender-neutral and gender-responsive researchers have assessed offender typologies, few studies have incorporated both gender-neutral and genderresponsive variables in the typology building process. Second, these studies have typically focused on one gender-failing to identify potential gender differences in how certain variables may cluster together. Third, virtually no typology or strengths-based studies have examined how strengths may aid in the development of offender-based typologies. Finally, the majority of typology studies have been cross-sectional in design, focusing solely on one timepoint, and do not examine how these classes remain stable or change over time. As such, the current study will combine tenets of the gender-neutral, gender-responsive, and strengths-based perspectives to examine typologies that emerge for men and women on community supervision and whether these typologies remain stable over time. Trajectory-based typology research and risk/need based typology research informing the current study is described in detail.

\section{Trajectory-Based Typology Research}

This type of offender research examines the development of criminal behaviour over time (e.g., how it increases, decreases, stays the same) and focused predominately on adolescence. Moffitt (1993) conducted one of the earliest and most influential trajectory studies. To summarize, Moffitt argues that there are two main types of offenders (for males and females alike): adolescent limited (AL) offenders and life- 
course persistent (LCP) offenders. AL offenders begin demonstrating antisocial behaviour in adolescence and engage in minimal criminal activity, but then refrain from criminal behaviour upon entering adulthood. In comparison, the LCP offenders begin to demonstrate antisocial behaviour in childhood and continue into adulthood, gradually engaging in more severe criminal activity as time passes. Although there has been a wide range of empirical support for Moffitt's developmental dual taxonomy theory of criminal behaviour, more recent research suggests that a dual taxonomy of offending may not be capturing individual variation in offending (Salvatore, Taniguchi, \& Welsh, 2012).

Piquero (2008) conducted a review of 80 studies that used trajectory analyses and concluded that three to five groups consistently emerged. Notably, most of these studies looked solely at male offending patterns, and only a few incorporated samples of females (e.g., Chung, Hill, Hawkins, Gilchrist, \& Nagin 2002). Studies that have included justiceinvolved females tended to use small sample sizes and developed trajectories using behaviours and situations more relevant to males (i.e., physical aggression versus indirect aggression; Fontaine, Carbonneau, Vitaro, Barker, \& Tremblay, 2009). Fontaine and colleagues (2009) conducted a literature review summarizing 46 studies. Results indicated that while females followed similar offending trajectories as males, there was an additional trajectory unique to females, the adolescent-delayed-onset trajectory, where females with childhood risk factors had a delayed onset of criminal behaviour, occurring later in adolescence. One limitation of this research, however, is that studies tend to focus on childhood and adolescent trajectories (Fontaine et al., 2009). 


\section{Gender-Neutral Risk and Need Based Typology Research}

A second type of typology research that is prevalent among mainstream correctional researchers is risk or need based typologies (Greiner, 2013). This typology research groups offenders based on their risk factors, which can help inform effective correctional treatment interventions and aid in our understanding of how various risk/need factors may operate together. Most studies have been conducted with samples of male youth (e.g., Simourd, Hoge, Andrews, \& Leshied, 1994), although studies have begun to use youth samples comprised of both males and females (e.g., Dembo \& Schmeidler, 2003; Onifade, Nyandoro, Davidson, \& Campbell, 2010).

Generally, these studies have found between four and five typologies of justiceinvolved youth. Each of these studies have found a general low-risk subgroup and a general high-risk subgroup, as well as two or three subgroups defined by specific dynamic risk factors, such as personality deficits or substance use deficits. These studies typically do not include hypothesized, gender-responsive need factors, such as victimization or mental health issues, but include traditional risk factors (i.e., the Central Eight). As such, all relevant risk factors for both females and males may not be captured.

To account for this limitation, Brennan, Breitenbach, and Dieterich (2008) included both gender-neutral (i.e., substance use, educational issues, aggression, and family issues) and hypothesized gender-responsive risk factors (i.e., physical/sexual abuse, promiscuity, socioeconomic status) in a multiple cluster analysis. Using a sample of 1,572 juvenile offenders ( $72 \%$ male) from three jurisdictions in America (a Midwestern state, a Western state, and a Southern state), Brennan and colleagues (2008) found several clusters that emerged: 1) Internalizing youth A, 2) Socially deprived, 3) 
Low control A, 4) Normal “accidental/situational” delinquents, 5) Internalizing youth B,

6) Low control B, and 7) Normative delinquency (see Table 28 for a description of each).

Table 28

Summary of Brennan et al. (2008) Typologies

\begin{tabular}{|c|c|c|}
\hline Typology & Description $^{1}$ & $N$ \\
\hline Internalizing Youth A & Withdrawn, abused and rejected delinquents & 83 \\
\hline Socially Deprived & $\begin{array}{l}\text { Socially deprived delinquents from lower } \\
\text { socioeconomic statuses }\end{array}$ & 103 \\
\hline Low Control A & $\begin{array}{l}\text { Versatile, impulsive, low empathy, manipulative, } \\
\text { negative school experiences and pro-criminal peers }\end{array}$ & 85 \\
\hline $\begin{array}{l}\text { Normal "accidental/ } \\
\text { situational" } \\
\text { delinquents }\end{array}$ & $\begin{array}{l}\text { Youth who display limited risk factors, lower } \\
\text { number of adjudications, and lower age at first } \\
\text { adjudication }\end{array}$ & 151 \\
\hline Internalizing Youth B & $\begin{array}{l}\text { Similar to Internalizing Youth A, but have } \\
\text { significantly higher mean number of violent } \\
\text { charges and do not come from abusing or } \\
\text { neglectful households }\end{array}$ & 197 \\
\hline Low Control B & $\begin{array}{l}\text { Delinquents with early onset and versatile } \\
\text { offenders with multiple risk factors; more extreme } \\
\text { than the Low Control A group }\end{array}$ & 146 \\
\hline $\begin{array}{l}\text { Normative } \\
\text { Delinquency }\end{array}$ & $\begin{array}{l}\text { Youth with few risk factors, but tend to have pro- } \\
\text { criminal peers, engage in substance use, and are } \\
\text { promiscuous }\end{array}$ & 130 \\
\hline
\end{tabular}

Note. ${ }^{1}$ See Brennan et al. (2008) for a complete list of variables that fall under each cluster. Approximately $43 \%(n=677)$ of the sample was deemed unclassified because they were hybrid cases or outliers.

Notably, $43 \%$ of the sample was deemed to be unclassified — due to being a hybrid case or an outlier $(n=677)$. As such, a large proportion of the sample remained absent from the seven clusters. Clusters were also not disaggregated by gender, and the 
number of females who belonged to each cluster, and the unclassified group, was not discussed.

\section{Justice-Involved Female Typology Research}

Upon review of the literature, only one dynamic risk typology study operating through a gender-informed lens has been completed (Dembo et al., 2008) that includes both traditional, gender-neutral risk factors (e.g., peer relationships, education), as well as gender-responsive risk factors over time (i.e., mental health issues, maltreatment). A latent transition analysis (LTA) was conducted on a sample of 137 youth (52\% male) from Florida, USA. Latent profile analysis (LPA) was conducted at baseline and 1 year later. Then an LTA was conducted to examine if the typologies were stable over time and whether there were changes in typological membership. Overall, four risk areas were examined: family relationships, peer relationships, mental health experiences, and education. Two typologies emerged at both timepoints-one scoring high on all risk categories and one scoring low on all risk categories.

Upon examining the changes in typologies at each timepoint, two classes of latent transitions were found: a class demonstrating change (i.e., changes from low to high factor category or from high to low factor category from Time 1 to Time 2) and a class that demonstrated stability (i.e., stayed in low or in high factor category across both timepoints). Overall, gender was not found to be a significant covariate, but age was significant, with younger youth demonstrating more problems at baseline than older youth. Girls were included in the analyses with the boys - perhaps partitioning out the results by gender would yield different results. Further, this study used a small sample of youthful offenders only assessed at two timepoints, and strengths were not included in the 
typologies. Without the inclusion of these variables, the taxonomic structure of offenders may be incomplete and inaccurate. Integrating both risk and strength factors can improve the completeness of type description, as well as aid in the explanatory power of these taxonomies. As such, more research is needed assessing both gender-neutral and genderresponsive risk factors and how they may help define various offender typologies.

Feminist-driven typology research. Feminist-driven typology research emerged with Daly's (1992) study, which illustrated that not all females follow a genderresponsive course into the criminal justice system. Rather, there are numerous pathways that both males and females take; some appear more gender-neutral, whereas others appear more gender-specific. Upon qualitatively examining the pre-sentence reports of 40 justice-involved women, Daly (1992) determined that there are five pathways women follow to the justice system that she labelled street women, harmed and harming women, drug-connected women, battered women, and other/economically motivated women (see Table 29 for a description of each). In an extension of her 1992 study, Daly's (1994) study tested these pathways with a sample of 40 justice-involved men from the same court, matched on age, ethnicity, and offence type. Interestingly, three of the pathways overlapped with the women, including the harmed and harming, drug-connected, and street typologies. However, one additional pathway emerged, termed the costs and excesses of masculinities pathway (see Table 29). 
Table 29

Summary of Daly's (1992 \& 1994) Pathways into Crime

\begin{tabular}{|c|c|c|}
\hline Gender & Pathway & Description \\
\hline Female & Battered Women $^{1}$ & $\begin{array}{l}\text { In an abusive relationship with romantic partner; } \\
\text { Involvement with crime is related directly to } \\
\text { negative relationship }\end{array}$ \\
\hline Female & $\begin{array}{l}\text { Other/economically } \\
\text { motivated }^{2}\end{array}$ & $\begin{array}{l}\text { Criminal involvement is directly related to having a } \\
\text { comfortable and secure lifestyle }\end{array}$ \\
\hline Both & Street category ${ }^{3}$ & $\begin{array}{l}\text { Abuse in the home leads to running away and living } \\
\text { on the streets; can lead to prostitution, drug and } \\
\text { alcohol abuse, dropping out of school, no interest in } \\
\text { employment, pro-criminal partners and continued } \\
\text { criminal activity. Described as a revolving door } \\
\text { between street living and incarceration }\end{array}$ \\
\hline Both & $\begin{array}{l}\text { Harmed and harming } \\
\text { category }\end{array}$ & $\begin{array}{l}\text { Experiences of abuse and neglect in childhood; } \\
\text { came from a chaotic home life; drug and alcohol } \\
\text { use in adolescence; emotional and psychological } \\
\text { harm with the inability to cope. }\end{array}$ \\
\hline Both & $\begin{array}{l}\text { Drug-connected } \\
\text { category }\end{array}$ & $\begin{array}{l}\text { Used and sold drugs with family members and } \\
\text { partners, limited criminal history. Not addicted to } \\
\text { drugs but use is experimental }\end{array}$ \\
\hline Male & $\begin{array}{l}\text { Costs and excesses } \\
\text { of masculinity }^{6}\end{array}$ & $\begin{array}{l}\text { Use of violence in an attempt to control others; } \\
\text { commit crimes as a way to increase social status; } \\
\text { often at the wrong place at the wrong time }\end{array}$ \\
\hline
\end{tabular}

Note. ${ }^{1}$ Battered women $(n=5,12.5 \%) ;{ }^{2}$ Other/economically motivated women $(n=4$, 10\%); ${ }^{3}$ Street category (females: $n=10,25 \%$; males: $n=16,40 \%$ ); ${ }^{4}$ Harmed and harming category (females: $n=15,37.5 \%$; males: $n=8,20 \%$ ); ${ }^{5}$ Drug-connected category (females: $n=5,12.5 \%$; males: $n=2,5 \%)$; ${ }^{6}$ Costs and excesses of masculinity ( $n$ $=14,35 \%)$.

When taken together, similarities and differences are evident. In particular, only $35 \%$ of men were part of the costs and excesses of masculinity pathway, the only uniquely male pathway. Approximately $22.5 \%$ of women made up one of the two uniquely female pathways: battered women and other/economically motivated women. 
The three pathways that were not gender-specific made up the majority of both men and women. More men than women fit into the street category ( $40 \%$ versus $25 \%$ ), whereas more women than men fit into the harmed and harming (37.5\% versus $20 \%)$ and drugconnected $(12.5 \%$ versus $5 \%)$ categories.

Since Daly $(1992,1994)$, other qualitative studies have reported similar findings, highlighting women's paths into the criminal justice system (Chesney-Lind, 1997; Dehart, 2008; Gilfus, 1992; Marquart, Brewer, Simon, \& Morse, 2001; Richie, 1996), For instance, Simpson, Yahner, and Dugan (2008) found support for gendered pathways into crime. Simpson and colleagues interviewed 351 high-risk incarcerated women and found support for Daly's drug-connected women, battered women, and harmed and harming women groupings. Unfortunately, the majority of these studies have failed to include a male comparison group and have small sample sizes (with the exception of Simpson and colleagues), which are not well suited to inferential statistics.

Recently studies using various quantitative statistical techniques have emerged, many to formulate justice-involved female typologies (e.g., Brennan, 2008; Brennan et al., 2008; Brennan, Breitenbach, Dieterich, Salisbury, \& Van Voorhis, 2012; Brown, Wanamaker, Greiner, Scott, \& Skilling, 2020; Brushett, 2013; Charak, Ford, Modrowski, \& Kerig, 2019; Jones et al., 2014; Perkins, 2010; Salisbury \& Van Voorhis, 2009). However, Brennan (2008), Brennan et al. (2008), Brown et al. (2020), and Jones et al. (2014) used samples of youth, rather than adults. Additionally, these are the only four studies, with the exception of Perkins (2010), to include a male comparison group. As such, studies examining the typologies of justice-involved women and men that incorporate gender-responsive risk factors are warranted. Nonetheless, the research that 
has been conducted on justice-involved women has provided support for Daly's (1992) gender-responsive groupings. This evidence is summarized below.

One of the first quantitative studies to test women's pathways to crime was a study conducted by Salisbury and Van Voorhis (2009). Using a sample of 313 women probationers from Missouri, USA, a path analytic approach outlined three gendered pathways to offending. The first highlights childhood victimization and the substance abuse and mental illness that follows. The second pathway highlights women's dysfunctional intimate relationships often accompanied by adult victimization, low selfefficacy, mental illness, and substance abuse. Finally, the third pathway highlights challenges in education, financial difficulties, family support, and low self-efficacy. This study, however, only included variables thought to be specific to women, failing to include gender-neutral risk factors that have garnered strong empirical support (i.e., Central Eight).

A more recent study conducted by Dehart (2018) looked at a sample of 60 women incarcerated in a maximum-security correctional facility to identify groupings first using qualitative approaches, followed by quantitative analyses to provide details on these groups. The groups were categorized by variables relevant to women's backgrounds and programming needs including: experiences of prior victimization (such as intimate partner violence), mental health concerns, and alcohol and drug use as a means of coping with violent intimate partner relationships, as well as types of offending behaviour. Five unique groupings emerged, including: 1) aggressive career offenders, which was the most common group, 2) retaliatory/ defensive violence group who offend against family, 3) child maltreatment group who offend against their own children, 4) substance dependent 
offenders experiencing intimate partner violence, and 5) social capital offenders (see

Table 30 for a further description of the five groups). While this research sheds some light on the diversity of justice-involved women, it did not incorporate justice-involved men and the sample size was small.

Table 30

Summary of Dehart's (2018) Typologies of Justice-Involved Women

\begin{tabular}{llc}
\hline Typology & Description ${ }^{1}$ & $N$ \\
\hline Aggressive career & $\begin{array}{l}\text { Generalized violence and multi-crime } \\
\text { backgrounds; substance use; experiences of } \\
\text { intimate partner violence; mental health problem }\end{array}$ & 27 \\
$\begin{array}{l}\text { Retaliatory/ } \\
\text { defensive violent - } \\
\text { family only }\end{array}$ & $\begin{array}{l}\text { Experienced severe intimate partner violence } \\
\text { (sometimes with sexual violence and stalking); } \\
\text { violent crimes in retaliation or defense of } \\
\text { themselves or loved ones }\end{array}$ & 15 \\
$\begin{array}{l}\text { Child } \\
\text { maltreatment - } \\
\text { family only }\end{array}$ & $\begin{array}{l}\text { Committing child abuse or neglect; petty criminal } \\
\text { history; substance use }\end{array}$ & 8 \\
$\begin{array}{l}\text { Substance } \\
\text { dependent }\end{array}$ & $\begin{array}{l}\text { Nonviolent offending related largely to substance } \\
\text { dependence; experienced intimate partner violence } \\
\text { (drug use linked to violent relationships); multi- } \\
\text { crime careers }\end{array}$ & 6 \\
Social capital & $\begin{array}{l}\text { Grew up in poor households; no intimate partner } \\
\text { violence and limited victimization history; limited } \\
\text { criminal history }\end{array}$ & 4 \\
\hline
\end{tabular}

Notes. ${ }^{1}$ See Dehart (2018) for a complete description of each typology.

Brennan and colleagues (2012) and Brushett (2013), in contrast, included both gender-neutral and gender-responsive variables. For instance, Brushett (2013) used a sample of 400 incarcerated women from Ohio, USA, and conducted a latent class 
analysis (LCA). Overall, three classes emerged: 1) the first class of women had high scores on antisocial attitudes and self-efficacy (a strength), 2) the second class had high scores on the criminal history, employment/financial, antisocial friends, hostility and anger, depression and anxiety, family conflict, abuse, and parental stress, and finally, 3) the last class of women had high scores on criminal history, employment/financial, antisocial peers, anger and hostility, depression and anxiety, family conflict, abuse, and parental stress. Notably, the pathways tend to include both gender-neutral and genderspecific risk/need factors. Further, this study included a few strengths (e.g., self-efficacy) which was one of the only studies to integrate risks and strengths in offending typologies. Unfortunately, this study, as well as the studies by Salisbury and Van Voorhis (2009) and Brennan et al. (2012) did not include a comparison group of men.

One study that has addressed the limitation of having no male comparison group is Perkins (2010). This study looked at 733 women and 726 men who were incarcerated in a Canadian federal penitentiary. Combining both gender-neutral and gender-responsive risk factors, this study used LCA and found that four classes emerged for the justiceinvolved women and two classes emerged for the justice-involved men. Women were classified as: 1) the potential economic and other class; 2) the problematic coping, substances, and associates class; 3) the poor mental health and coping class; and, 4) the overall high need class (each described in Table 31). In contrast, men were divided into two classes: 1) the potential economic and other class, and, 2) the problematic coping, substances, and associates class, which were parallel to the first two classes of women. 
Table 31

Summary of Perkins' (2010) Classes of Justice-Involved Men and Women

\begin{tabular}{lll}
\hline Gender & Class type $^{1}$ & Description \\
\hline Both & $\begin{array}{l}\text { Potential economic } \\
\text { and other }\end{array}$ & $\begin{array}{l}\text { Stable accommodation, educated, good coping } \\
\text { skills, positive relationships with parents, and no } \\
\text { history of mental health concerns }\end{array}$ \\
Both & $\begin{array}{l}\text { Problematic coping, } \\
\text { substances, and } \\
\text { associates }\end{array}$ & $\begin{array}{l}\text { Substance abuse and spends time with substance } \\
\text { abusing peers. Demonstrates poor coping with } \\
\text { stressful situations. }\end{array}$ \\
& $\begin{array}{l}\text { Poor mental health } \\
\text { and coping }\end{array}$ & $\begin{array}{l}\text { Unstable accommodations, poor coping with stress } \\
\text { and mental health concerns. }\end{array}$ \\
Women & Overall high need ${ }^{3}$ & $\begin{array}{l}\text { Negative parent relationships, poor coping with } \\
\text { stress, substance abuse, and associating with } \\
\text { substance abusers }\end{array}$ \\
& &
\end{tabular}

Like Daly's earlier research, this study also highlights how certain pathways to offending fit for both men and women, whereas other pathways to offending are unique for one gender over the other. This was the only study to use an adult sample to compare typologies of both men and women using a variety of risk/need, and strength variables. Unfortunately, this study was cross-sectional, and as such, further research is needed to examine if these typologies are dynamic (i.e., are these typologies stable or do they change over time?). To further advance theoretical integration and inform treatment and case management planning efforts, more research using longitudinal, multi-wave designs are required to assess offender change and/or stability over time. 
Greiner (2015) is the only known study to have conducted a multi-wave longitudinal typology study that included both traditional gender-neutral risk factors as well as a subset of hypothesized, gender-responsive need factors (e.g., internalizing mental health deficits, abuse). Using a sample of 1,354 serious adolescent offenders (184 girls and 1,170 boys) from Philadelphia and Phoenix, USA, LCA and LTA were used to examine the typologies of boys and girls that emerge as well as the stability of these typologies over time. Using four timepoints (Baseline, 12-month follow-up, 24-month follow-up, and 36-month follow-up), results indicate that youth can be classified into three typologies: A minimal-needs class_-with few needs across all domains, a comprehensive-needs class — with high needs across all domains except internalizing mental health deficits, and a complex-comprehensive-needs class-scoring high on needs across all domains. Over time, the profiles remained stable; however, at the 36-month follow-up an additional class emerged (minimal needs—substance using class) — characterized by elevated alcohol use and moderate antisocial personality, suggesting that offender profiles increase in heterogeneity over time.

Generally, more girls belonged to the minimal needs class (58\% girls vs. 55\% boys) and the complex-comprehensive needs class than boys (12\% girls vs. $5 \%$ boys), whereas more boys belonged to the comprehensive needs class than girls ( $40 \%$ boys vs. $30 \%$ girls). There were no significant gender differences in the proportions of girls and boys assigned to the three classes at Time 2 (12-month) and Time 3 (24-months followup). However, at Time 4 (36-month follow-up), there were significant gender differences in proportions of boys and girls assigned to the various classes. Specifically, there was a larger proportion of girls $(6 \%)$ than boys $(3 \%)$ in the complex-comprehensive needs 
class, as well as a larger proportion of girls (32\%) than boys $(24 \%)$ in the minimal-needs substance using class. There were the same number of boys and girls $(56 \%$ for both genders) in the minimal-needs class, however, for the comprehensive-needs class; there were a higher proportion of boys (18\%) than girls (7\%). Overall, while this study was the first to examine the clustering of gender-neutral and gender-responsive risk factors among a mixed-gender sample using a multi-wave, longitudinal design, this research was conducted on a sample of youth — thus, whether these results are generalizable to justiceinvolved adults is unknown.

\section{Summary and Study 2 Rationale}

Collectively, research indicates that there are more gender-similarities, rather than gender-differences in offender typologies. This body of research, however, has typically relied on relatively small samples of justice-involved females and has refrained from examining strength factors in typology construction. As such, more research is needed on the risk and strength typologies of justice-involved adults, and how they may change over time.

The current study examines theoretically-integrated typologies that merge tenets of both gender-neutral, gender-responsive, and strengths-based paradigms. This research is conducted to help inform the treatment and rehabilitation of justice-involved men and women. Specifically, if there is evidence to suggest that certain risk and strength factors cluster together among subsets of men and women, treatment programs, and supervision practices could be tailored in accordance. The current study assesses whether there are changes in typological membership over time, providing further understanding of how dynamic risk and strength factors may change over time. 
Research Question 1: Can meaningful offender typologies be delineated among justice-involved men and women using variables informed by mainstream, feminist pathways, and strengths-based perspectives? In line with previous research examining criminal typologies of offenders (e.g., Greiner, 2015; Perkins, 2010), it is hypothesized that several unique groups will emerge. Because this is the first study to include strengths in the development of typologies, this research is exploratory in nature. Importantly, as previously mentioned, the number of typologies that emerge depend on the type of analysis and the number of variables being included in the analysis. It is expected that between four and six typologies will emerge, for both men and women, which is consistent with previous typology-building studies with justice-involved adolescents and adults (e.g., Dehart, 2018; Perkins, 2010).

Research Question 2: Are these offender typologies that emerged in research question 1 stable over time? Because most typological research has been cross-sectional in nature (e.g., Salisbury \& Van Voorhis, 2009) and given the fact that the only longitudinal gender-responsive typological research was conducted on adolescent samples (e.g., Greiner, 2015), the assessment of whether these typologies are stable or change over time among adults will be exploratory in nature. As such, no hypotheses are made with respect to whether the typologies will remain stable or not.

Research Question 3: Does typology membership differ based on gender? Both qualitative and quantitative feminist studies have found that women have unique histories of abuse, mental health issues, and substance abuse (Belknap, 2015). However, genderneutral research has found support that women and men share similar risk factors (e.g., Andrews et al., 2012; Green \& Campbell, 2006). Further, previous typological research 
has found support for risk and need clusters that are not mutually exclusive for boys and men and girls and women (e.g., Daly, 1994; Jones et al., 2014). As such, it is hypothesized that there will be similar typologies that emerge for both men and women. It is also expected that at least one typology will emerge comprised predominately of gender-responsive need and strength factors (e.g., abuse and family issues, mental health issues) for both men and women.

Research Question 4: Is typology (profile) membership stable over time? That is, will the proportions of individuals belonging to each typology remain stable across timepoints or will proportions change as time progresses (i.e., will individuals switch profiles as time progresses)? It is important to note that this research question will only be answered if the typologies remain stable over time. Because this is the first study to examine longitudinal typologies among adults incorporating risks and strengths, no hypotheses are made.

Research Question 5: Is typology membership related to reoffending outcomes? The association between typological structure and criminal outcomes (i.e., technical violations, any new charges, violent charges, and any negative outcome) is important for guiding offender treatment. Because longitudinal gender-responsive typological research has been conducted on adolescent samples (e.g., Greiner, 2015), no hypotheses are made with respect to the association among various typologies and reoffending outcomes among men and women. 


\section{Method for Study 2}

\section{Participants and Research Design}

Similar to Study 1, this study used archival SPIn assessment data from Orbis Partners (see Participant section in Study 1 for a more detailed description of the larger original SPIn database). The current study consisted of men and women who initially started community supervision in Alberta, Canada between 2009 and 2012 (Mdn year = 2012). Each individual was serving a provincial sentence for an index offence(s) with some sentences immediately starting under community supervision (conditional sentence order or probation order) and others starting community supervision post-release from a provincial correctional facility.

Current study. The current study consisted of men and women who were assessed across three timepoints and were successful over a 9 to 14 -month period (depending on time of the third assessment). That is, men and women with at least three assessments were utilized, as long as one assessment came from Time $1=$ the initial SPIn Full Assessment that was assessed within 90 days of start of supervision, Time $2=3$ to 8 months post initial SPIn assessment, and Time $3=9$ to 14 months post initial SPIn assessment. If an individual had multiple assessments during one time period, a random assessment was selected to represent that point in time. These specific time periods allowed for the largest sample inclusion and adhered to SPIn re-assessment guidelines, which state that SPIn re-assessments should occur every six months. Individuals who recidivated prior to having a completed assessment at each timepoint selected above were removed from analyses (to assess transitions among profiles, data is required on all timepoints for all cases). This resulted in a sample of 1,684 women and 6,675 men (see Figure 12). 


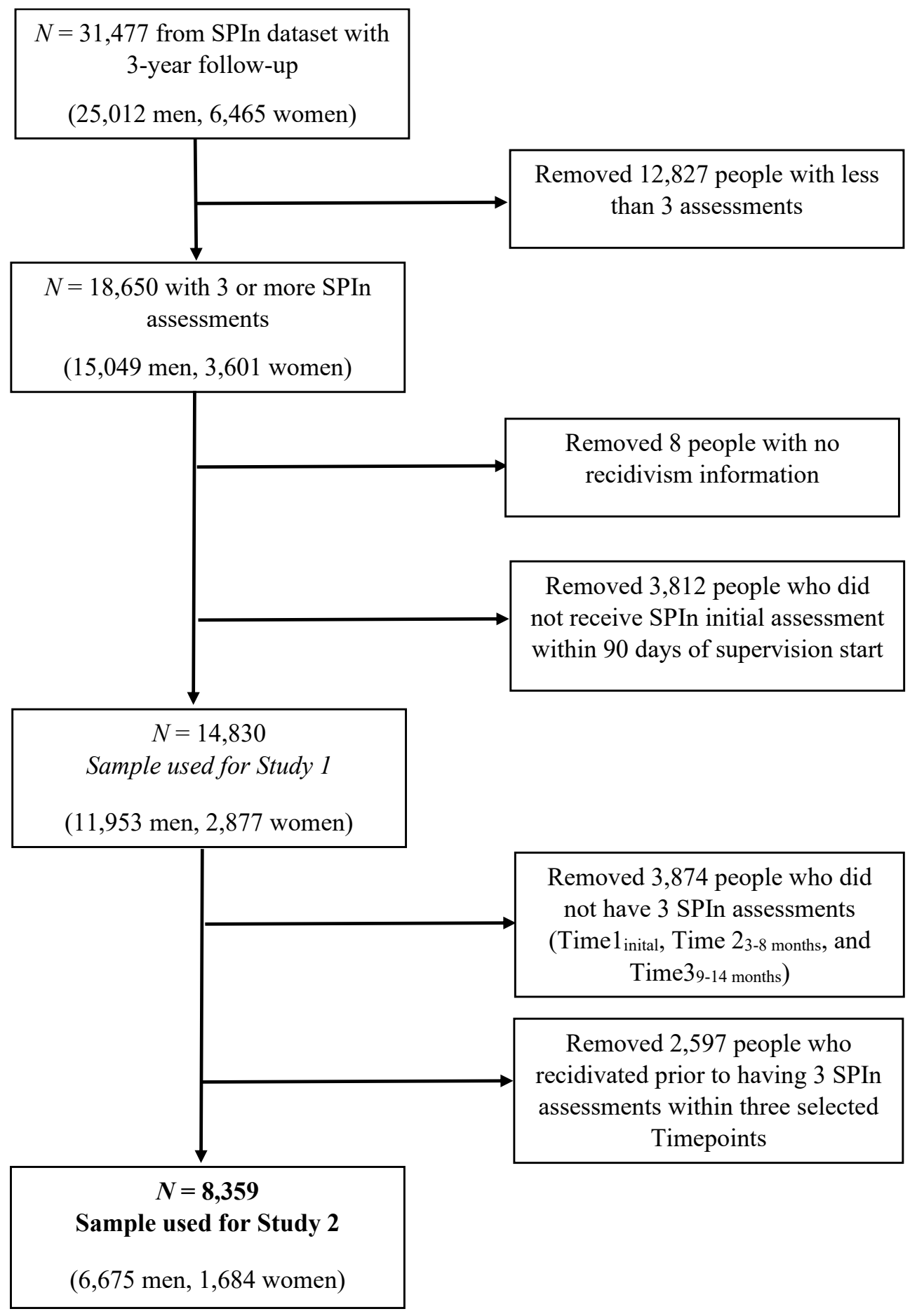

Figure 12. Participant flow chart for Study 2. 
Descriptives. Based on SPIn Full Assessment static risk scores, the majority of the men and women were low risk $(\operatorname{men}=55.1 \%$; women $=62.5 \%)$, about a third were moderate risk $($ men $=38.6 \%$; women $=33.3 \%$, with very few who scored high risk $($ men $=6.4 \%$; women $=4.3 \%$ ). As Table 32 illustrates, men were slightly older than women (34.4 vs. 33.3 years old). A higher proportion of women were Indigenous (23.9\%) compared to men $(13.0 \%)$, and more women had at least one mental health flag than the men $(64.2 \%$ vs. $43.0 \%)$

In terms of index offences, a higher proportion of women committed a nonviolent offence (36.8\% vs. $23.4 \%$ ), whereas a higher proportion of men committed a violent offence $(42.8 \%$ vs. $30.5 \%)$ and a sexual offence (4.6\% vs. $1.1 \%)$. For the SPIn initial Full Assessment, there were no significant gender differences on total dynamic risk score, however, men scored higher than women on both the total static risk score $(22.3$ vs. 18.5$)$ and the total dynamic strength score (28.7 vs. 27.7). Finally, in terms of recidivism, there was a significant difference between men and women for new violent charges and for any new charges, whereby more men (6.2\% and $10.7 \%$, respectively) than women $(3.6 \%$ and $8.8 \%$, respectively) were charged with at least one new violent offence and one new offence within 3-years after the initial SPIn Full Assessment. 
Table 32

Descriptive Information for Men and Women and Gender Differences

\begin{tabular}{|c|c|c|c|c|c|c|c|}
\hline \multirow[b]{2}{*}{ Variable } & \multicolumn{2}{|c|}{$\begin{array}{c}\text { Men } \\
(N=6,675)\end{array}$} & \multicolumn{2}{|c|}{$\begin{array}{c}\text { Women } \\
(N=1,684)\end{array}$} & \multirow[b]{2}{*}{$t$} & \multirow[b]{2}{*}{$\gamma^{2}$} & \multirow[b]{2}{*}{$d$} \\
\hline & $M(S D)$ & $\%(n)$ & $M(S D)$ & $\%(n)$ & & & \\
\hline \multicolumn{8}{|l|}{ Demographics } \\
\hline Age & $34.4(11.6)$ & & $33.3(11.0)$ & & $3.33 * *$ & & .09 \\
\hline Indigenous & & $13.0(867)$ & & $23.9(403)$ & & $124.96^{* *}$ & .24 \\
\hline Any custody & & $9.9(663)$ & & $8.2(138)$ & & $4.69^{*}$ & .05 \\
\hline MH flag ${ }^{1}$ & & $43.0(2,873)$ & & $64.2(1,081)$ & & $252.55^{* *}$ & .35 \\
\hline \multicolumn{8}{|l|}{ Index Offence ${ }^{2}$} \\
\hline Sexual & & $4.6(310)$ & & $1.1(18)$ & & $45.60 * *$ & .15 \\
\hline Violent & & $42.8(2,856)$ & & $30.5(513)$ & & $84.88 * *$ & .20 \\
\hline Non-violent & & $23.4(1,559)$ & & $36.8(619)$ & & $125.36^{* *}$ & .24 \\
\hline Admin. & & $4.3(286)$ & & $5.2(87)$ & & 2.45 & .03 \\
\hline Other & & $13.0(869)$ & & $12.9(218)$ & & 0.01 & .00 \\
\hline \multicolumn{8}{|l|}{ Full Assessment info ${ }^{3}$} \\
\hline Static risk & $22.3(16.9)$ & & $18.5(14.9)$ & & $8.37 * *$ & & .23 \\
\hline Dynamic risk & $17.5(13.9)$ & & $17.1(14.8)$ & & 1.07 & & .03 \\
\hline Dynamic strength & $28.7(16.7)$ & & $27.7(17.2)$ & & $2.30 *$ & & .06 \\
\hline \multicolumn{8}{|l|}{ Recidivism $^{4}$} \\
\hline Any negative ${ }^{5}$ & & $13.0(868)$ & & $11.5(193)$ & & 2.89 & .04 \\
\hline Technical & & $6.3(420)$ & & $5.8(97)$ & & 0.66 & .02 \\
\hline New violent & & $6.2(412)$ & & $3.6(61)$ & & $16.38 * *$ & .09 \\
\hline Any new & & $10.7(717)$ & & $8.8(148)$ & & $5.53 *$ & .05 \\
\hline
\end{tabular}

Note. $M=$ Mean. $S D=$ Standard deviation. $t=$ t-test. $\chi^{2}=$ Chi square. $d=$ Cohen's d effect size. ${ }^{1} \mathrm{MH}=$ Mental Health. ${ }^{2}$ Non-violent $=$ property,

theft, fraud, drug offences, impaired driving. Admin $=$ technical violations and administration of justice offences. Other $=$ traffic and other offences. ${ }^{3}$ Assessment info $=$ based on initial SPIn Full Assessment. ${ }^{4}$ Recidivism $=$ based on 3-year follow-up information. ${ }^{5}$ Any negative outcome $=$

includes any technical violations or new charges. Missing data on the index offence was $11.9 \%$ for men and $13.6 \%$ for women. ${ }^{*} p<.05,{ }^{* *} p<.001$ 


\section{Measures}

The Service Planning Instrument (SPIn) - Full Assessment. The SPIn (Orbis Partners, 2003) is a risk, need and strength assessment and case management planning instrument used with adults in both institutional and community-based justice settings. Information obtained from semi-structured interviews and file-reviews are used to score the Pre-Screen version and/or the Full Assessment version of the SPIn. The full SPIn assessment contains 90 items, of which 35 are used to calculate the Pre-Screen risk and strength scores. For the purpose of the current study, however, focus will be placed on the SPIn Full Assessment. The 90 items from the Full Assessment, make up 11 domains, which include: Criminal history, response to supervision (e.g., institutional misconducts, violations), aggression, substance use, social influences (e.g., negative and prosocial peers), family, employment and education, attitudes, social and cognitive skills, stability (e.g., living situation, financial situation), and mental health. For a detailed description of the SPIn, see Study 1 and Appendix A.

Importantly, specific measures for the current study are described as a function of their role in the analysis. Indicator variables are gender-responsive, gender-neutral, or strength variables that are deemed endogenous to latent profiles - that is, they are utilized for typology formation (Vermunt \& Magidson, 2002). The indicator variables for the current analyses all stemmed from domain scores from the SPIn Full Assessment. Covariates, in contrast, are considered exogenous to the model — used to predict profile membership and improve classification accuracy (Vermunt \& Magidson, 2002). Finally, auxiliary variables, not used directly in the analysis model, are examined after typological 
classifications to test the equality of proportions (Muthén \& Muthén, 1998-2017).

Descriptions of each variable type and measures used are followed.

SPIn-derived LPA/LTA indicators. The following 18 SPIn-derived variables were included as indicator variables to form latent profiles in the analyses. More details, such as sample item descriptions and scoring, can be found in Table 1A and Table 2A in Appendix A.

Criminal history—Static risk domain. The criminal history static risk domain assesses factors related to the individuals past offences, including youth dispositions, previous adult convictions, age at first arrest, and past incarcerations. Scores on this domain are based on six items and range from 0 to 20, with scores from 1 to 3 indicating low risk, scores of 4 to 8 indicating moderate risk, and scores of 10 or more indicating high risk. Internal consistency was adequate for both men $(\alpha=.76)$ and women $(\alpha=.72)$. Aggression/violence_Dynamic risk domain. The aggression and violence dynamic risk domain assesses a host of factors related to violent convictions, behaviour, and beliefs that put an individual at higher risk for reoffending, including opinions on verbal and physical aggression and frequency of conflicts. Total scores on this domain are based on four items and range from 0 to 8 , with scores of 0 to 1 indicating none to low risk, scores of 2 to 3 indicating moderate risk, and scores of 4 or more indicating high risk. Internal consistency was good for both men $(\alpha=.86)$ and women $(\alpha=.80)$. Aggression/violence_Dynamic strength domain. The aggression and violence dynamic strength domain assesses opinions and beliefs about threatening behaviour that would decrease an individual's risk for reoffending. Total scores on this domain are based on four items and ranges from 0 to 8 , with scores of 1 to 2 indicating a low strength 
score on aggression, a score of 3 to 4 indicating a moderate strength score on aggression, and a score of 5 or more indicating high strength. Internal consistency was very good for both men $(\alpha=.88)$ and women $(\alpha=.89)$.

Substance use-Dynamic risk domain. The substance use dynamic risk domain assesses the types and number of times using various drugs and alcohol and whether it disrupts functioning. This domain assesses the use of eleven different substances including: alcohol, marijuana, cocaine/crack, ecstasy or other club drugs, heroin, hallucinogens, inhalants, amphetamines, methamphetamines, prescription drug misuse, and any other substances causing disruptions in that person's life. Total scores range from 0-28, where scores from 1 to 4 indicate low risk, scores of 5 to 17 indicate moderate risk, and scores of 18 or more indicate high risk. This scale is made up of three main items, with sub-items for each type of substance, making it difficult to assess internal consistency, as evidenced by low internal consistency findings for men $(\alpha=.47)$ and women $(\alpha=.39)$.

Social Influences—Dynamic risk domain. The social influences dynamic risk domain assesses antisocial peers and community engagement, associations with gangs, and negative influences that may put an individual at risk for future criminal behaviour. This domain is scored based on six items and total scores range from 0 to 26 , with scores of 1 and 2 indicating low risk, scores of 3 to 6 indicating moderate risk, and scores of 7 or more indicating high risk. Internal consistency for this domain was adequate for both men $(\alpha=.61)$ and women $(\alpha=.64)$.

Social Influences-Dynamic strength domain. The social influences dynamic strength domain assesses positive social activity and community engagement, as well as 
prosocial peer relationships that may act as a support. Overall, this domain is based on five items, and total scores range from 0 to 15 , with scores of 1 to 4 indicating low strength, scores of 5 to 8 indicating moderate strength, and scores of 9 or more indicating high strength. This domain was not found to have good internal consistency for either men $(\alpha=.56)$ or women $(\alpha=.56)$.

Family_Dynamic risk domain. The family dynamic risk domain assesses an individual's negative family and intimate relationships, as well as marital factors, and parental factors that may increase risk of criminal behaviour. This domain is scored across seven items with total scores ranging from 0 to 26 . Scores of 1 and 2 indicate low risk, scores of 3 to 7 indicate moderate risk, and scores of 8 or more indicate high risk. Internal consistency was poor for both men $(\alpha=.46)$ and women $(\alpha=.49)$.

Family-Dynamic strength domain. The family dynamic strength domain assesses positive family and marital relationships in an individual's life, as well as pro-social models, attachment to children and family involvement. This domain is made up of seven items with total scores ranging from 0 to 14 . Scores of 1 to 3 indicate low strength, scores of 4 and 5 indicate moderate strength, and scores of 6 or more indicate high strength. Internal consistency was adequate for both men $(\alpha=.65)$ and women $(\alpha=.62)$.

Employment-Dynamic risk domain. The employment dynamic risk domain assesses employment performance and plans, as well as job search skills. This domain is made up of six items and total scores range from 0 to 14 . Scores of 1 and 2 indicate low risk, scores of 3 to 6 indicate moderate risk, and scores of 7 or more indicate high risk. Internal consistency was found to be good for men $(\alpha=.76)$ and for women $(\alpha=.75)$. 
Employment-Dynamic strength domain. The employment dynamic strength domain assesses an individual's marketability, education, and job search skills which may assist in reducing one's likelihood of reoffending. This domain is made up of five items with total scores ranging from 0 to 12 . Scores of 1 and 2 indicate low strength, scores of 3 to 7 indicate moderate strength, and scores of 8 or more indicate high strength. The internal consistency was good for men $(\alpha=.76)$ and for women $(\alpha=.75)$. Attitudes-Dynamic risk domain. The attitudes dynamic risk domain assesses attitudes towards crime and commitment to criminal lifestyle, and attitude towards the criminal justice system. This domain is made up of nine items and total scores range from 0 to 14 . Scores of 1 and 2 indicate low risk, scores of 3 to 5 indicate moderate risk, and scores of 6 or more indicate high risk. Internal consistency was good for men $(\alpha=.82)$ and for women $(\alpha=.77)$.

Attitudes-Dynamic strength domain. The attitudes dynamic strength domain assesses law-abiding attitudes, ability to accept responsibility, will to make amends, and change readiness. This domain is made up of nine items and total scores range from 0 to 14. Scores of 1 to 5 indicate low strength, scores of 6 to 10 indicate moderate strength, and scores of 11 or more indicate high strength. Internal consistency was very good for men $(\alpha=.87)$ and for women $(\alpha=.87)$.

Social/cognitive skills_Dynamic risk domain. The social and cognitive skills dynamic risk domain assess hostility, impulsivity, and negative problem solving that may be related to future offending behaviour. This domain is made up of eight items and total scores range from 0 to 18 . Scores of 1 and 2 indicate low risk, scores of 3 and 4 indicate 
moderate risk, and scores of 5 or more indicate high risk. Internal consistency was very good for men $(\alpha=.86)$ and for women $(\alpha=.83)$.

Social/cognitive skills-Dynamic strength domain. The social and cognitive dynamic strength domain assesses problem solving skills, goal setting, behavioural control, and interpersonal skills. This domain consists of eight items and total scores range from 0 to 18 . Scores from 1 to 3 indicate low strength, scores of 4 to 9 indicate moderate strength, and scores of 10 or more indicate high strength. Internal consistency is very good for both men $(\alpha=.87)$ and women $(\alpha=.87)$.

Stability—Dynamic risk domain. The stability dynamic risk domain assesses financial, accommodation, and transportation concerns. This domain is made up of four items and total scores range from 0 to 13 . Scores of 1 and 2 indicate low risk, scores of 3 to 5 indicate moderate risk, and scores of 6 or more indicate high risk. Internal consistency is found to be poor for both men $(\alpha=.51)$ and women $(\alpha=.49)$, which may be due to the small number of items making up this domain.

Stability-Dynamic strength domain. The stability dynamic strength domain assesses an individual's life skills, financial situation and accommodations. This domain is made up of four items and ranges in scores from 0 to 7 . Scores of 1 to 3 indicate low strength, a score of 4 indicates moderate strength, and a score of 5 or more indicates a high strength. Internal consistency was poor for men $(\alpha=.48)$ and for women $(\alpha=.42)$, which may be due to the small number of items making up this domain.

Mental health flag. The mental health flag is a count variable of mental health concerns, aggregated into a variable rated from 0 (no flags) to 2 (two or more flags). This variable assesses history of mental health conditions such as suicidal ideation, sexual 
aggression, victimization, and self-injurious behaviours. Due to the limited number of items, internal consistency could not be examined.

Adverse Childhood Experiences. The original ACEs (Felitti et al., 1998) was created to examine the relationship between negative childhood experiences and negative outcomes in adulthood. Specifically, the ACEs study conducted by Felitti et al. (1998) found that having a greater number of the 10 key negative childhood experiences (scored $0=$ absent; $1=$ present) increases the likelihood of problems with alcoholism, drug abuse, depression, suicide attempts, severe obesity, and diseases in adulthood. A proxy ACE score was calculated from the SPIn Full Assessment using the following items: uses substance use to cope with trauma, comes from a single parent home, experienced physical abuse, experienced sexual abuse, experienced violence in the home, experienced instability in the home or foster care, parental substance use, and parental mental health issues. For each of these eight items deemed present, a score of 1 was added, to obtain a total score ranging from 0 to 8 . Notably, the original ACEs was comprised of 10 items, however, only 8 items could be scored by proxy using the SPIn. This method has demonstrated validity (Baglivio, Wolff, Piquero, \& Epps, 2015), and the SPIn-derived ACE measure evidenced adequate reliability for men $(\alpha=.64)$ and women $(\alpha=.71)$.

Covariates. While indicators are used to inform profile formation, covariates are used to predict profile membership. By including covariates into the model, classification accuracy is improved (Lubke \& Muthén, 2007). Covariates are factors that are thought to potentially impact profile classification and therefore are controlled for when building the typologies at each timepoint. After the LPAs were ran at each timepoint, covariates were examined to determine their impact on profile formation. In the current analyses, two 
continuous covariates were included at each timepoint: Age and total static risk score from the full SPIn assessment.

Total static risk score. The total static risk score obtained from the SPIn Full Assessment was included to control for any differences that would result specifically from individuals' static factors. Static factors are unchangeable factors, such as historical information (e.g., response to supervision, history of homelessness) and thus, remain relatively stable over time. Total static risk scores ranging from 1 to 20 indicate low scores on static risk, scores ranging from 21 to 47 indicate moderate scores on static risk, and scores of 48 or more indicate high score on static risk. Internal consistency was found to be good for both women $(\alpha=.81)$ and men $(\alpha=.84)$.

Age. Age at time of initial SPIn assessment was used as the second covariate to control for any differences that would result as a product of individuals' biological age. The age ranged from 17 to 74 for women $(M=33.3, S D=11.0)$ and ranged from 16 to 83 for men $(M=34.4, S D=11.6)$ at the start of supervision.

Auxiliary variables. In contrast, auxiliary variables are not used directly in the analysis model, instead they are examined after the LPAs are conducted to see if there are differences in proportions in the composition of the typologies that emerged. Indigenous status was specified as an auxiliary variable.

Indigenous status. Indigenous status was used post-analysis, to assess the extent to which profile membership varied among those who are non-Indigenous and those who are Indigenous (i.e., First Nations, Metis, or Inuit). Indigenous status was a dichotomous variable, used to indicate if the individual self-identified as Indigenous (yes/no). 
Distal outcomes. There were four distal outcomes of interest that were examined independently: (1) technical violations, which include only incidents that violate or breach conditions while on supervision, (2) any violent charges, which includes violent charges against the person such as harassment, assault, and weapons offences, (3) any new charges, which includes charges for any new criminal offence, including any violent offence, property offence, theft, or drug-related offence, and (4) any negative outcome, which includes technical violations or any recontact with the criminal justice system. Refer to measures section in Study 1 for a detailed description of various outcomes. Each of these are dichotomous outcomes (yes/no) and were assessed over a three-year fixed follow-up period from the start of community supervision (which translates to 22 to 27 months post Time 3, as Time 3 assessments occurred between 9 and 14 months post initial assessment).

\section{Analyses}

Data cleaning was first conducted in SPSS (version 25; IBM, 2017), and the primary analysis was conducted in MPlus version 8 (Muthén \& Muthén, 1998-2017). See Appendix G, Table $1 \mathrm{G}$ for Analyses used in Study 2.

\section{Latent profile analysis (LPA) and latent transition analysis (LTA). In} recognition that criminal behaviour and risk factors for criminal behaviour are not stagnant and vary from individual to individual, researchers have begun to incorporate person-centered approaches to modeling crime trajectories (Piquero, Reingle Gonzalez, \& Jennings, 2015). Instead of focusing on the relationships among variables, these models focus specifically on the behaviour of individuals. That is, LPA, unlike factor analysis, classifies individuals into various typologies based on comparable patterns of 
individual characteristics (Collins \& Lanza, 2010; Piquero et al., 2015). In this case, men and women are clustered based on parallel patterns of responses on various indicators (McCutcheon, 1987), informed from both gender-neutral, gender-responsive, and strengths-based research.

While LPA is used for cross-sectional data with one assessment timepoint, LTA is used for multi-wave studies with multiple timepoints. As such, LTA measures typology stability or change over time (Collins \& Lanza, 2010). Specifically, LTA is beneficial for examining changes in latent profiles with longitudinal data because it assesses transitional probabilities, which informs the probability of transitioning between profiles at different times (Collins \& Lanza, 2010).

To determine the relative model fit across the various numbers of profiles, several fit indices criteria were used. The fit indices examined included the Akaike's Information Criterion (AIC), the Bayesian Information Criterion (BIC), and the sample-size adjusted Bayesian Information Criterion (ABIC); lower values on each of these indices indicated better model fit. Entropy was also examined and values closer to 1 indicated better model fit. Entropy is a measure of how well profiles can be distinguished based on each individual's estimated profile probability. Lo-Mendell-Rubin test was also used to determine whether a $k$ profile model fit the data better than a $k$-1 profile model. Overall, the best fitting model was determined based on fit indices criteria, theory, and through examination and interpretation of the various profile structures.

Steps used to examine LPA and LTA. The current analyses examined: (1) gender-informed treatment typologies that incorporate both gender-neutral and genderresponsive strengths and risk/need factors, (2) how these typologies change or remain 
stable over time, and (3) how these typologies are related to reoffending. As such, there were 6 steps used to complete these analyses (see Appendix G for list of steps). In step 1, a latent profile analysis (LPA) was conducted for men and women independently, to examine whether a meaningful latent profile emerged. Fit indices were used to determine the number of profiles that best fit the data. In step 2, if meaningful profiles emerged at Time 1, LPAs for Time 2 and Time 3 were conducted (part of latent transition analysis [LTA]) to examine the number of profiles that emerged at these later timepoints. In step 3, the two covariates, age and total static risk score, were examined at each timepoint and for men and women independently, to determine their impact on profile formation. In step 4, the auxiliary variable, Indigenous status, was examined at each timepoint for men and women, to determine the proportions of each profile that were made up of Indigenous Peoples. In step 5, latent transition probabilities (the main component of LTA) were examined between each timepoint to assess changes in profile membership over time. Notably, latent transitions can only be examined if similar profiles emerged at each timepoint. Finally, in step 6, the relationship between latent transitions and four distal outcomes (technical violation, any new charges, violent charges, and any negative outcome) were examined.

\section{Results}

\section{Data Screening}

There were no missing data across all three timepoints for any of the 18 indicator variables used to generate LPA/LTA profiles. There was also no missing data on the covariates and auxiliary variables. Covariance matrices for the variables used in the LPAs were examined to assess coverage (minimum threshold for good coverage is .10; Muthén 
\& Muthén, 1998-2017). The covariance coverage for all three timepoints indicated good coverage.

Two count variables (mental health and adverse childhood experiences) had a large proportion of participants who scored zero (over $50 \%$ across each timepoint). As such, a zero-inflated Poisson regression was specified for these variables to account for the large number of zeros. ${ }^{20}$

The majority of domain scores across all timepoints were positively skewed (See Tables $1 \mathrm{H}, 2 \mathrm{H}$, and $3 \mathrm{H}$ in Appendix $\mathrm{H}$, which report the mean, standard deviation, and range for each of the variables included in the LPAs at each of the three timepoints). Given that it is expected that latent profile models are made up of a variety of normal distributions from different types of offenders, variables are treated as normally distributed continuous variables (Kreuter \& Muthén, 2008). Although the main assumption of latent variable models is local independence (which assumes that the latent variables explain why observed variables are related, and therefore all variables within each profile are unrelated), this assumption is only required to hold for models with dichotomous variables (Magidson \& Vermunt, 2004), and as such was not tested.

\section{LPA Time 1}

A 2, 3, 4, 5, and 6-profile model solution were run sequentially to identify the best fitting model for men and women, independently (see Table 33). Upon considering the model fit indices, theory, and the interpretation of the various profiles, it was

\footnotetext{
${ }^{20}$ Poisson regression account for the large proportion of zeros and provides two parameter estimates: (1) a count variable representing values from cases where we are able to assume values of zero and above, and (2) an inflation variable whereby a score of 1 indicates that that case is only able to assume a value of zero. This variable is treated as a binary latent variable (Muthén \& Muthén, 1998-2017).
} 
determined that a 3-structure profile solution fit the data best for women, whereas a 5structure profile solution for the data the best for men.

Table 33

Relative Fit Statistics for Time 1 for Men and Women, Independently

\begin{tabular}{lcccccc}
\hline \multicolumn{7}{c}{ Women } \\
Profile & AIC & BIC & ABIC & Entropy & LMR & $\boldsymbol{p}$ \\
\hline 2-Structure & 135729.14 & 136027.73 & 135852.99 & .932 & 4828.54 & $<.001$ \\
3-Structure & $\mathbf{1 3 2 2 8 6 . 4 7}$ & $\mathbf{1 3 2 6 8 8 . 2 1}$ & $\mathbf{1 3 2 4 5 3 . 1 2}$ & $\mathbf{. 9 1 9}$ & $\mathbf{3 4 5 6 . 1 8}$ & $\mathbf{. 0 0 7}$ \\
4-Structure & 130391.31 & 130896.20 & 130600.75 & .933 & 1919.56 & .081 \\
5-Structure & 129149.88 & 129757.92 & 129402.11 & .927 & 1270.43 & .160 \\
6-Structure & 128116.48 & 128827.67 & 128411.50 & .938 & 1063.87 & .224 \\
\hline & & & Men & & & \\
\hline Profile & $\mathbf{A I C}$ & $\mathbf{B I C}$ & ABIC & $\mathbf{E n t r o p y}$ & LMR & $\boldsymbol{p}$ \\
\hline 2-Structure & 541479.42 & 541853.76 & 541678.98 & .901 & 16540.76 & $<.001$ \\
3-Structure & 528938.44 & 529442.09 & 529206.94 & .920 & 12504.25 & .029 \\
4-Structure & 522340.77 & 522973.74 & 522678.21 & .940 & 6596.24 & .015 \\
5-Structure & $\mathbf{5 1 7 3 7 7 . 3 0}$ & $\mathbf{5 1 8 1 3 9 . 5 8}$ & $\mathbf{5 1 7 7 8 3 . 6 7}$ & $\mathbf{. 9 0 7}$ & $\mathbf{4 9 7 1 . 7 6}$ & $<.001$ \\
6-Structure & 514070.71 & 514962.32 & 514546.03 & .905 & 3324.71 & .146 \\
\hline
\end{tabular}

Note. $\mathrm{AIC}=$ Akaike's Information Criterion. $\mathrm{BIC}=$ Bayesian Information Criteria. ABIC $=$ Sample size adjusted Bayesian Information Criteria. LMR $=$ Lo-Mendell-Rubin test.

Posterior profile membership probabilities were also examined (see Table 34).

The probabilities of correct classification for the 3-Profile structure for women ranged from .95 to .97 , and for the 5-Profile structure for men ranged from .90 to .98 , which is considered good. 
Table 34

Posterior Profile Membership Probabilities for Time 1 for Men and Women

\begin{tabular}{cccccc}
\hline & \multicolumn{5}{c}{ Women } \\
\hline Most Likely Profile & \multicolumn{5}{c}{ Latent Profiles } \\
\cline { 2 - 6 } Membership & 1 & 2 & 3 & \\
\hline 1 & .966 & .017 & .018 & \\
2 & .025 & .967 & .007 & \\
3 & .042 & .009 &. $\mathbf{9 4 8}$ & \\
\hline Most Likely Profile & & Men & & \\
Membership & 1 & 2 & 3 & .000 \\
\hline 1 & .961 & .000 & .039 & .003 \\
2 & .000 & .982 & .014 & .001 & .005 \\
3 & .055 & .002 & .901 & .038 & .000 \\
4 & .002 & .000 & .048 & .950 & .0003 \\
5 & .001 & .003 & .032 & .000 & .963 \\
\end{tabular}

Note. Probability of Profile membership classification equal to or greater than .70 is considered acceptable.

Time 1 profile descriptions for women. For the women, the profiles are labelled as follows: Profile $1=$ Low risk/low strength $(n=822 ; 48.8 \%)$, Profile $2=$ Gender-responsive, low risk/high strength $(n=537 ; 31.9 \%)$, and Profile $3=$ Aggressive, complex need/low strength $(n=325 ; 19.3 \%)$. The estimated means and standard deviations for each of the profiles were identified and labelled for women (see Table $35)$. 
Table 35

Means for Each of the Risk and Strength Domains across the Three Profiles of Women at Time 1

\begin{tabular}{|c|c|c|c|c|c|c|}
\hline \multirow[b]{2}{*}{ Variables in LPA } & \multicolumn{2}{|c|}{$\begin{array}{l}\text { Profile 1 } \\
(n=822)\end{array}$} & \multicolumn{2}{|c|}{$\begin{array}{l}\text { Profile } 2 \\
(n=537)\end{array}$} & \multicolumn{2}{|c|}{$\begin{array}{l}\text { Profile } 3 \\
(n=325)\end{array}$} \\
\hline & $M$ & $S D$ & $M$ & $S D$ & $M$ & $S D$ \\
\hline Criminal history & 3.01 & $(3.27)$ & 4.24 & $(4.41)$ & 6.82 & $(5.23)$ \\
\hline Aggression $^{1}-$ Risk & 0.07 & $(0.37)$ & 0.10 & $(0.45)$ & 1.02 & $(1.58)$ \\
\hline Aggression - Strength & 0.70 & $(1.30)$ & 4.10 & $(1.87)$ & 1.63 & $(1.59)$ \\
\hline Substance use - Risk & 3.86 & $(5.55)$ & 5.97 & $(8.04)$ & 10.15 & $(9.24)$ \\
\hline Social influence - Risk & 1.43 & $(2.18)$ & 1.94 & $(2.20)$ & 6.06 & $(4.01)$ \\
\hline Social influence - Strength & 3.34 & $(2.27)$ & 7.47 & $(3.23)$ & 2.92 & $(2.52)$ \\
\hline Family - Risk & 3.16 & $(3.24)$ & 3.99 & $(3.63)$ & 7.63 & $(5.05)$ \\
\hline Family - Strength & 2.58 & $(2.03)$ & 6.00 & $(2.95)$ & 2.77 & $(1.92)$ \\
\hline Employment - Risk & 0.36 & $(0.89)$ & 0.55 & $(1.29)$ & 3.82 & $(3.09)$ \\
\hline Employment - Strength & 1.59 & $(1.37)$ & 4.44 & $(3.22)$ & 0.99 & $(1.49)$ \\
\hline Attitudes - Risk & 0.43 & $(0.99)$ & 0.41 & $(0.93)$ & 2.51 & $(2.61)$ \\
\hline Attitudes - Strength & 3.55 & $(2.37)$ & 11.26 & $(3.83)$ & 4.53 & $(3.30)$ \\
\hline Skills $^{2}-$ Risk & 0.24 & $(0.74)$ & 0.15 & $(0.51)$ & 2.59 & $(2.64)$ \\
\hline Skills - Strength & 1.97 & $(1.67)$ & 8.64 & $(3.49)$ & 1.60 & $(2.00)$ \\
\hline Stability - Risk & 0.96 & $(1.35)$ & 1.43 & $(1.67)$ & 4.11 & $(2.30)$ \\
\hline Stability - Strength & 3.24 & $(1.20)$ & 4.78 & $(1.38)$ & 2.45 & $(1.49)$ \\
\hline Mental health - Flag & 0.89 & $(0.90)$ & 1.20 & $(0.89)$ & 1.59 & $(0.75)$ \\
\hline $\mathrm{ACEs}^{3}$ & 0.68 & $(1.13)$ & 1.84 & $(1.79)$ & 2.82 & $(1.88)$ \\
\hline
\end{tabular}

Note. $M=$ Mean, $S D=$ Standard deviation. Profile $1=$ Low risk/low strength, Profile $2=$ Gender-responsive, low risk/high strength, and Profile 3 = Aggressive, complex need/low strength. ${ }^{1}$ Aggression refers to the aggression/violence domain. ${ }^{2}$ Skills refers to the Cognitive/social skills domain. ${ }^{3}$ ACEs refers to adverse childhood experiences. 
Women - Profile 1: Low risk/low strength ${ }^{21}$. This profile is defined by having low scores across all dynamic domains (both the dynamic risk domains and the dynamic strength domains). This profile is also defined by having the lowest scores on mental health and childhood adversity. These women are non-aggressive, as evidenced by low scores on the aggressive risk domain.

Women - Profile 2: Gender-responsive, low risk/high strength. This profile is defined as scoring low on all dynamic risk domains, with the exception of mental health concerns, experiences of childhood adversity, and substance use. This profile also scores highest on all dynamic strength domains and is non-aggressive, as evidenced by low scores on the aggressive risk domain.

Women - Profile 3: Aggressive, complex need/low strength. This profile is defined by scoring highest across all dynamic risk domains and gender-responsive needs, such as childhood adversity and mental health concerns — thus making it complex. This profile also scored the highest on criminal history and aggression, indicating that these women may have more violent tendencies and criminality. However, this profile scores low across all dynamic strength domains.

Figure 13 presents the standardized risk and strength domain scores for each of the profiles that emerged for women at Time 1.

\footnotetext{
${ }^{21}$ Importantly, the terms high and low when describing the profiles that emerged do not coincide with the definitions of high and low scores used by the SPIn. Rather, the reference to high and low risk and strength in the profile descriptions is in relation to the scores on each of the domains in comparison to the rest of the sample.
} 


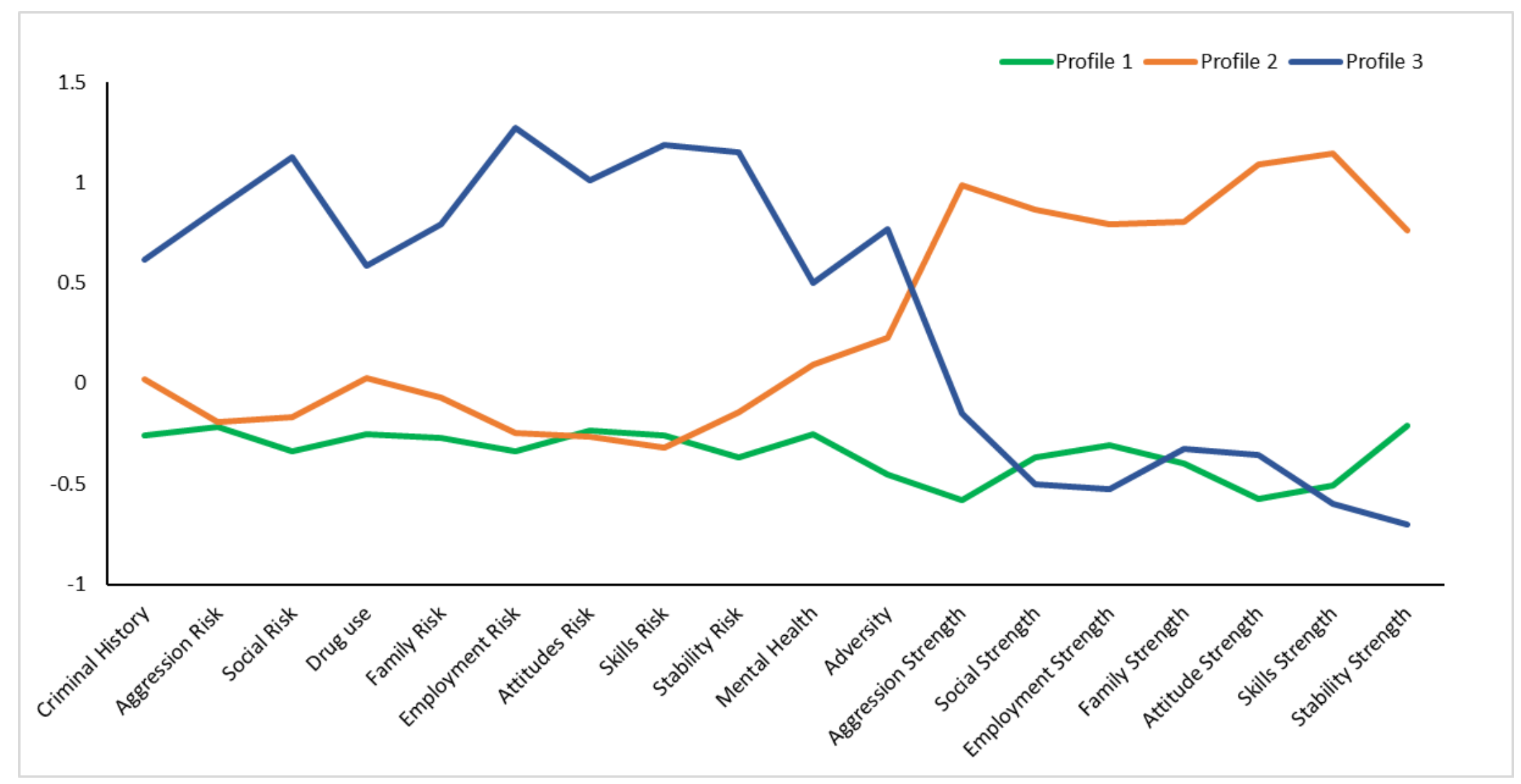

Figure 13. Standardized risk and strength domain scores for the three profiles of women at Time 1. 
Time 1 profile descriptions for men. For the men, the five profiles are labelled as follows: Profile $1=$ Low risk/low strength $(n=1,952 ; 29.2 \%)$, Profile $2=$ Aggressive, complex need/low strength $(n=408 ; 6.1 \%)$, Profile $3=$ Moderate risk/moderate strength $(n=2,244 ; 33.7 \%)$, Profile $4=$ Low risk/high strength $(n=$ $1,718 ; 25.7 \%)$, and Profile $5=$ Low stability, complex need/low strength $(n=353$; $5.3 \%)$. The estimated means and standard deviations for each of the profiles were identified and labelled for men in Table 36. 
Table 36

Means for Each of the Risk and Strength Domains across the Five Profiles of Men at Time 1

\begin{tabular}{lcccccccccc}
\hline & \multicolumn{2}{c}{$\begin{array}{c}\text { Profile 1 } \\
\text { Variables in LPA }\end{array}$} & \multicolumn{2}{c}{$\begin{array}{c}\text { Profile 2 } \\
(n=408)\end{array}$} & $\begin{array}{c}\text { Profile 3 } \\
(n=2,244)\end{array}$ & $\begin{array}{c}\text { Profile 4 } \\
(n=1,718)\end{array}$ & $\begin{array}{c}\text { Profile 5 } \\
(n=353)\end{array}$ \\
\cline { 2 - 9 } & $M$ & $S D$ & $M$ & $S D$ & $M$ & $S D$ & $M$ & $S D$ & $M$ & $S D$ \\
\hline Criminal history & 2.87 & $(3.07)$ & 8.73 & $(5.75)$ & 8.13 & $(5.36)$ & 5.90 & $(5.16)$ & 8.88 & $(5.62)$ \\
Aggression ${ }^{1}$ - Risk & 0.01 & $(0.13)$ & 3.87 & $(1.39)$ & 0.22 & $(0.52)$ & 0.04 & $(0.24)$ & 0.54 & $(0.85)$ \\
Aggression - Strength & 0.23 & $(0.82)$ & 0.14 & $(0.42)$ & 2.01 & $(1.60)$ & 4.04 & $(1.63)$ & 1.50 & $(1.60)$ \\
Substance use - Risk & 3.17 & $(4.54)$ & 8.99 & $(8.87)$ & 7.89 & $(7.53)$ & 5.74 & $(7.15)$ & 9.59 & $(11.97)$ \\
Social influence - Risk & 0.66 & $(1.32)$ & 5.54 & $(4.52)$ & 3.95 & $(3.01)$ & 1.77 & $(2.10)$ & 5.72 & $(3.99)$ \\
Social influence - & 3.18 & $(2.10)$ & 2.96 & $(2.87)$ & 4.20 & $(2.84)$ & 7.81 & $(3.23)$ & 2.44 & $(2.43)$ \\
Strength & & & & & & & & & \\
Family - Risk & 3.13 & $(3.07)$ & 8.85 & $(5.24)$ & 5.50 & $(4.35)$ & 3.23 & $(3.36)$ & 5.81 & $(4.50)$ \\
Family - Strength & 1.88 & $(1.86)$ & 3.00 & $(2.17)$ & 3.72 & $(2.27)$ & 5.95 & $(2.88)$ & 2.33 & $(1.94)$ \\
Employ - Risk & 0.12 & $(0.46)$ & 1.38 & $(2.21)$ & 0.36 & $(0.76)$ & 0.14 & $(0.57)$ & 5.58 & $(2.57)$ \\
Employ - Strength & 1.66 & $(1.16)$ & 3.16 & $(2.81)$ & 3.80 & $(2.64)$ & 6.23 & $(3.05)$ & 0.53 & $(0.91)$ \\
Attitudes - Risk & 0.30 & $(0.68)$ & 5.20 & $(3.83)$ & 1.77 & $(2.00)$ & 0.42 & $(0.98)$ & 2.73 & $(2.72)$ \\
Attitudes - Strength & 2.57 & $(1.60)$ & 2.19 & $(2.62)$ & 4.90 & $(3.09)$ & 10.68 & $(3.70)$ & 3.85 & $(3.53)$ \\
Skills ${ }^{2}$ - Risk & 0.13 & $(0.55)$ & 4.79 & $(3.45)$ & 1.01 & $(1.60)$ & 0.10 & $(0.40)$ & 3.34 & $(2.83)$ \\
Skills - Strength & 1.63 & $(1.35)$ & 0.93 & $(1.61)$ & 2.80 & $(2.48)$ & 8.66 & $(3.36)$ & 1.22 & $(1.81)$ \\
Stability - Risk & 0.54 & $(1.05)$ & 2.19 & $(2.24)$ & 1.62 & $(1.81)$ & 0.76 & $(1.21)$ & 4.51 & $(2.45)$ \\
Stability - Strength & 3.24 & $(1.07)$ & 3.87 & $(1.92)$ & 4.18 & $(1.55)$ & 5.25 & $(1.32)$ & 2.29 & $(1.48)$ \\
Mental health - Flag & 0.44 & $(0.75)$ & 1.21 & $(0.92)$ & 0.87 & $(0.92)$ & 0.63 & $(0.85)$ & 1.43 & $(0.84)$ \\
ACEs ${ }^{3}$ & 0.09 & $(0.33)$ & 1.65 & $(2.61)$ & 1.36 & $(1.41)$ & 0.91 & $(1.24)$ & 1.85 & $(1.61)$ \\
\hline$N$
\end{tabular}

Note. $M=$ Mean, $S D=$ Standard deviation. Profile $1=$ Low risk/low strength, Profile $2=$ Aggressive, complex need/low strength, Profile $3=$ Moderate risk/moderate strength, Profile $4=$ Low risk/high strength, and Profile $5=$ Low stability, complex need/low strength. ${ }^{1}$ Aggression refers to the aggression/violence domain. ${ }^{2}$ Skills refers to the cognitive/social skills domain. ${ }^{3} \mathrm{ACEs}$ refers to adverse childhood experiences. 
Men - Profile 1: Low risk/low strength. This profile is defined by having low scores across all domains, both the dynamic risk and strength domains, as well as mental health, childhood adversity, and criminal history.

Men - Profile 2: Aggressive, complex need/low strength. This profile is defined as having needs across several risk domains, both gender-neutral and genderresponsive, with highest scores on skills risk, attitudes risk, and family risk domains. These men also tend to have experienced childhood experiences and have mental health needs. This profile of men also scores substantially higher than the rest of the men on aggression. Finally, this profile scores low on all strength domains.

Men - Profile 3: Moderate risk/moderate strength. Relative to the other profiles, this profile is defined as scoring moderate on all domains, including dynamic risk and strengths domains, mental health, childhood adversity, and criminal history. Individuals in this profile neither score highest or lowest on any of the domains.

Men - Profile 4: Low risk/high strength. This profile is defined as scoring low on all risk domains, including gender-responsive needs (mental health and childhood adversity); however, this profile scores the highest on all strength domains.

Men - Profile 5: Low stability, complex need/low strength. This profile scores highest among most dynamic risk domains, especially employment risk and stability risk domains. Men in this profile also have gender-responsive needs, such as mental health concerns and experiences of childhood adversity. This profile scores low on aggression as well as all strength domains.

Figure 14 presents the standardized risk and strength domain scores for each of the profiles that emerged for men at Time 1. 


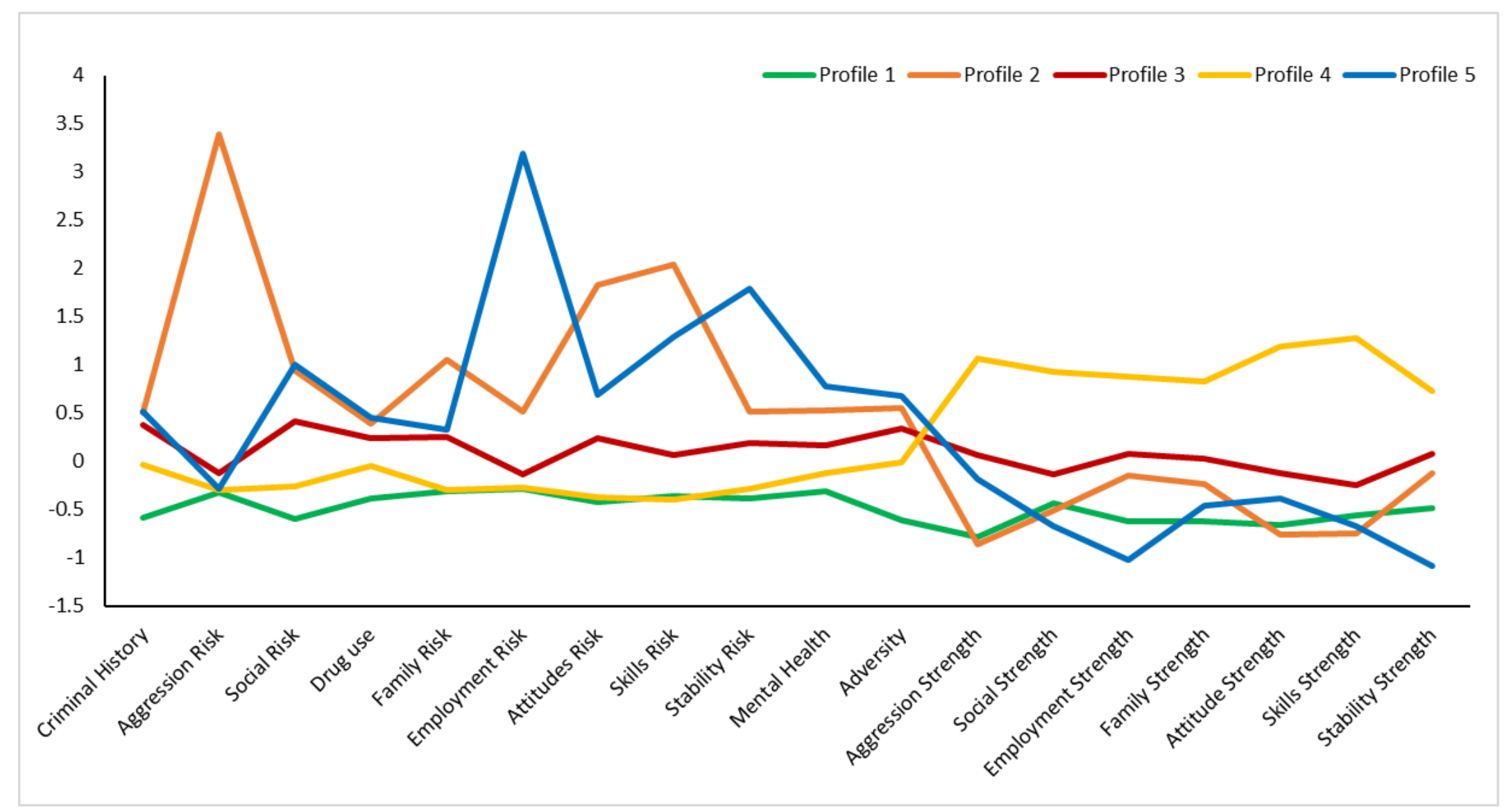

Figure 14. Standardized risk and strength domain scores for the five profiles of men at Time 1. 


\section{LPA Time 2}

Upon considering the model fit indices, theory, and interpretation of the various profiles, it was determined that a 3-structure profile solution fit the data the best for women, whereas a 5-structure profile solution fit the data best for men (see Table 37).

Table 37

Relative Fit Statistics for Time 2 for Men and Women, Independently

\begin{tabular}{lcccccc}
\hline \multicolumn{7}{c}{ Women } \\
\hline Profile & AIC & BIC & ABIC & Entropy & LMR & $\boldsymbol{p}$ \\
\hline 2-Structure & 134898.52 & 135197.11 & 135022.38 & .934 & 4976.62 & $<.001$ \\
3-Structure & $\mathbf{1 3 1 5 6 5 . 1 2}$ & $\mathbf{1 3 1 9 6 6 . 8 6}$ & $\mathbf{1 3 1 7 3 1 . 7 7}$ & $\mathbf{. 9 2 2}$ & $\mathbf{3 3 4 7 . 6 8}$ & $<.001$ \\
4-Structure & 129585.02 & 130089.91 & 129794.46 & .939 & 2003.90 & .579 \\
5-Structure & 128378.31 & 128986.35 & 128630.54 & .927 & 1235.95 & .138 \\
6-Structure & 127328.62 & 128039.81 & 127623.64 & .935 & 1080.04 & .182 \\
\hline & & & Men & & & $\mathbf{p}$ \\
\hline Profile & $\mathbf{A I C}$ & $\mathbf{B I C}$ & $\mathbf{A B I C}$ & $\mathbf{E n t r o p y}$ & $\mathbf{L M R}$ & $\boldsymbol{p}$ \\
\hline 2-Structure & 537567.53 & 537941.86 & 537767.09 & .898 & 16832.69 & $<.001$ \\
3-Structure & 525696.94 & 526200.59 & 525965.44 & .919 & 11837.84 & $<.001$ \\
4-Structure & 519253.61 & 519886.58 & 519591.05 & .936 & 6442.83 & $<.001$ \\
5-Structure & $\mathbf{5 1 4 1 1 3 . 9 1}$ & $\mathbf{5 1 4 8 7 6 . 2 0}$ & $\mathbf{5 1 4 5 2 0 . 2 9}$ & $\mathbf{. 9 0 6}$ & $\mathbf{5 1 4 6 . 9 3}$ & $<.001$ \\
6-Structure & 510588.58 & 511480.18 & 511063.89 & .905 & 3542.16 & .007 \\
\hline
\end{tabular}

Note. AIC $=$ Akaike's Information Criterion. BIC $=$ Bayesian Information Criteria. ABIC $=$ Sample size adjusted Bayesian Information Criteria. LMR $=$ Lo-Mendell-Rubin test.

Posterior profile membership probabilities were also examined (see Table 38).

The probabilities of correct classification for the 3 profiles of women ranged from .94 to .97 , and for the 5 profiles of men ranged from .90 to .99 , which is considered good. 
Table 38

Posterior Profile Membership Probabilities for Time 2 for Men and Women

\begin{tabular}{|c|c|c|c|c|c|}
\hline \multicolumn{6}{|c|}{ Women } \\
\hline \multirow{2}{*}{$\begin{array}{c}\text { Most Likely Profile } \\
\text { Membership }\end{array}$} & \multicolumn{5}{|c|}{ Latent Profiles } \\
\hline & 1 & 2 & 3 & & \\
\hline 1 & .969 & .015 & .016 & & \\
\hline 2 & .044 & .943 & .013 & & \\
\hline 3 & .020 & .007 & .973 & & \\
\hline \multicolumn{6}{|c|}{ Men } \\
\hline Most Likely Profile & \multicolumn{5}{|c|}{ Latent Profiles } \\
\hline Membership & 1 & 2 & 3 & 4 & 5 \\
\hline 1 & .961 & .001 & .037 & .001 & .000 \\
\hline 2 & .003 & .959 & .034 & .000 & .004 \\
\hline 3 & .048 & .006 & .903 & .041 & .002 \\
\hline 4 & .001 & .000 & .053 & .946 & .000 \\
\hline 5 & .000 & .004 & .007 & .001 & .988 \\
\hline
\end{tabular}

Note. Probability of profile membership classification equal to or greater than .70 is considered acceptable.

Time 2 profile descriptions for women and men. The estimated means and standard deviations for each of the profiles were identified and labelled for women (see Table 39) and men (see Table 40). Notably, in both Time 2 and Time 1 the same profiles emerged for women (see descriptions of these profiles of women at Time 1), and for men (see descriptions of profiles for men at Time 1). Although the means and standard deviations changed slightly for profiles across time (for both women and men), the same trends emerged (see Figure 15 and Figure 16). 
Table 39

Means for Each of the Risk and Strength Domains across the Three Profiles of Women at Time 2

\begin{tabular}{|c|c|c|c|c|c|c|}
\hline \multirow[b]{2}{*}{ Variables in LPA } & \multicolumn{2}{|c|}{$\begin{array}{l}\text { Profile 1 } \\
(n=801)\end{array}$} & \multicolumn{2}{|c|}{$\begin{array}{l}\text { Profile } 2 \\
(n=551)\end{array}$} & \multicolumn{2}{|c|}{$\begin{array}{l}\text { Profile } 3 \\
(n=332)\end{array}$} \\
\hline & $M$ & $S D$ & $M$ & $S D$ & $M$ & $S D$ \\
\hline Criminal history & 3.00 & $(3.23)$ & 4.16 & $(4.27)$ & 7.02 & $(5.36)$ \\
\hline Aggression $^{1}-$ Risk & 0.07 & $(0.32)$ & 0.05 & $(0.31)$ & 0.88 & $(1.47)$ \\
\hline Aggression - Strength & 0.71 & $(1.32)$ & 4.23 & $(1.83)$ & 1.81 & $(1.63)$ \\
\hline Substance use - Risk & 3.31 & $(5.05)$ & 5.17 & $(6.39)$ & 8.94 & $(8.00)$ \\
\hline Social influence - Risk & 1.37 & $(2.12)$ & 1.74 & $(2.10)$ & 6.08 & $(4.13)$ \\
\hline Social influence - Strength & 3.36 & $(2.17)$ & 7.75 & $(3.25)$ & 3.09 & $(2.50)$ \\
\hline Family - Risk & 3.22 & $(3.25)$ & 3.83 & $(3.60)$ & 7.56 & $(4.91)$ \\
\hline Family - Strength & 2.61 & $(2.03)$ & 6.16 & $(2.92)$ & 2.90 & $(1.94)$ \\
\hline Employment - Risk & 0.38 & $(0.94)$ & 0.57 & $(1.37)$ & 3.70 & $(3.20)$ \\
\hline Employment - Strength & 1.70 & $(1.51)$ & 4.50 & $(3.18)$ & 0.93 & $(1.36)$ \\
\hline Attitudes - Risk & 0.46 & $(1.07)$ & 0.38 & $(0.90)$ & 2.28 & $(2.48)$ \\
\hline Attitudes - Strength & 3.52 & $(2.31)$ & 11.61 & $(3.76)$ & 4.98 & $(3.57)$ \\
\hline Skills $^{2}-$ Risk & 0.25 & $(0.79)$ & 0.11 & $(0.42)$ & 2.17 & $(2.61)$ \\
\hline Skills - Strength & 2.04 & $(1.74)$ & 8.89 & $(3.61)$ & 2.05 & $(2.41)$ \\
\hline Stability - Risk & 0.92 & $(1.28)$ & 1.34 & $(1.57)$ & 4.29 & $(2.19)$ \\
\hline Stability - Strength & 3.32 & $(1.20)$ & 4.85 & $(1.31)$ & 2.47 & $(1.49)$ \\
\hline Mental health - Flag & 0.87 & $(0.90)$ & 1.22 & $(0.89)$ & 1.58 & $(0.74)$ \\
\hline $\mathrm{ACEs}^{3}$ & 0.64 & $(1.08)$ & 1.84 & $(1.80)$ & 2.83 & $(1.86)$ \\
\hline
\end{tabular}

Note. $M=$ Mean, $S D=$ Standard deviation. Profile $1=$ Low risk/low strength, Profile $2=$ Gender-responsive, low risk/high strength, and Profile 3 $=$ Aggressive, complex need/low strength. ${ }^{1}$ Aggression refers to the aggression/violence domain. ${ }^{2}$ Skills refers to the Cognitive/social skills domain. ${ }^{3} \mathrm{ACEs}$ refers to adverse childhood experiences. 


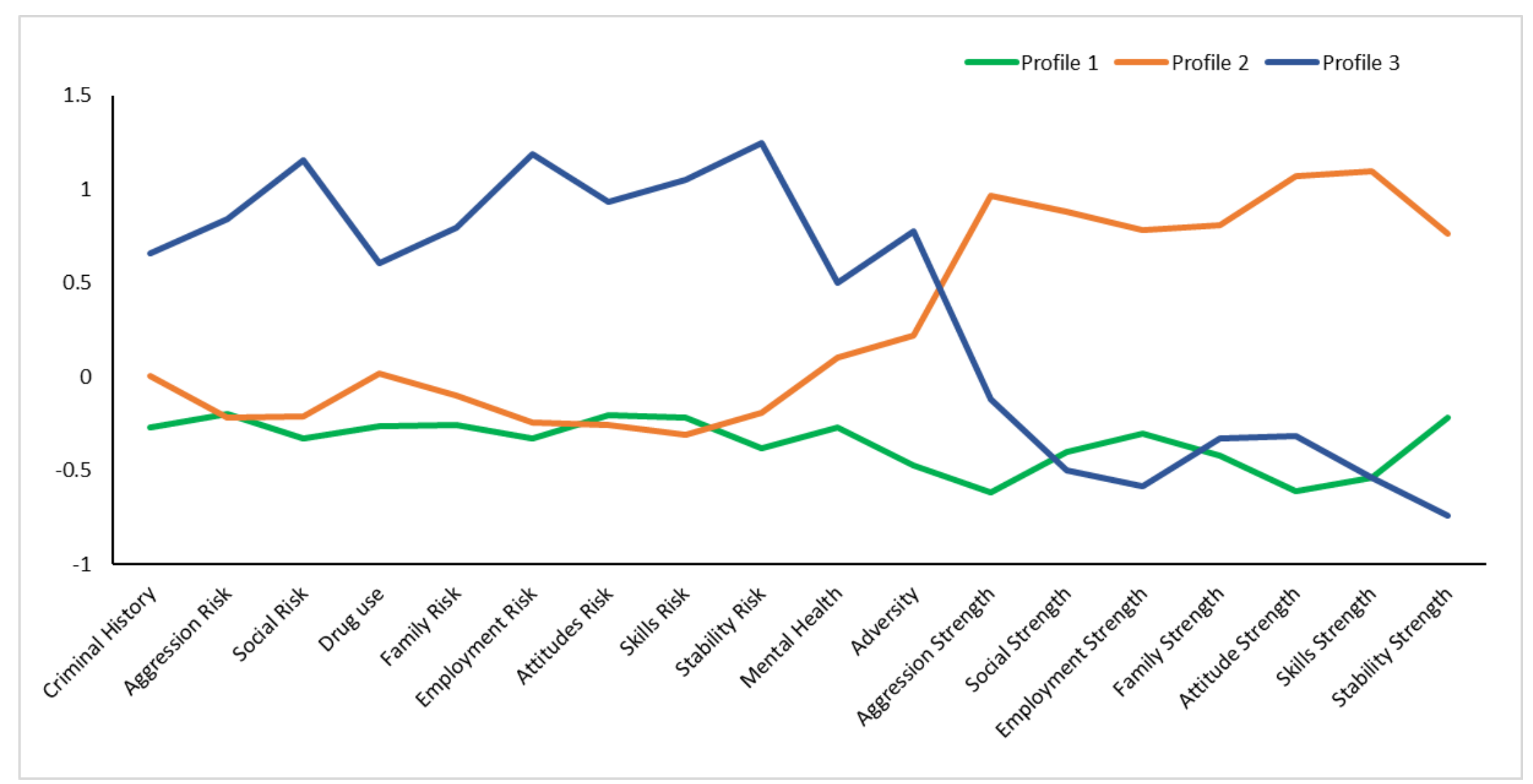

Figure 15. Standardized risk and strength domain scores for the three profiles of women at Time 2. 
Table 40

Means for Each of the Risk and Strength Domains across the Three Profiles of Men at Time 2

\begin{tabular}{|c|c|c|c|c|c|c|c|c|c|c|}
\hline \multirow[b]{2}{*}{ Variables in LPA } & \multicolumn{2}{|c|}{$\begin{array}{c}\text { Profile 1 } \\
(n=1,856)\end{array}$} & \multicolumn{2}{|c|}{$\begin{array}{l}\text { Profile } 2 \\
(n=332)\end{array}$} & \multicolumn{2}{|c|}{$\begin{array}{c}\text { Profile } 3 \\
(n=2,271)\end{array}$} & \multicolumn{2}{|c|}{$\begin{array}{c}\text { Profile } 4 \\
(n=1,843)\end{array}$} & \multicolumn{2}{|c|}{$\begin{array}{l}\text { Profile 5 } \\
(n=373)\end{array}$} \\
\hline & $M$ & $S D$ & $M$ & $S D$ & $M$ & $S D$ & $M$ & $S D$ & $M$ & $S D$ \\
\hline Criminal history & 2.84 & $(3.00)$ & 8.94 & $(5.86)$ & 8.01 & $(5.35)$ & 6.22 & $(5.31)$ & 8.51 & $(5.38)$ \\
\hline Aggression $^{1}-$ Risk & 0.01 & $(0.11)$ & 3.76 & $(1.37)$ & 0.20 & $(0.50)$ & 0.02 & $(0.18)$ & 0.44 & $(0.78)$ \\
\hline Aggression - Strength & 0.23 & $(0.84)$ & 0.11 & $(0.36)$ & 2.07 & $(1.61)$ & 4.11 & $(1.59)$ & 1.57 & $(1.63)$ \\
\hline Substance use - Risk & 2.77 & $(4.17)$ & 7.41 & $(6.97)$ & 6.77 & $(6.72)$ & 5.46 & $(6.84)$ & 8.20 & $(10.05)$ \\
\hline Social $^{2}-$ Risk & 0.61 & $(1.27)$ & 5.40 & $(4.46)$ & 3.71 & $(2.93)$ & 1.73 & $(2.05)$ & 5.42 & $(4.00)$ \\
\hline Social- Strength & 3.17 & $(2.08)$ & 3.14 & $(3.06)$ & 4.32 & $(2.86)$ & 7.88 & $(3.21)$ & 2.82 & $(2.73)$ \\
\hline Family - Risk & 3.05 & $(2.95)$ & 8.85 & $(5.11)$ & 5.54 & $(4.25)$ & 3.19 & $(3.23)$ & 5.61 & $(4.50)$ \\
\hline Family - Strength & 1.90 & $(1.87)$ & 2.88 & $(2.09)$ & 3.75 & $(2.18)$ & 6.12 & $(2.99)$ & 2.54 & $(1.97)$ \\
\hline Employ - Risk & 0.13 & $(0.47)$ & 1.26 & $(1.94)$ & 0.35 & $(0.75)$ & 0.16 & $(0.57)$ & 5.11 & $(2.60)$ \\
\hline Employ - Strength & 1.67 & $(1.14)$ & 3.20 & $(2.64)$ & 3.95 & $(2.68)$ & 6.35 & (3.09) & 0.64 & $(0.97)$ \\
\hline Attitudes - Risk & 0.25 & $(0.58)$ & 5.22 & $(3.80)$ & 1.68 & $(2.00)$ & 0.38 & $(0.90)$ & 2.67 & $(2.66)$ \\
\hline Attitudes - Strength & 2.57 & $(1.59)$ & 2.12 & $(2.57)$ & 5.01 & $(3.09)$ & 10.92 & $(3.65)$ & 4.05 & $(3.66)$ \\
\hline Skills $^{3}-$ Risk & 0.13 & $(0.57)$ & 4.82 & $(3.47)$ & 0.91 & $(1.53)$ & 0.09 & $(0.36)$ & 3.20 & $(2.84)$ \\
\hline Skills - Strength & 1.71 & $(1.38)$ & 0.89 & $(1.55)$ & 2.92 & $(2.56)$ & 8.87 & $(3.34)$ & 1.37 & $(2.03)$ \\
\hline Stability - Risk & 0.50 & $(1.00)$ & 2.23 & $(2.41)$ & 1.51 & $(1.74)$ & 0.77 & $(1.21)$ & 4.26 & $(2.39)$ \\
\hline Stability - Strength & 1.97 & $(1.24)$ & 2.89 & $(1.91)$ & 3.38 & $(1.72)$ & 4.34 & $(1.26)$ & 1.42 & $(1.31)$ \\
\hline Mental health - Flag & 0.44 & $(0.75)$ & 1.21 & $(0.92)$ & 0.86 & $(0.92)$ & 0.66 & $(0.87)$ & 1.41 & $(0.84)$ \\
\hline $\mathrm{ACEs}^{4}$ & 0.09 & $(0.33)$ & 1.58 & (1.63) & 1.32 & $(1.42)$ & 0.97 & $(1.29)$ & 1.71 & $(1.52)$ \\
\hline
\end{tabular}

Note. $M=$ Mean, $S D=$ Standard deviation. Profile $1=$ Low risk/low strength, Profile $2=$ Aggressive, complex need/low strength, Profile $3=$ Moderate risk/moderate strength, Profile $4=$ Low risk/high strength, and Profile $5=$ Low stability, complex need/low strength. ${ }^{1}$ Aggression refers to the aggression/violence domain. ${ }^{2}$ Social refers to social influences domain. ${ }^{3}$ Skills refers to the cognitive/social skills domain. ${ }^{4} \mathrm{ACEs}$ refers to adverse childhood experiences. 


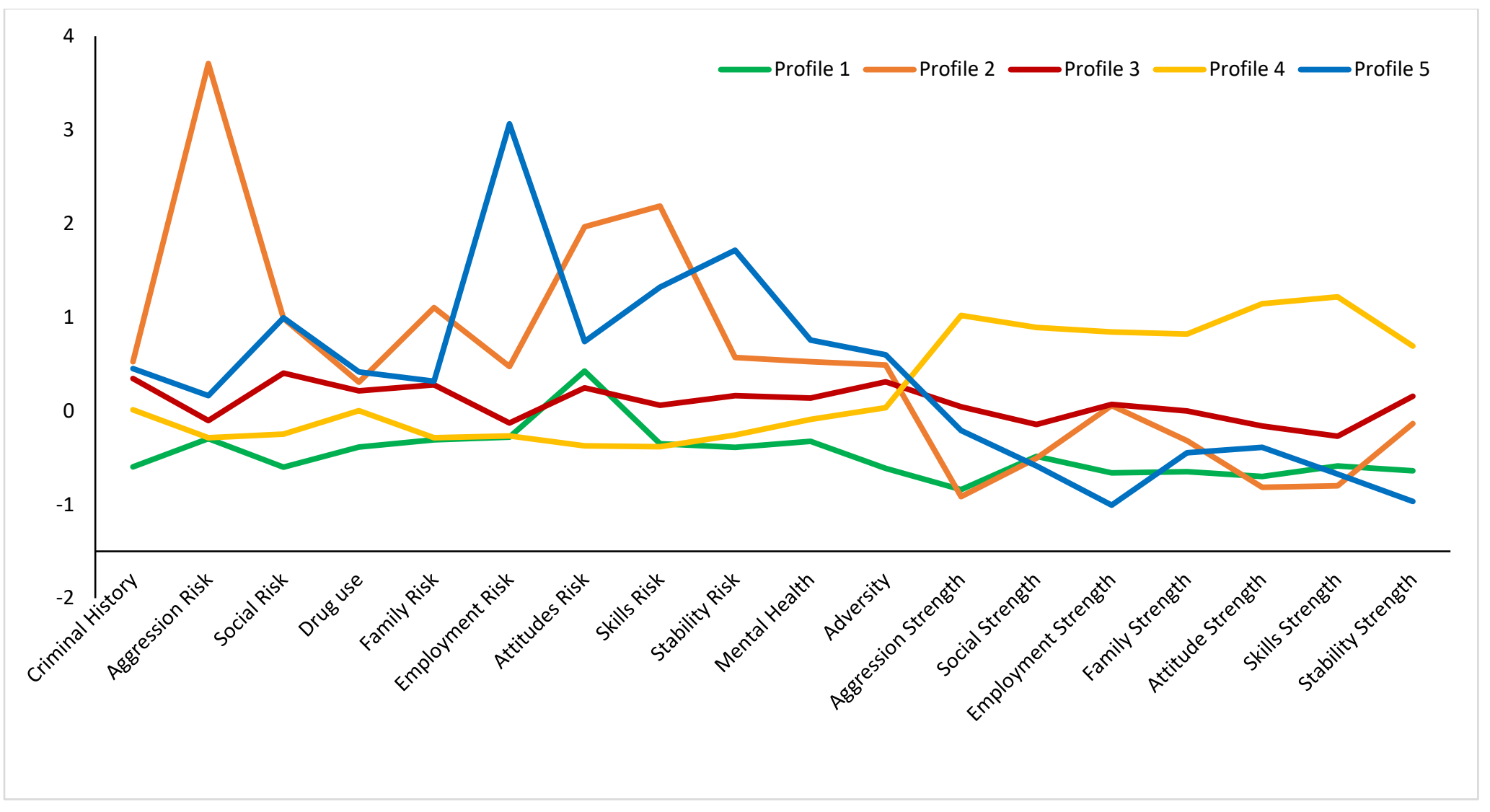

Figure 16. Standardized risk and strength domain scores for the five profiles of men at Time 2. 


\section{LPA Time 3}

Upon considering the model fit indices, theory, and interpretation of the various profiles, it was determined that a 3-structure profile solution fit the data the best for women, whereas a 6-structure profile solution fit the data best for men (see Table 41).

Table 41

Relative Fit Statistics for Time 3 for Men and Women, Independently

\begin{tabular}{|c|c|c|c|c|c|c|}
\hline \multicolumn{7}{|c|}{ Women } \\
\hline Profile & AIC & BIC & ABIC & Entropy & LMR & $p$ \\
\hline 2-Structure & 134676.95 & 134975.54 & 134800.81 & .930 & 4972.02 & $<.001$ \\
\hline 3-Structure & 131357.51 & 131759.25 & 131524.16 & .922 & 3333.82 & .001 \\
\hline 4-Structure & 129383.99 & 129888.88 & 129593.44 & .934 & 1997.37 & .579 \\
\hline 5-Structure & 128074.96 & 128683.00 & 128327.19 & .927 & 1337.56 & .121 \\
\hline 6-Structure & 127087.29 & 127798.47 & 127382.31 & .936 & 1018.46 & .740 \\
\hline \multicolumn{7}{|c|}{ Men } \\
\hline Profile & AIC & BIC & ABIC & Entropy & LMR & $p$ \\
\hline 2-Structure & 534786.27 & 535160.61 & 534985.83 & .895 & 16834.04 & $<.001$ \\
\hline 3-Structure & 523015.19 & 523518.84 & 523283.69 & .919 & 11738.93 & .017 \\
\hline 4-Structure & 516574.57 & 517207.54 & 516912.01 & .937 & 6440.13 & .045 \\
\hline 5-Structure & 511503.16 & 512265.45 & 511909.54 & .904 & 5079.05 & $<.001$ \\
\hline 6-Structure & 507986.36 & $\mathbf{5 0 8 8 7 7 . 9 7}$ & 508461.68 & .905 & 3533.68 & $<.001$ \\
\hline 7-Structure & 507355.14 & 508376.06 & 507899.40 & .915 & 3251.99 & .125 \\
\hline
\end{tabular}

Note. AIC $=$ Akaike's Information Criterion. BIC $=$ Bayesian Information Criteria. ABIC $=$ Sample size adjusted Bayesian Information Criteria. LMR $=$ Lo-Mendell-Rubin test.

Posterior profile membership probabilities were also examined (see Table 42).

The probabilities of correct classification for the 3 profiles of women ranged from .95 to .97 , and for the 6 profiles of men ranged from .89 to .99 , which is considered good. 
Table 42

Posterior Profile Membership Probabilities for Time 3 for Men and Women

\begin{tabular}{ccccccc}
\hline & \multicolumn{5}{c}{ Women } \\
\hline Most Likely Profile & \multicolumn{5}{c}{ Profiles } \\
\cline { 2 - 7 } Membership & 1 & 2 & 3 & \\
\hline 1 & .970 & .016 & .014 & & \\
2 & .027 & .966 & .007 & & \\
3 & .039 & .009 & .951 & & \\
\hline Most Likely Profile & & Men & & & & \\
Membership & 1 & 2 & 3 & 4 & .000 & .031 \\
\hline 1 & .965 & .001 & .003 & .001 & .009 \\
2 & .003 & .987 & .001 & .000 & .000 & .009 \\
3 & .000 & .000 & .911 & .024 & .034 & .031 \\
4 & .000 & .000 & .022 & .960 & .000 & .018 \\
5 & .000 & .000 & .062 & .000 & $\mathbf{. 9 3 8}$ & .000 \\
6 & .006 & .002 & .050 & .052 & .000 & $\mathbf{. 8 9 0}$ \\
\hline
\end{tabular}

Note. Probability of profile membership classification equal to or greater than .70 is considered acceptable.

Time 3 profile descriptions for women. The estimated means and standard deviations for each of the profiles were identified and labelled. Notably, the same profiles emerged across all timepoints (Time 1, Time 2 and Time 3; see descriptions of profiles for women at Time 1), although the means and standard deviations changed slightly across time (See Table 43 and Figure 17). 
Table 43

Means for Each of the Risk and Strength Domains across the Three Profiles of Women at Time 3

\begin{tabular}{|c|c|c|c|c|c|c|}
\hline \multirow[b]{2}{*}{ Variables in LPA } & \multicolumn{2}{|c|}{$\begin{array}{l}\text { Profile 1 } \\
(n=801)\end{array}$} & \multicolumn{2}{|c|}{$\begin{array}{l}\text { Profile } 2 \\
(n=573)\end{array}$} & \multicolumn{2}{|c|}{$\begin{array}{l}\text { Profile } 3 \\
(n=310)\end{array}$} \\
\hline & $M$ & $S D$ & $M$ & $S D$ & $M$ & $S D$ \\
\hline Criminal history & 2.99 & $(3.16)$ & 4.31 & $(4.38)$ & 6.98 & $(5.44)$ \\
\hline Aggression $^{1}-$ Risk & 0.07 & $(0.35)$ & 0.05 & $(0.27)$ & 0.89 & $(1.49)$ \\
\hline Aggression - Strength & 0.76 & $(1.35)$ & 4.25 & $(1.87)$ & 1.87 & $(1.67)$ \\
\hline Substance use - Risk & 3.06 & $(4.88)$ & 5.06 & $(6.10)$ & 8.54 & $(7.41)$ \\
\hline Social influence - Risk & 1.33 & $(2.04)$ & 1.78 & $(2.14)$ & 6.09 & $(4.11)$ \\
\hline Social influence - Strength & 3.47 & $(2.30)$ & 7.66 & $(3.28)$ & 3.20 & $(2.61)$ \\
\hline Family - Risk & 3.23 & $(3.20)$ & 3.84 & $(3.58)$ & 7.71 & $(4.93)$ \\
\hline Family - Strength & 2.69 & $(2.12)$ & 6.11 & $(2.91)$ & 2.92 & $(1.89)$ \\
\hline Employment - Risk & 0.38 & $(0.90)$ & 0.53 & $(1.26)$ & 3.95 & $(3.16)$ \\
\hline Employment - Strength & 1.70 & $(1.54)$ & 4.60 & $(3.20)$ & 0.87 & $(1.37)$ \\
\hline Attitudes - Risk & 0.47 & $(1.06)$ & 0.32 & $(0.78)$ & 2.38 & $(2.49)$ \\
\hline Attitudes - Strength & 3.58 & $(2.31)$ & 11.71 & (3.64) & 4.95 & $(3.76)$ \\
\hline Skills $^{2}-$ Risk & 0.28 & $(0.80)$ & 0.10 & $(0.41)$ & 2.27 & $(2.67)$ \\
\hline Skills - Strength & 2.05 & $(1.69)$ & 8.96 & $(3.65)$ & 1.97 & $(2.32)$ \\
\hline Stability - Risk & 0.96 & $(1.31)$ & 1.33 & $(1.56)$ & 4.27 & $(2.33)$ \\
\hline Stability - Strength & 3.34 & $(1.19)$ & 4.86 & $(1.29)$ & 2.51 & $(1.54)$ \\
\hline Mental health - Flag & 0.88 & $(0.89)$ & 1.22 & $(0.88)$ & 1.61 & $(0.72)$ \\
\hline $\mathrm{ACEs}^{3}$ & 0.70 & $(1.17)$ & 1.86 & $(1.80)$ & 2.69 & $(1.91)$ \\
\hline
\end{tabular}

Note. $M=$ Mean, $S D=$ Standard deviation. Profile 1 = Low risk/low strength, Profile $2=$ Gender-responsive, low risk/high strength, and Profile 3 $=$ Aggressive, complex need/low strength. ${ }^{1}$ Aggression refers to the aggression/violence domain. ${ }^{2}$ Skills refers to the cognitive/social skills domain. ${ }^{3} \mathrm{ACEs}$ refers to adverse childhood experiences. 


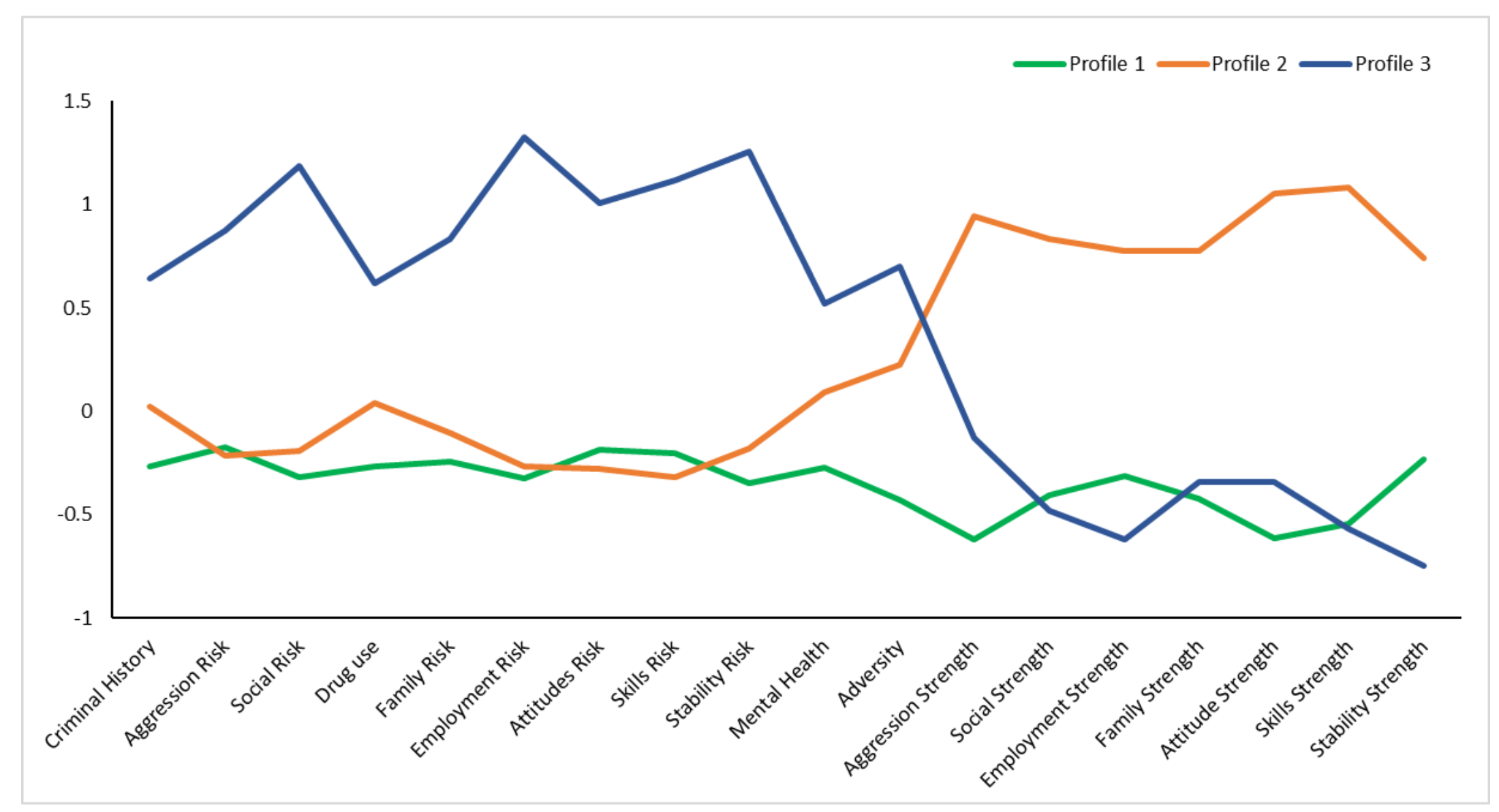

Figure 17. Standardized risk and strength domain scores for the three profiles of women at Time 3. 
Time 3 profile descriptions for men. The estimated means and standard deviations for each of the profiles were identified and labelled. While the same five profiles emerged for Time 1 and Time 2, at Time 3 there were six profiles of men that emerged. Specifically, the six profiles are labelled as follows: Profile $1=$ Low risk/low strength $(n=1,977 ; 29.6 \%)$, Profile $2=$ Aggressive, complex need/low strength $(n=$ $268 ; 4.0 \%)$, Profile $3=$ Moderate risk/moderate strength $(n=2,246 ; 33.6 \%)$, Profile $4=$ Low risk/high strength $(n=875 ; 13.2 \%)$, Profile $5=$ Low stability, complex need/low strength $(n=260 ; 3.9 \%)$, and Profile $6=$ Moderate complex need/low strength $(n=$ $1,049 ; 15.7 \%)$. The estimated means and standard deviations for each of the profiles were identified and labelled for men in Table 44. 
Table 44

Means for Each of the Risk and Strength Domains across the Six Profiles of Men at Time 3

\begin{tabular}{|c|c|c|c|c|c|c|c|c|c|c|c|c|}
\hline \multirow[b]{2}{*}{ Variables in LPA } & \multicolumn{2}{|c|}{$\begin{array}{c}\text { Profile 1 } \\
(n=1,977)\end{array}$} & \multicolumn{2}{|c|}{$\begin{array}{l}\text { Profile } 2 \\
(n=268)\end{array}$} & \multicolumn{2}{|c|}{$\begin{array}{c}\text { Profile } 3 \\
(n=2,246)\end{array}$} & \multicolumn{2}{|c|}{$\begin{array}{l}\text { Profile } 4 \\
(n=875)\end{array}$} & \multicolumn{2}{|c|}{$\begin{array}{l}\text { Profile } 5 \\
(n=260)\end{array}$} & \multicolumn{2}{|c|}{$\begin{array}{c}\text { Profile } 6 \\
(n=1,049)\end{array}$} \\
\hline & $M$ & $S D$ & $M$ & $S D$ & $M$ & $S D$ & $M$ & $S D$ & $M$ & $S D$ & $M$ & $S D$ \\
\hline Criminal history & 3.15 & $(3.34)$ & 9.19 & $(5.89)$ & 6.88 & $(5.25)$ & 5.96 & $(5.35)$ & 8.60 & $(5.55)$ & 9.03 & $(5.40)$ \\
\hline Aggression $^{1}-$ Risk & 0.01 & $(0.10)$ & 3.90 & $(1.32)$ & 0.06 & $(0.29)$ & 0.01 & $(0.12)$ & 0.42 & $(0.79)$ & 0.40 & $(0.67)$ \\
\hline $\begin{array}{l}\text { Aggression - } \\
\text { Strength }\end{array}$ & 0.33 & $(0.93)$ & 0.13 & $(0.40)$ & 3.16 & $(1.54)$ & 4.79 & $(1.60)$ & 1.87 & $(1.67)$ & 1.30 & $(1.40)$ \\
\hline Substances - Risk & 2.81 & (4.19) & 6.96 & $(6.71)$ & 5.52 & $(6.03)$ & 4.77 & $(6.14)$ & 7.62 & $(10.26)$ & 7.57 & $(7.10)$ \\
\hline Social $^{2}$ - Risk & 0.76 & $(1.45)$ & 5.38 & (4.49) & 2.62 & $(2.40)$ & 1.25 & (1.78) & 5.23 & $(4.14)$ & 4.64 & $(3.42)$ \\
\hline Social - Strength & 3.24 & $(2.12)$ & 3.14 & $(3.18)$ & 5.85 & $(3.12)$ & 9.08 & $(3.03)$ & 3.14 & $(3.04)$ & 3.55 & $(2.68)$ \\
\hline Family - Risk & 3.17 & $(3.02)$ & 8.47 & $(5.02)$ & 4.19 & $(3.66)$ & 2.95 & $(3.17)$ & 5.65 & $(4.74)$ & 6.70 & $(4.53)$ \\
\hline Family - Strength & 2.03 & $(1.93)$ & 2.83 & $(2.13)$ & 4.83 & $(2.46)$ & 7.11 & $(3.18)$ & 2.64 & $(2.07)$ & 3.09 & $(2.00)$ \\
\hline Employ - Risk & 0.14 & $(0.48)$ & 1.43 & (2.09) & 0.22 & $(0.65)$ & 0.13 & $(0.52)$ & 6.26 & $(2.34)$ & 0.72 & $(1.04)$ \\
\hline Employ - Strength & 1.85 & $(1.40)$ & 3.10 & $(2.64)$ & 5.07 & $(2.77)$ & 7.36 & $(3.22)$ & 0.47 & $(0.83)$ & 3.17 & $(2.54)$ \\
\hline Attitudes - Risk & 0.33 & $(0.76)$ & 5.40 & $(3.95)$ & 0.80 & $(1.33)$ & 0.22 & $(0.62)$ & 2.50 & $(2.52)$ & 2.66 & $(2.38)$ \\
\hline $\begin{array}{l}\text { Attitudes - } \\
\text { Strength }\end{array}$ & 2.70 & $(1.72)$ & 2.17 & $(2.77)$ & 7.63 & $(3.19)$ & 13.33 & $(2.99)$ & 4.58 & $(3.83)$ & 3.37 & $(2.70)$ \\
\hline Skills $^{3}-$ Risk & 0.12 & $(0.45)$ & 5.02 & $(3.55)$ & 0.26 & $(0.70)$ & 0.03 & $(0.19)$ & 3.19 & $(2.94)$ & 1.87 & $(2.09)$ \\
\hline Skills - Strength & 1.71 & $(1.38)$ & 0.94 & $(1.67)$ & 5.46 & $(2.81)$ & 11.42 & $(2.84)$ & 1.61 & $(2.15)$ & 1.50 & $(1.79)$ \\
\hline Stability - Risk & 0.51 & $(1.02)$ & 2.34 & $(2.44)$ & 0.99 & $(1.36)$ & 0.63 & (1.09) & 4.15 & $(2.49)$ & 2.33 & $(2.08)$ \\
\hline Stability - Strength & 3.40 & (1.13) & 3.73 & (1.98) & 4.90 & $(1.38)$ & 5.56 & (1.18) & 2.59 & $(1.51)$ & 3.72 & $(1.66)$ \\
\hline Mental health & 0.44 & $(0.75)$ & 1.15 & $(0.92)$ & 0.72 & $(0.88)$ & 0.61 & $(0.85)$ & 1.45 & $(0.83)$ & 1.12 & $(0.93)$ \\
\hline $\mathrm{ACEs}^{4}$ & 0.14 & $(0.45)$ & 1.51 & $(1.53)$ & 1.09 & $(1.32)$ & 0.91 & $(1.25)$ & 1.73 & $(1.58)$ & 1.67 & $(1.57)$ \\
\hline
\end{tabular}

Note. $M=$ Mean, $S D=$ Standard deviation. Profile $1=$ Low risk/low strength, Profile $2=$ Aggressive, complex need/low strength, Profile $3=$ Moderate risk/moderate strength, Profile $4=$ Low risk/high strength, Profile $5=$ Low Stability, complex need/low strength, and Profile $6=$ Moderate complex need/low strength. ${ }^{1}$ Aggression refers to the aggression/violence domain. ${ }^{2}$ Social refers to social influences domain. ${ }^{3}$ Skills refers to the cognitive/social skills domain. ${ }^{4} \mathrm{ACEs}$ refers to adverse childhood experiences. 
Men - Profile 1: Low risk/low strength. This profile is defined by having low scores across all dynamic risk and dynamic strength domains, as well as mental health, childhood adversity, and criminal history.

Men - Profile 2: Aggressive, complex need/low strength. This profile is defined as having needs across several risk domains, both gender-neutral and genderresponsive, with highest scores on skills risk, attitudes risk, experienced childhood experiences, and mental health needs. This profile of men also scores substantially higher than the rest of the men on aggression. Finally, this profile scores low on all strength domains.

Men - Profile 3: Moderate risk/moderate strength. Relative to the other profiles, this profile is defined as scoring moderate on all domains, including dynamic risk and strengths domains, mental health, childhood adversity, and criminal history. Individuals in this profile neither score highest or lowest on any of the domains

Men - Profile 4: Low risk/high strength. This profile is defined as scoring low on all risk domains, including gender-responsive needs (mental health and childhood adversity); however, this profile scores substantially higher on all strength domains than any of the other profiles.

Men - Profile 5: Low stability, complex need/low strength. This profile scores moderate to high among all dynamic risk domains, with particularly high scores on the employment risk and stability risk domains. Men in this profile also have genderresponsive needs, such as mental health concerns and experiences of childhood adversity. This profile scores low on aggression as well as all strength domains. 
Men - Profile 6: Moderate complex need/low strength. Men in this profile are characterized as scoring moderately across all gender-neutral risk domains as well as gender-responsive risks, such as childhood adversity and mental health concerns. This profile of men is also non-aggressive and score low on all dynamic strength domains.

Figure 18 presents the standardized risk and strength domain scores for each of the six profiles that emerged for men at Time 3. 


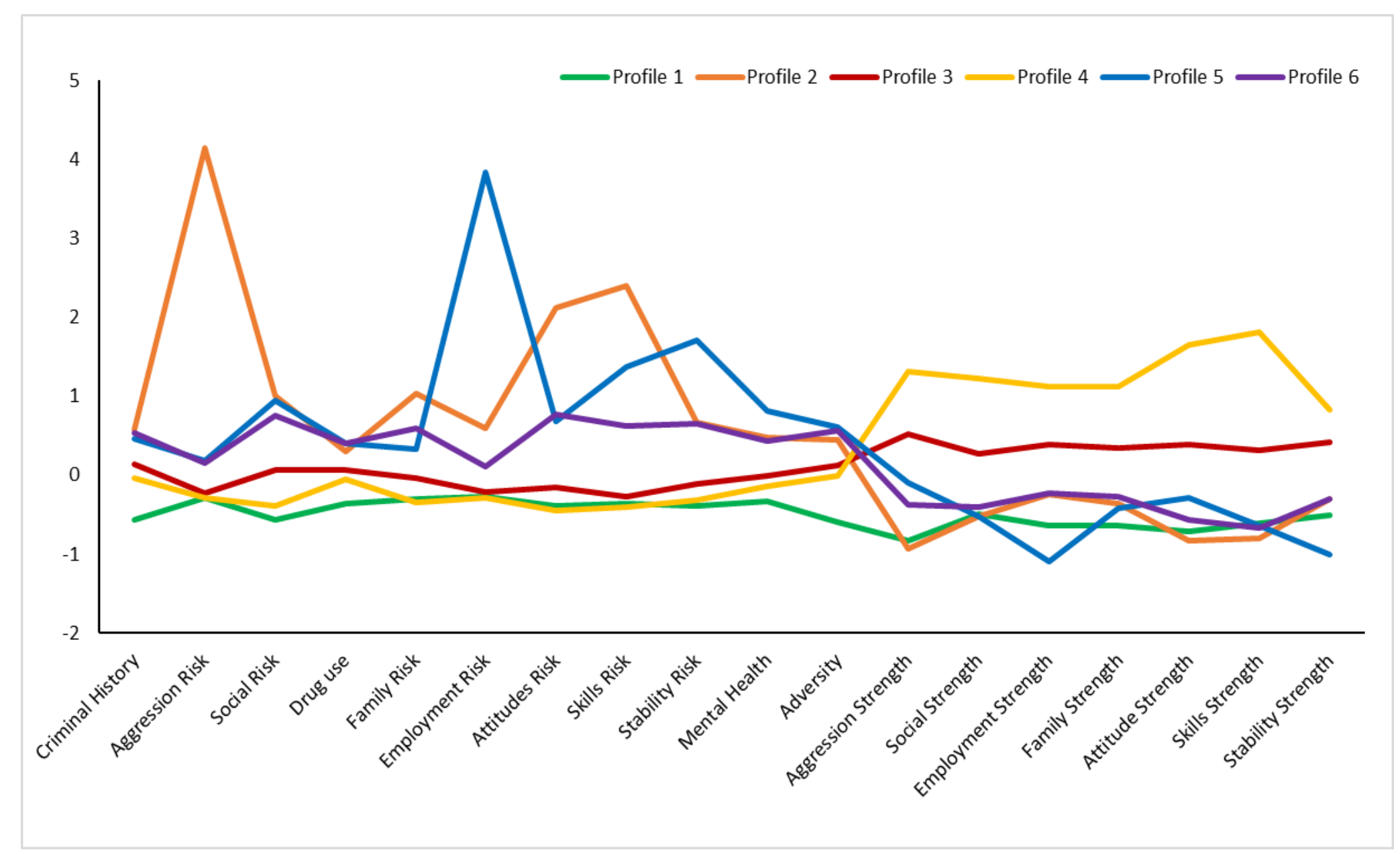

Figure 18. Standardized risk and strength domain scores for the six profiles of men at Time 3. 
Summary of LPA results. For women, three profiles emerged consistently across each timepoint: a low risk/low strength profile, making up the largest proportion of justice-involved women, a gender-responsive, low risk/high strength profile, and an aggressive, complex need/low strength profile, making up the smallest proportion of justice-involved women.

In contrast, for men, five profiles emerged at Time 1 and Time 2; however, at Time 3, a sixth profile emerged. Specifically, at Time 1 and 2, the profiles included: a low risk/low strength profile, an aggressive, complex need/low strength profile, a moderate risk/moderate strength profile, making up the largest proportion of justiceinvolved men, a low risk/high strength profile, and a low stability, complex need/low strength profile, making up the smallest proportion of justice-involved men. In the third timepoint, however, a sixth profile emerged: a moderate complex need/low strength profile.

The results indicated some gender differences and some similarities. For instance, two of the three profiles that emerged for the women also emerged for the men (the low risk/low strength profile, and the aggressive, complex need/low strength profile). The third profile for women that emerged demonstrated a more gender-responsive profile (scoring low on gender-neutral risk factors but higher on more gender-responsive factors such as mental health, childhood adversity, and substance use), which did not emerge among the men. However, there were two profiles of men that emerged that included a mixture of gender-neutral and gender-responsive needs (deemed complex needs when both were present), indicating the importance of considering "gender-responsive" needs for men. Similarly, for both men and women, those who were deemed aggressive, also 
tended to have a mixture of gender-neutral and gender-responsive needs (complex needs), and low strengths. Finally, both men and women had two types of low risk profiles that emerged — one scoring low on strengths and one scoring high on strengths.

\section{Covariate Analyses for Men and Women}

Two covariates were included in the analyses: Age and total static risk scores from the full SPIn assessment. Each covariate will be discussed in turn, for women and men across each of the three timepoints.

Age by classification for women. As seen in Table 1I in Appendix I, there was a significant difference in age between Profile 1 (Low risk/low strength) and Profile 2 (Gender-responsive, low risk/high strength), where women in Profile 2 were found to be older than those in Profile 1 (33.84 vs. 32.98), but only at Time 1 . There were no other significant differences in age among the other pairs of profiles at each of the timepoints (see Tables 2I and 3I in Appendix I). Overall, the mean age was similar for each of the three profiles (see Table 45).

Table 45

Mean Age for Each Profile Across Timepoints for Women

\begin{tabular}{lcccccc}
\hline \multirow{2}{*}{ Profile } & \multicolumn{2}{c}{ Time 1} & \multicolumn{2}{c}{ Time 2 } & \multicolumn{2}{c}{ Time 3 } \\
\cline { 2 - 7 } & $M$ & $S D$ & $M$ & $S D$ & $M$ & $S D$ \\
\hline Profile 1 & 32.98 & $(11.10)$ & 33.40 & $(11.30)$ & 33.49 & $(11.27)$ \\
Profile 2 & 33.84 & $(10.90)$ & 33.70 & $(10.95)$ & 33.64 & $(10.92)$ \\
Profile 3 & 33.43 & $(10.82)$ & 32.61 & $(10.27)$ & 32.44 & $(10.34)$ \\
\hline
\end{tabular}

Note. $M=$ Mean, $S D=$ Standard deviation. Profile $1=$ Low risk/low strength, Profile $2=$ Gender-responsive, low risk/high strength, and Profile $3=$ Aggressive, complex need/low strength. 
Age by classification for men. As seen in Table 4I, 5I, and 6I in Appendix I, there were no significant differences in age among the different profiles that emerged at each of the timepoints. The mean age was similar for each of the profiles and this did not change over time (see Table 46). This indicates that age did not inform the typology formation for men.

Table 46

Mean Age for Each Profile Across Timepoints for Men

\begin{tabular}{lcccccc}
\hline \multirow{2}{*}{ Profile } & \multicolumn{2}{c}{ Time 1 } & \multicolumn{2}{c}{ Time 2 } & \multicolumn{2}{c}{ Time 3 } \\
\cline { 2 - 7 } & $M$ & $S D$ & $M$ & $S D$ & $M$ & $S D$ \\
\hline Profile 1 & 33.84 & $(11.99)$ & 33.82 & $(12.03)$ & 33.79 & $(11.94)$ \\
Profile 2 & 34.94 & $(10.67)$ & 34.97 & $(10.64)$ & 35.36 & $(11.22)$ \\
Profile 3 & 34.62 & $(11.34)$ & 34.82 & $(11.35)$ & 34.39 & $(11.31)$ \\
Profile 4 & 34.66 & $(11.42)$ & 34.37 & $(11.26)$ & 34.77 & $(11.18)$ \\
Profile 5 & 33.99 & $(12.77)$ & 34.10 & $(13.18)$ & 34.52 & $(13.49)$ \\
Profile 6 & -- & -- & -- & -- & 34.90 & $(11.46)$ \\
\hline
\end{tabular}

Note. $M=$ Mean, $S D=$ Standard deviation. At Time 1 and Time 2: Profile $1=$ Low risk/low strength, Profile 2 = Aggressive, complex need/low strength, Profile $3=$ Moderate risk/moderate strength, Profile $4=$ Low risk/high strength, and Profile $5=$ Low stability, complex need/low strength. At Time 3, Profiles 1-5 were similar to Time 2 however, a new profile emerged: Profile $6=$ Moderate complex need/low strength.

Total static risk scores by classification for women. Total static risk scores were significantly different across each of the three profiles and across each of the three timepoints (see Tables 7I, 8I, and 9I in Appendix I). For instance, total static risk scores were lowest among women in Profile 1 (Low risk/low strength), and were highest among Profile 3 (Aggressive, complex need/low strength), which was consistent across each timepoint (see Table 47). These results were not surprising, given that women in Profile 3 
had highest dynamic risk scores and scored highest on aggression, in comparison to the rest of the women.

Table 47

Mean Total Static Risk Scores for Each Profile Across Timepoints for Women

\begin{tabular}{lcccccc}
\hline \multirow{2}{*}{ Profile } & \multicolumn{2}{c}{ Time 1 } & \multicolumn{2}{c}{ Time 2 } & \multicolumn{2}{c}{ Time 3 } \\
\cline { 2 - 7 } & $M$ & $S D$ & $M$ & $S D$ & $M$ & $S D$ \\
\hline Profile 1 & 12.36 & $(10.12)$ & 11.84 & $(9.89)$ & 12.17 & $(10.09)$ \\
Profile 2 & 18.97 & $(13.64)$ & 18.47 & $(13.12)$ & 18.92 & $(13.46)$ \\
Profile 3 & 33.40 & $(16.20)$ & 33.49 & $(15.76)$ & 33.96 & $(15.60)$ \\
\hline
\end{tabular}

Note. $M=$ Mean, $S D=$ Standard deviation. Profile $1=$ Low risk/low strength, Profile $2=$ Gender-responsive, low risk/high strength, and Profile $3=$ Aggressive, complex need/low strength.

Total static risk scores by classification for men. Total static risk scores were significantly different across most pairs of profiles. At Time 1, Profile 2 (Aggressive, complex need/low strength) and Profile 5 (Low stability, complex need/low strength) did not significantly differ in terms of total static risk scores (40.33 vs. 38.23 , respectively). However, the total static risk scores significantly differed across all other profile comparisons (See Table 10I in Appendix I). At Time 2, total static risk scores for Profile 3 (Moderate risk/moderate strength) and Profile 4 (Low risk/high strength) did not significantly differ (28.21 vs. 20.48, respectively). However, the total static risk scores significantly differed across all other profile comparisons (see Table 11I in Appendix I). Finally, at Time 3, total static risk scores significantly differed across all profiles, with the exception of Profile 5 (Low stability, complex need/low strength) and Profile 6 (Moderate complex need/low strength), where total static risk scores were similar (36.73 vs. 35.12, respectively; see Table 12I in Appendix I). Across each timepoint, Profile 2 
was found to have the highest total static risk score and Profile 1 was found to have the lowest total static risk score (See Table 48).

Table 48

Mean Total Static Risk Score for Each Profile Across Timepoints for Men

\begin{tabular}{lcccccc}
\hline \multirow{2}{*}{ Profile } & \multicolumn{2}{c}{ Time 1 } & \multicolumn{2}{c}{ Time 2 } & \multicolumn{2}{c}{ Time 3 } \\
\cline { 2 - 7 } & $M$ & $S D$ & $M$ & $S D$ & $M$ & $S D$ \\
\hline Profile 1 & 10.25 & $(6.99)$ & 9.92 & $(6.97)$ & 10.92 & $(8.15)$ \\
Profile 2 & 40.33 & $(19.72)$ & 40.15 & $(19.88)$ & 41.50 & $(20.16)$ \\
Profile 3 & 29.41 & $(15.92)$ & 28.21 & $(15.80)$ & 23.23 & $(14.53)$ \\
Profile 4 & 19.55 & $(14.22)$ & 20.48 & $(15.03)$ & 19.13 & $(14.93)$ \\
Profile 5 & 38.23 & $(18.64)$ & 36.30 & $(18.08)$ & 36.73 & $(17.69)$ \\
Profile 6 & -- & -- & -- & -- & 35.12 & $(16.84)$ \\
\hline
\end{tabular}

Note. $M=$ Mean, $S D=$ Standard deviation. At Time 1 and Time 2: Profile $1=$ Low risk/low strength, Profile $2=$ Aggressive, complex need/low strength, Profile $3=$ Moderate risk/moderate strength, Profile $4=$ Low risk/high strength, and Profile $5=$ Low stability, complex need/low strength. At Time 3, Profiles 1-5 were similar to Time 2 however, another profile emerged: Profile $6=$ Moderate complex need/low strength.

\section{Auxiliary Analyses}

Women at Time 1. Indigenous status was assessed as an auxiliary variable.

Based on an omnibus chi-square test, there were significant differences between profiles, in terms of the proportions of women who were Indigenous and non-Indigenous, $\chi^{2}(2)=$ $18.59, p<.001$. In fact, there were significant differences in the Indigenous distributions between all pairs of profiles (see Table $1 \mathrm{~J}$ in Appendix $\mathrm{J}$ ).

As seen in Figure 19 and Table 49, 27.52\% of all Indigenous women were in Profile 3 (Aggressive, complex need /low strength), whereas only $16.63 \%$ of all nonIndigenous women were in this profile $\left(\chi^{2}=23.56, p<.001\right)$. In contrast, $51.83 \%$ of all 
non-Indigenous women were in Profile 1 (Low risk/low strength), and only $38.82 \%$ of all Indigenous women were in this profile $\left(\chi^{2}=20.94, p<.001\right)$.

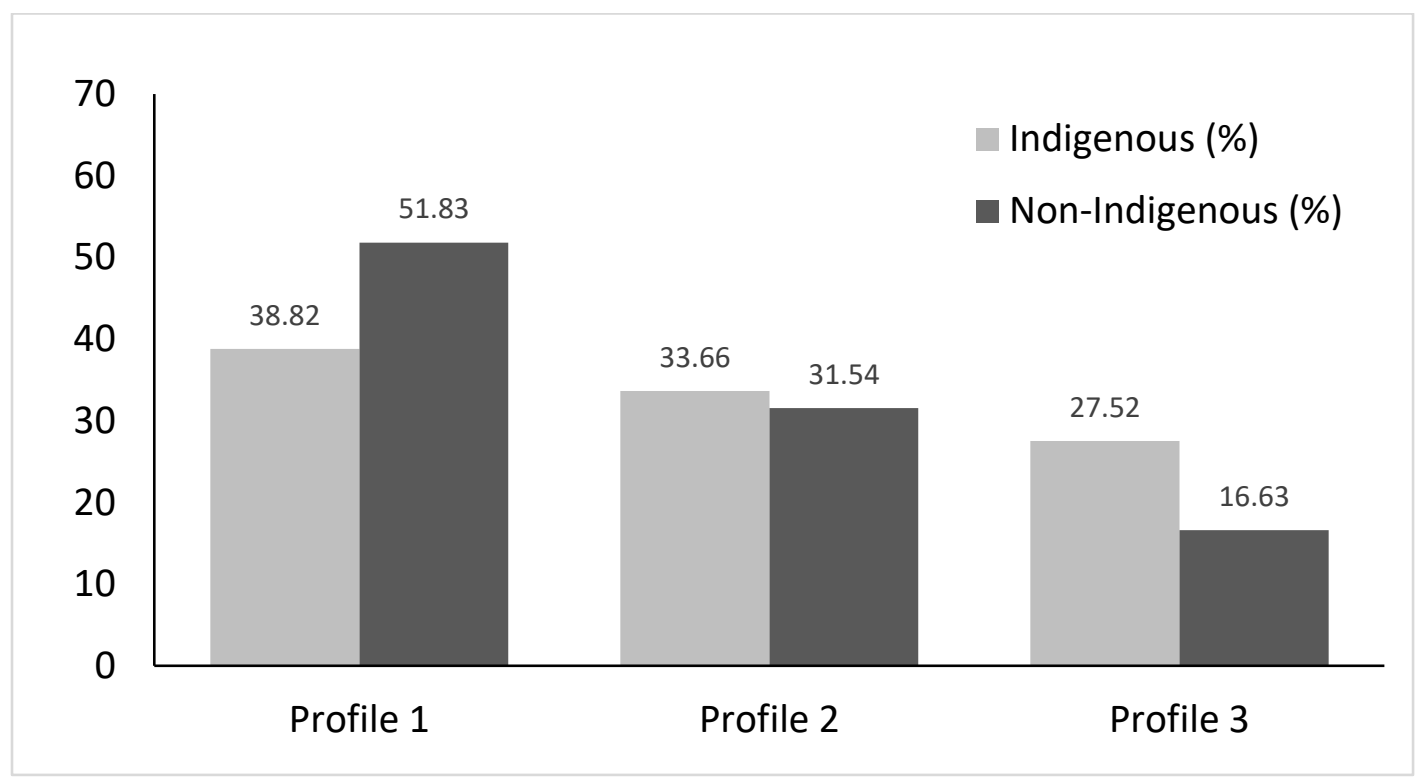

Figure 19. Proportion of Indigenous and non-Indigenous women in each profile at Time 1.

Table 49

Time 1 Proportions of Indigenous and Non-Indigenous Women in Each Profile

\begin{tabular}{lccrr}
\hline Profile & $\begin{array}{c}\text { Percentage of } \\
\text { Indigenous women }\end{array}$ & $\begin{array}{c}\text { Percentage of non- } \\
\text { Indigenous women }\end{array}$ & \multicolumn{1}{c}{$\chi^{2}$} & \multicolumn{1}{c}{$p$} \\
\hline Profile 1 & 38.82 & $\mathbf{5 1 . 8 3}$ & 20.94 & $<.001$ \\
Profile 2 & 33.66 & 31.54 & 0.85 & .358 \\
Profile 3 & $\mathbf{2 7 . 5 2}$ & 16.63 & 23.56 & $<.001$ \\
\hline
\end{tabular}

Note. Profile $1=$ Low risk/low strength, Profile $2=$ Gender-responsive, low risk/high strength, and Profile $3=$ Aggressive, complex need/low strength. $\chi^{2}=$ Chi-square test. 
Women at Time 2 and Time 3. Similar to Time 1, there were significant differences in the Indigenous distributions between all pairs of profiles at Time $2, \chi^{2}(2)=$ 41.38, $p<.001$, and at Time 3, $\chi^{2}(2)=34.52, p<.001$ (see Table $1 \mathrm{~J}$ in Appendix J). Upon examining the number of Indigenous women in each profile in relation to total number of Indigenous women, Time 2 and Time 3 demonstrated that approximately one third of Indigenous women were in each of the three profiles. Notably, similar to Time 1, a higher proportion of all Indigenous women were in Profile 3 (Aggressive, complex need/low strength), in comparison to the proportion of all non-Indigenous women at both Time $2\left(\chi^{2}=46.59, p<.001\right)$, and Time $3\left(\chi^{2}=49.65, p<.001\right)$. In contrast, a higher proportion of all non-Indigenous women were in Profile 1 (Low risk/low strength), in comparison to the proportion of all Indigenous women at both Time $2\left(\chi^{2}=27.30, p<\right.$ $.001)$, and Time $3\left(\chi^{2}=27.30, p<.001\right)$. See Table 50 for the proportions of Indigenous and non-Indigenous women in each profile for Time 2 and Time 3. 
Table 50

Time 2 and 3 Proportions of Indigenous and Non-Indigenous Women in Each Profile

\begin{tabular}{lccrr}
\hline \multicolumn{5}{c}{ Time 2 } \\
\hline Profile & $\begin{array}{c}\text { Percentage of } \\
\text { Indigenous women }\end{array}$ & $\begin{array}{c}\text { Percentage of non- } \\
\text { Indigenous women }\end{array}$ & $\chi^{2}$ & $p$ \\
\hline Profile 1 & 36.23 & $\mathbf{5 1 . 1 3}$ & 27.30 & $<.001$ \\
Profile 2 & 32.26 & 32.87 & 0.05 & .821 \\
Profile 3 & $\mathbf{3 1 . 5 1}$ & 16.00 & 46.59 & $<.001$ \\
\hline & & Time 3 & & \\
\hline & Percentage of & Percentage of non- & \\
\hline Profile & Indigenous women & Indigenous women & $\chi^{2}$ & .001 \\
Profile 1 & 36.23 & $\mathbf{5 1 . 1 3}$ & 27.30 & $<.001$ \\
Profile 2 & 33.50 & 34.19 & 49.65 & $<.001$ \\
\hline
\end{tabular}

Note. Profile $1=$ Low risk/low strength, Profile $2=$ Gender-responsive, low risk/high strength, and Profile $3=$ Aggressive, complex need/low strength. $\chi^{2}=$ Chi-square test.

Men at Time 1. Based on an omnibus chi-square test, there were significant differences between profiles, in terms of the proportions of men who were Indigenous and non-Indigenous, $\chi^{2}(4)=76.67, p<.001$. Upon further examination, it was found that there were no significant differences in the Indigenous distributions between Profile 2 and Profile $4, \chi^{2}(1)=.06, p=.806$, or between Profile 3 and Profile $5, \chi^{2}(1)=3.65, p=$ .056. The Indigenous distribution comparisons for each of the other profiles were significantly different (see Table 2J in Appendix J).

As seen in Figure 20 and Table 51, of all Indigenous men, almost half (45.91\%) were in Profile 3 (Moderate risk/moderate strength), whereas only $31.78 \%$ of all nonIndigenous men were in this profile $\left(\chi^{2}=67.41, p<.001\right)$. Similarly, a higher proportion of the Indigenous men were in Profile 5 (Low stability, complex need/low strength) than 
non-Indigenous men (9.34 vs. $\left.4.68 ; \chi^{2}=32.70, p<.001\right)$. In contrast, a higher proportion of non-Indigenous men were in Profile 1 (Low risk/low strength) than Indigenous men $\left(31.47 \%\right.$ vs. $\left.14.30 \% ; \chi^{2}=107.50, p<.001\right)$. Similar proportions of Indigenous and nonIndigenous men were in Profile 2 (Aggressive, complex need/low strength) and Profile 4 (Low risk/high strength).

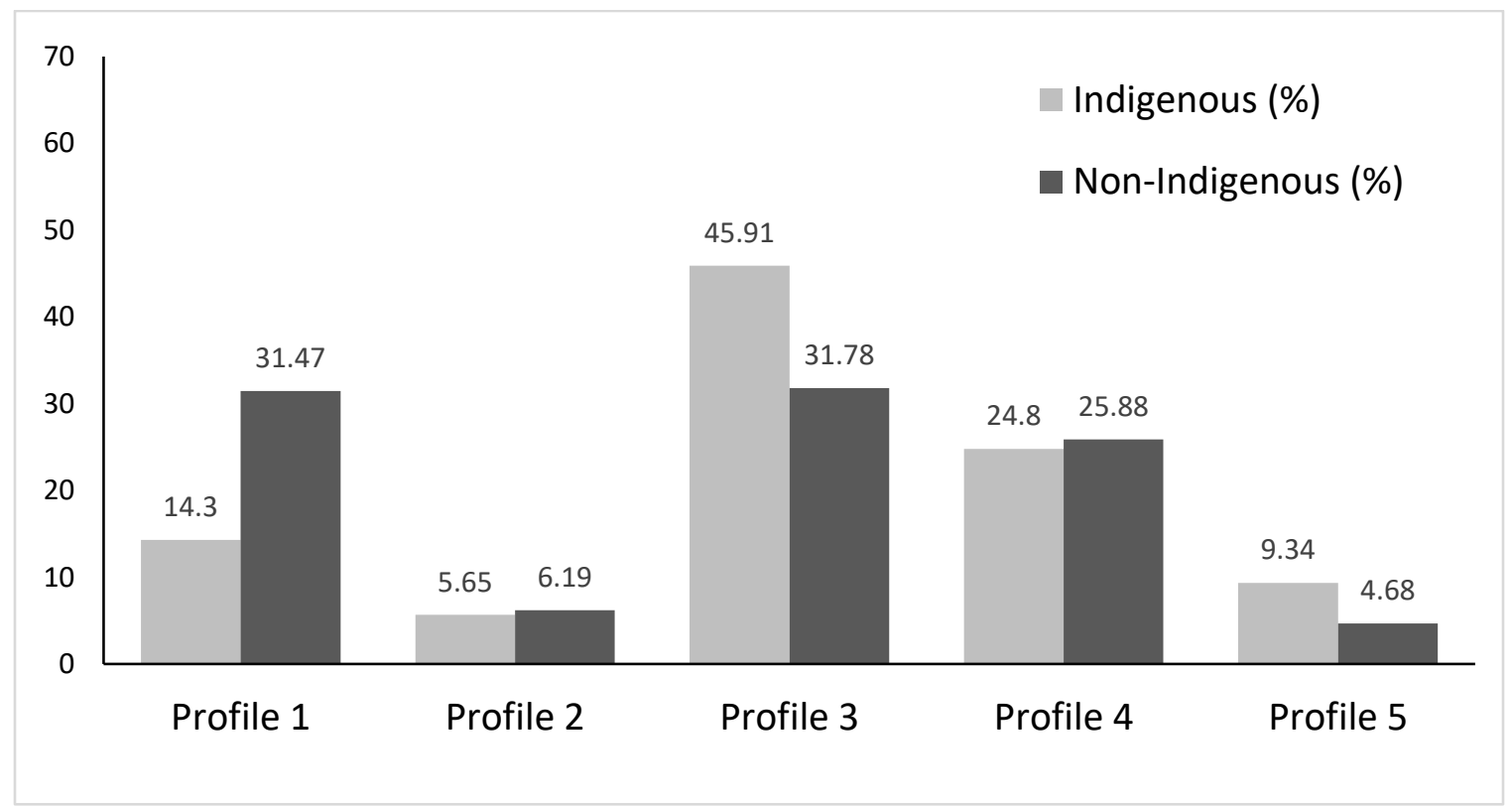

Figure 20. Proportions of Indigenous and non-Indigenous men in each profile at Time 1. 
Table 51

Time 1 Proportions of Indigenous and Non-Indigenous Men in Each Profile

\begin{tabular}{lccrr}
\hline Profile & $\begin{array}{c}\text { Percentage of } \\
\text { Indigenous men }\end{array}$ & $\begin{array}{c}\text { Percentage of non- } \\
\text { Indigenous men }\end{array}$ & \multicolumn{1}{c}{$\chi^{2}$} & \multicolumn{1}{c}{$p$} \\
\hline Profile 1 & 14.30 & $\mathbf{3 1 . 4 7}$ & 107.50 & $<.001$ \\
Profile 2 & 5.65 & 6.19 & 0.37 & .544 \\
Profile 3 & $\mathbf{4 5 . 9 1}$ & 31.78 & 67.41 & $<.001$ \\
Profile 4 & 24.80 & 25.88 & 0.46 & .497 \\
Profile 5 & $\mathbf{9 . 3 4}$ & 4.68 & 32.70 & $<.001$ \\
\hline
\end{tabular}

Note. Profile $1=$ Low risk/low strength, Profile $2=$ Aggressive, complex need/low strength, Profile 3 = Moderate risk/moderate strength, Profile $4=$ Low risk/high strength, and Profile $5=$ Low stability, complex need/low strength. $\chi^{2}=$ Chi-square test.

Men at Time 2 and Time 3. Similar to Time 1, there were significant differences between profiles in terms of the proportions of men who were Indigenous and nonIndigenous at Time $2, \chi^{2}(4)=100.13, p<.001$, and at Time $3, \chi^{2}(4)=96.65, p<.001$ (see Table 3J in Appendix J). At Time 2, however, it was found that there were no significant differences in the Indigenous distributions between Profile 2 and Profile 3, $\chi^{2}(1)=2.08, p=.150$, or between Profile 2 and Profile $4, \chi^{2}(1)=0.11, p=.736$. Notably, since there was an additional profile that emerged at Time 3, there were some differences at this timepoint. While similar to Time 2 there were no significant differences in Indigenous distributions between Profile 2 and Profile $3, \chi^{2}(1)=0.45, p=.505$, or between Profile 2 and Profile $4, \chi^{2}(1)=0.27, p=.605$, there were also no significant differences between Profile 3 and Profile $4, \chi^{2}(1)=3.50, p=.061$, or between Profile 5 and Profile 6, $\chi^{2}(1)=1.95, p=.162$ (see Table 3J in Appendix J). 
Upon examining the number of Indigenous men in each profile in relation to the total number of Indigenous men, Time 2 and Time 3 demonstrated that the largest proportion of Indigenous men were in Profile 3 (Moderate risk/moderate strength). As seen in Table 52, of all Indigenous men, at Time 2 almost half (43.95\%) were in Profile 3 (Moderate risk/moderate strength), whereas 32.54\% of all non-Indigenous men were in this profile $\left(\chi^{2}=43.70, p<.001\right)$. At Time 3 , however, $38.52 \%$ of Indigenous men were in Profile 3, whereas $32.92 \%$ of all non-Indigenous men were in this profile $\left(\chi^{2}\right.$ $=10.61, p<.001)$. The magnitude of the difference between these profiles decreased from Time 1 to Time 3. In contrast, just under a third of all non-Indigenous men were in Profile 1 (Low risk/low strength), which was larger than the proportion of Indigenous men at Time $2\left(29.89\right.$ vs. $\left.13.84 ; \chi^{2}=96.80, p<.001\right)$ and at Time $3\left(31.74\right.$ vs. $15.46 ; \chi^{2}=$ 95.87, $p<.001)$. Given that Time 3 had an additional profile that emerged, Figure 21 demonstrates the proportion of all Indigenous and all non-Indigenous men across the six profiles. 
Table 52

Time 2 and 3 Proportions of Indigenous and Non-Indigenous Men in Each Profile

\begin{tabular}{lccrr}
\hline \multicolumn{5}{c}{ Time 2 } \\
\hline Profile & $\begin{array}{c}\text { Percentage of } \\
\text { Indigenous men }\end{array}$ & $\begin{array}{c}\text { Percentage of non- } \\
\text { Indigenous men }\end{array}$ & \multicolumn{1}{c}{$\chi^{2}$} & \multicolumn{1}{c}{$p$} \\
\hline Profile 1 & 13.84 & $\mathbf{2 9 . 8 9}$ & 96.80 & $<.001$ \\
Profile 2 & 5.19 & 4.94 & 0.10 & .753 \\
Profile 3 & $\mathbf{4 3 . 9 5}$ & 32.54 & 43.70 & $<.001$ \\
Profile 4 & 27.68 & 27.60 & 0.01 & .960 \\
Profile 5 & $\mathbf{9 . 3 4}$ & 5.03 & 26.62 & $<.001$ \\
\hline \multicolumn{5}{c}{ Time 3 } \\
\hline Profile & Percentage of & Percentage of non- & \\
\hline Profile 1 & Indigenous men & Indigenous men & $\chi^{2}$ & $p$ \\
Profile 2 & 15.46 & $\mathbf{3 1 . 7 4}$ & 95.87 & $<.001$ \\
Profile 3 & 4.04 & 4.01 & 0.01 & .998 \\
Profile 4 & $\mathbf{3 8 . 5 2}$ & 32.92 & 10.61 & .001 \\
Profile 5 & 12.11 & 13.26 & 0.87 & .351 \\
Profile 6 & $\mathbf{6 . 9 2}$ & 3.44 & 24.36 & $<.001$ \\
\hline
\end{tabular}

Note. Time 2: Profile $1=$ Low risk/low strength, Profile $2=$ Aggressive, complex need/low strength, Profile $3=$ Moderate risk/moderate strength, Profile $4=$ Low risk/high strength, and Profile $5=$ Low stability, complex need/low strength. Time 3: Profile $1=$ Low risk/low strength, Profile 2 = Aggressive, complex need/low strength, Profile $3=$ Moderate risk/moderate strength, Profile $4=$ Low risk/high strength, Profile $5=$ Low stability, complex need/low strength, and Profile $6=$ Moderate complex need/low strength. $\chi^{2}=$ Chi-square test. 


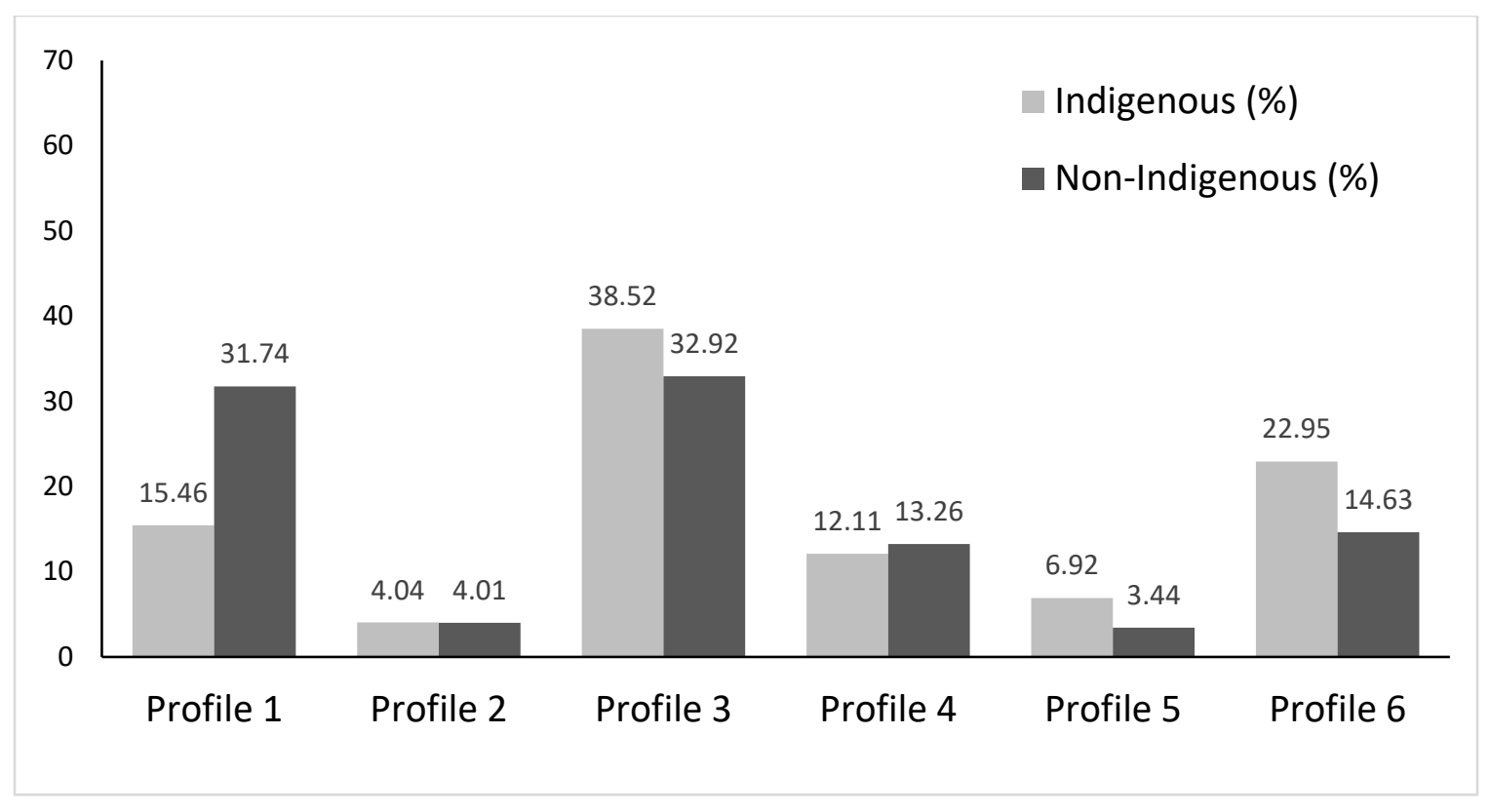

Figure 21. Proportions of Indigenous and non-Indigenous men in each profile at Time 3.

Summary of covariate and auxiliary analyses. SPIn Full Assessment total static risk scores were found to inform typology formation for both genders. Specifically, for women, there were significant differences in total static risk scores among all profiles, with Profile 1 (Low risk/low strength) scoring the lowest, followed by Profile 2 (Genderresponsive, low risk/high strength), and then Profile 3 (Aggressive, complex need/low strength) scoring the highest across each timepoint. In contrast, for men, the total static risk scores were found to be significantly different among all profiles, with the exception of Profile 2 (Aggressive, complex need/low strength) and Profile 5 (Low stability, complex need/low strength) which are found to have similar mean total static risk scores at Time 1 and Time 2. At Time 3, once again there were significant differences in total static risk scores among all profiles with the exception of Profile 5 (Low stability, complex need/low strength) and Profile 6 (Moderate complex need/low strength), which were found to have similar total static risk scores. Taken together, this indicates that static 
risk scores informed the typology formation for men and women. Furthermore, across each of the timepoints, results of the covariate analysis found that age did not inform the typology formation for both men and women, with the exception of Time 1 for the women whereby those in Profile 2 were found to be older than those in Profile 1.

One auxiliary variable was examined - Indigenous status. For women, about a third of Indigenous women were in each profile, across each timepoint. The proportion of non-Indigenous women was higher than the proportion of Indigenous women in Profile 1 (Low risk/low strength). Similar proportions of Indigenous and non-Indigenous women were in Profile 2 (Gender-responsive, low risk/high strength). Finally, the proportion of Indigenous women was higher than the proportion of non-Indigenous women in Profile 3 (Aggressive, complex need/low strength). This pattern was found among each of the timepoints.

In comparison, for men, a significantly higher proportion of non-Indigenous men were in Profile 1 (Low risk/low strength) in comparison to the proportion of Indigenous men. In contrast, a significantly higher proportion of Indigenous men were in Profile 3 (Moderate risk/moderate strength) and Profile 5 (Low stability, complex need/low strength) in comparison to the proportion of non-Indigenous men. This was found for Time 1, Time 2, and Time 3 (although at Time 3 a sixth profile emerged made up of a significantly higher proportion of Indigenous men, in comparison to the proportion of non-Indigenous men.

There were some notable gender similarities whereby the proportion of nonIndigenous men and women who were deemed low risk/low strength was greater than the proportion of Indigenous men and women, whereas there was a higher proportion of 
Indigenous men and women who had high scores on dynamic risk domains and complex needs than women and men who were non-Indigenous.

\section{Latent Transitional Probabilities for Women}

Time 1 to Time 2. For the transitional probabilities calculated between Time 1 and Time 2 (see Table 53), the majority of individuals remained in the same profile across timepoints (see Table 54 for profile counts and proportions from Time 1 to Time $2)^{22}$, with only about $4 \%$ of the sample $(n=70)$ switching from one profile to another.

Table 53

Transitional Probabilities between Profiles from Time 1 to Time 2 for Women

\begin{tabular}{cccc}
\hline & \multicolumn{3}{c}{ Profile at Time 2 } \\
\cline { 2 - 4 } Profile at Time 1 & 1 & 2 & 3 \\
\hline 1 & $\mathbf{. 9 5 3}$ & .018 & .029 \\
2 & .003 & .966 & .030 \\
3 & .018 & .039 & $\mathbf{. 9 4 3}$
\end{tabular}

Note. Profile 1 = Low risk/low strength, Profile $2=$ Gender-responsive, low risk/high strength, and Profile 3 = Aggressive, complex need/low strength.

${ }^{22}$ Fit indices indicated that the latent transition analysis was a very good fit to the data (Entropy $=.963$ ). 
Table 54

Profile Transitions for Women from Time 1 to Time 2

\begin{tabular}{lcc}
\hline \multirow{2}{*}{ Profile Transitions } & \multicolumn{2}{c}{ Women $(N=1,684)$} \\
\cline { 2 - 3 } & Count & $\%$ \\
\hline Profile 1 to Profile 1 & 770 & 45.72 \\
Profile 1 to Profile 2 & 15 & 0.89 \\
Profile 1 to Profile 3 & 19 & 1.13 \\
Profile 2 to Profile 1 & 2 & 0.12 \\
Profile 2 to Profile 2 & 523 & 31.06 \\
Profile 2 to Profile 3 & 17 & 1.01 \\
Profile 3 to Profile 1 & 4 & 0.24 \\
Profile 3 to Profile 2 & 13 & 0.77 \\
Profile 3 to Profile 3 & 321 & 19.06 \\
\hline
\end{tabular}

Note. The transitions that are bold represent those individuals who remained in the same profile from Time 1 to Time 2 (indicating no change in profile membership). Profile 1 = Low risk/low strength, Profile $2=$ Gender-responsive, low risk/high strength, and Profile $3=$ Aggressive, complex need/low strength.

Time 2 to Time 3. For the transitional probabilities calculated between Time 2 and Time 3 (see Table 55), the majority of individuals remained in the same profile across timepoints (see Table 56 for profile counts and proportions from Time 2 to Time $3)^{23}$, with only about $1.5 \%$ of the sample $(n=25)$ switching from one profile to a different profile.

\footnotetext{
${ }^{23} \mathrm{Fit}$ indices indicated that the latent transition analysis was a very good fit to the data (Entropy $=.975$ ).
} 
Table 55

Transitional Probabilities between Profiles from Time 2 to Time 3 for Women

\begin{tabular}{cccc}
\hline \multirow{2}{*}{ Profile at Time 2 } & \multicolumn{3}{c}{ Profile at Time 3 } \\
\cline { 2 - 4 } & 1 & 2 & 3 \\
\hline 1 & $\mathbf{. 9 9 0}$ & .006 & .004 \\
2 & .000 & .987 & .013 \\
3 & .012 & .029 & $\mathbf{. 9 5 9}$ \\
\hline
\end{tabular}

Note. Profile $1=$ Low risk/low strength, Profile $2=$ Gender-responsive, low risk/high strength, and Profile 3 = Aggressive, complex need/low strength.

Table 56

Profile Transitions for Women from Time 2 to Time 3

\begin{tabular}{lcc}
\hline \multirow{2}{*}{ Profile Transitions } & \multicolumn{2}{c}{ Women $(N=1,684)$} \\
\cline { 2 - 3 } Profile 1 to Profile 1 & Count & \% \\
Profile 1 to Profile 2 & 483 & 0.50 \\
Profile 1 to Profile 3 & 24 \\
Profile 2 to Profile 1 & 0 & 0.12 \\
Profile 2 to Profile 2 & 550 & 0 \\
Profile 2 to Profile 3 & 7 & 32.66 \\
Profile 3 to Profile 1 & 3 & 0.42 \\
Profile 3 to Profile 2 & 9 & 0.18 \\
Profile 3 to Profile 3 & 326 & 0.53 \\
\hline
\end{tabular}

Note. The transitions that are bold represent those individuals who remained in the same profile from Time 2 to Time 3 (indicating no change in profile membership). Profile $1=$ Low risk/low strength, Profile $2=$ Gender-responsive, low risk/high strength, and Profile 3 = Aggressive, complex need/low strength. 


\section{Latent Transitional Probabilities for Men}

For men, transitional probabilities could not be calculated between Time 2 and Time 3, as the interpretability of the results would not be meaningful, given that the number of profiles that emerged in Time 3 ( 6 profiles) was different from Time 2 ( 5 profiles). The transitions would not be indicative of true change as it is unclear whether individuals changed as a product of profile structure (e.g., 5 profiles versus 6 profiles), or because individuals actually changed from one profile to another. Nonetheless, transitional probabilities were examined between Time 1 and Time 2.

Time 1 to Time 2. For the transitional probabilities calculated between Time 1 and Time 2 (see Table 57) the majority of individuals remained in the same profile across timepoints (see Table 58 for profile counts and proportions from Time 1 to Time 2$)^{24}$. The largest amount of movement was from Profile 2 (at Time 1) to Profile 3 (at Time 2), although this represented only $1.17 \%$ of the sample $(n=78)$. Overall, only $5.6 \%$ of the sample switched to a different profile from Time 1 to Time $2(n=372)$.

\footnotetext{
${ }^{24} \mathrm{Fit}$ indices indicated that the latent transition analysis was a very good fit to the data (Entropy $=.966$ ).
} 
Table 57

Transitional Probabilities between Profiles from Time 1 to Time 2 for Men

\begin{tabular}{cccccc}
\hline Profile at & \multicolumn{5}{c}{ Profile at Time 2 } \\
\cline { 2 - 6 } Time 1 & 1 & 2 & 3 & 4 & 5 \\
\hline 1 & .971 & .002 & .010 & .013 & .004 \\
2 & .000 & .746 & .192 & .020 & .041 \\
3 & .000 & .009 & .946 & .030 & .015 \\
4 & .001 & .001 & .020 & .976 & .002 \\
5 & .000 & .018 & .130 & .020 & $\mathbf{. 8 3 1}$ \\
\hline
\end{tabular}

Note. Profile $1=$ Low risk/low strength, Profile $2=$ Aggressive, complex need/low strength, Profile 3 = Moderate risk/moderate strength, Profile $4=$ Low risk/high strength, and Profile $5=$ Low stability, complex need/low strength. 
Table 58

Profile Transitions from Time 1 to Time 2 for Men

\begin{tabular}{|c|c|c|}
\hline \multirow[b]{2}{*}{ Profile Transitions } & \multicolumn{2}{|c|}{$\operatorname{Men}(N=6,675)$} \\
\hline & Count & $\%$ \\
\hline Profile 1 to Profile 1 & 1,859 & 27.84 \\
\hline Profile 1 to Profile 2 & 3 & 0.05 \\
\hline Profile 1 to Profile 3 & 17 & 0.26 \\
\hline Profile 1 to Profile 4 & 24 & 0.36 \\
\hline Profile 1 to Profile 5 & 8 & 0.12 \\
\hline Profile 2 to Profile 1 & 0 & 0 \\
\hline Profile 2 to Profile 2 & 307 & 4.60 \\
\hline Profile 2 to Profile 3 & 78 & 1.17 \\
\hline Profile 2 to Profile 4 & 7 & 0.11 \\
\hline Profile 2 to Profile 5 & 16 & 0.24 \\
\hline Profile 3 to Profile 1 & 1 & 0.02 \\
\hline Profile 3 to Profile 2 & 20 & 0.30 \\
\hline Profile 3 to Profile 3 & 2,095 & 31.38 \\
\hline Profile 3 to Profile 4 & 64 & 0.96 \\
\hline Profile 3 to Profile 5 & 33 & 0.49 \\
\hline Profile 4 to Profile 1 & 2 & 0.03 \\
\hline Profile 4 to Profile 2 & 2 & 0.03 \\
\hline Profile 4 to Profile 3 & 32 & 0.48 \\
\hline Profile 4 to Profile 4 & 1,741 & 26.08 \\
\hline Profile 4 to Profile 5 & 3 & 0.05 \\
\hline Profile 5 to Profile 1 & 0 & 0 \\
\hline Profile 5 to Profile 2 & 6 & 0.09 \\
\hline Profile 5 to Profile 3 & 49 & 0.73 \\
\hline Profile 5 to Profile 4 & 7 & 0.11 \\
\hline Profile 5 to Profile 5 & 301 & 4.50 \\
\hline
\end{tabular}

Note. The transitions that are bold represent those individuals who remained in the same profile from Time 1 to Time 2 (indicating no change in profile membership). Profile $1=$ Low risk/low strength, Profile $2=$ Aggressive, complex need/low strength, Profile $3=$ Moderate risk/moderate strength, Profile $4=$ Low risk/high strength, and Profile $5=$ Low stability, complex need/low strength. 
Summary of latent transitions. Given that the same three profiles emerged over time for women, transitions between profiles could be assessed across all three timepoints. Results of the latent transitions indicated that for women, there was limited change between profiles from Time 1 to Time 2, and from Time 2 to Time 3 . Although limited, there was greater change in profiles between Time 1 and Time 2 in comparison to change in profiles between Time 2 and Time 3 (4\% vs. 1.5\%). For men, transitions between profiles could only be assessed between Time 1 and Time 2 due to a different profile structure at Time 3. Similar to the women, limited transitions among profiles occurred with only $5.6 \%$ of the men switching from one profile to another. Overall, for both men and women, there was limited change among profiles over time, and the change that did occur did not seem to follow a specific pattern.

\section{Typological Structure and Criminal Outcomes}

There are two ways to examine the relationship between distal outcomes (e.g., criminal outcomes) and latent transitions: (1) assesses how the distal outcome is related to change in profiles, or (2) how distal outcomes are related to the profile at the last timepoint of measurement (Perra, 2012). Transitional probabilities could not be examined across all timepoints due to differential profiles emerging at Time 3 for men. Additionally, there was limited amount of change between timepoints for women. As such, distal outcomes were examined in relation to profile membership at Time 3 (last measurement). As previously mentioned, the distal outcomes that were included are: technical violations, any new charges, violent charges, and any negative outcome. 
Women's profiles and criminal outcomes. The percentage of each profile of women that were charged of a new offence, violent offence, a technical violation, and any negative outcome are presented in Table 59.

Table 59

Proportions of Women who Committed Each Outcomes Across Profiles at Time 3

\begin{tabular}{lrrrrrrrr}
\hline & \multicolumn{2}{c}{ TV } & \multicolumn{2}{c}{ Any New } & \multicolumn{2}{c}{ Violent } & \multicolumn{2}{c}{ Negative outcome } \\
\cline { 2 - 8 } Profile & $\boldsymbol{n}$ & $\mathbf{\%}$ & $\boldsymbol{n}$ & $\mathbf{\%}$ & $\boldsymbol{n}$ & $\mathbf{\%}$ & $\boldsymbol{n}$ & $\%$ \\
\hline Profile 1 & 26 & 3.3 & 53 & 6.6 & 20 & 2.5 & 68 & 8.5 \\
Profile 2 & 26 & 4.5 & 39 & 6.8 & 11 & 1.9 & 52 & 9.1 \\
Profile 3 & 45 & 14.5 & 56 & 18.1 & 30 & 9.7 & 73 & 23.5 \\
\hline
\end{tabular}

Note. $\mathrm{TV}=$ Technical violations. Profile $1=$ Low risk/low strength $(n=801)$, Profile $2=$ Gender-responsive, low risk/high strength $(n=573)$, and Profile $3=$ Aggressive, complex need/low strength $(n=310)$.

Not surprisingly, Profile 3 (Aggressive, complex need/low strength) had the highest proportion of all four types of criminal outcomes. Profile 1 (Low risk/low strength) and Profile 2 (Gender-responsive, low risk/high strength) reported similar proportions of each type of criminal outcome, which is also demonstrated in Table 60 where there were no significant differences between Profile 1 (Low risk/low strength) and Profile 2 (Gender-responsive, low risk/high strength). 
Table 60

Profile Comparisons of Various Criminal Outcomes for Women

\begin{tabular}{lcc}
\hline Technical violation & Odds Ratio & $\boldsymbol{p}$ \\
\hline Profile 1 to Profile 2 & 0.737 & .227 \\
Profile 1 to Profile 3 & 0.220 & $<.001$ \\
Profile 2 to Profile 3 & 0.298 & $<.001$ \\
\hline Any new charge & Odds Ratio & $\boldsymbol{p}$ \\
\hline Profile 1 to Profile 2 & 1.025 & .915 \\
Profile 1 to Profile 3 & 0.345 & $<.001$ \\
Profile 2 to Profile 3 & 0.337 & $<.001$ \\
\hline Any negative outcome & Odds Ratio & $\boldsymbol{p}$ \\
\hline Profile 1 to Profile 2 & 0.967 & .868 \\
Profile 1 to Profile 3 & .0325 & $<.001$ \\
Profile 2 to Profile 3 & 0.336 & $<.001$ \\
\hline New violent charge & Odds Ratio & $\boldsymbol{p}$ \\
\hline Profile 1 to Profile 2 & 1.291 & .562 \\
Profile 1 to Profile 3 & 0.259 & $<.001$ \\
Profile 2 to Profile 3 & .0200 & $<.001$ \\
\hline Note. Profile $1=$ Low isk/low & & \\
\hline
\end{tabular}

Note. Profile $1=$ Low risk/low strength $(n=801)$, Profile $2=$ Gender-responsive, low risk/high strength $(n=573)$, and Profile $3=$ Aggressive, complex need/low strength $(n=$ $310)$.

Men's profiles and criminal outcomes. Distal outcomes were examined in relation to profiles at Time 3 (last measurement). The distal outcomes included were technical violations, any new charges, violent charges, and any negative outcome. The proportions of each profile that experienced the various criminal outcomes are presented in Table 61. 
Table 61

Proportions of Men who Committed Each Criminal Outcome Across Profiles at Time 3

\begin{tabular}{lcrcrcrcr}
\hline & \multicolumn{2}{c}{ TV } & \multicolumn{2}{c}{ Any New } & \multicolumn{2}{c}{ Violent } & \multicolumn{2}{c}{ Negative outcome } \\
\cline { 2 - 8 } Profiles & $\boldsymbol{n}$ & \% & $\boldsymbol{n}$ & $\%$ & $\boldsymbol{n}$ & $\%$ & $\boldsymbol{n}$ & $\%$ \\
\hline Profile 1 & 78 & 3.9 & 157 & 7.9 & 86 & 4.4 & 181 & 9.2 \\
Profile 2 & 38 & 14.2 & 43 & 16.0 & 29 & 10.8 & 56 & 20.9 \\
Profile 3 & 120 & 5.3 & 240 & 10.7 & 143 & 6.4 & 284 & 12.6 \\
Profile 4 & 29 & 3.3 & 56 & 6.4 & 22 & 2.5 & 70 & 8.0 \\
Profile 5 & 34 & 13.1 & 45 & 17.3 & 28 & 10.8 & 58 & 22.3 \\
Profile 6 & 121 & 11.5 & 176 & 16.8 & 104 & 9.9 & 219 & 20.9 \\
\hline
\end{tabular}

Note. $\mathrm{TV}=$ Technical violations. Profile $1=$ Low risk/low strength $(n=1,977)$, Profile 2 $=$ Aggressive, complex need/low strength $(n=268)$, Profile $3=$ Moderate risk/moderate strength $(n=2,246)$, Profile $4=$ Low risk/high strength $(n=875)$, Profile $5=$ Low stability, complex need/low strength $(n=260)$, and Profile $6=$ Moderate complex need/low strength $(n=1,049)$.

Across all four criminal outcomes, Profile 1 (Low risk/low strength) and Profile 4 (Low risk/high strength) did not significantly differ in terms of proportions of technical violations, any new charges, violent charges, and any negative outcome. Similarly, Profile 2 (Aggressive, complex need/low strength) did not significantly differ from Profile 5 (Low stability, complex need/low strength) and Profile 6 (Moderate complex need/low strength) in terms of proportions of men who committed each of the four criminal outcomes. Profile 5 (Low stability, complex need/low strength) did not differ from Profile 6 (Moderate complex need/low strength) in terms of proportions of technical violations, new charges, any negative outcome, and violent charges. Finally, Profile 3 (Moderate risk/moderate strength) did not differ from Profile 4 (Low risk/high strength) in terms of proportions of those who committed a technical violation, but did differ across all other outcomes (see Table 62). 
Table 62

Profile Comparisons of Various Criminal Outcomes for Men

\begin{tabular}{|c|c|c|c|c|c|c|c|c|}
\hline \multirow{2}{*}{ Profile comparisons } & \multicolumn{2}{|c|}{ Technical violation } & \multicolumn{2}{|c|}{ Any new charge } & \multicolumn{2}{|c|}{ Violent charge } & \multicolumn{2}{|c|}{ Negative outcome } \\
\hline & OR & $p$ & OR & $p$ & OR & $p$ & OR & $p$ \\
\hline Profile 1 to Profile 2 & 0.249 & $<.001$ & 0.453 & $<.001$ & 0.381 & $<.001$ & 0.383 & $<.001$ \\
\hline Profile 1 to Profile 3 & 0.719 & .013 & 0.723 & .001 & 0.679 & .001 & 0.701 & $<.001$ \\
\hline Profile 1 to Profile 4 & 1.117 & .646 & 1.250 & .236 & 1.844 & .078 & 1.139 & .426 \\
\hline Profile 1 to Profile 5 & 0.271 & $<.001$ & 0.426 & $<.001$ & 0.387 & $<.001$ & 0.360 & $<.001$ \\
\hline Profile 1 to Profile 6 & 0.335 & $<.001$ & 0.453 & $<.001$ & 0.443 & $<.001$ & 0.406 & $<.001$ \\
\hline Profile 2 to Profile 3 & 2.891 & .001 & 1.597 & .042 & 1.781 & .046 & 1.830 & .007 \\
\hline Profile 2 to Profile 4 & 4.492 & .003 & 2.760 & .004 & 4.837 & .010 & 2.973 & .001 \\
\hline Profile 2 to Profile 5 & 1.091 & .747 & 0.940 & .789 & 1.016 & .955 & 0.939 & .766 \\
\hline Profile 2 to Profile 6 & 1.347 & .211 & 1.001 & .997 & 1.162 & .542 & 1.060 & .745 \\
\hline Profile 3 to Profile 4 & 1.554 & .116 & 1.728 & .012 & 2.716 & .014 & 1.624 & .012 \\
\hline Profile 3 to Profile 5 & 0.377 & $<.001$ & 0.588 & $<.001$ & 0.571 & .001 & 0.513 & $<.001$ \\
\hline Profile 3 to Profile 6 & 0.466 & $<.001$ & 0.626 & $<.001$ & 0.653 & $<.001$ & 0.579 & $<.001$ \\
\hline Profile 4 to Profile 5 & 0.243 & $<.001$ & 0.340 & $<.001$ & 0.210 & $<.001$ & 0.316 & $<.001$ \\
\hline Profile 4 to Profile 6 & 0.300 & $<.001$ & 0.363 & $<.001$ & 0.240 & $<.001$ & 0.356 & $<.001$ \\
\hline Profile 5 to Profile 6 & 1.235 & .377 & 1.065 & .753 & 1.144 & .596 & 1.128 & .518 \\
\hline
\end{tabular}

Note. OR $=$ Odds Ratio. Profile $1=$ Low risk/low strength, Profile $2=$ Aggressive, complex need/low strength, Profile $3=$ Moderate risk/moderate strength, Profile $4=$ Low risk/high strength, Profile $5=$ Low stability, complex need/low strength, and Profile $6=$ Moderate complex need/low strength. 
Summary of profile structure and criminal outcome. Results indicated that significantly more women in Profile 3 (Aggressive, complex need/low strength) had a technical violation, any new charge, new violent charge, and any negative outcome than women in the other two profiles. However, there were no significant differences in criminal outcomes between Profile 1 (Low risk/low strength) and Profile 2 (Genderresponsive, low risk/high strength). In contrast, for men there were three profiles that committed the majority of the various criminal outcomes. These profiles were: Profile 2 (Aggressive, complex need/low strength) Profile 5 (Low stability, complex need/low strength) and Profile 6 (Moderate complex need/low strength). Among both men and women, the profiles with the highest reoffence rates were those with complex needs, aggression, and low strengths.

\section{Supplementary Analysis}

A supplemental analysis was conducted whereby an LPA was ran for those who had an initial full SPIn assessment within 90 days of start of supervision and who had recidivism information, resulting in 16,605 men and 3,895 women. This supplementary analysis was conducted to examine whether including those who recidivated prior to the three assessment timepoints (i.e., prior to 14-months post initial assessment), altered the profile structure that emerged for men and women independently. The profiles that emerged were also examined in relation to the various criminal outcomes using a 3-year fixed follow-up. These supplementary analysis results are presented in Appendix K.

Overall, results of the supplemental LPA for women indicated that a 3-profile structure fit the data best. The three profiles that emerged were: Profile $1=$ Aggressive, complex need/low strength, Profile 2 = Moderate complex need/moderate strength, and 
Profile 3 = Low risk/high strength. The largest proportion of women made up the Moderate complex need/moderate strength profile (50.8\%) and the smallest proportion of women made up the aggressive, complex need/low strength profile (10.81\%).

In contrast, for men, a 5-profile structure fit the data best. The five profiles that emerged were: Profile 1 = Aggressive, moderate complex need/low strength, Profile $2=$ Low stability, complex need/low strength, Profile 3 = Aggressive, high complex need/low strength, Profile $4=$ Moderate risk/moderate strength, and Profile $5=$ Low risk/high strength. The largest proportion of men made up the Moderate risk/moderate strength profile $(48.62 \%)$, which was a similar finding to the women. The smallest proportion of men made up the Aggressive, high complex need/low strength profile (2.81\%), with the next smallest proportion of men making up the Aggressive, moderate complex need/low strength profile (7.61\%). Thus, for both the men and women, about $10 \%$ of the samples were comprised of individuals who were aggressive with complex needs and scoring low on strengths. In addition, both genders had a Low risk/high strength profile, however, while $28.18 \%$ of men fell into this profile, $38.38 \%$ of women made up this profile. Finally, for both men and women, those who are aggressive with complex needs and low strengths demonstrated the highest rates of criminal behaviour for all four offending outcomes (any new charge, any violent charge, technical violations, and any negative outcome). 


\section{Study 2 Discussion}

The current study was the first to incorporate gender-neutral and genderresponsive dynamic risks, needs, and strengths to assess offender typologies of men and women on community supervision, and examine how these typologies change over time. Unlike previous typological research (e.g., Piquero, 2008; Salisbury \& Van Voorhis, 2009), this study integrated tenets from three separate, but complementary criminogenic theories, the pathways perspective, mainstream gender-neutral correctional perspective (e.g., RNR), and a strengths-based perspective. Furthermore, the majority of research either focuses solely on males (e.g., Simourd et al., 1994), females (e.g., Dehart, 2018), or youth (e.g., Brown et al., 2020), and very few studies have examined how typological membership changes over time, with the exception of Greiner (2015). The main goal of the current study was to examine whether or not there are gender differences in the typologies of offenders that emerged for men and women. A secondary goal was to examine the relationship between offender typologies and reoffending outcomes, such as technical violations, new charges, violent charges, and any negative outcome. This research was conducted to help inform why individuals engage in crime, assist with treatment and rehabilitation of justice-involved men and women, help guide case management practices, and help determine who are more likely to reoffend (Jones \& Harris, 1999).

\section{Offender Typologies}

The first research question examined whether typologies can be delineated among justice-involved men and women. Based on previous research, it was hypothesized that between four and six typologies would emerge for both genders; however, no hypotheses 
were made regarding the types that would emerge. The results demonstrated that women on community supervision can be classified into three distinct offender typologies based on treatment needs and strengths that are gender-informed. The following profiles emerged at each timepoint: 1) Low risk/low strength profile scoring low across all domains; 2) Gender-responsive, low risk/high strength profile scoring low across all dynamic risk domains, with the exception of mental health and childhood adversity needs, but highest on all strength domains; 3) Aggressive, complex need/low strength profile scoring high on aggression and violence, as well as all dynamic risk domains and gender-responsive needs, but scored low on strength domains.

In contrast, men on community supervision can be classified into five distinct offender typologies based on treatment needs and strengths that are gender-informed. The following profiles emerged at each timepoint: 1) Low risk/low strength profile scoring low across all domains; 2) Aggressive, complex need/low strength profile scoring high on aggression and violence, as well as all dynamic risk domains and gender-responsive needs, but low on strength domains; 3) Moderate risk/moderate strength profile scoring moderate on all domains; 4) Low risk/high strength profile scoring low on all risk domains and gender-responsive needs, but highest on all strength domains; and 5) Low stability, complex need/low strength profile scoring high on dynamic risk domains, especially employment and stability, as well as gender-responsive needs, but low on aggression and strength domains.

It is interesting that for both men and women two distinct types of low risk profiles emerged, one scoring low on strengths and one scoring high on strengths. This may have implications on the treatment and classification of low risk offenders. For 
instance, perhaps there are additional categories of justice-involved men and women other than low, moderate or high risk that need to be considered when determining frequency of contact with community supervision officers, program placements, and even probation conditions. In addition, having two "types" of low risk justice-involved individuals can have implications for how these individuals are managed in the community. For example, some jurisdictions implement a low-intensity supervision model whereby those rated lowest risk report monthly to a computerized kiosk, rather than to a community supervision officer, to answer questions regarding their contact details, employment, and any problems they may be facing (Barnes et al., 2010). This approach allows for more resources to be placed towards higher risk cases and has been found to be successful in reducing the rate of re-arrests over a two-year period (Wilson, Naro, \& Austin, 2007). Given that the current sample was predominately low-risk justiceinvolved men and women, it is important to examine whether moderate and high risk samples also display similar patterns in terms of strengths - as this could be a potential direction for the use of overrides in a variety of contexts (i.e., security classification, programming needs, and frequency of contact with supervision officers).

\section{Stability of Offender Typologies}

The second research question examined whether the same typologies of women and men that emerged at Time 1 also emerged at Time 2 and Time 3. Findings indicate that for the women, the same three profiles emerged across all timepoints. For men, however, the same five profiles emerged at Time 1 and Time 2, but at Time 3, a sixth profile-structure emerged: a moderate complex need/low strength profile. 
For women, the three profiles maintained similar means for all dynamic risk, gender-responsive needs, and strength domains over the three timepoints. For men, however, from Time 1 to Time 2, the mean scores on all domains remained consistent for each profile, with slight increases in attitude strength scores and slight decreases in stability strength and substance use risk scores across each profile. Although decreases in substance use and increases in prosocial attitudes were expected as successful time in the community increase, it was not expected that stability strength scores would decrease. At Time 3, the low risk/low strength profile and the aggressive, complex need/low strength profile had similar mean scores across all domains as what was seen at Time 1 and Time 2. The moderate risk/moderate strength profile, the low risk/high strength profile, and the low stability, complex need/low strength (profiles 3 to 5), while demonstrating similar trends in scores on each domain, had different mean scores on the majority of risk, need, and strength domains. However, given that an additional profile emerged at the third timepoint, the change in domain scores in the profiles that were similar across timepoints (Profiles 1 to 5) are most likely due to the different typological structure, rather than due to men's change in scores (especially considering the discrepancy in sample sizes among the profiles from Time 1 to Time 3; for example Profile 4 at Time $1[n=1,718]$ and at Time $2[n=1,843]$ was much larger than the sample size at Time $3[n=875])$.

\section{Gender and Typological Membership}

The third research question pertains to whether typology membership differs based on gender. Previous pathways research has indicated that there are gendered subtypes of justice-involved individuals that emerge (e.g., Brennan et al., 2012; Dehart, 2018; Salisbury \& Van Voorhis, 2009). Much of this evidence, however, has stemmed 
from studies that did not include a male comparison group, limiting the ability to conclude that these subtypes are truly unique for females. Previous typological research that has included a male comparison group has found support for typologies that are not mutually exclusive for males and females (e.g., Daly, 1994; Greiner, 2015; Jones et al., 2014), although most of this research has been conducted on youth. Nonetheless, it was hypothesized that there would be similar typologies that emerge for both men and women and that at least one typology would emerge comprised predominately of genderresponsive need and strength factors for both genders.

The results indicated some gender differences and some similarities. As hypothesized, similar profiles emerged for the men and the women. For instance, two of the three profiles that emerged for the women also emerged for the men (the low risk/low strength profile, and the aggressive, complex need/low strength profile). The third profile for women that emerged resembled a gender-responsive profile (scoring low on genderneutral risk factors but higher on more gender-responsive factors such as mental health, childhood adversity, and substance use). Although for the men no profiles emerged comprised predominately of gender-responsive factors, there were two profiles that emerged that included a mixture of gender-neutral and gender-responsive needs (deemed complex needs when both were present). These results are similar to those found by previous youth typological studies. For instance, one study conducted by Jones et al. (2014) used a sample of 663 justice-involved girls and 1,175 justice-involved boys on community supervision in New York State who were assessed using the YASI (Orbis Partners, 2000). Using multidimensional scaling, they found that for girls, almost half followed a gender-responsive pathway defined by gender-responsive factors such as 
abuse, poverty, and mental health issues. In contrast, while the boys did not exhibit a gender-responsive pathway, about a quarter of the sample did exhibit a mixed pathway which was defined by both gender-neutral and gender-responsive factors such as antisocial attitudes, impulsivity, abuse, and mental health issues.

Taken together, these results indicate that although gender-responsive items are important to consider for girls and women, there is a portion of boys and men who demonstrate gender-responsive needs and who would benefit from having these areas assessed and targeted. Further, while previous research has continuously indicated that gender-neutral risk domains are relevant for women (e.g., Andrews et al., 2012; Green \& Campbell, 2006), there were no profiles made-up predominately of gender-neutral domains that emerged for women. As such, these findings denote the utility of incorporating gender-informed approaches; that is, combined gender-neutral and genderresponsive perspectives, to assessing risk, need and strengths among both justiceinvolved men and women.

Another interesting finding was that for both men and women, those who were deemed aggressive, had a mixture of gender-neutral and gender-responsive needs (deemed complex needs), and low strengths. However, it is important to keep in mind that just because similar typologies emerged for men and women, they do not represent the same make-up in terms of mean scores across the domains. What is considered the aggressive profile for women, is relative to that entire sample of women. And what is considered the aggressive profile for men, is relative to the entire sample of men. Similarly, profiles deemed high strength have scores that are higher than the rest of the sample, but do not necessarily coincide with SPIn score classifications of high, moderate, 
and low scores. As such, while similar profile trends have emerged among men and women, the scores making up these profiles are quite different. In terms of the aggressive, complex need//low strength profile that emerged for both genders, the mean aggression risk score for women at Time 1 was only 1.02 (which was much higher than the rest of the women), in comparison to the mean aggression score for men at Time 1, which was 3.87. As such, while the trends can be compared, the actual mean scores on the domains making up the profiles can not be. Although direct comparisons between genders are cautioned, men and women had similar profile trends that emerged, consisting of gender-responsive, gender-neutral, and strength domains, suggesting that men and women may be more similar than different in their treatment profiles.

\section{Stability of Typological Membership}

The fourth research question examined the extent to which the proportions of individuals belonging to each typology remain stable across timepoints (or whether the proportions of individuals belonging to each typology changed over time). Given that the same three profiles emerged over time for women, transitions between profiles could be assessed across all three timepoints. Results of the latent transitions indicated that for women, there was limited change between profiles from Time 1 to Time 2 and from Time 2 to Time 3. Although limited, there was greater change in profiles between Time 1 and Time 2 in comparison to change in profiles between Time 2 and Time 3 (4\% vs. 1.5\%).

For men, transitions between profiles could only be assessed between Time 1 and Time 2 due to a different profile structure at Time 3 . This is because changes in profiles from Time 2 to Time 3 may not actually be due to true offender change, but rather due to profile structure changes. Nonetheless, latent transitions were examined between Time 1 
and Time 2, and similar to the women, limited transitions between profiles occurred with only $5.6 \%$ of the men switching from one profile to another.

Overall, for both men and women, there was limited change between profiles over time, and the change that did occur did not seem to follow a specific pattern. There are several plausible reasons for this. First, the sample included in the current analyses are mainly low risk (55.1\% men and 62.5\% women based on SPIn overall risk score) and individuals who reoffended within 9 to 14-months of the initial assessment (based on when the last SPIn assessment occurred) were excluded. This allowed for three timepoints, which is required to accurately assess change over time (Babchishin, 2013; Brown et al., 2009; Lloyd, 2015). As such, because the sample is comprised mainly of low risk offenders, there is less room for change in dynamic risk and strength domains. Since two profiles of men and women are comprised of low dynamic risk domain scores, the likelihood of moving to another profile with higher dynamic risk domain scores is low.

Second, change in dynamic need and strength domains may require more time. For men, transitions were assessed only between Time 1 and Time 2 (due to similar profile structures at both these timepoints), there are only a matter of months between these two timepoints. In particular, Time 2 SPIn assessments occur between 3 and 8 months post initial assessment (Time 1) and as such, may not be enough time to exhibit changes in the various dynamic risk and strength domain scores.

Although the SPIn is comprised of a number of dynamic items, which are combined to create dynamic domains, it is important to consider that not all dynamic items change at the same rate. The extent to which the items making up the dynamic 
domains are stable dynamic versus acute dynamic in nature is unknown. Research has found that dynamic items can be divided into two categories: stable dynamic — which refers to factors that are more long-standing that change over a matter of months or years, and acute dynamic — which refers to factors that change more rapidly over time, such as days or weeks (Hanson et al., 2007). For example, items assessing attitudes may be more stable and thus, change may be quite gradual, whereas other items assessing substance use may be more acute. Notably, one assessment tool similar to the SPIn, in that it measures a variety of dynamic risk and strength factors, is the Dynamic Risk Assessment of Offender Re-entry (DRAOR; Serin, 2007), which has specific domains that are comprised solely of either acute factors or stable factors, recognizing that dynamic items change at different rates. As such, the gradual rate of change exhibited in the current study may potentially be due to the number of items that are stable dynamic relative to the number of items that are acute dynamic. Research on the SPIn is needed at the item level to examine rate of change among specific dynamic items to better understand the dynamic nature of the domain scores.

\section{Typological Membership and Criminal Outcomes}

One of the main reasons for identifying offender typologies is to determine if there are certain groups of justice-involved men and women that are more likely to reoffend (Jones \& Harris, 1999). By determining if there are certain profiles that are more likely to reoffend then others, treatment and rehabilitation efforts can be tailored to target the domains most pertinent to those offenders, and better inform supervision efforts (e.g., frequency of contact). As such, the final research question examined whether there were certain profiles of offenders that were more likely to reoffend than other profiles. 
Results indicated that significantly more women in Profile 3 (Aggressive, complex need/low strength) had technical violations, new charges, new violent charges, and any negative outcome than women in the other two profiles. However, there were no significant differences in criminal outcomes between Profile 1 (Low risk/low strength) and Profile 2 (Gender-responsive, low risk/high strength). Strengths did not seem to influence reoffending rates, whereby those who were low risk/low strength and those who were low risk/high strength had similar rates of all four outcomes — although those with high strengths did have slightly lower rates of violent charges, but this difference was not significant.

In contrast, for men there were three profiles that had the highest rates of the various criminal outcomes: Profile 2 (Aggressive, complex need/low strength) Profile 5 (Low stability, complex need/low strength) and Profile 6 (Moderate complex need/low strength). These three profiles had significantly higher technical violations, violent charges, any new charges, and any negative outcome than Profile 1 (Low risk/low strength), Profile 3 (Moderate risk/moderate strength), and Profile 4 (Low risk/high strength). An interesting finding that emerged for the men was that those who were nonaggressive had similar rates of reoffending to those who were aggressive if they scored high on complex needs (both gender-responsive needs and gender-neutral dynamic risk domains). That is, men who have complex needs tend to be more likely to reoffend than men who score moderately on gender-neutral risk factors alone (e.g., moderate risk/moderate strength).

In sum, and not surprisingly, the highest rate of reoffending across all outcomes was for men and women characterized as aggressive, complex need/low strength. 
Conversely, all reoffending outcomes were lowest for both men and women who were characterized as scoring low on dynamic risk domains, regardless of scores on strengths. This was the low risk/low strength profile and the low risk/high strength profile for men and the low risk/low strength profile and the gender-responsive, low risk/high strength profile for women.

\section{Indigenous Men and Women and Profile Membership}

Given the high proportion of Indigenous men $(n=867 ; 13.0 \%)$ and women $(n=$ $403 ; 23.9 \%$ ) in the current sample, Indigenous status was assessed as an auxiliary variable. Overall, there were significant differences in the proportion of Indigenous and proportion of non-Indigenous men and women making up the majority of profiles. Results found that more Indigenous than non-Indigenous women were classified as having complex needs - indicating both gender-neutral and gender-responsive needs. These findings for women are not surprising given that past research has found that women have a whole host of complex needs, including high rates of childhood abuse, witnessing violence, mental health concerns, and substance use needs (Brown, Barker, et al., 2018; Stewart et al., 2017). In fact, Indigenous women have needs that are arguably unique and more complex compared to non-Indigenous women (Gutierrez \& Wanamaker, 2020). For instance, in addition to these gender-responsive needs, other needs may stem from residential school experience, intergenerational trauma, child welfare involvement, family history of suicide, and extreme poverty and poor living conditions on reservations (Office of the Correctional Investigator, 2015). Unfortunately, these culturally-relevant factors were not considered in typological profile development, and thus the results focusing on Indigenous status should be interpreted with caution. 
For the men, different trends were found. Surprisingly, a larger proportion of Indigenous men make up profiles characterized by more strengths (e.g., moderate risk/moderate strength, and low risk, high strength profiles). However, the reasons behind this result is unclear and require further investigation.

Overall, these findings highlight the fact that, while there are gender differences, there are also differences between Indigenous and non-Indigenous men, and Indigenous and non-Indigenous women. As such, while it is a good starting point, it is not enough to solely examine gender differences in men and women without considering other intersecting factors such as ethnicity or class. Unfortunately, few studies have incorporated the intersection of gender and Indigenous status, specifically (Bartels, 2012). As such, additional research is needed looking at different typologies among different ethnicities, for both men and women that include culturally-relevant factors. Identifying whether unique typologies of offenders emerge among justice-involved Indigenous men and women, incorporating culturally-relevant risk and need factors in addition to gender-neutral, gender-responsive, and strengths-based factors, can assist with tailoring treatment to target appropriate need domains. A more fulsome discussion of intersectionality can be found in the Study 1 discussion.

\section{Limitations and Directions for Future Research}

Assessment selection. The biggest limitation pertains to the large amount of data that was lost. Specifically, anyone with less than three assessments were removed, and any SPIn assessments conducted after the 14 months post initial assessment were also removed. To adequately assess change in profile structures over time, three timepoints were required for each man and woman in the sample. The assessment schedule was 
grouped into the following three timepoints: Time $1=$ initial assessment occurring within 90 days of supervision start, Time $2=3$ to 8 months post initial assessment, and Time $3=$ 9 to 14 months post initial assessment. If there was more than one assessment during that time period, one of the assessments was randomly selected to represent that point in time. Selecting these specific time periods allowed for the largest sample inclusion.

Additionally, ensuring that there were three assessment periods resulted in losing any cases who recidivated within the 14-month time period, limiting the sample to those who are predominately low risk. Thus, typologies are not generalizable to all justiceinvolved men and women on community supervision, but rather to those who are successful in the community. However, it is important to note that upon examining the average risk level for the 28,423 men and women with full assessment information from the larger archival SPIn dataset for which this data is subsumed, the majority are considered low risk $(53.5 \%)$ rather than moderate $(37.5 \%)$ or high risk $(9.0 \%)$. Further, research conducted by Bourgon et al. (2018) have concluded that the typical offender in Alberta is considered to be low risk; that is, the median risk score on the SPIn in Alberta corresponds to a classification of low risk. As such, the typological findings from the current study may in fact be quite similar to the population of men and women on community supervision in Alberta, however, further investigation is warranted.

Supplemental LPA. To account for the significant loss of data, a supplemental LPA was conducted on those from the initial release cohort - that is, anyone who had a complete SPIn Full Assessment within 90 days of start of supervision. This was conducted to see how the typological structure changes when including men and women with less than three assessments and who recidivate prior to 14 months (see Appendix K). 
Overall, results indicated that similar profiles emerged for the full sample as those in the main analyses. Three profiles emerged for the women and five profiles emerged for the men, indicating that offender typologies can be meaningfully delineated among the larger sample of justice-involved men and women on community supervision in Alberta. For the women, three profiles emerged: Profile $1=$ Aggressive, complex need/low strength, Profile $2=$ Moderate complex need/moderate strength, and Profile $3=$ Low risk/high strength. In comparison, the five profiles that emerged for the men were: Profile 1 = Aggressive, moderate complex need/low strength, Profile $2=$ Low stability, complex need/low strength, Profile 3 = Aggressive, high complex need/low strength, Profile $4=$ Moderate risk/moderate strength, and Profile $5=$ Low risk/high strength.

As expected, the rates of reoffending were substantially higher among the supplemental sample as those who reoffended within 14 months were included in analyses unlike the main LPA/LTA. In terms of reoffending outcomes, both men and women who were aggressive had more new charges, violent charges, technical violations, and any negative outcome. Interestingly, these rates were not significantly different from those who were non-aggressive but had complex needs - a combination of gender-neutral and gender-responsive needs. In fact, for men, in comparison to those who were considered aggressive with complex needs and low strengths, those who were nonaggressive but had moderate complex needs and low strengths had very similar reoffending rates across all outcomes, with the exception of violent charges.

These results had some similarities and differences to the findings of the main analyses for both genders. In both the main analyses and supplemental LPA for women, an aggressive, complex need/low strength profile and a low risk/high strength profile 
emerged. However, for the supplemental analyses, the third profile was made up of moderate risk/moderate strength women whereas the main analyses had a low risk/low strength profile. This is not surprising given that the main analyses were made up of predominately more low risk women. In contrast, for the men, the typological structure that emerged in both the main analyses and the supplemental analyses were very similar, with one exception - in the supplemental LPA there were two profiles characterized as aggressive, whereas in the main analyses there was only one. Again, given the main analyses were comprised predominately of low risk men, these results are not surprising.

Finally, in terms of criminal outcomes, it was not surprising that for women, Profile 1 (Aggressive, complex need/low strength) displayed the highest rate of all four criminal outcomes - technical violations, any new charges, violent charges, and any negative outcome. For men, Profile 2 (Low stability, complex need/low strength) and Profile 3 (Aggressive, high complex need/low strength) had the highest rate of any new charges, technical violations, and any negative outcome. For violent charges, however, the aggressive, high complex need/low strength profile scored significantly higher than any other profile. These results are surprising because there are two aggressive profiles that emerged; however, the aggressive profile with high complex needs was much more likely to offend than those who are aggressive and have moderate complex needs. Additionally, those who belong to the low stability, complex need/low strength profile (who are non-aggressive) tend to have more charges and technical violations than the aggressive, moderate complex need/low strength profile. This indicates that complex needs and stability (e.g., employment, place to live) may play a larger role in men's immediate reoffending and may in fact be more (or just as) important as aggression. As 
such, more research on the effects of complex needs and reoffending following the first year on supervision is warranted.

How gender-informed is the SPIn? Another limitation pertains to the nature of the dynamic risks and strengths included in the analyses. First, although the SPIn is considered a gender-informed risk assessment tool which captures both genderresponsive and gender-neutral needs and strengths (Jones \& Robinson, 2017b), there may be additional gender-responsive needs that are not captured within a dynamic risk or strength domain. For example, gender-responsive researchers have highlighted the following gender-responsive needs: self-esteem, self-efficacy, parental stress, victimization and abuse, relationship dysfunction, mental health concerns (especially depression), and poverty and homelessness (Belknap, 2015; Van Voorhis et al., 2010). Upon inspection, the majority of these gender-responsive items are captured within the SPIn Full Assessment, with the exception of self-esteem and self-efficacy.

Arguably, while the SPIn is considered a gender-informed tool, gender-responsive researchers have questioned the utility of gender-informed assessments (Salisbury, Boppre, \& Kelly, 2016). Specifically, while incorporating a combination of genderneutral and gender-responsive items have demonstrated improved predictive accuracy of reoffending outcomes (Salisbury et al., 2016; Van Voorhis et al., 2010), genderresponsive scholars argue that women remain an after thought. These gender-informed tools are thought to simply be amended gender-neutral tools whereby few genderresponsive items are added which are operationalized or captured in a traditional manner (Salisbury et at., 2016; Van Voorhis et al., 2010). Despite these arguments in support of gender-responsivity, there has been a substantial amount of research highlighting the 
predictive utility of gender-neutral risk factors for justice-involved women (e.g., Dowden \& Andrews, 1999; Scanlan et al., 2020). Thus, including both gender-neutral and genderresponsive items can ensure a holistic approach to risk assessment practices. Nonetheless, additional research is needed focusing on the role of gender-responsive needs with justice-involved women (and men).

Item level limitation. Another limitation was the inability to look at item-level data. The current study utilized domain level information, each comprised of several items, to inform treatment typologies. Thus, the influence of any specific item was masked by the combined domain total score. Additionally, many of the domains had gender-responsive items embedded within, making it difficult to determine the nature of the items that were influencing domain scores and thus the treatment profiles. For example, while gender-responsive researchers have highlighted the importance of childhood abuse and maltreatment on women's involvement in crime (e.g., Belknap, 2015; Van Voorhis et al., 2010), the current study included a measure of adverse childhood experiences, but could not speak specifically to childhood abuse. Moreover, the items included in the ACE score were also included within the other domain scores, which leads to some overlap in items. That is, it was not possible to extract the items that were included to create the ACE score from the various domains. Future research should examine SPIn item-level data to see if there are specific items that cluster together (rather than at the domain level) and that are most predictive of reoffending outcomes, for both men and women.

Additional limitations and directions for future research that are applicable to this study are presented in Study 1, including the definition of gender, as well as SPIn-related 
concerns such as ability to assess change, assessment factor structure, item measurement, and lack of psychometric properties.

\section{Summary}

The current study was the first to incorporate gender-neutral and genderresponsive dynamic risks and strengths to inform treatment typologies among a large sample of justice-involved men and women on community supervision. Using a personcentered approach across multiple timepoints, the typological structure of justiceinvolved men and women were assessed over time. In sum, results indicated that there were many similarities between men and women in terms of the profiles that emerged. First, for both genders, those who were low risk were split into two profiles — one with high strengths and one with low strengths. Second, both men and women had profiles comprised of complex needs; that is, both gender-neutral risks and gender-responsive needs. Third, for both genders there was an aggressive profile that emerged which also demonstrated complex needs and this profile of men and women were more likely to receive a technical violation, or new charge, violent charge, and/or any negative outcome. Finally, there was limited change among typologies over time, for both genders.

Notably, there were also some gender differences. First, the women had one profile that emerged with predominately gender-responsive needs (with few genderneutral dynamic risk factors present). Second, while three profiles emerged over time for women, five profiles emerged for men. Finally, the heterogeneity of typological structure increased for men, whereby a sixth profile emerged at the third timepoint; in contrast, the three profiles were consistent for women across all three timepoints. 
The results of this research suggest that there is considerable overlap in the theoretical orientation defining the treatment typologies that emerged for both justiceinvolved men and women. Consequently, similar to previous research (e.g., Brennan et al., 2012, Greiner, 2015; Jones et al., 2014), the findings highlight the importance of theoretical integration - that is, combining gender-neutral, gender-responsive, and strengths-based perspectives to understand the variability among justice-involved men and women. The development of treatment typologies can help inform effective service delivery, including programming and supervision practices, which can be tailored in accordance. Future research is encouraged to replicate these findings and examine treatment typologies among higher risk justice-involved samples. 


\section{Chapter 5}

\section{General Discussion}

The examination of offender risk factors has guided the criminal justice field, with research focusing on theories of offending that are used to explain the role of risk factors in understanding criminal behaviour among men and women. The main theoretical model used to inform processes within the criminal justice system (such as risk assessment, case management planning, and treatment targets) is the risk, need, and responsivity (RNR; Bonta \& Andrews, 2017) model. Until recently, the RNR model was almost exclusively deficit-based. However, in the latest version of the model, the role of strengths has been highlighted (Bonta \& Andrews, 2017). Over the past 20 years, strengths-based perspectives have emerged in the literature, including the good lives model (GLM; Ward, 2002; Ward \& Stewart, 2003), whereby the focus is on encouragement and developing offender skills rather than focusing on mitigating risk factors. Unfortunately, how these two perspectives coincide is less known. Ultimately, the majority of research has focused on the validation of the RNR model with very little research examining the importance of strengths (although research is emerging; e.g., Jones et al., 2015). In addition, research that has been conducted on both risk and strengths in correctional settings focusing predominately on men-which is not entirely surprising, given the higher prevalence of men in the correctional system. As such, a third perspective that must be considered is a gender-responsive perspective to offending behaviour, in particular, pathways theory (Belknap, 2015; Chesney-Lind, 1997). This perspective posits that traditional correctional theories fail to consider the unique needs of 
women, although a lot less research has been conducted examining gender differences (and similarities) using samples comprised of both men and women.

One of the major areas of importance for offenders in the criminal justice system is risk assessment. Risk assessment is used to inform every decision in an offender's progression through the justice system, including security classification, sentencing, treatment and case management planning, and predicting the likelihood (or risk) of a negative outcome (Hanson \& Morton-Bourgon, 2009). While the focus of risk assessments has predominately been on static risk factors (which are found to be predictive of reoffending, but unable to assess offender change), research on dynamic risk factors is increasing (Hanson \& Harris, 2001; Wong, Olver, \& Stockdale, 2009). Specifically, while research has demonstrated the importance of assessing dynamic risk factors (e.g., Simourd, 2004), little is known about how dynamic risk factors change over time (Brown et al., 2009; Lloyd, 2015). There is also limited research on how the assessment of strengths operate in risk assessment protocols and how dynamic strengths change over time (Wanamaker et al., 2018).

For the purpose of this dissertation, a gender-informed risk and strength assessment and case management tool, the Service Planning Instrument (SPIn; Orbis Partners, 2003), was used to further examine the role of dynamic strength and dynamic risk factors in predicting reoffending outcomes (including technical violations, any new charges, violent charges, and any negative outcome), to examine how offenders change over time, and to assess how various dynamic risk and strength domains coincide to inform treatment profiles of offenders. In addition, gender differences (and similarities) were considered at each set of analyses. 


\section{Overview of Key Results}

Given the limited research on dynamic risk and strength factors for both men and women, the first goal of this dissertation was to examine how SPIn-assessed dynamic factors change over time. As such, Study 1 was comprised of two main parts; first, the pattern of change in dynamic risk and strength factors and how these changes may differ between men and women were examined; this was followed by an examination of the relationship between change in scores over time, and reoffending outcomes.

Another area that is worth further exploration is typological research, which has predominately focused on one gender at a time and at one timepoint, and often excludes strength factors in the typological development. As such, the second goal of this dissertation was to examine theoretically integrated typologies of justice-involved men and women that merge tenets of gender-neutral, gender-responsive, and strengths-based paradigms. Study 2 examined whether typologies could be delineated among men and women, whether these typologies were stable over time, and whether there were gender differences in typological membership. Further, whether typological membership was stable over time and the relationship to reoffending outcomes was also examined.

Summary of Study 1 results. To account for the large sample of men in comparison to women, separate change models were assessed for men and women, and for both total dynamic risk and total dynamic strength, thus resulting in four change models. Overall results of both dynamic risk and strength models were similar for men and women. The initial total dynamic score and the initial dynamic strength score were both slightly higher for the men than the women, although the rate of change was similar (for both genders and for both risk and strength models). In each model, three covariates 
were included - age, Indigenous status, and static risk, to assess whether they explained variation in scores. The results were largely the same for both genders with a few notable gender differences.

First, for women, only the total static risk score explained significant variability in the initial and change over time in total dynamic risk scores. Specifically, women with higher static risk scores had higher dynamic risk scores at initial assessment, and had faster rates of change (decrease in scores) over time. For men, however, each of the covariates explained significant variability in initial and change scores (with the exception of Indigenous status which did not influence initial scores on total dynamic risk). In contrast, for strengths, while each covariate significantly influenced total dynamic strength scores for the men, Indigenous status did not influence initial strength score for the women. However, for both men and women, each covariate significantly influenced change in strength scores over time, with Indigenous status having the strongest influence. Therefore, while Indigenous men had higher initial strength scores, the rate of change (increase in scores) was slightly faster for non-indigenous men. For women, both Indigenous and non-Indigenous women had similar initial strength scores, however, the rate of change (increase in scores) was slightly faster for non-Indigenous women. Overall, the results indicated minimal and gradual change for both men and women and for both dynamic risk and strength scores.

The second goal of this study was to assess how change scores in both dynamic risk and strength scores predicted four criminal outcomes; technical violations, any new charges, violent charges, and any negative outcome. The prediction results for dynamic risk and strength models were quite similar for both genders. For the total dynamic risk 
models, initial scores were predictive of all four outcomes, for both men and women. In comparison, for the rate of change in total dynamic risk scores, the change score was predictive of all four outcomes for men. However, for women it was not predictive of any new violent charge (but was predictive of the three other outcomes). For the strengths models, the initial assessment scores were predictive of any new charge and technical violations for men, but not for violent charges or any negative outcome. For women, initial strength scores were predictive of all outcomes with the exception of any new charges. Although there were some differences in prediction of initial strengths scores for men and women, the prediction results of the change in strengths scores were similar for both genders, whereby change in total dynamic strength scores were only predictive of technical violations.

Summary of Study 2 results. To account for the large sample of men in comparison to women, latent profile analysis and latent transition analysis were conducted for men and women independently. For the men, a five-profile structure emerged for Time 1 and Time 2 made up of the following typologies: Low need/low strength; Aggressive, complex need/low strength; Moderate risk/moderate strength; Low risk/high strength; and Low stability, complex need/low strength. At the third wave, however, a sixth profile also emerged (i.e., Moderate complex need/low strength). This sixth profile that emerged could be a result of men in typologies with high needs in the previous timepoints (including the Aggressive, complex need/low strength profile, and the Low stability, complex need/low strength profile) decreasing in dynamic needs over time. Perhaps the dynamic risk factors of these men decreased over time, however, because the childhood adversity score is comprised of items that are static in nature, it remained to be 
a defining factor. In other words, it is entirely possible that men decreased from high need to moderate need over time, with the exception of childhood adversity; thus, creating a new typology at Time 3 .

For women, the same profile structure emerged across timepoints, which consisted of three profiles: Low risk/low strength; Gender-responsive, low risk/high strength; and Aggressive, complex need/low strength. These results demonstrate that there are typologies that are similar for both men and women. In fact, two typologies that emerged for women, also emerged for men (i.e., Low risk/low strength; and Aggressive, complex need/low strength), however, for men, there was an additional three typologies that emerged. The reason these additional typologies emerged for men may predominately be due to the large sample size (there were approximately 5,000 more men than women, which could explain the additional variability in typologies). It is also important to note that when comparing the domain scores in the typologies, the dynamic risk and strength domains are deemed 'high' and 'low' relative to the rest of the sample and are not deemed 'high' or 'low' based on cut off scores provided by the SPIn scoring guidelines. Regardless, these typologies that emerged have highlighted the importance of merging gender-neutral, gender-responsive, and strengths-based paradigms for both men and women.

Finally, Study 2 found that for women the profile with the highest rate of reoffending was the Aggressive, complex need/low strength profile, however, the other two profiles reoffended at comparable rates - indicating that women who were low risk but had gender-responsive needs did not differ from low risk women (without genderresponsive needs), if they scored high on strengths. Similarly, for the men, the three 
classes that reoffended the most were the Aggressive, complex need/low strength profile, the Moderate complex need/low strength, and the Low stability, complex need/low strength profile, indicating that childhood adversity and mental health issues may play an important role in understanding men's offending behaviour — similar to women.

\section{Implications for Theory and Research}

Three main paradigms informed this dissertation; a gender-responsive perspective (i.e., pathways theory), a gender-neutral perspective (i.e., the RNR model), and a strengths-based perspective. Pathways theory posits that traditional theories of crime are androcentric in nature, thus inadequate in explaining the reasons why women engage in criminal behaviour (Belknap, 2015; Belknap \& Holsinger, 2008; Chesney-Lind, 1997). In contrast, the RNR model posits that there are key factors that explain criminal behaviour for all offenders, irrespective of gender (Andrews et al., 2012; Bonta \& Andrews, 2017). Finally, the strengths-based perspective (such as the Good Lives Model; Ward \& Stewart, 2003) posits that strengths play a crucial role in explaining behaviour and that deficitbased theories of criminal behaviour (e.g., RNR) do not provide the full picture. Given that there has been a lack of research examining the potential gender differences (and similarities) using a gender-informed approach — that is, informed by multiple theoretical perspectives that include both genders - and incorporating strengths, the findings from the current studies suggest that integration of these three paradigms is essential for obtaining a holistic understanding of offending behaviour for both men and women.

The results of Study 1 suggest that men and women are in fact more similar than they are different, particularly in terms of identified risks and needs on risk assessments, which has been supported by previous research suggesting that men and women are in 
fact quite similar in most areas of their lives (e.g., Hyde, 2005). In terms of dynamic risk and dynamic strengths scores, both initial scores and change in scores over time were similar for men and women. This study has also highlighted the importance of considering strengths in risk assessment protocols, particularly for the purpose of predicting reoffending, which ties in with previous research indicating that strengths are predictive of future offending behaviour (e.g., Jones et al., 2015; Lloyd, 2015).

The results of Study 2 suggest that, despite slight gender differences, men and women are more similar than different in terms of their treatment profiles, whereby the risk factors and strengths that are useful for classifying offender typologies are a combination of gender-responsive and gender-neutral items; that is, gender-informed factors were useful for determining the profile structure of both men and women. Importantly, there were typologies that emerged for both genders that were comprised of both gender-neutral and gender-responsive risk factors; and other typologies that also comprised high strengths. As such, this lends support for the integration of genderneutral, gender-responsive, and strengths-based perspectives for the purpose of identifying unique profiles of justice-involved men and women.

Offender change research and research focusing on strengths are areas that require more attention, as both are in their infancy. This includes further exploration of how dynamic risk factors change over time across various subpopulations and risk classification levels, as well as more research focusing on the role that strengths play across various stages of the criminal justice system; including risk assessment, security classification, case management planning, and developing treatment targets. Additional areas that require further attention that was beyond the scope of the current dissertation, 
but are nonetheless important; offenders who identify as gender non-binary and the importance of intersectionality. Future research is encouraged to further extend the examination of gender beyond the dichotomous cis men and cis women definition. That is, future correctional research should include those who identify as gender non-binary, such as individuals who are gender-fluid, demi-gendered, transgendered, and bigendered. By failing to conduct research on individuals who do not identify as cis men or cis women, the criminal justice system may be failing to meet the needs of these individuals, leading to further disadvantage.

Another key area for research is the inclusion of intersectionality whereby the unique needs of disadvantaged individuals on the basis of gender are also examined in conjunction with other areas of oppression and discrimination including ethnicity, sexual orientation, and socio-economic status. Simply focusing on one dimension of discrimination or vulnerability does not provide the full context around the unique experiences, and thus unique needs of these individuals.

\section{Implications for Policy and Practice}

The results of the current study highlight the importance of re-assessing dynamic risk scores. Specifically, results demonstrated that change scores were more predictive than initial scores for both men and women and across all outcomes - with the exception of violent charges for women. Change in strength scores, in contrast while not as predictive as change in dynamic risk scores, were predictive of technical violations, and this relationship was stronger for women. Although further research is required to examine whether changes in strengths add incrementally to the predictability of change in dynamic risk scores, these findings still highlight the importance of incorporating 
strengths into risk assessment protocols. Assessing both risks and strengths provides a holistic approach to assessment protocols, which translates to more comprehensive case management planning and treatment.

The findings from Study 1 and Study 2 highlight the need for holistic treatment, that is both strengths-based and trauma-informed, for both men and women. While Study 1 highlighted the importance of strengths in predicting offending outcomes for men and women, Study 2 demonstrated that many gender-responsive factors, such as childhood adversity, are important for developing treatment typologies for men and women. Throughout the literature, correctional researchers have argued that because victimization experiences are highly prevalent among justice-involved women, trauma-informed services should be combined with correctional programming (Covington, 2016; Dehart, 2018; King, 2017). Although the importance of incorporating trauma-informed and strengths-based correctional treatment for justice-involved women has repeatedly emerged throughout the literature, there has been limited research on trauma-informed services with justice-involved men (with the exception of men sex offenders; see Janssen, 2018). Given that experiencing adverse childhood events is prevalent among both justiceinvolved boys and men (e.g., Wanamaker, Brown, \& Czerwinsky, 2020) and can lead to several issues, including problems maintaining relationships and behavioural problems (which can affect relationships with probation officers; Ford, Chapman, Connor, \& Cruise, 2012; Haider et al., 2018), more research is needed on the utility of incorporating trauma-informed services in programming for justice-involved men. 


\section{Limitations and Directions for Future Research}

The main limitation of the current research was the large amount of data lost and the limited generalizability due to the sample being predominately low risk men and women. As Lloyd (2015) noted, one of the main issues of assessing change over time on offender risk assessments is the amount of time between assessments (often several months apart) and the limited time to reoffending (often occurring within the first few months of start of supervision, reducing the number of assessments). Thus, to assess change, the current study removed anyone with less than three timepoints, and anyone who did not have reoffending information. In addition, anyone who did not have an initial SPIn assessment within 90 days of start of supervision was also removed - due to the fact that scores after this date may not be an adequate reflection of scores at the start of supervision (as change may have already occurred). Further, because high risk offenders are more likely to reoffend, and reoffend at a quicker rate post start of supervision, limiting the sample inclusion to only those with three timepoints reduces the sample to predominately low-risk cases. Because of this, results cannot be generalized to the entire sample of men and women on community supervision, but rather, is reflective of those who are more successful in the community, who either remain successful over time or who reoffend later on in their sentence (long-term reoffending). Nonetheless, this information provides useful information on whether change scores are predictable of reoffending outcomes, which provides support for not only assessing dynamic risk (and strengths) over time, but also the importance of targeting dynamic risk factors in treatment to promote change in dynamic factors. Future research is encouraged to examine change in risk and strength scores using high risk samples of men and women, 
as well as focus on specific dynamic risk and strength factors to determine how they change over time, including which factors are more stable versus acute in nature to further inform correctional treatment and case management planning.

\section{Summary}

This research was the first to examine how gender-informed, SPIn-assessed dynamic risks and strengths changed over time and link these changes to subsequent reoffending outcomes. This research also examined the gender differences and similarities in treatment profiles of men and women on community supervision using a gender-neutral, gender-responsive, and strengths-based factors. By combining these three theoretical perspectives, this study concludes that there are more similarities than differences among men and women in terms of changes in dynamic risks and strengths over time and typological structures. This was one of the first multi-wave longitudinal designs in correctional-based research to assess change in total dynamic risk and strength scores using the two-stage hierarchical linear modeling approach. Results highlight the importance of utilizing a holistic approach to offender risk assessment and treatment efforts. That is, programming is encouraged to incorporate a trauma-informed, strengthsbased approach to offender rehabilitation for both men and women. Future research is encouraged to further examine changes in dynamic risks and strengths among high-risk samples of justice-involved men and women. 


\section{References}

Allport, G. W. (1961). Pattern and growth in personality. New York: Holt, Rinehart \& Wilson.

Andersen, M. L., \& Collins, P. H. (2004). Race, class, and gender: An anthology (5th ed.). Belmont, CA: Wadsworth.

Andrews, D. A. (2012). The risk-need-responsivity (RNR) model of correctional assessment and treatment. In J. A. Dvoskin, J. L. Skeem, R. W. Novaco, \& K. S. Douglas (Eds.), American psychology-law society series. Using social science to reduce violent offending (pp. 127-156). New York, NY: Oxford University Press.

Andrews, D. A., \& Bonta, J. (2010). The psychology of criminal conduct ( $5^{\text {th }}$ ed.). New Providence, NJ: LexisNexis Matthew Bender.

Andrews, D. A., Bonta, J., \& Hoge. R. (1990). Classification for effective rehabilitation: Rediscovering psychology. Criminal Justice Behaviour, 17, 19-52. doi: $10.1177 / 0093854890017001004$

Andrews, D. A., Bonta, J., \& Wormith, J. S. (1995). The Level of Service Inventory: Ontario Revision. Toronto, ON: Ontario Ministry of Correctional Services

Andrews, D. A., Bonta, J., \& Wormith, J. S. (2004). The Level of Service / Case Management Inventory (LS/CMI): User's manual. Toronto, ON: Multi-Health Systems.

Andrews, D. A., Bonta, J., \& Wormith, S. J. (2011). The risk-need-responsivity (RNR) model: Does adding the good lives model contribute to effective crime prevention? Criminal Justice and Behavior, 38, 735-755. doi:

$10.1177 / 0093854811406356$ 
Andrews, D.A., Guzzo, L., Raynor, P., Rowe, R. C., Rettinger, J., Brews, A., \& Wormith, J. S. (2012). Are the major risk/need factors predictive of both female and male reoffending?: A test with the eight domains of the level of service/case management inventory. International Journal of Offender Therapy and Comparative Criminology, 56, 113-133. doi: 10.1177/0306624x10395716

Andrews, D. A., \& Robinson, D. (1984). The level of supervision inventory: Second report. Ottawa, ON: Community Division of the Ontario Ministry of Correctional Services.

Andrews, D. A., \& Wormith, J. S. (1984). Criminal sentiments and criminal behaviour. Programs Branch User Report. Ottawa, ON: Solicitor General Canada.

Anthony, E. J. (1974). The syndrome of the psychologically invulnerable child. In E. J. Anthony \& C. Koupernik (Eds.), The child in his family: Children at psychiatric risk (pp. 529-545). New York: Wiley.

Asparouhov, T., \& Muthén, B. (2009). Exploratory structural equation modeling. Structural Equation Modeling, 16, 397-438. doi: 10.1080/10705510903008204

Babchishin, K. M. (2013). Sex offenders do change on risk-relevant propensities: Evidence from a longitudinal study of the ACUTE-2007. (Unpublished dissertation). Ottawa, ON, Canada: Carleton University.

Baca Zinn, M., \& Thornton Dill, B. (1996). Theorizing difference from multiracial feminism. Feminist Studies, 22, 321-331.

Baglivio, M. T., Wolff, K. T., Piquero, A. R., \& Epps, N. (2015). The relationship between Adverse Childhood Experiences (ACE) and juvenile offending 
trajectories in a juvenile offender sample. Journal of Criminal Justice, 43, 229241. doi: 10.1016/j.jcrimjus.2015.04.012

Baird, C. S. (2009). A question of evidence: A critique of risk assessment models used in the justice system. Madison, WI: National Council on Crime and Delinquency.

Bakker, L., O’Malley, J. \& Riley, D. (1998). Storm warning: Statistical models for predicting violence. Christchurch, New Zealand: Psychological Service, Department of Corrections.

Barnao, M., Robertson, P., \& Ward, T. (2010). The Good Lives Model applied to a forensic population. Psychiatry, Psychology, \& Law, 17, 202-217. doi: $10.1080 / 13218710903421274$

Barnes, G. C., Ahlman, L., Gill, C., Sherman, L. W., Kurtz, E., \& Malvestuto, R. (2010). Low intensity community supervision for low risk offenders: A randomized, controlled trial. Journal of Experimental Criminology, 6, 159-189. doi: $10.1007 /$ s1 1292-010-9094-4

Barnoski, R. P. (2003). Evaluation of Washington State's 1996 Juvenile Court Program for High-risk, First-time Offenders: Final Report. Washington State Institute for Public Policy

Bartels, L. (2012). Violent offending by and against Indigenous women. Indigenous Law Bulletin, 8(1), 19-22.

Belknap, J. (2015). The invisible woman: Gender, crime and justice (4 ${ }^{\text {th }}$ ed.). Stamford, CT: Cengage Learning.

Belknap, J., \& Holsinger, K. (2006). The gendered nature of risk factors for delinquency. Feminist Criminology, 1, 48-71. doi: 10.1177/1557085105282897 
Bell, B. A., Ene, M., Smiley, w., \& Schoeneberger, J. A. (2013). A multilevel model primer using SAS PROC MIXED. SAS Global Forum. University of South Carolina.

Birgden, A. (2002). Therapeutic jurisprudence and "good lives": A rehabilitation framework for corrections. Australian Psychologist, 37(3), 180-186.

Blanchette, K. D., \& Brown, S. L. (2006). The assessment and treatment of women offenders: An integrative perspective. Wiley Series in Forensic Clinical Psychology. Chichester: John Wiley and Sons.

Bloom, B., Owen, B., \& Covington, S. (2003). Gender-responsive strategies - Research, practice, and guiding principles for women offenders. Washington: National Institute of Corrections.

Blozis, S. A., \& Cho, Y. I. (2008). Coding and centering of time in latent curve models in the presence of interindividual time heterogeneity. Structural Equation Modeling, 15(3), 413-433. doi: 10.1080/10705510802154299

Bonta, J. (1996). Risk-needs assessment and treatment. In A. T. Hartland (Ed.), Choosing correctional options that work: Defining the demand and evaluating the supply (pp. 18-32). Thousand Oaks: Sage Publications

Bonta, J. (2002). Offender risk assessment: Guidelines for selection and use. Criminal Justice and Behaviour, 29, 355-379.

Bonta, J. (2009). What to do with low risk offenders? Research Summary, 14(4).

Retrieved from https://www.publicsafety.gc.ca/cnt/rsrcs/pblctns/tdwthlwrsk/tdwth-lwrsk-eng.pdf 
Bonta, J., \& Andrews, D. A. (2003). A commentary on Ward and Stewart's model of human needs. Psychology, Crime, \& Law, 9, 215-218. doi: $10.1080 / 10683 / 16031000112115$

Bonta, J., \& Andrews, D. A. (2017). The psychology of criminal conduct (6 $6^{\text {th }}$ ed.). New York, NY: Routledge Taylor \& Francis.

Bonta, J., Blais, J., \& Wilson, H. A. (2013). The Prediction of Riskfor Mentally Disordered Offenders: A Quantitative Synthesis. Corrections Research. Ottawa, ON, Canada: Public Safety Canada.

Bonta, J., Bourgon, G., Rugge, T., Scott, T. L., Yessine, A. L., Gutierrez, L., \& Li, J. (2011). An experimental demonstration of training probation officers in evidencebased community supervision. Criminal Justice and Behavior, 38, 1127-1148. doi: $10.1177 / 0093854811420678$.

Bonta, J., Law, M., \& Hanson, R. K. (1998). The prediction of criminal and violent recidivism among mentally disordered offenders: A meta-analysis. Psychological Bulletin, 123, 123-142. doi: 10.1037/0033-2909.123.2.123

Bonta, J., Rugge, T., Bourgon, G., \& Wanamaker, K. A. (2019). A conceptual replication of the Strategic Training Initiative in Community Supervision (STICS). Journal of Experimental Criminology, 15, 397-419. doi: 10.1007\%2Fs11292-019-09371-4

Bourgon, G., Mugford, R., Hanson, R. K., \& Coligado, M. (2018). Offender risk assessment practices vary across Canada. Canadian Journal of Criminology and Criminal Justice, 60(2), 167-205. doi: 10.3138/cjccj.2016-0024

Brennan, T. (2008). Explanatory diversity among female delinquents: Examining taxonomic heterogeneity. In R. T. Zaplin (Ed.), Female offenders: Critical 
perspectives and effective interventions ( $2^{\text {nd }}$ ed., pp. 197-232). Boston: Jones and Bartlett.

Brennan, T., Breitenbach, M., \& Dieterich, W. (2008). Towards an explanatory taxonomy of adolescent delinquents: Identifying several social-psychological profiles. Journal of Quantitative Criminology, 24, 179-203.

Brennan, T., Breitenbach, M., Dieterich, W., Salisbury, E. J., \& Van Voorhis, P. (2012). Women's pathways to serious and habitual crime: A person-centered analysis incorporating gender responsive factors. Criminal Justice and Behavior, 39, 14811508.

Brown, G. P., Barker, J., McMillan, K., Norman, R., Derkzen, D., Stewart, L. A., \& Wardrop, K. (2018). Prevalence of mental disorder among federally sentenced women offenders: In-Custody and intake samples (Research Report R-420). Ottawa, ON: Correctional Service Canada.

Brown, S. L., St. Amand, M., \& Zamble, E. (2009). The dynamic prediction of criminal recidivism: A 3-wave prospective study. Law and Human Behavior, 33, 35-45. doi: 10.1007/s10979-008-9139-7

Brown, S. L., Wanamaker, K. A., Greiner, L., Scott, T., \& Skilling, T. A. (2020). Complex trauma and criminogenic needs: Two paths to the youth justice systemA gender-informed latent profile analysis (Submitted Manuscript).

Brown, S. L., Wanamaker, K. A., Wagstaff, M., \& Robinson, D. (2020). Strengths Matter: Evidence from Five Separate Cohorts of Justice-involved Youth and Adults Across North America (Submitted Manuscript). 
Brushett, R. A. (2013). Typologies of female offenders: A latent class analysis using the women's risk needs assessment. (Unpublished dissertation). University of Cincinnati: Cincinnati, Ohio, USA.

Burgess-Proctor, A. (2006). Intersections of race, class, gender, and crime: Future directions for feminist criminology. Feminist Criminology, 1, 27-47. doi: $10.1177 / 1557085105282899$

Cattell, R. B. (1966). The Scree Plot Test for the Number of Factors. Multivariate Behavioral Research, 1, 140-161.

Cerny, B. A., \& Kaiser, H. F. (1977). A study of a measure of sampling adequacy for factor analytic correlation matrices. Multivariate Behavioral Research, 12, 43-47. doi: $10.1207 / \mathrm{s} 15327906 \mathrm{mbr} 1201 \_3$

Charak, R., Ford, J. D., Modrowski, C. A., Kerig, P. K. (2019). Polyvictimization, emotion dysregulation, symptoms of Posttraumatic Stress Disorder, and behavioral health problems among justice-involved youth: A latent class analysis. Journal of Abnormal Child Psychology, 47(2), 287-298. doi: 10.1007/s10802018-0431-9

Chesney-Lind, M. (1989). Girls' crime and woman's place: Toward a feminist model of female delinquency. Crime and Delinquency, 35, 5-29. doi:

$10.1177 / 0011128789035001002$

Chesney-Lind, M. (1997). The female offender: Girls, women and crime. Thousand Oaks, CA: Sage.

Chesney-Lind, M., \& Shelden, R. G. (2003). Girls, delinquency, and juvenile justice (3 ${ }^{\text {rd }}$ ed.). Belmont, CA: Wadsworth Publishing Company. 
Chesney-Lind, M., \& Pasko, L. (2013). The female offender: Girls, women and crime $\left(3^{\text {rd }}\right.$ ed). California: Sage Publications.

Chung, I. J., Hill, K. G., Hawkins, J. D., Gilchrist, L. D., \& Nagin, D. S. (2002).

Childhood predictors of offense trajectories. Journal of Research in Crime and Delinquency, 39(1), 60-90. doi: 10.1177/002242780203900103

Clarke, M., Peterson-Badali, M., \& Skilling, T. (2019). Patterns of change in dynamic risk factors over time in youth offenders. Canadian Journal of Criminology and Criminal Justice, 61(2), 1-25. doi: 10.3138/cjccj.2018-0001

Cohen, T. H., Cook, D. L., \& Lowenkamp, C. T. (2016). The supervision of low-risk offenders: How the low-risk policy has changed federal supervision practices without compromising community safety. Federal Probation, 80(1), 3-11.

Cohen, T. H., Lowenkamp, C. T., \& VanBenschoten, S. W. (2016). Does change in risk matter? Examining whether changes in offender risk characteristics influence recidivism outcomes. Criminology \& Public Policy, 15(2), 263-296. doi: $10.1111 / 1745-9133.12190$

Collins, L. M., \& Lanza, S. T., (2010). Latent class and latent transition analysis for the social, behavioural, and health sciences. New York: Wiley.

Copas, J. B. (1997). Using regression models for prediction: Shrinkage and regression to the mean. Statistical Methods in Medical Research, 6(2), 167-183. doi: $10.1177 / 096228029700600206$

Cottle, C. C., Lee, R. J., \& Heilbrun, K. (2001). The prediction of criminal recidivism in juveniles: A meta-analysis. Criminal Justice and Behavior, 28, 367-394. doi: $10.1177 / 0093854801028003005$ 
Covington, S. (2013). Beyond Violence: A Prevention Program for Criminal JusticeInvolved Women. Hoboken, NJ: Wiley.

Covington, S. (2016). Beyond Trauma: A Healing Journey for Women (2nd ed.). Center City, MN: Hazelden Publishing.

Covington, S. (2019). Helping Women Recover: A Program for Treating Addiction. Special Edition for Use in the Criminal Justice System (3rd ed.). San Francisco, CA: Jossey-Bass.

Covington, S., \& Bloom, B. E. (2006). Gender-responsive treatment and services in correctional settings. In E. Leeder (Ed.), Inside and out: Women, prison, and therapy (pp. 9-34). Binghamton, NY: Haworth Press.

Daly, K. (1992). Women's pathways to felony court: Feminist theories of lawbreaking and problems of representation. Review of Law and Women's Studies, 2(11), 1152.

Daly, K. (1994). Gender, crime, and punishment. New Haven, CT: Yale University Press.

Daly, K., \& Tonry, M. (1997). Gender, race, and sentencing. Crime and Justice, 201-252

Dehart, D. D. (2008). Pathways to prison: impact of victimization in the lives of incarcerated women. Violence Against Women, 14, 1362-1381. doi: $10.1177 / 1077801208327018$

Dehart, D. D. (2018). Women's pathways to crime: A heuristic typology of offenders. Criminal Justice and Behavior, 45, 1461-1482. doi: 10.1177/0093854818782568

Dembo, R., \& Schmeidler, J. (2003). A classification of high-risk youth. Crime \& Delinquency, 42, 201-230. doi: 10.1177/0011128702251054 
Dembo, R., Wareham, J., Poythress, N., Meyers, K., Cook, B., \& Schmeidler, J. (2008). Psychosocial functioning problems over time among high-risk youth: A latent class transition analysis. Crime and Delinquency, 54, 664-670. doi: $10.1177 / 0011128707306016$

Dennis, J. P. (2014). The LGBT Offender. In D. Peterson \& V. Panfil (Eds.), Handbook of LGBT Communities, Crime and Justice (pp. 87 - 102). Springer.

Dickens, G. L., \& O'Shea, L. E. (2018). Protective factors in risk assessment schemes for adolescents in mental health and criminal justice populations: A systematic review and meta-analysis of their predictive efficacy. Adolescent Research Review, 3, 95-112. doi: 10.1007/s40894-017-0062-3

Dolan, M. C., \& Rennie, C. E. (2008). The Structured Assessment of Violence Risk in Youth as a predictor of recidivism in a United Kingdom cohort of adolescent offenders with conduct disorder. Psychological Assessment, 20(1), 35-46. doi: $10.1037 / 1040-3590.20 .1 .35$

Douglas, K. S., \& Skeem, J. L. (2005). Violence risk assessment: Getting specific about being dynamic. Psychology, Public Policy, and Law, 11, 347-383. doi: $10.1037 / 1076-8971.11 .3 .347$

Dowden, C., \& Andrews, D. A. (1999). What works for female offenders: A metaanalytic review. Crime and Delinquency, 45(4) 438-452.

Dowden, C., \& Andrews, D. A. (2000). Effective correctional treatment and violent reoffending: A meta-analysis. Canadian Journal of Criminology, 42, 449-476. 
Dowden, C., \& Brown, S. L. (2002). The role of substance abuse factors in predicting recidivism: A meta-analysis. International Journal of Crime, Psychology, and Law, 8, 243-264. doi: 10.1080/10683160208401818

Dowden, C., Serin, R. C., \& Blanchette, K. D. (2001). The application of the Community Intervention Scale to women offenders: Preliminary findings (Research Report R97). Ottawa, ON: Research Branch, Correctional Service of Canada.

Ellerby, L., \& MacPherson, P. (2002). Exploring the profiles of Aboriginal sexual offenders: Contrasting Aboriginal and non-Aboriginal sexual offenders to determine unique client characteristics and potential implications for sex offender assessment and treatment strategies (Research Report R-122). Ottawa, ON: Correctional Service of Canada.

Farrington, D. P. (2003). Key results from the first forty years of the Cambridge Study in Delinquent Development (pp. 137-183). In T. P Thornberry and M. D. Krohn (Eds.) Taking Stock of Delinquency. Kluwer Academic/Plenum: Ney York, NY.

Farrington, D., Ttofi, M. M., \& Piquero, A. R. (2016). Risk, promotive, and protective factors in youth offending: Results from the Cambridge study in delinquent development. Journal of Criminal Justice, 45, 63-70. doi:

10.1016/j.jcrimjus.2016.02.014

Fedock, G., \& Covington, S. S. (2018). Strengths-based approaches to the treatment of female offenders. Retrieved online from: https://www.stephaniecovington.com/assets/files/8-3018\%20Revised $\% 20$ Fedock $\% 20$ Covington $\% 20$ with $\% 20$ suggested $\% 20$ edits $\% 208$ \%2030\%202018.pdf 
Felitti, V. J., Anda, R. F., Nordenberg, D., Williamson, D. F., Spitz, A. M., Edwards, V., ... Marks, J. S. (1998). Relationship of childhood abuse and household dysfunction to many of the leading causes of death in adults: The Adverse Childhood Experiences (ACE) Study. American Journal of Preventive Medicine, 14(4), 245-258. doi: 10.1016/S0749-3797(98)00017-8

Feng, C., Wang, H., Lu, N., Chen, T., He, H., Lu, Y., \& Tu, X. M. (2014). Logtransformation and its implications for data analysis. Shanghai archives of psychiatry, 26(2), 105-109. doi: 10.3969/j.issn.1002-0829.2014.02.009

Finkelhor, D., \& Baron, L. (1986). High-risk children. In D. Finkelhor (Ed.), $A$ sourcebook on child sexual abuse. Beverly Hills, CA: Sage Publications.

Finseth, S. M. L. (2020). Facilitating pathways out of crime: The role of strengths in risk assessment and rehabilitation among justice-involved youth. (Unpublished Dissertation). Toronto, ON: University of Toronto.

Fontaine, N., Carbonneau, R., Vitaro, F., Barker, E. D., \& Tremblay, R. E. (2009). Research review: A critical review of studies on the developmental trajectories of antisocial behavior in females. Journal of Child Psychology and Psychiatry, 50, 363-385. doi: 10.1111/j.1469-7610.2008.01949.x.

Ford, J. D., Chapman, J., Connor, D. F., \& Cruise, K. R. (2012). Complex trauma and aggression in secure juvenile justice settings. Criminal Justice and Behavior, 39(6), 694-724. doi: 10.1177/0093854812436957

Fowler, R. D., Seligman, M. E. P., \& Koocher, G. P. (1999). The APA annual report. American Psychologist, 54, 537-568. doi: 10.1037/0003-006x.54.8.537

Fox, T. M. K. (2019). Adversity, resilience, and crime: Examining the impact of 
resilience on the relationship between adverse childhood experiences and juvenile delinquency. (Unpublished Dissertation). Washington State University: Pullman, WA, USA.

Gable, S. L., \& Haidt, J. (2005). What (and why) is positive psychology? Review of General Psychology, 9, 103-110. doi: 10.1037/1089-2680.9.2.103

Garmezy, N. (1973). Competence and adaptation in adult schizophrenic patients and children at risk. In S. R. Dean (Ed.), Schizophrenia: The first ten Dean Award Lectures (pp. 168-204). New York: M. S. S. Information Corporation.

Garson, G. D. (Ed.). (2013). Hierarchical Linear Modeling: Guides and application. Thousand Oaks, CA: Sage Publications.

Gendreau, P., Little T., \& Goggin, C. (1996). A meta-analysis of the predictors of adult offender recidivism: What works! Criminology, 34, 575-607. doi: 10.1111/j.17459125.1996.tb01220.x

Gilfus, M. E. (1992). From victims to survivors to offenders: Women's routes of entry and immersion into street crime. Women \& Criminal Justice, 4, 63-90.

Glowacz, F., \& Born, M. (2015). Away from Delinquency and Crime: Resilience and Protective Factors. In J. Morizot \& L. Kazemian (Eds.)., The Development of Criminal and Antisocial Behavior: Theory, Research and Practical Applications. Switzerland: Springer International Publishing.

Gobeil, R., Blanchette, K., \& Stewart, L. (2016). A meta-analytic review of correctional interventions for women offenders - Gender-gender-neutral versus genderresponsive approaches. Criminal Justice and Behavior, 43, 301-322. doi: $10.1177 / 0093854815621100$ 
Golden-Biddle, K., \& Dutton, J. E. (Eds.). (2012). Using a positive lens to explore social change and organizations: Building a theoretical and research foundation. New York, NY: Routledge.

Green, L., \& Campbell, M. A. (2006). Gender influences and methodological considerations in adolescent risk-need assessment: A meta-analysis. Poster presented at the $67^{\text {th }}$ Annual Convention of the Canadian Psychological Association. Calgary, Alberta, Canada.

Greiner, L. (2013). A longitudinal examination of feminist pathways and mainstream correctional-informed risk factors using latent transition analysis and latent difference score modeling (Unpublished prospectus manuscript).

Greiner, L. E. (2015). Finding a middle ground: Advancing the feminist pathways paradigm through integration with "what works" with serious adolescent offenders. (Unpublished dissertation). Carleton University: Ottawa, ON.

Greiner, L., Law, M. A., \& Brown, S. L. (2015). Using dynamic factors to predict recidivism among women: A four-wave prospective study. Criminal Justice and Behavior, 42, 457-480. doi: 10.1177/0093854814553222

Gutierrez, L., \& Wanamaker, K. A. (2020). Risk assessment and Indigenous girls and women in the criminal justice system. In S. L. Brown \& L Gelsthorpe (Eds.), Evidence-Based Practices with Indigenous Girls and Women. West Sussex, UK: John Wiley \& Sons Limited.

Haider, A. H., Herrera-Escobar, J. P., Al Rafai, S. S., Harlow, A. F., Apoj, M., Nehra, D., ... Salim, A. (2018). Factors associated with long-term outcomes after injury. Annals of Surgery, 1, doi: 10.1097/SLA.0000000000003101 
Hanby, L. J. (2013). The relationship between dynamic risk, protective factors, and criminal recidivism in a New Zealand offender release cohort (Unpublished doctoral dissertation). Ottawa, ON: Carleton University.

Hannah-Moffat, K. (2009). Gridlock or mutability: Reconsidering "gender" and risk assessment. Criminology \& Public Policy, 8(1), 209-219. doi: 10.1111/j.17459133.2009.00549.x

Hanson, R. K., \& Harris, A. J. R. (1998). Dynamic predictors of sexual offence recidivism. (User Report 1998-01). Ottawa: Department of the Solicitor General Canada.

Hanson, R. K., \& Harris, A. J. R. (2001). A structured approach to evaluating change among sexual offenders. Sexual Abuse: A Journal of Research and Treatment, 13, 105-122. doi: 10.1177/107906320101300204

Hanson, R. K., Harris, A. J. R., Scott, T., \& Helmus, L. (2007). Assessing the risk of sexual offenders on community supervision: The Dynamic Supervision Project. User Report No. 2007-05. Ottawa, ON: Public Safety Canada.

Hanson, R. K., \& Morton-Bourgon, K. E. (2009). The accuracy of recidivism risk assessments for sexual offenders: A meta-analysis of 118 prediction studies. Psychological Assessent, 21, 1-21. doi: 10.1037/a0014421

Harris, G. T., \& Rice, M. E. (2015). Progress in violence risk assessment and communication: Hypothesis versus evidence. Behavioral Sciences and the Law, 33, 128-145. doi: 10.1002/bs1.2157

Healy, W., \& Bronner, A. F. (1936). New light on delinquency and its treatment: Results of a research conducted for the Institute of Human Relations Yale University. 
New Haven, CT: Yale University.

Helmus, L., Babchishin, K. M., \& Blais, J. (2012). Predictive accuracy of dynamic risk factors for Indigenous and non-Indigenous sex offenders: An exploration comparison using STABLE-2007. International Journal of Offender Therapy and Comparative Criminology, 56, 856-876. doi: 10.1177/0306624X11414693

Hoge, R. D., \& Andrews, D. A. (2003). The youth level of service/case management inventory (YCS/CMI): Intake manual and item scoring key. Ottawa, Ontario, Canada: Carleton University.

Howard, P. D., \& Dixon, L. (2013). Identifying change in the likelihood of violent recidivism: Causal dynamic risk factors in the OASys violence predictor. Law and Human Behavior, 37, 163-174. doi: 10.1037/1hb0000012

Hox, J. J. (2010). Multilevel analysis: Techniques and applications (2nd Ed.) NY: Routledge.

Hubbard, D. J., \& Pratt, T. C. (2002). A meta-analysis of the predictors of delinquency among girls. Journal of Offender Rehabilitation, 34, 1-13. doi:

10.1300/J076v34n03_01

Hyde, J. S. (2005). The gender similarities hypothesis. The American Psychologist, 60, 581-592. doi: 10.1037/0003-066X.60.6.581

IBM Corp. (Released 2017). IBM SPSS Statistics for Windows, Version 25.0. Armonk, NY: IBM Corp.

James, W. (1902). The varieties of religious experience: A study in human nature. New York: Longman, Green. 
Janssen, E. (2018). Integrating trauma informed care into the treatment of adult male sex offenders: A systematic review. Retrieved from Sophia, the St. Catherine University repository website: https://sophia.stkate.edu/msw_papers/826

Jones, N. J., Brown, S. L., Robinson, D., \& Frey, D. (2015). Incorporating strengths into quantitative assessments of criminal risk for adult offenders. Criminal Justice and Behavior, 42, 321-338. doi: 10.1177/0093854814547041

Jones, N. J., Brown, S. L., Robinson, D., \& Frey, D. (2016). Validity of the Youth Assessment and Screening Instrument: A Juvenile Justice Tool Incorporating Risks, Needs, and Strengths. Law and Human Behavior, 40, 182-194. doi: $10.1037 / \mathrm{lhb} 0000170$

Jones, N. J., Brown, S. L., Wanamaker, K. A., \& Greiner, L. (2014). A quantitative exploration of gendered pathways to crime in a sample of male and female juvenile offenders. Feminist Criminology, 9, 113-136. doi:

$10.1177 / 0093854809334076$

Jones, N. J., Brown, S. L., \& Zamble, E. (2010). Predicting criminal recidivism in adult male offenders: Researcher versus parole officer assessment of dynamic risk. Criminal Justice and Behavior, 37, 860-882. doi: 10.1177/0093854810368924

Jones, P. R. \& Harris, P. W. (1999). Developing an empirically based typology of delinquent youths. Journal of Quantitative Criminology, 15, 251-276. doi: $10.1023 / \mathrm{A}: 1007524414432$

Jones, N. J., \& Robinson, D. (2017a). The validation of the Service Planning Instrument (SPIn) for Alberta community corrections. Ottawa, ON: Orbis Partners Inc. 
Jones, N. J., \& Robinson, D. (2017b). The validity of the Service Planning Instrument for Clark County, Washington adult probation. Ottawa, ON: Orbis Partners Inc.

Jones, N. J., \& Robinson, D. (2018). Service Planning Instrument (SPIn). In J. P. Singh, D. G. Kroner, J. S. Wormith, S. L., Desmarais, \& Z. Hamilton (Eds.), Handbook of Recidivism Risk/Needs Assessment Tools ( $1^{\text {st }}$ ed.). John Wiley \& Sons Ltd.

King, E. A. (2017). Outcomes of trauma-informed interventions for incarcerated women: A review. International Journal of Offender Therapy and Comparative Criminology, 61, 667-688. doi: 10.1177/0306624X15603082

Kreuter, F., \& Muthén, B. (2008). Analyzing criminal trajectory profiles: Bridging multilevel and group-based approaches using growth mixture modeling. Journal of Quantitative Criminology, 24(1), 1-31.

Kruttschnitt, C. (2016). The politics, and place, of gender in research on crime: 2015 Presidential Address to the American Society of Criminology. Criminology, 54(1), 8-29. doi: 10.1111/1745-9125.12096

Labrecque, R. M., Smith, P. M., Lovins, B. K., \& Latessa, E. J. (2014). The importance of reassessment: How changes in the LSI-R risk score can improve the prediction of recidivism. Journal of Offender Rehabilitation, 53(2), 116-128. doi:

$10.1080 / 10509674.2013 .868389$

Laub, J. H., Nagin, D. S., \& Sampson, R. J. (1998). Trajectories of change in criminal offending: Good marriages and the desistance process. American Sociological Review, 63, 225-238. doi: 10.2307/2657324

Laws, D. R. (2002). Owning your own data: The management of denial. In M. McMurran (Ed.), Motivating offenders to change: A guide to enhancing engagement in 
therapy (pp. 173-191). Chichester, UK: Wiley.

Laws, D. R., \& Ward, T. (2011). Desistance and sexual offending: Alternatives to throwing away the keys. New York, NY: Guildford.

Lewis, K., Olver, M. E., \& Wong, S. C. (2013). The Violence Risk Scale: Predictive validity and linking changes in risk with violent recidivism in a sample of highrisk ofenders with psychopathic traits. Assessment, 20, 150-164. doi:

$10.1177 / 1073191112441242$

Lloyd, C. D. (2015). Can a dynamic risk instrument make short-term predictions in 'realtime'? Developing a framework for testing proximal assessment of offender recidivism risk during re-entry. (Unpublished dissertation). Ottawa, ON: Carleton University.

Lodewijks, H. P. B., de Ruiter, C., \& Doreleijers, T. A. H. (2010). The impact of protective factors in desistance from violent offending - A study in three samples of adolescent offenders. Journal of Interpersonal Violence, 25, 568-587. doi: $10.1177 / 0886260509334403$

Looman, J., \& Abracen, J. (2013). The risk need responsivity model of offender rehabilitation: Is there really a need for a paradigm shift? International Journal of Behavior and Consultation Therapy, 8, 30 -36. doi: 10.1037/h0100980

Lösel, F., \& Farrington, D. P. (2012). Direct protective and buffering protective factors in the development of youth violence. American Journal of Preventive Medicine, 43(2 supplement 1), S8-S23. doi: 10.1016/j.amepre.2012.04.029 
Lowenkamp, C. T., Holsinger, A. M., \& Latessa, E. J. (2001). Risk/need assessment, offender classification and the role of child abuse. Criminal Justice and Behavior, 28, 543-563. doi: 10.1177/009385480102800501

Lowenkamp, C. T., Latessa, E. J., \& Holsinger, A. M. (2006). The risk principle in action: What have we learned from 13,676 offenders and 97 correctional programs? Crime \& Delinquency, 52(1), 77-93. doi: 10.1177/0011128705281747

Lubke, G., \& Muthén, B. (2007). Performance of factor mixture models as a function of model size, covariate effects, and class-specific parameters. Structural Equation Modelling, 14, 26-47.

Magidson, J., \& Vermunt, J. K. (2004). Latent class models. In D. Kaplan (Ed.), The Sage handbook of quantitative methodology for the social sciences (pp. 175-198). Thousand Oaks: Sage Publication.

Malakieh, J. (2018). Adult and youth correctional statistics in Canada, 2016/2017. Juristat. Statistics Canada Catalogue No. 85-002-X.

Mann, R. E., Hanson, R. K., \& Thornton, D. (2010). Assessing risk for sexual recidivism: Some proposals on the nature of psychologically meaningful risk factors. Sexual Abuse: A Journal of Research and Treatment, 22, 191-217. doi: $10.1177 / 1079063210366039$

Marquart, J. W., Brewer, V. E., Simon, P., \& Morse, E. V. (2001). Lifestyle factors among female prisoners with histories of psychiatric treatment. Journal of Criminal Justice, 29, 319-328. 
Masten, A. S. (2013). Risk and resilience in development. In P. D. Zelazo (Ed.)., Oxford Handbook of Developmental Psychology (pp. 579-607). New York, NY: Oxford University Press.

McArdle, J. J. (2001). A latent difference score approach to longitudinal dynamic structural analyses. In R. Cudeck, S. du Toit, \& D. Sorbom (Eds.). Structural Equation Modeling: Present and future. Lincolnwood, IL: SSI. 342-380).

McCutcheon, A. L. (1987). Latent class analysis. Quantitative Applications in the Social Sciences Series No. 64. Thousand Oaks, California: Sage Publications.

Messina, N., Calhoun, S., \& Warda, U. (2012). Gender-responsive drug court treatment: A randomized controlled trial. Criminal Justice and Behavior, 39(12), 1539-1558. doi: $10.1177 / 0093854812453913$

Messina, N., Grella, C., Cartier, J., \& Torres, S. (2010). A randomized experimental study of gender responsive substance abuse treatment for women in prison. Journal of Substance Abuse Treatment, 38, 97-107.

Miller, H. A. (2006). Manual of the inventory of offender risks, needs, and strengths (IORNS). Odessa, FL: Psychological Assessment Resources.

Moffitt, T. E. (1993). 'Life-course persistent' and 'adolescent limited' antisocial behaviour: A developmental taxonomy. Psychological Review, 100, 674-701. doi: 10.1037/0033-295X.100.4.674

Morash, M. (2010). Women on probation and parole: A feminist critique of correctional programs and services. Boston, MA: Northeastern University Press.

Morgan, R. D., Kroner, D. G., Mills, J. F., Serna, C., \& McDonald, B. (2013). Dynamic risk assessment: A validation study. Journal of Criminal Justice, 41, 115-124. 
doi: 10.1016/j.jcrimjus.2012.11.004

Motiuk, L. L. (1999). Assessment methods in corrections. In P. M. Harris (Ed.), Research to results: Effective community corrections: Proceedings of the 1995 and 1996 conferences of the International Community Corrections Association (ICCA) (pp. 171-190). Lanham, MD: American Correctional Association

Muthén, L. K., \& Muthén, B. O. (1998-2017). Mplus User’s Guide. Eighth Edition. Los Angeles, CA: Muthén \& Muthén.

Nuffield, J. (1982). Parole decision-Making in Canada: Research Towards Decision Guidelines. Ottawa, ON: Solicitor General of Canada.

O'Brien, P. (2001). "Just like baking a cake": Women describe the necessary ingredients for successful reentry after incarceration. Families in Society: The Journal of Contemporary Social Services, 82(3), 287-295.

O’Connor, B. (2000). SPSS and SAS programs for determining the number of components using parallel analysis and Velicer's MAP test. Behavior Research Methods, Instruments, \& Computers, 32, 396-402. doi: 10.3758/BF03200807

Odgers, C. L., Moretti, M. M., Burnette, M. L., Chauhan, P., Waite, D., \& Reppucci, N. D. (2007). A latent variable modeling approach to identifying subtypes of serious and violent female juvenile offenders. Aggressive Behavior, 33, 339-352.

Office of the Correctional Investigator. (2015). Annual Report of the Office of the Correctional Investigator 2014-2015. Ottawa, ON: Minister of Public Works and Government Services Canada.

Ogloff, J. R. P., \& Davis, N. R. (2004). Advances in offender assessment and rehabilitation: Contributions of the risk-needs-responsivity approach. Psychology, 
Crime \& Law, 10, 229-242. doi: 10.1080/0683160410001662735

Olver, M. E., Stockdale, K. C., \& Wormith, J. S. (2009). Risk assessment with young offenders: A meta-analysis of three assessment measures. Criminal Justice and Behavior, 36(4), 329-353. doi: 10.1177/0093854809331457

Olver, M. E., Stockdale, K. C., \& Wormith, J. S. (2014). Thirty years of research on the Level of Service Scales: A meta-analytic examination of predictive accuracy and sources of variability. Psychological Assessment, 26, 156-176. doi: $10.1037 / \mathrm{a} 0035080$

Olver, M. E., Wong, S. C. P., Nicholaichuk, T., \& Gordon, A. (2007). The validity and reliability of the Violence Risk ScaleSexual Offender Version: Assessing sex offender risk and evaluating therapeutic change. Psychological Assessment, 19, 318-329. doi: 10.1037/1040-3590.19.3.318

Onifade, E., Nyandoro, A. S., Davidson, W. S., \& Campbell, C. (2010). Truancy and patterns of criminogenic risk in a young offender population. Youth Violence and Juvenile Justice, 8, 3-18. doi: 10.1177/1541204009338251

Orbis Partners. (2000). General Youth Assessment Screening Inventory (YASI). Ottawa, ON: Author.

Orbis Partners. (2003). Service Planning Instrument (SPIn). Ottawa, Ontario, Canada: Author.

Orrick, E. A. (2012). Criminal attitudes and re-entry: An analysis of post-release from prison among serious violent offenders (Master's thesis). Available from ProQuest Dissertations and Theses database. (UMI No. 3523601) 
Pasko, L. (2008). The wayward girl revisited: Understanding the gendered nature of juvenile justice and delinquency. Sociology Compass, 2(3), 821-836. doi: $10.1111 / \mathrm{j} .1751-9020.2008 .00093 . \mathrm{x}$

Perkins, K. R. (2010). Examining gender differences in typological membership using latent class analysis: A novel contribution to the gender-specificity/neutrality debate. (Unpublished dissertation). Carleton University: Ottawa, Ontario, Canada.

Perra, O. (2012). Latent transition analysis [PowerPoint slides]. Retrieved from http://www.restore.ac.uk/latentvariablemodels/workshopfiles/Latent\%20Transitio n\%20Analysis_Oliver\%20Perra/Course\%20Presentation\%20and\%20Exercises/L TA_course_UU.pdf

Piquero A. R. (2008). Taking stock of developmental trajectories of criminal activity over the life course. In Liberman A (ed), The Long View of Crime. Springer, New York (pp. 23-78).

Piquero, A. R., Reingle Gonzalez, J. M., \& Jennings, W. G. (2015). Developmental trajectories and antisocial behavior over the life-course. In Morizot J., and Kazemian L. (Eds.) The Development of Criminal and Antisocial Behavior. Springer, Cham.

Polaschek, D. L. L. (2012). An appraisal of the risk-need-responsivity (RNR) model of offender rehabilitation and its application in correctional treatment. Legal and Criminological Psychology, 17, 1-17. doi: 10.111/j.2044A8333.2011.02038.x

Polaschek, D. L. L. (2016). Desistance and dynamic risk factors belong together. Psychology, Crime, \& Law, 22(1-2), 171-189. 
Pusch, N., \& Holtfreter, K. (2018). Gender and risk assessment in juvenile offenders: A meta-analysis. Criminal Justice and Behavior, 45(1), 56-81. doi: $10.1177 / 0093854817721720$

Putnick, D. L., \& Bornstein, M. H. (2016). Measurement invariance conventions and reporting: The state of the art and future directions for psychological research. Developmental review, 41, 71-90. doi: 10.1016/j.dr.2016.06.004

Quinsey, V. L., Book, A. S., \& Skilling, T. A. (2004). A follow-up of deinstitutionalized men with intellectual disabilities and histories of antisocial behavior. Journal of Applied Research in Intellectual Disabilities, 17, 243-253. doi: $10.1111 / \mathrm{j} .14683148 .2004 .00216 . \mathrm{x}$

Quinsey, V. L., Coleman, G., Jones, B., \& Altrows, I. F. (1997). Proximal antecedents of eloping and reoffending among supervised mentally disordered offenders. Journal of Interpersonal Violence, 12, 794-813. doi: 10.1177/088626097012006002

Quinsey, V. L., Jones, G. B., Book, A. S., \& Barr, K. N. (2006). The dynamic prediction of antisocial behavior among forensic psychiatric patients: A prospective field study. Journal of Interpersonal Violence, 21, 1539-1565. doi:

$10.1177 / 0886260506294238$

Rao, M. A., \& Donaldson, S. I., (2015). Expanding opportunities for diversity in positive psychology: An examination of gender, race, and ethnicity. Canadian Psychology, 56, 271-282. doi: 10.1037/cap0000036

Raudenbush, S. W. \& Bryk, A. S. (2002). Hierarchical linear models: Applications and data analysis method (2 $2^{\text {nd }}$ Ed.). Newbury Park, CA: Sage. 
Rennie, C. E., \& Dolan, M. C. (2010). The significance of protective factors in the assessment of risk. Criminal Behaviour and Mental Health, 20, 8-22. doi: $10.1002 / \mathrm{cbm} .750$

Rettinger, L. J., \& Andrews, D. A. (2010). General risk and need, gender specificity, and the recidivism of female offenders. Criminal Justice and Behavior, 37, 29-46. doi: $10.1177 / 0093854809349438$

Rice, M. E., \& Harris, G. T. (2005). Comparing effect sizes in follow-up studies: ROC Area, Cohen's d, and r. Law and Human Behavior, 29, 615-620. doi: $10.1007 /$ s10979005-6832-7

Richie, B. E. (1996). Compelled to crime: The gender entrapment of Black battered women. New York: Routledge.

Rutter, M. (1979). Protective factors in children's responses to stress and disadvantage. In M. W. Kent \& J. E. Rolf (Eds.), Primary prevention of psychopathology: Vol. 3. Social competence in children (pp. 49-74). Hanover, NH: University Press of New England.

Salisbury, E., Boppre, B., \& Kelly, B. (2016). Gender-responsive risk and need assessment: Implications for the treatment of justice-involved women. In F. Taxman (Ed.). The Handbook on Risk and Need Assessment: Theory and Practice (pp. 220-243). Taylor \& Francis.

Salisbury, E. J., \& Van Voorhis, P. (2009). Gendered pathways: A quantitative investigation of women probationers' paths to incarceration. Criminal Justice and Behavior, 36, 541-566. doi: 10.1177/0093854809334076 
Salvatore, C., Taniguchi, T., \& Welsh, W. N. (2012). Is emerging adulthood influencing Moffitt's developmental taxonomy? Adding the "Prolonged" adolescent offender. Western Criminology Review, 13, 1-15.

Sampson, R. J., \& Laub, J. H. (2005). A life-course view of the development of crime. Annals of the American Academy of Political and Social Science, 602, 12-45. doi: $10.1177 / 0002716205280075$

SAS Institute Inc. (2016). Base $S A S \circledR 9.3$ Procedures Guide. Cary, NC: SAS Institute. Saskatchewan Ministry of Corrections. (2009). Saskatchewan Primary Risk Assessment: Scoring Manual. Saskatchewan: Public Safety and Policing, Adult Corrections

Scanlan, J. M., Yesberg, J. A., Fortune, C-A., \& Polaschek, D. L. L. (2020). Predicting women's recidivism using the Dynamic Risk Assessment for Offender Re-Entry: preliminary evidence of predictive validity with community-sentenced women using a "gender-neutral" risk measure. Criminal Justice and Behvaior, 47(3), 251270. doi: $10.1177 / 0093854819896387$

Schlager, M. D., \& Pacheco, D. (2011). Examination of changes in LSI-R scores over time: Making the case for needs-based case management. Criminal Justice and Behavior, 38, 541-553. doi: 10.1177/0093854811402300

Schwalbe, C. S. (2008). A meta-analysis of juvenile justice risk assessment instruments: Predictive validity by gender. Criminal Justice and Behavior, 35(11), 1367-1381. doi: $10.1177 / 0093854808324377$

Scott, T., \& Brown, S. L. (2018). Risks, strengths, gender, and recidivism among justiceinvolved youth: A meta-analysis. Journal of Consulting and Clinical Psychology, 86, 931-945. doi: 10.1037/ccp0000343 
Seligman, M. E. P. (2000). Optimism, pessimism, and mortality. Mayo Clinic Proceedings, 75, 133-134. doi: 10.4065/75.2.133

Seligman, M. E. P., Steen, T. A., Park, N., \& Peterson, C. (2005). Positive psychology progress: empirical validation of interventions. The American Psychologist, 60, 410-421. doi: 10.1037/0003-066X.60.5.410

Serin, R. (2007). The Dynamic Risk Assessment Scale for Offender Re-Entry (DRAOR). Unpublished scale. Ottawa, ON: Carleton University.

Sheldon, K. M., \& King, L. (2001). Why positive psychology is necessary. American Psychologist, 56, 216-217. doi: 10.1037/0003-066x.56.3.216

Shepherd, S. M., Adams, Y., McEntyre, E., \& Walker, R. (2014). Violence risk assessment in Australian Indigenous offender populations: A review of the literature. Psychology, Public Policy, and Law, 20, 281-293. doi: 10.1037/law0000017

Shepherd, S. M., Delgado, R. H., Sivasubramaniam, D., \& Paradies, Y. (2018). Predictors of distress and the protective impact of cultural engagement for Indigenous prisoners. Journal of Offender Rehabilitation, 57(6), 367-383. doi: $10.1080 / 10509674.2018 .1510867$

Simkins, S., \& Katz, S. (2002). Criminalizing abused girls. Violence Against Women, 8, 1474-1499. doi: $10.1177 / 107780102237966$

Simourd, D. J. (2004). Use of Dynamic Risk/Need Assessment Instruments Among Long-Term Incarcerated Offenders. Criminal Justice and Behavior, 31, 306-323. doi: $10.1177 / 0093854803262507$ 
Simourd, L., \& Andrews, D. A. (1994). Correlates of delinquency: A look at gender differences. Forum on Corrections Research, 6(1), 26-31. Retrieved December 1, 2013 from http://www.csc-scc.gc.ca/text/pblct/forum/e061/061g_e.pdf

Simourd, D., Hoge, R. D., Andrews, D. A. \& Leschied, A. W. (1994). An empiricallybased typology of youthful offenders. Canadian Journal of Criminology, 36, 447461.

Simpson, S. S., Yahner, J. L., \& Dugan, L. (2008). Understanding women’s pathways to jail: analysing the lives of incarcerated women. Australian and New Zealand Journal of Criminology, 41, 84-108. doi: 10.1375/acri.41.1.84.

Singer, J. D., \& Willett, J. B. (2003). Applied longitudinal data analysis: Modeling change and event occurrence. New York: Oxford University Press.

Smith, P., Cullen, F. T., \& Latessa, E. J. (2009). Can 14,737 women be wrong? A metaanalysis of the LSI-R and recidivism for female offenders. Criminology \& Public Policy, 8(1), 183-208. doi: 10.1111/j.1745-9133.2009.00551.x

Snyder, C. R., Lopez, S. J., \& Pedrotti, J. T. (2011). Positive psychology: The scientific and practical explorations of human strengths (2nd ed.). Sage Publications, Inc.

Stewart, L. A., Wardrop, K., Wilton, G., Thompson, J., Derkzen, D., \& Motiuk, L. (2017). Reliability and Validity of the Dynamic Factors Identification and Analysis-Revised (Research Report R-395). Ottawa, ON: Correctional Service Canada.

Steyerberg, E. W., Eijkemans, M. J. C., Harrell Jr, F. E., \& Habbema, J. D. F. (2001). Prognostic modeling with logistic regression analysis: In search of a sensible 
strategy in small data sets. Medical Decision Making, 21(1), 45-56. doi:

10.1177/0272989X0102100106

Stübner, S., Groß, G., \& Nedopil, N. (2006). Inpatient risk management with mentally ill offenders: Results of a survey on clinical decision-making about easing restrictions. Criminal Behaviour and Mental Health, 16, 111-123. doi: 10.1002/cbm.619

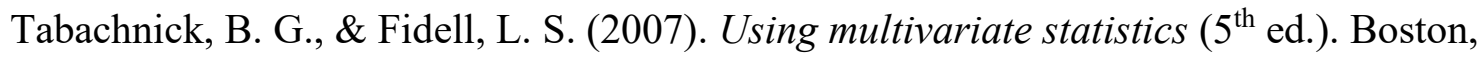
MA: Allyn and Bacon.

Tabachnick, B. G., \& Fidell, L. S. (2013). Using multivariate statistics $\left(6^{\text {th }}\right.$ ed.). Boston, MA: Pearson Education.

Vandenberg, R. J., \& Lance, C. E. (2000). A review and synthesis of the measurement invariance literature: Suggestions, practices, and recommendations for organizational research. Organizational Research Methods, 3(1), 4-70. doi: $10.1177 / 109442810031002$

Vanderbilt-Adriance, E., \& Shaw, D. S. (2008). Protective factors and the development of resilience in the context of neighborhood disadvantage. Journal of Abnormal Child Psychology, 36(6), 887-901. doi: 10.1007/s10802-008-9220-1

Van Voorhis, P. (2012). On behalf of women offenders: Women's place in the science of evidence-based practice. Criminology and Public Policy, 11, 111-145. doi: 10.1111/j.1745-9133.2012.00793.x

Van Voorhis, P., Wright, E. M., Salisbury, E., \& Bauman, A. (2010). Women's risk factors and their contributions to existing risk/needs assessment. Criminal Justice and Behavior, 37, 261-288. doi: 10.1177/0093854809357442 
Vermunt, J. K., \& Magidson, J. (2002). Latent class cluster analysis. In J. A. Hagenaars \& A. L. McCutcheon (eds.), Advances in Latent Class Analysis. Cambridge University Press.

Wanamaker, K. A., Jones, N. J., \& Brown, S. L. (2018). Strengths-based assessments for use with forensic populations: A critical review. International Journal of Forensic Mental Health, 17, 202-221. doi: 10.1080/14999013.2018.1451414

Wanamaker, K. A., Brown, S. L., \& Czerwinsky, A. (2020). The Prevalence and nature of victimization within justice-involved samples: A meta-analysis of gender differences and similarities (Submitted Manuscript).

Ward, T. (2002). The management of risk and the design of good lives. Australian Psychologist, 37, 172-179. doi: 10.1080/00050060210001706846

Ward, T. (2017). Prediction and agency: The role of protective factors in correctional rehabilitation and desistance. Aggression and Violent Behavior, 32, 19-28. doi: 10.1016/j.avb.2016.11.012

Ward, T., \& Brown, M. (2004). The good lives model and conceptual issues in offender rehabilitation. Psychology, Crime, \& Law, 10, 243-257. doi: $10.1080 / 10683160410001662744$

Ward, T., Mann, R. E., \& Gannon, T. A. (2007). The good lives model of offender rehabi litation: Clinical implications. Aggression and Violent Behavior, 12, 87-107. doi: 10.1016/j.avb.2006.03.004

Ward, T., \& Maruna, S. (2007). Rehabilitation: Beyond the risk assessment paradigm. London, UK: Routledge. 
Ward, T., \& Stewart, C. A. (2003). The treatment of sex offenders: Risk management and good lives. Professional Psychology: Research and Practice, 34, 353-360. doi: $10.1037 / 0735-7028.34 .4 .353$

Wardrop, K., Wanamaker, K. A., \& Derkzen, D. (2019). Developing a risk/need assessment tool for women offenders: a gender-informed approach. Journal of Criminological Research, Policy and Practice. doi: 10.1108/ JCRPP-03-20190017

Wattanaporn, K. A., \& Holtfreter, K. (2014). The Impact of Feminist Pathways Research on Gender-Responsive Policy and Practice. Feminist Criminology, 9(3), 191-207. doi: $10.1177 / 1557085113519491$

Werner, E. E. (1989). High-risk children in young adulthood: A longitudinal study from birth to 32 years. American Journal of Orthopsychiatry, 59, 72-81. doi : 10.1111/j.1939-0025.1989.tb01636.x

Werner, E. (2005). Resilience and recovery: Findings from the Kauai longitudinal study. Research, Policy, and Practice in Children's Mental Health, 19, 11-14.

Werner, E. E. \& R. S. Smith, (1977). Kauai's Children Come of Age. Honolulu: University of Hawaii Press.

Werner, E. E., \& Smith, R. S. (1982). Vulnerable but invincible: A longitudinal study of resilient children and youth. New York: McGraw Hill.

Werner, E. E., \& Smith, R. S. (1992). Overcoming the odds: High risk children from birth to adulthood. Ithaca, NY, US: Cornell University Press.

Wilson, H. A., \& Gutierrez, L. (2014). Does one size fit all?: A meta-analysis examining the predictive ability of the Level of Service Inventory (LSI) with Indigenous 
offenders. Criminal Justice and Behavior, 41, 196-219. doi:

$10.1177 / 0093854813500958$

Wilson, J. A., Naro, W., \& Austin, J. F. (2007). Innovations in probation: Assessing New York City's Automated Reporting System. Washington, DC: The JFA Institute. Retrieved from www.pbpp.pa.gov/Information/Documents/Research/OSR3.pdf

Wong, S. C. P., Olver, M. E., \& Stockdale, K. C. (2009). The utility of dynamic and static factors in risk assessment, prediction, and treatment. In J. T. Andrade (Ed.). Handbook of violence risk assessment and treatment: New approaches for mental health professionals (pp. 83-120). New York: Springer.

Woods, J. B. (2017). LGBT Identity and Crime. California Law Review, 105, 667-739. doi: 10.15779/Z389W08Z24

Wormith, S. J., Ferguson, M., \& Bonta, J. (2013). Offender classification and case management and their application in Canadian Corrections. In J. Winterdyck \& M. Z. Weinrath (Eds.), Adult corrections in Canada (pp. 171-198). Whitby: de Sitter Publications.

Yang, M., Guo, B., Olver, M. E., Polaschek, D. L. L., \& Wong, S. C. P. (2017). Assessing associations between changes in risk and subsequent reoffending. An introduction to relevant statistical models. Criminal Justice and Behavior, 44(1), 59-84. doi: $10.1177 / 0093854816678648$

Yates, T. M., \& Masten, A. S. (2004). Fostering the future: Resilience theory and the practice of positive psychology. In P. A. Linley \& S. Joseph (Eds.), Positive psychology in practice (pp. 521-539). Hoboken, NJ: Wiley. 
Zamble, E., \& Quinsey, V. L. (1997). The process of criminal recidivism. Cambridge: Cambridge University Press. 


\section{Appendix A}

\section{Detailed Description of the Service Planning Instrument}

(SPIn; Orbis Partners, 2003)

Table 1A

Description of SPIn Static and Dynamic Domains

\begin{tabular}{llll}
\hline Domain & Content $^{\mathrm{b}}$ & Description & Score range (Number of items) \\
\hline $\begin{array}{l}\text { Criminal } \\
\text { History }^{\mathrm{a}}\end{array}$ & $\begin{array}{l}\text { Static risk } \\
\text { Pre-Screen items }\end{array}$ & $\begin{array}{l}\text { Assesses past offences, youth dispositions, } \\
\text { previous adult convictions, age at first arrest, and } \\
\text { past incarcerations }\end{array}$ & Static risk: 0-20 (6 items) \\
$\begin{array}{l}\text { Response to } \\
\text { Supervision }\end{array}$ & $\begin{array}{l}\text { Static risk } \\
\text { Pre-Screen items }\end{array}$ & $\begin{array}{l}\text { Assesses breaches, technical violations, new } \\
\text { offences on supervision, escapes, disciplinary } \\
\text { infractions, and segregation }\end{array}$ & Static risk: 0-43 (10 items) \\
$\begin{array}{l}\text { Aggression/ } \\
\text { Violence }\end{array}$ & $\begin{array}{l}\text { Static risk } \\
\text { Dynamic risk } \\
\text { Dynamic strength } \\
\text { 5 Pre-Screen items }\end{array}$ & $\begin{array}{l}\text { Assesses previous violent convictions, violent } \\
\text { behaviour, weapon offences, threatening } \\
\text { behaviour, anger/frustration, opinions and beliefs } \\
\text { about physical and verbal aggression, and } \\
\text { frequency of conflicts }\end{array}$ & $\begin{array}{l}\text { Static risk: 0-41 (10 items) } \\
\text { Dynamic risk: 0-8 (4 items) }\end{array}$ \\
& & & \\
\hline
\end{tabular}




\begin{tabular}{|c|c|c|c|}
\hline Substance Use & $\begin{array}{l}\text { Static risk } \\
\text { Dynamic risk } \\
1 \text { Pre-Screen item }\end{array}$ & $\begin{array}{l}\text { Assesses types and number of times using } \\
\text { various drugs and alcohol, age at first use, } \\
\text { previous treatment, and motivation for seeking } \\
\text { treatment }\end{array}$ & 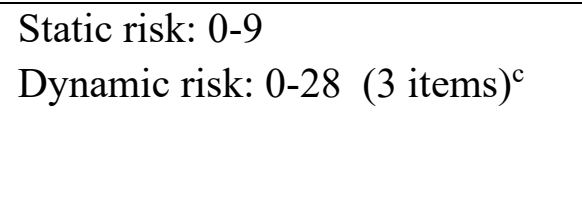 \\
\hline $\begin{array}{l}\text { Social } \\
\text { influences }\end{array}$ & $\begin{array}{l}\text { Dynamic risk } \\
\text { Dynamic strength } \\
2 \text { Pre-Screen items }\end{array}$ & $\begin{array}{l}\text { Assesses gang associations, peer relationships, } \\
\text { anti-social peers, social activity, community } \\
\text { engagement, and neighborhood influences }\end{array}$ & $\begin{array}{l}\text { Dynamic risk: } 0-26 \text { ( } 6 \text { items }) \\
\text { Dynamic strength: } 0-15 \text { ( } 5 \text { items) }\end{array}$ \\
\hline Family & $\begin{array}{l}\text { Static risk } \\
\text { Dynamic risk } \\
\text { Static strength } \\
\text { Dynamic strength } \\
3 \text { Pre-Screen items }\end{array}$ & $\begin{array}{l}\text { Assesses current intimate relationships, marital } \\
\text { risk factors, attachment to children, parenting, } \\
\text { family history, family involvement, pro-social } \\
\text { family models, and current relationships with } \\
\text { family }\end{array}$ & $\begin{array}{l}\text { Static risk: } 0-9 \text { (3 items) } \\
\text { Dynamic risk: } 0-26 \text { ( } 7 \text { items) } \\
\text { Static strength: } 0-4 \text { ( } 2 \text { items }) \\
\text { Dynamic strength: } 0-14 \text { ( } 7 \text { items) }\end{array}$ \\
\hline Employment & $\begin{array}{l}\text { Static risk } \\
\text { Dynamic risk } \\
\text { Static strength } \\
\text { Dynamic strength } \\
2 \text { Pre-Screen items }\end{array}$ & $\begin{array}{l}\text { Assesses employment history, education, } \\
\text { employment plans and performance, } \\
\text { marketability, and job search skills }\end{array}$ & $\begin{array}{l}\text { Static risk: } 0-9 \text { (2 items) } \\
\text { Dynamic risk: } 0-14 \text { (6 items) } \\
\text { Static strength: } 0-3 \text { ( } 1 \text { item) } \\
\text { Dynamic strength: } 0-12 \text { (5 items) }\end{array}$ \\
\hline Attitudes & $\begin{array}{l}\text { Dynamic risk } \\
\text { Dynamic strength } \\
2 \text { Pre-Screen items }\end{array}$ & $\begin{array}{l}\text { Assesses law-abiding attitudes, ability to accept } \\
\text { responsibility, commitment to criminal lifestyle, } \\
\text { attitude towards crime and the criminal justice } \\
\text { system, change readiness, and will to make } \\
\text { amends }\end{array}$ & $\begin{array}{l}\text { Dynamic risk: } 0-14 \text { (9 items) } \\
\text { Dynamic strength: } 0-14 \text { ( } 9 \text { items) }\end{array}$ \\
\hline
\end{tabular}




\begin{tabular}{|c|c|c|c|}
\hline $\begin{array}{l}\text { Social/ } \\
\text { Cognitive } \\
\text { Skills }\end{array}$ & $\begin{array}{l}\text { Dynamic risk } \\
\text { Dynamic strength } \\
2 \text { Pre-Screen items }\end{array}$ & $\begin{array}{l}\text { Assesses impulsivity, hostility, consequential } \\
\text { thinking, social perspective taking, problem } \\
\text { solving, behaviour control, interpersonal skills } \\
\text { and goal setting }\end{array}$ & $\begin{array}{l}\text { Dynamic risk: } 0-18 \text { ( } 8 \text { items }) \\
\text { Dynamic strength: } 0-18 \text { (8 items) }\end{array}$ \\
\hline Stability & $\begin{array}{l}\text { Static risk } \\
\text { Dynamic risk } \\
\text { Dynamic strength } \\
2 \text { Pre-Screen items }\end{array}$ & $\begin{array}{l}\text { Assesses financial and accommodation, } \\
\text { transportation, and life skills }\end{array}$ & $\begin{array}{l}\text { Static risk: } 0-2 \text { ( } 1 \text { item) } \\
\text { Dynamic risk: } 0-13 \text { (4 items) } \\
\text { Dynamic strength: } 0-7 \text { (4 items) }\end{array}$ \\
\hline $\begin{array}{l}\text { Mental } \\
\text { Health }\end{array}$ & $\begin{array}{l}\text { Flag }^{\text {ad }} \\
\text { Pre-Screen items }\end{array}$ & $\begin{array}{l}\text { Assesses mental health conditions, homicidal } \\
\text { ideation, suicidal ideation, history of sexual } \\
\text { aggression, victimization, and self-injurious } \\
\text { behaviours }\end{array}$ & flag: $0-2$ (5 items $)$ \\
\hline
\end{tabular}


Table 2A

Sample Items from Each Dynamic Domain of the SPIn Full Assessment

\begin{tabular}{|c|c|c|c|}
\hline Dynamic Domain & Domain Scores & Sample Item (Question) & Item Responses. Scores \\
\hline $\begin{array}{l}\text { Aggression / } \\
\text { Violence: Risk }\end{array}$ & $\begin{array}{l}\text { Low }=1 \\
\text { Mod }=2-3 \\
\text { High }=4+\end{array}$ & $\begin{array}{l}\text { Believes in the use of } \\
\text { physical violence to solve } \\
\text { conflicts or arguments }\end{array}$ & $\begin{array}{l}0=\text { Believes violence is not justifiable } \\
1=\text { Believes violence is often justifiable } \\
2=\text { Believes violence is usually the only option for } \\
\text { solving conflict }\end{array}$ \\
\hline $\begin{array}{l}\text { Aggression / } \\
\text { Violence: Strength }\end{array}$ & $\begin{array}{l}\text { Low }=1-2 \\
\text { Mod }=3-4 \\
\text { High }=5+\end{array}$ & $\begin{array}{l}\text { Conflict with others } \\
\text { (including staff, peers, } \\
\text { family, etc.) }\end{array}$ & $\begin{array}{l}0=\text { Sometimes in conflict with others } \\
1=\text { Rarely in conflict with others } \\
2=\text { Never in conflict with others }\end{array}$ \\
\hline Substance use: Risk & $\begin{array}{l}\text { Low }=1-4 \\
\text { Mod }=5-17 \\
\text { High }=18+\end{array}$ & Cocaine/crack times used & $\begin{array}{l}0=\text { None } / \text { fewer than } 1 \text { time per week } \\
3=1-2 \text { times per week } \\
5=3-6 \text { times per week } \\
8=\text { Daily }\end{array}$ \\
\hline $\begin{array}{l}\text { Social Influences: } \\
\text { Risk }\end{array}$ & $\begin{array}{l}\text { Low }=1-2 \\
\text { Mod }=3-6 \\
\text { High }=7+\end{array}$ & Anti-social peers & $\begin{array}{l}0=\text { No/avoids anti-social peers and individuals } \\
1=\text { Associates with anti-social peers at lower risk of } \\
\text { frequent or serious offending } \\
2=\text { Associates with anti-social peers at high risk of } \\
\text { frequent or serious offending }\end{array}$ \\
\hline
\end{tabular}




\begin{tabular}{|c|c|c|c|}
\hline $\begin{array}{l}\text { Social Influences: } \\
\text { Strength }\end{array}$ & $\begin{array}{l}\text { Low }=1-4 \\
\text { Mod }=5-8 \\
\text { High }=9+\end{array}$ & Community participation & $\begin{array}{l}0=\text { No participation in pro-social community } \\
\text { organizations } \\
1=\text { Interest but no current participation in pro-social } \\
\text { community organizations } \\
2=\text { Participation in pro-social community } \\
\text { organizations } \\
3=\text { Highly involved in pro-social community } \\
\text { organizations }\end{array}$ \\
\hline Family: Risk & $\begin{array}{l}\text { Low }=1-2 \\
\text { Mod }=3-7 \\
\text { High }=8+\end{array}$ & $\begin{array}{l}\text { Current intimate } \\
\text { relationship }\end{array}$ & $\begin{array}{l}0=\text { Marital satisfaction/stability in relationship/no } \\
\text { relationship } \\
3=\text { Some conflict and dissatisfaction evident in the } \\
\text { relationship } \\
5=\text { High degree of instability and conflict; high } \\
\text { dissatisfaction }\end{array}$ \\
\hline Family: Strength & $\begin{array}{l}\text { Low }=1-3 \\
\text { Mod }=4-5 \\
\text { High }=6+\end{array}$ & Attachment to children & $\begin{array}{l}0=\text { High degree of conflict; absence of any positive } \\
\text { contact with children; lacks interest in developing } \\
\text { relationship with children; no children } \\
1=\text { Rewarding relationships with children } \\
2=\text { Highly rewarding relationship with major } \\
\text { expression of commitment to parenting role }\end{array}$ \\
\hline
\end{tabular}




\begin{tabular}{|c|c|c|c|}
\hline $\begin{array}{l}\text { Education and } \\
\text { Employment: Risk }\end{array}$ & $\begin{array}{l}\text { Low }=1-2 \\
\text { Mod }=3-6 \\
\text { High }=7+\end{array}$ & Employment performance & $\begin{array}{l}0=\text { Usually meets or exceeds employment } \\
\text { requirements } \\
1=\text { Some evidence of poor work habits } \\
2=\text { Clear evidence of poor work habits (e.g., } \\
\text { unreliable, tardy) }\end{array}$ \\
\hline $\begin{array}{l}\text { Education and } \\
\text { Employment: } \\
\text { Strength }\end{array}$ & $\begin{array}{l}\text { Low }=1-2 \\
\text { Mod }=3-7 \\
\text { High }=8+\end{array}$ & Employment motivation & $\begin{array}{l}0=\text { Expresses no interest or no motivation to find } \\
\text { employment; recognizes need for permanent } \\
\text { employment } \\
1 \text { = Expresses sincere desire to find and maintain } \\
\text { employment } \\
2=\text { Intrinsically motivated to find and maintain } \\
\text { employment; enjoys work }\end{array}$ \\
\hline Attitudes: Risk & $\begin{array}{l}\text { Low }=1-2 \\
\text { Mod }=3-5 \\
\text { High }=6+\end{array}$ & Law-abiding attitudes & $\begin{array}{l}0=\text { Expresses neutral attitude or desire to live in a } \\
\text { law-abiding manner; positive commitment towards } \\
\text { law-abiding behaviour } \\
1 \text { = Feels law-abiding behaviour does not apply to } \\
\text { him or her } \\
2=\text { Openly admits unwillingness to demonstrate law- } \\
\text { abiding behaviour }\end{array}$ \\
\hline
\end{tabular}




\begin{tabular}{|c|c|c|c|}
\hline Attitudes: Strength & $\begin{array}{l}\text { Low }=1-5 \\
\text { Mod }=6-10 \\
\text { High }=11+\end{array}$ & Accepts responsibility & $\begin{array}{l}0=\text { accepts and is proud of criminal behaviour; } \\
\text { minimizes, denies, justifies; blames others; indicates } \\
\text { some awareness } \\
1=\text { Recognizes he/she must accept responsibility } \\
2=\text { Voluntarily accepts full responsibility for crime }\end{array}$ \\
\hline $\begin{array}{l}\text { Social/Cognitive: } \\
\text { Risk }\end{array}$ & $\begin{array}{l}\text { Low }=1-2 \\
\text { Mod }=3-4 \\
\text { High }=5+\end{array}$ & Impulsivity & $\begin{array}{l}0=\text { Can identify is aware of triggers/ self-control } \\
\text { techniques } \\
1=\text { Usually fails to identify triggers } \\
2=\text { Cannot identify triggers that cause problem } \\
\text { behaviours }\end{array}$ \\
\hline $\begin{array}{l}\text { Social/Cognitive: } \\
\text { Strength }\end{array}$ & $\begin{array}{l}\text { Low }=1-3 \\
\text { Mod }=4-9 \\
\text { High }=10+\end{array}$ & Problem-solving & $\begin{array}{l}0=\text { Cannot identify problems and/or generate } \\
\text { solutions } \\
1=\text { Can generate different solutions to problems } \\
2=\text { Can successfully apply appropriate solutions to } \\
\text { problems }\end{array}$ \\
\hline Stability: Risk & $\begin{array}{l}\text { Low }=1-2 \\
\text { Mod }=3-5 \\
\text { High }=6+\end{array}$ & Transportation & $\begin{array}{l}0=\text { Reliable access to/ funds available for } \\
\text { transportation } \\
1=\text { No license; unreliable access to needed } \\
\text { transportation } \\
2=\text { Lack of access to needed transportation }\end{array}$ \\
\hline
\end{tabular}




\begin{tabular}{|c|c|c|c|}
\hline Stability: Strength & $\begin{array}{l}\text { Low }=1-3 \\
\text { Mod }=4 \\
\text { High }=5+\end{array}$ & Financial & $\begin{array}{l}0=\text { Social assistance; low income; high debt; no } \\
\text { insurance } \\
1=\text { income reasonably adequate } \\
2=\text { Comfortable financial situation }\end{array}$ \\
\hline
\end{tabular}

Note. Mod $=$ moderate. 


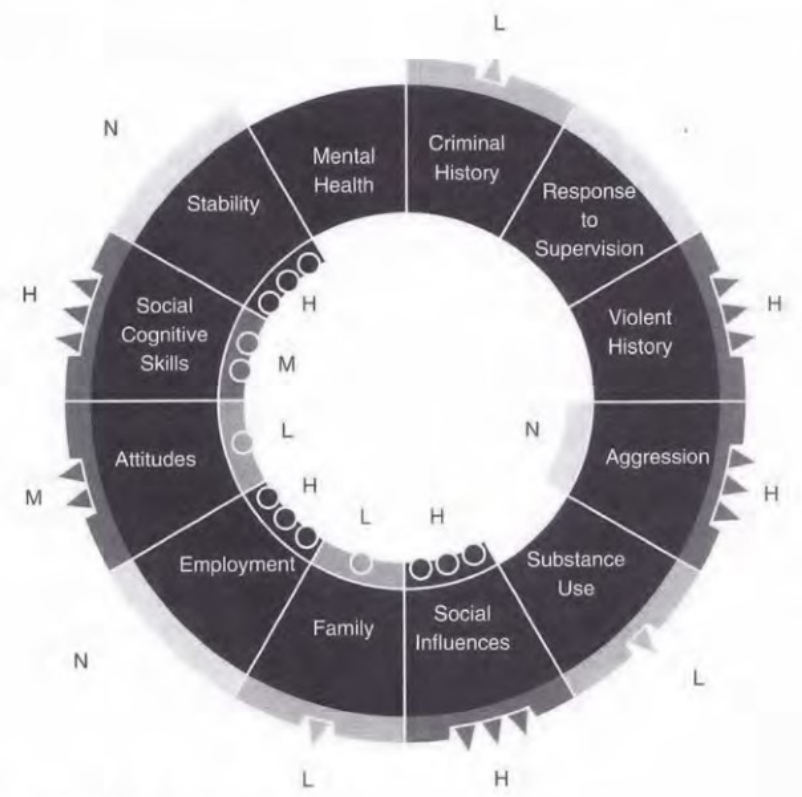

Figure 1A. Example of SPIn wheel (from Jones \& Robinson, 2018). 


\section{Appendix B}

\section{Internal Consistency of the Dynamic Risk and Strength Domains}

\section{Table 1B}

Internal consistency of the domains for men, women and total sample

\begin{tabular}{|c|c|c|c|c|}
\hline \multirow[b]{2}{*}{ SPIn Dynamic Domains } & \multirow[b]{2}{*}{ \# of items } & Overall & Men & Women \\
\hline & & \multicolumn{3}{|c|}{$\alpha$} \\
\hline Aggression/Violence Risk & 4 & .85 & .86 & .84 \\
\hline Aggression/Violence Strength & 4 & .89 & .90 & .88 \\
\hline Substance Use Risk & $3^{\mathrm{a}}$ & .77 & .76 & .79 \\
\hline Social Influences Risk & 6 & .67 & .68 & .65 \\
\hline Social Influences Strength & 5 & .59 & .59 & .60 \\
\hline Family Risk & 7 & .48 & .47 & .50 \\
\hline Family Strength & 7 & .68 & .69 & .68 \\
\hline Employment Risk & 6 & .79 & .80 & .78 \\
\hline Employment Strength & 5 & .80 & .81 & .80 \\
\hline Attitudes Risk & 9 & .82 & .82 & .81 \\
\hline Attitudes Strength & 9 & .89 & .89 & .88 \\
\hline Skills Risk & 8 & .86 & .87 & .86 \\
\hline Skills Strength & 8 & .91 & .91 & .92 \\
\hline Stability Risk & 4 & .57 & .58 & .55 \\
\hline Stability Strength & 4 & .60 & .60 & .59 \\
\hline
\end{tabular}

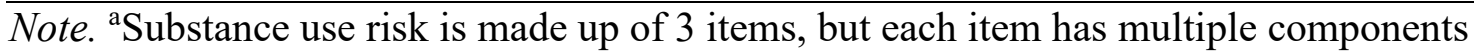
that are not used to calculate alpha, as they are only assessed if relevant and are used for case planning. These items include types of drugs and frequency of use. $\alpha=$ Cronbach's alpha. 


\section{Appendix C}

\section{Analyses for Study 1}

Table 1C

Breakdown of Analyses for Study 1

\begin{tabular}{|c|c|c|}
\hline Analyses & Statistical Method & Software \\
\hline Descriptives & Frequencies & SPSS \\
\hline \multirow[t]{3}{*}{ Basic Data Cleaning } & T-test/ANOVA & \\
\hline & Chi-square & \\
\hline & Correlations & \\
\hline \multirow[t]{7}{*}{ Measurement Invariance } & Step 1: Select sample & Mplus \\
\hline & $\begin{array}{l}\text { Step 2: Statistical assumption } \\
\text { checking specific to EFA }\end{array}$ & \\
\hline & Step 3: Initial Exploratory Factor & \\
\hline & Analysis to determine rotation type & \\
\hline & $\begin{array}{l}\text { Step 4: Exploratory Factor Analysis } \\
\text { across three timepoints }\end{array}$ & \\
\hline & Step 5: Exploratory Structural & \\
\hline & Equation Modeling & \\
\hline Patterns of Change & Multilevel Growth Modeling & SAS \\
\hline \multirow[t]{3}{*}{ Change and Recidivism } & Two-stage Multilevel Growth & \\
\hline & Modeling (extract change parameters) & \\
\hline & Logistic Regression & \\
\hline
\end{tabular}




\section{Appendix D}

Means, Standard Deviations, and Correlations between SPIn Dynamic Domains for Each of the Three Timepoints used for Measurement Invariance for Each Gender

Table 1D

Dynamic Domain Score Means and Standard Deviations for Men across Timepoints

\begin{tabular}{lccc}
\hline & Time $1_{(\text {(initial })}$ & Time $2_{(3-8 \text { months })}$ & Time $3(9-14$ months $)$ \\
\cline { 2 - 4 } SPIn Dynamic Domains & & $M(S D)$ & \\
\hline Aggression/Violence Risk & $0.40(1.12)$ & $0.36(1.06)$ & $0.34(1.03)$ \\
Aggression/Violence Strength & $1.84(2.00)$ & $1.96(2.04)$ & $2.02(2.05)$ \\
Substance Use Risk & $6.63(7.90)$ & $6.00(7.02)$ & $5.69(6.61)$ \\
Social Influences Risk & $3.01(3.53)$ & $2.94(3.49)$ & $2.92(3.50)$ \\
Social Influences Strength & $4.50(3.32)$ & $4.66(3.36)$ & $4.73(3.38)$ \\
Family Risk & $4.58(4.26)$ & $4.60(4.21)$ & $4.62(4.20)$ \\
Family Strength & $3.57(2.75)$ & $3.68(2.79)$ & $3.74(2.82)$ \\
Employment Risk & $0.69(1.74)$ & $0.69(1.70)$ & $0.71(1.74)$ \\
Employment Strength & $3.46(2.96)$ & $3.61(3.03)$ & $3.70(3.07)$ \\
Attitudes Risk & $1.36(2.28)$ & $1.34(2.30)$ & $1.33(2.30)$ \\
Attitudes Strength & $5.38(4.33)$ & $5.61(4.44)$ & $5.70(4.50)$ \\
Skills Risk & $1.00(2.08)$ & $0.96(2.07)$ & $0.94(2.05)$ \\
Skills Strength & $3.64(3.73)$ & $3.86(3.86)$ & $3.98(3.94)$ \\
Stability Risk & $1.48(2.01)$ & $1.46(2.01)$ & $1.45(2.01)$ \\
Stability Strength & $3.94(1.69)$ & $4.03(1.68)$ & $4.07(1.69)$ \\
\hline Note. & & & \\
\hline
\end{tabular}

Note. $M=$ Mean. $S D=$ Standard deviation. 
Table 2D

Dynamic Domain Means and Standard Deviations for Women across Timepoints

\begin{tabular}{lccc}
\hline & Time $1_{\text {(initial) }}$ & Time $2(3-8$ months) & Time $3(9-14$ months $)$ \\
\cline { 2 - 4 } SPIn Dynamic Domains & $0.28(.89)$ & $0.25(.85)$ & $0.25(.86)$ \\
\hline Aggression/Violence Risk & $1.99(2.14)$ & $2.10(2.18)$ & $2.16(2.20)$ \\
Aggression/Violence Strength & $6.23(8.19)$ & $5.57(7.00)$ & $5.36(6.71)$ \\
Substance Use Risk & $2.83(3.43)$ & $2.79(3.45)$ & $2.78(3.47)$ \\
Social Influences Risk & $4.43(3.25)$ & $4.59(3.30)$ & $4.66(3.33)$ \\
Social Influences Strength & $4.48(4.26)$ & $4.52(4.26)$ & $4.54(4.22)$ \\
Family Risk & $3.65(2.77)$ & $3.76(2.80)$ & $3.81(2.80)$ \\
Family Strength & $1.24(2.29)$ & $1.26(2.33)$ & $1.30(2.37)$ \\
Employment Risk & $2.31(2.55)$ & $2.38(2.59)$ & $2.42(2.65)$ \\
Employment Strength & $0.90(1.73)$ & $0.88(1.72)$ & $0.89(1.77)$ \\
Attitudes Risk & $6.14(4.58)$ & $6.35(4.70)$ & $6.48(4.75)$ \\
Attitudes Strength & $0.73(1.69)$ & $0.66(1.65)$ & $0.68(1.67)$ \\
Skills Risk & $3.91(3.92)$ & $4.17(4.06)$ & $4.27(4.13)$ \\
Skills Strength & $1.92(2.16)$ & $1.95(2.19)$ & $1.96(2.23)$ \\
Stability Risk & $3.50(1.60)$ & $3.55(1.60)$ & $3.60(1.61)$ \\
Stability Strength & & &
\end{tabular}

Note. $M=$ Mean. $S D=$ Standard deviation. 
Table 3D

Correlations between Dynamic Domain Scores by Gender for Time 1 (Initial Assessment, within 90 days of Supervision Start)

\begin{tabular}{|c|c|c|c|c|c|c|c|c|c|c|c|c|c|c|c|}
\hline Domain & $\begin{array}{r}\text { AV: } \\
\text { DR }\end{array}$ & $\begin{array}{r}\text { AV: } \\
\text { DS }\end{array}$ & $\begin{array}{l}\text { SU: } \\
\text { DR }\end{array}$ & $\begin{array}{l}\text { SI: } \\
\text { DR }\end{array}$ & $\begin{array}{l}\text { SI: } \\
\text { DS }\end{array}$ & $\begin{array}{r}\text { FM: } \\
\text { DR }\end{array}$ & $\begin{array}{r}\text { FM: } \\
\text { DS }\end{array}$ & $\begin{array}{l}\text { EE: } \\
\text { DR }\end{array}$ & $\begin{array}{r}\text { EE: } \\
\text { DS }\end{array}$ & $\begin{array}{r}\text { AT: } \\
\text { DR }\end{array}$ & $\begin{array}{r}\text { AT: } \\
\text { DS }\end{array}$ & $\begin{array}{l}\text { SK: } \\
\text { DR }\end{array}$ & $\begin{array}{r}\text { SK: } \\
\text { DS }\end{array}$ & $\begin{array}{l}\text { ST: } \\
\text { DR }\end{array}$ & $\begin{array}{l}\text { ST: } \\
\text { DS }\end{array}$ \\
\hline AV: DR & 1 & -.217 & .146 & .227 & .083 & .295 & -.091 & .221 & -.043 & .388 & -.112 & .566 & -.177 & .220 & -.171 \\
\hline AV: DS & 281 & 1 & .055 & .054 & .413 & -.062 & .442 & .014 & .360 & -.085 & .631 & -.124 & .605 & .086 & .331 \\
\hline SU: DR & .147 & .012 & 1 & .367 & -.082 & .174 & -.034 & .229 & -.031 & .122 & .067 & .181 & -.027 & .249 & -.060 \\
\hline SI: DR & .313 & -.052 & .297 & 1 & -.318 & .347 & -.109 & .397 & -.133 & .363 & -.074 & .372 & -.138 & .488 & -.217 \\
\hline S & -.161 & .412 & -.083 & -.324 & 1 & -.107 & .467 & -.171 & .454 & -.142 & .511 & -.158 & .522 & -.169 & .462 \\
\hline FM & .294 & -.169 & .096 & .172 & -.109 & 1 & -.219 & .250 & -.065 & .292 & -.006 & .264 & -.090 & .310 & -.137 \\
\hline & & .404 & 4 &.-( & .439 & -.185 & 1 & -.120 & .377 & 9 & 501 & 56 & .509 & -.113 & .421 \\
\hline $\mathrm{EH}$ & .273 & -.094 & .177 & .349 & -.203 & .106 & -.144 & 1 & -.351 & .306 & 36 & .435 & -.197 & .492 & -.260 \\
\hline $\mathrm{E}$ & -.068 & .381 & -.018 & -.100 & .427 & .005 & .431 & -.332 & 1 & 099 & .476 & -.169 & .536 & -.223 & .459 \\
\hline AT & .517 & -.192 & .121 & .375 & -.167 & .293 & -.093 & .287 & -.069 & 1 & -.330 & .551 & -.219 & .295 & -.132 \\
\hline AT: DS & -.213 & .595 & .012 & -.152 & .478 & -.121 & .437 & -.141 & .471 & -.417 & 1 & -.220 & .719 & -.009 & .381 \\
\hline SK: DR & .594 & -.212 & .156 & .365 & -.209 & .252 & -.151 & .440 & -.160 & .634 & -.302 & 1 & -.312 & .370 & -.186 \\
\hline SK: DS & -.229 & .611 & -.043 & -.152 & .489 & -.163 & .450 & -.204 & .504 & -.261 & .703 & -.359 & 1 & -.119 & .441 \\
\hline ST: DR & .220 & -.049 & .248 & .392 & -.199 & .222 & -.129 & .476 & -.219 & .248 & -.086 & .344 & -.169 & 1 & -.519 \\
\hline ST: DS & -.089 & .319 & -.075 & -.150 & .417 & -.067 & .407 & -.314 & .493 & -.055 & .320 & -.174 & .396 & -.565 & 1 \\
\hline
\end{tabular}

Notes. AV:DR = Aggression/violence dynamic risk; AV: DS = Aggression/violence dynamic strength; SU:DR = Substance use dynamic risk; SI: DR = Social influences dynamic risk; SI: DS = Social influences dynamic strength; FM: DR = Family dynamic risk; FM: DS = Family dynamic strength; EE: DR = Employment dynamic risk; EE: DS = Employment dynamic strength; AT: DR = Attitudes dynamic risk; AT: DS = Attitudes dynamic strength; SK: DR = Skills dynamic risk; SK: DS = Skills dynamic strength; ST: $\mathrm{DR}=$ Stability dynamic risk; ST: DS = Stability dynamic strength. ${ }^{*}$ Bold correlations $=$ men. 
Table 4D

Correlations between Dynamic Domain Scores by Gender for Time 2 (3 to 8 Months Post Initial Assessment)

\begin{tabular}{|c|c|c|c|c|c|c|c|c|c|c|c|c|c|c|c|}
\hline & $\begin{array}{r}\text { AV: } \\
\text { DR } \\
\end{array}$ & $\begin{array}{r}\text { AV: } \\
\text { DS }\end{array}$ & $\begin{array}{l}\text { SU: } \\
\text { DR }\end{array}$ & $\begin{array}{l}\text { SI: } \\
\text { DR } \\
\end{array}$ & $\begin{array}{l}\text { SI: } \\
\text { DS }\end{array}$ & $\begin{array}{r}\text { FM: } \\
\text { DR }\end{array}$ & $\begin{array}{r}\text { FM: } \\
\text { DS }\end{array}$ & $\begin{array}{l}\text { EE: } \\
\text { DR }\end{array}$ & $\begin{array}{r}\text { EE: } \\
\text { DS }\end{array}$ & $\begin{array}{l}\text { AT: } \\
\text { DR } \\
\end{array}$ & $\begin{array}{r}\text { AT: } \\
\text { DS } \\
\end{array}$ & $\begin{array}{l}\text { SK: } \\
\text { DR }\end{array}$ & $\begin{array}{r}\text { SK: } \\
\text { DS }\end{array}$ & $\begin{array}{l}\text { ST: } \\
\text { DR }\end{array}$ & $\begin{array}{l}\text { ST: } \\
\text { DS }\end{array}$ \\
\hline AV: DR & 1 & 223 & .128 & .216 & .095 & .270 & .100 & .218 & -.078 & .333 & 126 & .533 & -.187 & .195 & -.095 \\
\hline AV: DS & 282 & 1 & .059 & .028 & .433 & -.064 & .449 & -.006 & .375 & -.085 & .636 & 135 & .606 & .038 & .342 \\
\hline SL & 131 & .019 & 1 & .372 & -.101 & .167 & 032 & .254 & -.040 & .153 & .055 & .173 & -.026 & .257 & -.080 \\
\hline SI: DR & .309 & 061 & .287 & 1 & -.333 & .356 & -.132 & .403 & -.163 & .386 & -.110 & .371 & -.157 & .493 & -.237 \\
\hline SI: DS & .151 & .420 & 070 & -.335 & 1 & -.119 & .477 & -.174 & .459 & -.159 & .521 & -.163 & .536 & -.176 & .460 \\
\hline FM: DR & . 299 & 173 & .090 & .180 & -.105 & 1 & -.223 & .260 & -.093 & .259 & -.024 & .242 & -.094 & .322 & -.159 \\
\hline FM: DS & -.109 & .415 & -.001 & -.069 & .435 & -.191 & 1 & -.133 & .378 & -.090 & .507 & -.156 & .508 & -.137 & .436 \\
\hline $\mathrm{EI}$ & .263 & -.095 & .174 & .361 & -.197 & .118 & -.142 & 1 & -.364 & .337 & -.104 & .419 & -.187 & .518 & -.295 \\
\hline EE: DS & -.077 & .396 & -.012 & -.111 & .425 & -.006 & .424 & -.338 & 1 & -.132 & .470 & -.171 & .526 & -.265 & .491 \\
\hline AT: DR & .533 & .199 & .118 & .386 & -.176 & .291 & -.109 & .298 & -.082 & 1 & -.334 & .501 & -.214 & .317 & -.155 \\
\hline AT: DS & -.228 & .604 & .034 & -.163 & .484 & -.124 & .445 & -.138 & .461 & -.420 & 1 & -.227 & .732 & -.045 & .404 \\
\hline SK: DR & .597 & -.217 & .148 & .360 & -.204 & .252 & -.161 & .442 & -.170 & .635 & -.304 & 1 & -.307 & .367 & -.213 \\
\hline SK: DS & -.237 & .619 & -.031 & -.161 & .494 & -.163 & .460 & -.201 & .502 & -.269 & .709 & -.362 & 1 & -.145 & .451 \\
\hline ST: DR & .235 & -.062 & .241 & .411 & -.200 & .230 & -.133 & .486 & -.236 & .268 & -.102 & .357 & -.181 & 1 & -.541 \\
\hline ST: DS & 122 & .337 & 080 & 177 & .425 & -.077 & .405 & -.327 & .501 & -.094 & .341 & -.193 & .409 & -.559 & 1 \\
\hline
\end{tabular}

Notes. AV:DR = Aggression/violence dynamic risk; AV: DS = Aggression/violence dynamic strength; SU:DR = Substance use dynamic risk; SI: DR = Social influences dynamic risk; SI: DS = Social influences dynamic strength; FM: DR = Family dynamic risk; FM: DS = Family dynamic strength; EE: DR = Employment dynamic risk; EE: DS = Employment dynamic strength; AT: DR = Attitudes dynamic risk; AT: DS = Attitudes dynamic strength; SK: DR = Skills dynamic risk; SK: DS = Skills dynamic strength; ST: $\mathrm{DR}=$ Stability dynamic risk; ST: DS = Stability dynamic strength. ${ }^{*}$ Bold correlations $=$ men. 
Table 5D

Correlations between Dynamic Domain Scores by Gender for Time 3 (9 to 14 Months Post Initial Assessment)

\begin{tabular}{|c|c|c|c|c|c|c|c|c|c|c|c|c|c|c|c|}
\hline & $\begin{array}{r}\text { AV: } \\
\text { DR }\end{array}$ & $\begin{array}{r}\text { AV: } \\
\text { DS }\end{array}$ & $\begin{array}{l}\text { SU: } \\
\text { DR }\end{array}$ & $\begin{array}{r}\text { SI: } \\
\text { DR }\end{array}$ & $\begin{array}{l}\text { SI: } \\
\text { DS }\end{array}$ & $\begin{array}{r}\text { FM: } \\
\text { DR }\end{array}$ & $\begin{array}{r}\text { FM: } \\
\text { DS }\end{array}$ & $\begin{array}{l}\text { EE: } \\
\text { DR }\end{array}$ & $\begin{array}{l}\text { EE: } \\
\text { DS }\end{array}$ & $\begin{array}{l}\text { AT: } \\
\text { DR }\end{array}$ & $\begin{array}{r}\text { AT: } \\
\text { DS }\end{array}$ & $\begin{array}{l}\text { SK: } \\
\text { DR }\end{array}$ & $\begin{array}{r}\text { SK: } \\
\text { DS }\end{array}$ & $\begin{array}{l}\text { ST: } \\
\text { DR }\end{array}$ & $\begin{array}{l}\text { ST: } \\
\text { DS }\end{array}$ \\
\hline & 1 & 23 & 130 & 23 & 098 & .262 & .115 & 250 & 1 & .342 & -.140 & .515 & .19 & 240 & .137 \\
\hline & 78 & 1 & 56 & .022 & 438 & 085 & .452 & 22 & .377 & 098 & .644 & 139 & .610 & 025 & 346 \\
\hline & .134 & 11 & 1 & .353 & 0 & .187 & -.045 & .25 & ( & .1 & .04 & .174 & -.028 & 253 & .070 \\
\hline & 12 & 070 & .284 & 1 & 337 & .364 & -.134 & .433 & -.177 & .424 & 125 & .384 & -.169 & .501 & -.246 \\
\hline S & TO & 24 & $07 / 5$ & 338 & 1 & -.123 & .477 & .100 & .461 & J & .529 & -.175 & .547 & ו & .463 \\
\hline FM: & 82 & 168 & .098 & .179 & 106 & 1 & -.224 & .272 & -.096 & .285 & -.030 & .264 & -.104 & .335 & -.163 \\
\hline $\mathrm{F}$ & -.111 & 17 & -.005 & ? & 1 & $10 ?$ & 1 & 27 & .374 & 93 & .504 & 7 & 507 & 146 & .424 \\
\hline & .267 & 105 & .177 & .378 & 197 & .134 & -.149 & 1 & -.370 & .386 & -.130 & .460 & -.209 & .534 & -.309 \\
\hline S & 93 & .403 & -.018 & -.122 & .420 & -.012 & .425 & -.348 & 1 & -.149 & .477 & -.185 & .542 & -.283 & .501 \\
\hline $\mathrm{A}^{-}$ & 36 & 202 & .125 & .393 & 76 & .280 & -.112 & .015 & -.090 & 1 & -.340 & .544 &.$- \angle \angle J$ & .338 & -.175 \\
\hline $\mathrm{A}^{\prime}$ & -.228 & 606 & .021 & -.179 & .489 & -.120 & .448 & -.146 & .460 & -.422 & 1 & -.236 & .723 & -.063 & .410 \\
\hline $\mathrm{SI}$ & 96 & 217 & .156 & 73 & 206 & .241 & -.164 & .454 & -.179 & .626 & 303 & 1 & -.31 & .40 & -.244 \\
\hline $\mathrm{SF}$ & -.232 & .620 & -.034 & -.169 & .494 & -.158 & .460 & -.208 & .503 & -.269 & .711 & -.362 & 1 & -.167 & .467 \\
\hline ST: DR & .235 & 068 & .246 & .414 & -.204 & .240 & -.145 & .495 & -.249 & .265 & -.110 & .360 & -.187 & 1 & -.548 \\
\hline ST: DS & 133 & .339 & 093 & .186 & .423 & -.081 & .406 & -.338 & .507 & -.102 & .344 & -.205 & .407 & -.564 & 1 \\
\hline
\end{tabular}

Notes. AV:DR = Aggression/violence dynamic risk; AV: DS = Aggression/violence dynamic strength; SU:DR = Substance use dynamic risk; SI: DR = Social influences dynamic risk; SI: DS = Social influences dynamic strength; FM: DR = Family dynamic risk; FM: DS = Family dynamic strength; EE: DR = Employment dynamic risk; EE: DS = Employment dynamic strength; AT: DR = Attitudes dynamic risk; AT: DS = Attitudes dynamic strength; SK: DR = Skills dynamic risk; SK: DS = Skills dynamic strength; ST: DR = Stability dynamic risk; ST: DS $=$ Stability dynamic strength. ${ }^{*}$ Bold correlations $=$ men. 


\section{Appendix E}

\section{Varimax Rotated Loadings and Standard Errors across Timepoints and For Each Gender}

Table 1E

Varimax Rotated Loadings and Standard Errors from EFAs across Three Timepoints for Men

\begin{tabular}{|c|c|c|c|c|c|c|c|c|c|}
\hline \multirow{2}{*}{$\begin{array}{l}\text { SPIn Dynamic } \\
\text { Domains }\end{array}$} & \multicolumn{3}{|c|}{ Time $1_{\text {(initial) }}$} & \multicolumn{3}{|c|}{ Time 2 (3-8 months) } & \multicolumn{3}{|c|}{ Time $3_{\text {(9-14 months) }}$} \\
\hline & F1 & F2 & F3 & F1 & F2 & F3 & F1 & F2 & F3 \\
\hline Aggression Risk & $-.13(.01)$ & $.66(.01)$ & $.08(.01)$ & $-.14(.01)$ & $.67(.01)$ & $.09(.01)$ & $-.14(.01)$ & $.66(.01)$ & $.10(.01)$ \\
\hline Aggression & $.71(.01)$ & $-.12(.01)$ & $-.03(.01)$ & $.72(.01)$ & $-.13(.01)$ & $-.03(.01)$ & $.72(.01)$ & $-.12(.01)$ & $-.04(.01)$ \\
\hline \multicolumn{10}{|l|}{ Strength } \\
\hline Substance use Risk & -- & -- & -- & -- & -- & -- & -- & -- & -- \\
\hline Social Risk & $-.04(.01)$ & $.44(.01)$ & $.41(.01)$ & $-.05(.01)$ & $.43(.01)$ & $.41(.01)$ & $-.06(.01)$ & $.44(.01)$ & $.41(.01)$ \\
\hline Social Strength & $.57(.01)$ & $-.09(.01)$ & $-.27(.01)$ & $.57(.01)$ & $-.08(.01)$ & $-.27(.01)$ & $.57(.01)$ & $-.08(.01)$ & $-.27(.01)$ \\
\hline Family Risk & -- & -- & -- & -- & -- & -- & -- & -- & -- \\
\hline Family Strength & $.57(.01)$ & $.02(.01)$ & $-.22(.01)$ & $.57(.01)$ & $.00(.01)$ & $-.20(.01)$ & $.57(.01)$ & $.00(.01)$ & $-.21(.01)$ \\
\hline Employ Risk & $-.08(.01)$ & $.36(.01)$ & $.49(.01)$ & $-.08(.01)$ & $.35(.01)$ & $.51(.01)$ & $-.08(.01)$ & $.37(.01)$ & $.52(.01)$ \\
\hline Employ Strength & $.59(.01)$ & $.05(.01)$ & $-.35(.01)$ & $.58(.01)$ & $.04(.01)$ & $-.36(.01)$ & $.57(.01)$ & $.04(.01)$ & $-.38(01)$ \\
\hline Attitudes Risk & $-.19(.01)$ & $.78(.01)$ & $.03(.01)$ & $-.19(.01)$ & $.78(.01)$ & $.05(.01)$ & $-.19(.01)$ & $.78(.01)$ & $.05(.01)$ \\
\hline Attitudes Strength & $.81(.01)$ & $-.25(.01)$ & $-.01(.01)$ & $.81(.01)$ & $-.26(.01)$ & $-.01(.01)$ & $.81(.01)$ & $-.26(.01)$ & $-.02(.01)$ \\
\hline Skills Risk & $-.18(.01)$ & $.77(.01)$ & $.20(.01)$ & $-.18(.01)$ & $.76(.01)$ & $.21(.01)$ & $-.18(.01)$ & $.75(.01)$ & $.22(.01)$ \\
\hline Skills Strength & $.79(.01)$ & $-.20(.01)$ & $-.14(.01)$ & $.80(.01)$ & $-.20(.01)$ & $-.14(.01)$ & $.80(.01)$ & $-.19(.01)$ & $-.14(.01)$ \\
\hline Stability Risk & $-.01(.01)$ & $.27(.01)$ & $.79(.01)$ & $-.01(.01)$ & $.27(.01)$ & $.78(.01)$ & $-.01(.01)$ & $.27(.01)$ & $.78(.01)$ \\
\hline Stability Strength & $.41(.01)$ & $.07(.01)$ & $-.72(.01)$ & $.43(.01)$ & $.04(.01)$ & $-.69(.01)$ & $.42(.01)$ & $.03(.01)$ & $-.70(.01)$ \\
\hline
\end{tabular}

Note. Substance use dynamic risk domain and the Family dynamic risk domain did not load onto any factor across all timepoints, and as such, were removed from the final factor structure. The Social Influences Risk domain loaded onto Factor 2 and Factor 3, however, theoretically it made more sense to include the Social Influences Risk domain in Factor 3. Similarly, the Stability Strength domain loaded onto Factor 1 and Factor 3, however, theoretically it made more sense to include the Stability Strength domain in Factor 1. 
Table 2E

Varimax Rotated Loadings and Standard Errors from EFAs across Three Timepoints for Women

\begin{tabular}{|c|c|c|c|c|c|c|c|c|c|}
\hline \multirow[b]{2}{*}{ SPIn Dynamic Domains } & \multicolumn{3}{|c|}{ Time $1_{\text {(initial) }}$} & \multicolumn{3}{|c|}{ Time 2 (3-8 months) } & \multicolumn{3}{|c|}{ Time 3 (9-14 months) } \\
\hline & F1 & $\mathrm{F} 2$ & F3 & F1 & $\mathrm{F} 2$ & F3 & F1 & $\mathrm{F} 2$ & F3 \\
\hline Aggression Risk & $-.09(.02)$ & $.64(.02)$ & $.03(.02)$ & $-.11(.02)$ & $.59(.02)$ & $.06(.02)$ & $-.12(.02)$ & $.53(.02)$ & $.10(.02)$ \\
\hline Substance Use Risk & -- & -- & -- & -- & -- & -- & -- & -- & -- \\
\hline Social Influences Risk & $-.03(.02)$ & $.35(.02)$ & $.48(.02)$ & $-.05(.02)$ & $.37(.02)$ & $.47(.02)$ & $-.05(.02)$ & $.42(.03)$ & $.45(.02)$ \\
\hline Family Risk & -- & -- & -- & -- & -- & -- & -- & -- & -- \\
\hline Family Strength & $.61(.02)$ & $-.02(.02)$ & $-.16(.02)$ & $.61(.02)$ & $.00(.02)$ & $-.18(.02)$ & $.61(.02)$ & $.01(.02)$ & $-.19(.02)$ \\
\hline Employment Risk & $-.07(.02)$ & $.37(.02)$ & $.49(.02)$ & $-.07(.02)$ & $.36(.02)$ & $.52(.02)$ & $-.07(.02)$ & $.42(.02)$ & $.51(.02)$ \\
\hline Employment Strength & $.58(.02)$ & $-.01(.02)$ & $-.31(.02)$ & $.56(.02)$ & $-.01(.02)$ & $-.35(.02)$ & $.56(.02)$ & $-.02(.02)$ & $-.36(.02)$ \\
\hline Attitudes Risk & $-.16(.02)$ & $.62(.02)$ & $.17(.02)$ & $-.16(.02)$ & $.59(.02)$ & $.20(.02)$ & $-.17(.02)$ & $.67(.02)$ & $.17(.02)$ \\
\hline Stability Risk & $.02(.02)$ & $.23(.02)$ & $.85(.02)$ & $.00(.02)$ & $.23(.02)$ & $.85(.01)$ & $-.00(.02)$ & $.28(.02)$ & $.83(.02)$ \\
\hline Stability Strength & $.49(.02)$ & $.05(.02)$ & $-.61(.02)$ & $.49(.02)$ & $.04(.02)$ & $-.63(.02)$ & $.49(.02)$ & $.02(.02)$ & $-.64(.02)$ \\
\hline
\end{tabular}

Note. Substance use dynamic risk domain and the Family dynamic risk domain did not load onto any factor across all timepoints, and as such, were removed from the final Factor structure. The Stability Strength domain loaded onto Factor 1 and Factor 3, however, theoretically it made more sense to include the Stability Strength domain in Factor 1. 
Table 3E

Internal Consistency of the Three-Factor Structure for Men and Women across Three Timepoints

\begin{tabular}{lccccccc}
\hline & & \multicolumn{3}{c}{ Men } & \multicolumn{3}{c}{ Women } \\
\cline { 3 - 9 } Factor & \# of Items & Time 1 & Time 2 & Time 3 & Time 1 & Time 2 & Time 3 \\
\hline Factor 1 - Protective & 7 & .84 & .84 & .84 & .85 & .86 & .86 \\
Factor 2 - Intrapersonal Risk & 3 & .77 & .77 & .77 & .73 & .73 & .70 \\
Factor 3 - Interpersonal & 3 & .62 & .63 & .64 & .70 & .71 & .72 \\
$\quad$ Stability Risk & & & & & & &
\end{tabular}

Notes. Cronbach's alpha is presented for each timepoint. 


\section{Appendix F \\ Measurement Invariance Results for 2-Factor Structure of the SPIn used in Practice Over Three Timepoints for Men and Women}

In practice, the dynamic domains of the SPIn are split into two groups: total dynamic strength and total dynamic risk. As such, the measurement invariance of these two factors, over three timepoints, were examined. Similar to the way measurement invariance was tested using the three-factor structure (that emerged in the EFAs over time), the analyses will be computed for men and women separately to see if the invariance is found for both genders. Notably, dynamic strength is the same in both the three-factor structure previously assessed and the two-factor structure.

\section{Dynamic Strength}

The total dynamic strength score is calculated using dynamic scores from the aggression/violence, social influences, family, employment, attitudes, skills, and stability strength domains. For men, as seen in Table 1F, there was an improvement in model fit when comparing the scalar to the configural model $\left(\mathrm{CFI}_{\text {change }}=.001, \mathrm{TLI}_{\text {change }}=.002\right.$, RMSEA $\left._{\text {change }}=.013\right)$. For the women $($ see Table $2 \mathrm{~F})$, there was no change in the CFI between the scalar and configural models. However, the other fit indices indicated slight improvement when comparing the scalar to the configural model $\left(\mathrm{TLI}_{\text {change }}=.001\right.$, $\left.\operatorname{RMSEA}_{\text {change }}=.003\right)$, indicating that the scalar model fit the data better. In other words, for both men and women, the SPIn total dynamic strength score is assessing the same construct over time.

\section{Dynamic Risk}

The total dynamic risk score is calculated using dynamic scores from the aggression/violence, social influences, substance use, family, employment, attitudes, 
skills, and stability risk domains. For the men, as seen in Table 1F, there was no difference in CFI between the two models, however, the other fit indices indicated a slight improvement in model fit when comparing the scalar to the configural model $\left(\mathrm{TLI}_{\text {change }}=.001, \mathrm{RMSEA}_{\text {change }}=.003\right)$. For the women, as seen in Table $2 \mathrm{~F}$, the CFI and TLI indices indicated that there was no change in model fit between the configural and scalar models, however, the RMSEA indicated slight improvement when comparing the scalar to the configural model $\left(\mathrm{RMSEA}_{\mathrm{chang}}=.002\right)$. Taken together, the results indicate that the scalar model fit the data better. In other words, for both men and women, the SPIn total dynamic risk score is assessing the same construct over time.

Table 1F

Model Fit Indices and Nested Model Comparisons for Testing Invariance of Dynamic SPIn Constructs Used in Practice for Men

\begin{tabular}{lccc}
\hline Dynamic Strength $^{1}$ & CFI & TLI & RMSEA [95\% CI] \\
\hline Configural $^{2}$ & .995 & .994 & $.080[.078, .081]$ \\
Scalar $^{3}$ & .996 & .996 & $.067[.065, .070]$ \\
\hline Dynamic Risk & CFI & TLI & RMSEA [95\% CI] \\
\hline Configural & .997 & .996 & $.060[.058, .061]$ \\
Scalar & .997 & .997 & $.057[.056, .058]$ \\
\hline
\end{tabular}

Note. CFI = Comparative Fit Index. TLI $=$ Tucker-Lewis Index. RMSEA $=$ Rooted Mean Square Error of Approximation. CI = Confidence Interval. ${ }^{1}$ Dynamic Strength $=$ same factor structure as the 'strength' factor from the three-factor solution that emerged from the EFAs. ${ }^{2}$ Configural $=$ No equality constraints. ${ }^{3}$ Scalar $=$ Factor loadings and intercepts constrained to be equal across all timepoints. 
Table $2 \mathrm{~F}$

Model Fit Indices and Nested Model Comparisons for Testing Invariance of Dynamic SPIn Constructs Used in Practice for Women

\begin{tabular}{lccc}
\hline Dynamic Strength $^{1}$ & CFI & TLI & RMSEA [95\% CI] \\
\hline Configural $^{2}$ & .997 & .996 & $.056[.078, .081]$ \\
Scalar $^{3}$ & .997 & .997 & $.053[.050, .055]$ \\
\hline Dynamic Risk & CFI & TLI & RMSEA [95\% CI] \\
\hline Configural & .997 & .997 & $.050[.047, .052]$ \\
Scalar & .997 & .997 & $.048[.045, .050]$
\end{tabular}

Note. CFI $=$ Comparative Fit Index. TLI $=$ Tucker-Lewis Index. RMSEA $=$ Rooted Mean Square Error of Approximation. $\mathrm{CI}=$ Confidence Interval. Strength $=$ same factor structure as the 'strength' factor from the three-factor solution that emerged from the EFAs. ${ }^{2}$ Configural $=$ No equality constraints. ${ }^{3}$ Scalar $=$ Factor loadings and intercepts constrained to be equal across all timepoints. 


\section{Appendix G}

\section{Analyses for Study 2}

Table $1 \mathrm{G}$

Breakdown of Analyses for Study 2

\begin{tabular}{|c|c|c|}
\hline Analyses & Statistical Method & Software \\
\hline Descriptives & Frequencies & SPSS \\
\hline \multirow[t]{3}{*}{ Basic Data Cleaning } & T-test/ANOVA & \\
\hline & Chi-square & \\
\hline & Correlations & \\
\hline Latent Profile Analysis & Step 1: Run LPA at Time 1 & MPlus \\
\hline \multirow[t]{5}{*}{ Latent Transition Analysis } & $\begin{array}{l}\text { Step 2: Run LPA at Time } 2 \text { and } \\
\text { Time } 3 \text { (Part of LTA) }\end{array}$ & \\
\hline & Step 3: Examination of covariates & \\
\hline & $\begin{array}{l}\text { Step 4: Examination of auxiliary } \\
\text { variables }\end{array}$ & \\
\hline & $\begin{array}{l}\text { Step 5: Examine transitional } \\
\text { probabilities between profiles }{ }^{1} \text { (main } \\
\text { component of LTA) }\end{array}$ & \\
\hline & $\begin{array}{l}\text { Step 6: Relationship between profiles } \\
\text { and distal outcomes (using logistic } \\
\text { regression) }\end{array}$ & \\
\hline
\end{tabular}

Note. Transitional probabilities between timepoints can only be assessed if the same typological structure emerges at each timepoint. 


\section{Appendix H}

\section{Means, Standard Deviations and Ranges for Variables Included in LPAs at Each \\ Timepoint for Each Gender}

Table $1 \mathrm{H}$

Descriptive Statistics for Variables in LPA at Time 1, by Gender

\begin{tabular}{|c|c|c|c|c|c|c|}
\hline \multirow{2}{*}{$\begin{array}{l}\text { Continuous and Count } \\
\text { Variables }\end{array}$} & \multicolumn{3}{|c|}{ Men } & \multicolumn{3}{|c|}{ Women } \\
\hline & $M$ & $S D$ & Range & $M$ & $S D$ & Range \\
\hline Criminal history & 6.09 & 5.32 & $0-21$ & 4.13 & 4.33 & $0-21$ \\
\hline Aggression - Risk & 0.35 & 1.04 & $0-8$ & 0.26 & 0.87 & $0-8$ \\
\hline Aggression - Strength & 1.87 & 2.02 & $0-8$ & 1.96 & 2.17 & $0-8$ \\
\hline Substance use - Risk & 6.11 & 7.45 & $0-128$ & 5.75 & 7.56 & $0-84$ \\
\hline Social influence - Risk & 2.61 & 3.12 & $0-25$ & 2.48 & 3.17 & $0-21$ \\
\hline Social influence - Strength & 4.66 & 3.35 & $0-15$ & 4.58 & 3.32 & $0-15$ \\
\hline Family - Risk & 4.44 & 4.16 & $0-23$ & 4.29 & 4.13 & $0-25$ \\
\hline Family - Strength & 3.64 & 2.79 & $0-14$ & 3.71 & 2.82 & $0-14$ \\
\hline Employment - Risk & 0.57 & 1.57 & $0-13$ & 1.08 & 2.13 & $0-13$ \\
\hline Employment - Strength & 3.59 & 3.01 & $0-12$ & 2.38 & 2.58 & $0-12$ \\
\hline Attitudes - Risk & 1.25 & 2.15 & $0-17$ & 0.83 & 1.66 & $0-12$ \\
\hline Attitudes - Strength & 5.48 & 4.35 & $0-18$ & 6.20 & 4.65 & $0-18$ \\
\hline Cognitive/social Risk & 0.87 & 1.93 & $0-16$ & 0.66 & 1.60 & $0-15$ \\
\hline Cognitive/social Strength & 3.77 & 3.81 & $0-16$ & 4.03 & 4.00 & $0-16$ \\
\hline Stability - Risk & 1.27 & 1.82 & $0-13$ & 1.72 & 2.05 & $0-13$ \\
\hline Stability - Strength & 4.06 & 1.64 & $0-7$ & 3.58 & 1.58 & $0-7$ \\
\hline Mental health - Flag & 0.73 & 0.90 & $0-2$ & 1.12 & 0.91 & $0-2$ \\
\hline $\mathrm{ACE}^{1}$ & 0.92 & 1.32 & $0-7$ & 1.46 & 1.74 & $0-8$ \\
\hline \multicolumn{7}{|l|}{ Covariates } \\
\hline Total Static Risk Score & 22.31 & 16.96 & $0-109$ & 18.53 & 14.88 & $0-94$ \\
\hline Age & 34.39 & 11.60 & $16-82$ & 33.34 & 10.99 & $17-74$ \\
\hline
\end{tabular}

Note. $M=$ mean. $S D=$ standard deviation. ${ }^{1} \mathrm{ACE}=$ Adverse Childhood Experiences. 
Table $2 \mathrm{H}$

Descriptive Statistics for Variables in LPA at Time 2, by Gender

\begin{tabular}{|c|c|c|c|c|c|c|}
\hline \multirow{2}{*}{$\begin{array}{l}\text { Continuous and Count } \\
\text { Variables }\end{array}$} & \multicolumn{3}{|c|}{ Men } & \multicolumn{3}{|c|}{ Women } \\
\hline & $M$ & $S D$ & Range & $M$ & $S D$ & Range \\
\hline Criminal history & 6.84 & 5.54 & $0-21$ & 4.84 & 4.74 & $0-21$ \\
\hline Aggression - Risk & 0.36 & 1.06 & $0-8$ & 0.25 & 0.85 & $0-8$ \\
\hline Aggression - Strength & 1.96 & 2.04 & $0-8$ & 2.10 & 2.18 & $0-8$ \\
\hline Substance use - Risk & 6.00 & 7.02 & $0-128$ & 5.57 & 7.00 & $0-99$ \\
\hline Social influence - Risk & 2.94 & 3.49 & $0-26$ & 2.79 & 3.45 & $0-21$ \\
\hline Social influence - Strength & 4.66 & 3.36 & $0-15$ & 4.59 & 3.30 & $0-15$ \\
\hline Family - Risk & 4.60 & 4.21 & $0-26$ & 4.52 & 4.26 & $0-23$ \\
\hline Family - Strength & 3.68 & 2.79 & $0-14$ & 3.76 & 2.80 & $0-14$ \\
\hline Employment - Risk & 0.69 & 1.70 & $0-13$ & 1.26 & 2.33 & $0-13$ \\
\hline Employment - Strength & 3.61 & 3.03 & $0-12$ & 2.38 & 2.59 & $0-12$ \\
\hline Attitudes - Risk & 1.34 & 2.30 & $0-18$ & 0.88 & 1.72 & $0-16$ \\
\hline Attitudes - Strength & 5.61 & 4.44 & $0-18$ & 6.35 & 4.70 & $0-18$ \\
\hline Cognitive/social Risk & 0.96 & 2.07 & $0-16$ & 0.66 & 1.65 & $0-16$ \\
\hline Cognitive/social Strength & 3.86 & 3.86 & $0-16$ & 4.17 & 4.06 & $0-16$ \\
\hline Stability - Risk & 1.46 & 2.01 & $0-13$ & 1.95 & 2.19 & $0-13$ \\
\hline Stability - Strength & 4.03 & 1.68 & $0-7$ & 3.55 & 1.60 & $0-7$ \\
\hline Mental health - Flag & 0.74 & 0.89 & $0-2$ & 1.12 & 0.91 & $0-2$ \\
\hline $\mathrm{ACE}^{1}$ & 1.02 & 1.38 & $0-8$ & 1.58 & 1.77 & $0-8$ \\
\hline \multicolumn{7}{|l|}{ Covariates } \\
\hline Total Static Risk Score & 22.05 & 16.78 & $0-109$ & 18.34 & 14.77 & $0-94$ \\
\hline Age & 34.39 & 11.60 & $16-82$ & 33.35 & 11.00 & $17-75$ \\
\hline
\end{tabular}

Note. $M=$ mean. $S D=$ standard deviation. ${ }^{1} \mathrm{ACE}=$ Adverse Childhood Experiences. 
Table $3 \mathrm{H}$

Descriptive Statistics for Variables in LPA at Time 3, by Gender

\begin{tabular}{|c|c|c|c|c|c|c|}
\hline \multirow{2}{*}{$\begin{array}{l}\text { Continuous and Count } \\
\text { Variables }\end{array}$} & \multicolumn{3}{|c|}{ Men } & \multicolumn{3}{|c|}{ Women } \\
\hline & $M$ & $S D$ & Range & $M$ & $S D$ & Range \\
\hline Criminal history & 6.95 & 5.56 & $0-21$ & 4.97 & 4.78 & $0-21$ \\
\hline Aggression - Risk & 0.34 & 1.03 & $0-8$ & 0.25 & 0.86 & $0-8$ \\
\hline Aggression - Strength & 2.02 & 2.05 & $0-8$ & 2.16 & 2.20 & $0-8$ \\
\hline Substance use - Risk & 5.96 & 6.61 & $0-128$ & 5.36 & 6.71 & $0-99$ \\
\hline Social influence - Risk & 2.92 & 3.50 & $0-25$ & 2.78 & 3.47 & $0-22$ \\
\hline Social influence - Strength & 4.73 & 3.38 & $0-15$ & 4.66 & 3.33 & $0-15$ \\
\hline Family - Risk & 4.62 & 4.20 & $0-26$ & 4.54 & 4.22 & $0-24$ \\
\hline Family - Strength & 3.74 & 2.82 & $0-14$ & 3.81 & 2.80 & $0-14$ \\
\hline Employment - Risk & 0.71 & 1.74 & $0-13$ & 1.30 & 2.37 & $0-13$ \\
\hline Employment - Strength & 3.70 & 3.07 & $0-12$ & 2.42 & 2.65 & $0-12$ \\
\hline Attitudes - Risk & 1.33 & 2.30 & $0-18$ & 0.89 & 1.77 & $0-16$ \\
\hline Attitudes - Strength & 5.70 & 4.50 & $0-18$ & 6.48 & 4.75 & $0-18$ \\
\hline Cognitive/social Risk & 0.94 & 2.05 & $0-16$ & 0.68 & 1.67 & $0-16$ \\
\hline Cognitive/social Strength & 3.98 & 3.94 & $0-16$ & 4.27 & 4.13 & $0-16$ \\
\hline Stability - Risk & 1.45 & 2.01 & $0-12$ & 1.96 & 2.23 & $0-13$ \\
\hline Stability - Strength & 4.07 & 1.69 & $0-7$ & 3.60 & 1.61 & $0-7$ \\
\hline Mental health - Flag & 0.73 & 0.89 & $0-2$ & 1.13 & 0.90 & $0-2$ \\
\hline $\mathrm{ACE}^{1}$ & 1.02 & 1.38 & $0-8$ & 1.58 & 1.77 & $0-8$ \\
\hline \multicolumn{7}{|l|}{ Covariates } \\
\hline Total Static Risk Score & 25.43 & 18.67 & $0-125$ & 21.18 & 16.29 & $0-102$ \\
\hline Age & 34.76 & 11.43 & $16-83$ & 33.38 & 10.81 & $17-75$ \\
\hline
\end{tabular}

Note. $M=$ mean. $S D=$ standard deviation. ${ }^{1} \mathrm{ACE}=$ Adverse Childhood Experiences. 


\section{Appendix I}

\section{Supplemental Tables for Covariates (Age and Total Static Risk Scores) For Women and Men at Each Timepoint}

Table 1I

Categorical Latent Variable Regression of Profile on Age at Time 1 for Women

\begin{tabular}{cccc}
\hline Reference: Profile 1 & Odds Ratio & $(\boldsymbol{S E})$ & $\boldsymbol{p}$ \\
\hline Profile 2 on age & 1.014 & $(.006)$ & .017 \\
Profile 3 on age & 1.015 & $(.008)$ & .063 \\
\hline Reference: Profile 3 & Odds Ratio & $(\boldsymbol{S E})$ & $\boldsymbol{p}$ \\
\hline Profile 1 on age & 0.985 & $(.008)$ & .059 \\
Profile 2 on age & 0.999 & $(.007)$ & .880 \\
\hline
\end{tabular}

Note. $S E=$ Standard error. Profile $1=$ Low risk/low strength, Profile 2 = Genderresponsive, low risk/high strength, and Profile $3=$ Aggressive, complex need/low strength.

Table 2I

Categorical Latent Variable Regression of Profile on Age at Time 2 for Women

\begin{tabular}{cccc}
\hline Reference: Profile 1 & Odds Ratio & $(\boldsymbol{S E})$ & $\boldsymbol{p}$ \\
\hline Profile 2 on age & 1.011 & $(.006)$ & .067 \\
Profile 3 on age & 1.008 & $(.008)$ & .331 \\
\hline Reference: Profile 3 & Odds Ratio & $(\boldsymbol{S E})$ & $\boldsymbol{p}$ \\
\hline Profile 1 on age & 0.992 & $(.008)$ & .328 \\
Profile 2 on age & 1.003 & $(.007)$ & .704
\end{tabular}

Note. $S E=$ Standard error. Profile $1=$ Low risk/low strength, Profile $2=$ Genderresponsive, low risk/high strength, and Profile $3=$ Aggressive, complex need/low strength. 
Table 3I

Categorical Latent Variable Regression of Profile on Age at Time 3 for Women

\begin{tabular}{cccc}
\hline Reference: Profile 1 & Odds Ratio & $(S E)$ & $p$ \\
\hline Profile 2 on age & 1.009 & $(.006)$ & .102 \\
Profile 3 on age & 1.007 & $(.008)$ & .415 \\
\hline Reference: Profile 3 & Odds Ratio & $(S E)$ & $p$ \\
\hline Profile 1 on age & 0.993 & $(.008)$ & .412 \\
Profile 2 on age & 1.003 & $(.008)$ & .727
\end{tabular}

Note. $S E=$ Standard error. Profile $1=$ Low risk/low strength, Profile $2=$ Genderresponsive, low risk/high strength, and Profile $3=$ Aggressive, complex need/low strength. 
Table 4I

Categorical Latent Variable Regression of Profile on Age at Time 1 for Men

\begin{tabular}{|c|c|c|c|}
\hline Reference: Profile 1 & Odds Ratio & $(S E)$ & $p$ \\
\hline Profile 2 on age & 1.001 & $(.005)$ & .819 \\
\hline Profile 3 on age & 1.001 & $(.004)$ & .938 \\
\hline Profile 4 on age & 1.003 & $(.004)$ & .468 \\
\hline Profile 5 on age & 0.995 & $(.007)$ & .468 \\
\hline Reference: Profile 3 & Odds Ratio & $(S E)$ & $p$ \\
\hline Profile 1 on age & 1.000 & $(.004)$ & .938 \\
\hline Profile 2 on age & 1.001 & $(.006)$ & .874 \\
\hline Profile 4 on age & 1.002 & $(.003)$ & .453 \\
\hline Profile 5 on age & 0.994 & $(.007)$ & .460 \\
\hline Reference: Profile 4 & Odds Ratio & $(S E)$ & $p$ \\
\hline Profile 1 on age & 0.997 & $(.004)$ & .467 \\
\hline Profile 2 on age & 0.999 & $(.006)$ & .810 \\
\hline Profile 3 on age & 0.998 & $(.003)$ & .452 \\
\hline Profile 5 on age & 0.992 & $(.007)$ & .284 \\
\hline Reference: Profile 5 & Odds Ratio & $(S E)$ & $p$ \\
\hline Profile 1 on age & 1.005 & $(.007)$ & .470 \\
\hline Profile 2 on age & 1.007 & $(.009)$ & .448 \\
\hline Profile 3 on age & 1.006 & $(.008)$ & .462 \\
\hline Profile 4 on age & 1.008 & $(.007)$ & .288 \\
\hline
\end{tabular}

Note. $S E=$ Standard error. Profile $1=$ Aggressive, low risk/low strength, Profile $2=$ Aggressive, complex need/low strength, Profile 3 = Moderate risk/moderate strength, Profile $4=$ Low risk/high strength, and Profile $5=$ Low stability, complex need/low strength. 
Table 5I

Categorical Latent Variable Regression of Profile on Age at Time 2 for Men

\begin{tabular}{cccc}
\hline Reference: Profile 1 & Odds Ratio & $($ SE) & $\boldsymbol{p}$ \\
\hline Profile 2 on age & 1.001 & $(.006)$ & .922 \\
Profile 3 on age & 0.996 & $(.004)$ & .308 \\
Profile 4 on age & 0.998 & $(.003)$ & .949 \\
Profile 5 on age & 0.996 & $(.008)$ & .584 \\
\hline Reference: Profile 3 & Odds Ratio & $(\boldsymbol{S E})$ & $\boldsymbol{p}$ \\
\hline Profile 1 on age & 1.004 & $(.004)$ & .310 \\
Profile 2 on age & 1.005 & $(.006)$ & .465 \\
Profile 4 on age & 1.002 & $(.003)$ & .604 \\
Profile 5 on age & 1.000 & $(.008)$ & .965 \\
\hline Profile 1 on age & 1.002 & $(.003)$ & $\boldsymbol{p}$ \\
\hline Profile 2 on age & 1.003 & $(.006)$ & .495 \\
Profile 3 on age & 0.998 & $(.003)$ & .604 \\
Profile 5 on age & 0.998 & $(.008)$ & .797 \\
\hline Prefence: Profile 4 & Odds Ratio & $(\boldsymbol{S E})$ & $\boldsymbol{p}$ \\
\hline Profile 1 on age & 1.004 & $(.008)$ & .586 \\
Profile 2 on age & 1.005 & $(.009)$ & .592 \\
Profile 3 on age & 1.000 & $(.008)$ & .965 \\
\hline
\end{tabular}

Note. $S E=$ Standard error. Profile $1=$ Low risk/low strength, Profile $2=$ Aggressive, complex need/low strength, Profile $3=$ Moderate risk/moderate strength, Profile $4=$ Low risk/high strength, and Profile $5=$ Low stability, complex need/low strength. 
Table 6I

Categorical Latent Variable Regression of Profile on Age at Time 3 for Men

\begin{tabular}{|c|c|c|c|}
\hline Reference: Profile 1 & Odds Ratio & $(S E)$ & $p$ \\
\hline Profile 2 on age & 1.009 & $(.007)$ & .171 \\
\hline Profile 3 on age & 1.000 & $(.003)$ & .914 \\
\hline Profile 4 on age & 1.005 & $(.004)$ & .209 \\
\hline Profile 5 on age & 1.000 & $(.008)$ & .959 \\
\hline Profile 6 on age & 1.006 & $(.005)$ & .186 \\
\hline Reference: Profile 2 & Odds Ratio & $(S E)$ & $p$ \\
\hline Profile 1 on age & 0.991 & $(.007)$ & .167 \\
\hline Profile 3 on age & 0.991 & $(.006)$ & .165 \\
\hline Profile 4 on age & 0.995 & $(.007)$ & .507 \\
\hline Profile 5 on age & 0.991 & $(.010)$ & .359 \\
\hline Profile 6 on age & 0.997 & $(.007)$ & .627 \\
\hline Reference: Profile 4 & Odds Ratio & $(S E)$ & $p$ \\
\hline $\begin{array}{l}\text { Profile } 1 \text { on age } \\
\end{array}$ & 0.995 & $(.004)$ & .207 \\
\hline Profile 2 on age & 1.005 & $(.007)$ & .509 \\
\hline Profile 3 on age & 0.996 & $(.004)$ & .255 \\
\hline Profile 5 on age & 0.996 & $(.008)$ & .604 \\
\hline Profile 6 on age & 1.001 & $(.005)$ & .777 \\
\hline Reference: Profile 5 & Odds Ratio & $(S E)$ & $p$ \\
\hline Profile 1 on age & 1.000 & $(.008)$ & .959 \\
\hline Profile 2 on age & 1.009 & $(.010)$ & .364 \\
\hline Profile 3 on age & 1.000 & $(.008)$ & .993 \\
\hline Profile 4 on age & 1.004 & $(.008)$ & .606 \\
\hline Profile 6 on age & 1.006 & $(.009)$ & .506 \\
\hline Reference: Profile 6 & Odds Ratio & $(S E)$ & $p$ \\
\hline Profile 1 on age & 0.994 & $(.005)$ & .184 \\
\hline Profile 2 on age & 1.003 & $(.007)$ & .628 \\
\hline Profile 3 on age & 0.994 & $(.004)$ & .184 \\
\hline Profile 4 on age & 0.999 & $(.005)$ & .777 \\
\hline Profile 5 on age & 0.994 & $(.008)$ & .504 \\
\hline
\end{tabular}

Note. $S E=$ Standard error. Profile $1=$ Low risk/low strength, Profile $2=$ Aggressive, complex need/low strength, Profile $3=$ Moderate risk/moderate strength, Profile $4=$ Low risk/high strength, Profile $5=$ Low stability, complex need/low strength, and Profile $6=$ Moderate complex need/low strength. 
Table 7I

Categorical Latent Variable Regression of Profile on Total Static Risk Scores at Time 1 for Women

\begin{tabular}{cccc}
\hline Reference: Profile 1 & Odds Ratio & $(S E)$ & $p$ \\
\hline Profile 2 on static & 1.111 & $(.013)$ & $<.001$ \\
Profile 3 on static & 1.204 & $(.017)$ & $<.001$ \\
\hline Reference: Profile 3 & Odds Ratio & $(\boldsymbol{S E})$ & $\boldsymbol{p}$ \\
\hline Profile 1 on static & 0.830 & $(.012)$ & $<.001$ \\
Profile 2 on static & 0.922 & $(.009)$ & $<.001$
\end{tabular}

Note. $S E=$ Standard error. Profile $1=$ Low risk/low strength, Profile 2 = Genderresponsive, low risk/high strength, and Profile $3=$ Aggressive, complex need/low strength.

Table 8I

Categorical Latent Variable Regression of Profile on Total Static Risk Scores at Time 2 for Women

\begin{tabular}{cccc}
\hline Reference: Profile 1 & Odds Ratio & $(S E)$ & $p$ \\
\hline Profile 2 on static & 1.099 & $(.014)$ & $<.001$ \\
Profile 3 on static & 1.189 & $(.019)$ & $<.001$ \\
\hline Reference: Profile 3 & Odds Ratio & $(\boldsymbol{S E})$ & $\boldsymbol{p}$ \\
\hline Profile 1 on static & 0.841 & $(.014)$ & $<.001$ \\
Profile 2 on static & 0.925 & $(.008)$ & $<.001$ \\
\hline
\end{tabular}

Note. $S E=$ Standard error. Profile $1=$ Low risk/low strength, Profile 2 = Genderresponsive, low risk/high strength, and Profile $3=$ Aggressive, complex need/low strength. 
Table 9I

Categorical Latent Variable Regression of Profile on Total Static Risk Scores at Time 3 for Women

\begin{tabular}{cccc}
\hline Reference: Profile 1 & Odds Ratio & $(\boldsymbol{S E})$ & $\boldsymbol{p}$ \\
\hline Profile 2 on static & 1.095 & $(.014)$ & $<.001$ \\
Profile 3 on static & 1.190 & $(.018)$ & $<.001$ \\
\hline Reference: Profile 3 & Odds Ratio & $(\boldsymbol{S E})$ & $\boldsymbol{p}$ \\
\hline Profile 1 on static & 0.841 & $(.013)$ & $<.001$ \\
Profile 2 on static & 0.920 & $(.008)$ & $<.001$
\end{tabular}

Note. $S E=$ Standard error. Profile $1=$ Low risk/low strength, Profile 2 = Genderresponsive, low risk/high strength, and Profile $3=$ Aggressive, complex need/low strength. 
Table 10I

Categorical Latent Variable Regression of Profile on Total Static Risk Score at Time 1 for Men

\begin{tabular}{|c|c|c|c|}
\hline Reference: Profile 1 & Odds Ratio & $(S E)$ & $p$ \\
\hline Profile 2 on static & 1.023 & $(.005)$ & $<.001$ \\
\hline Profile 3 on static & 0.823 & $(.004)$ & $<.001$ \\
\hline Profile 4 on static & 0.926 & $(.006)$ & $<.001$ \\
\hline Profile 5 on static & 1.018 & $(.005)$ & $<.001$ \\
\hline Reference: Profile 3 & Odds Ratio & $(S E)$ & $p$ \\
\hline Profile 1 on static & 1.216 & $(.0047)$ & $<.001$ \\
\hline Profile 2 on static & 1.244 & $(.008)$ & $<.001$ \\
\hline Profile 4 on static & 1.125 & $(.006)$ & $<.001$ \\
\hline Profile 5 on static & 1.237 & $(.008)$ & $<.001$ \\
\hline Reference: Profile 4 & Odds Ratio & $(S E)$ & $p$ \\
\hline Profile 1 on static & 1.080 & $(.007)$ & $<.001$ \\
\hline Profile 2 on static & 1.105 & $(.007)$ & $<.001$ \\
\hline Profile 3 on static & 0.889 & $(.005)$ & $<.001$ \\
\hline Profile 5 on static & 1.099 & $(.007)$ & $<.001$ \\
\hline Reference: Profile 5 & Odds Ratio & $(S E)$ & $p$ \\
\hline Profile 1 on static & 0.983 & $(.004)$ & $<.001$ \\
\hline Profile 2 on static & 1.005 & $(.006)$ & .385 \\
\hline Profile 3 on static & 0.808 & $(.005)$ & $<.001$ \\
\hline Profile 4 on static & 0.910 & $(.006)$ & $<.001$ \\
\hline
\end{tabular}

Note. $S E=$ Standard error. Profile $1=$ Low risk/low strength, Profile $2=$ Aggressive, complex need/low strength, Profile $3=$ Moderate risk/moderate strength, Profile $4=$ Low risk/high strength, and Profile 5 = Low stability, complex need/low strength. 
Table 11I

Categorical Latent Variable Regression of Profile on Total Static Risk Score at Time 2 for Men

\begin{tabular}{|c|c|c|c|}
\hline Reference: Profile 1 & Odds Ratio & $(S E)$ & $p$ \\
\hline Profile 2 on static & 1.024 & $(.006)$ & $<.001$ \\
\hline Profile 3 on static & 0.826 & $(.007)$ & $<.001$ \\
\hline Profile 4 on static & 0.937 & $(.006)$ & $<.001$ \\
\hline Profile 5 on static & 1.012 & $(.006)$ & $<.001$ \\
\hline Reference: Profile 3 & Odds Ratio & $(S E)$ & $p$ \\
\hline Profile 1 on static & 1.211 & $(.006)$ & $<.001$ \\
\hline Profile 2 on static & 1.225 & $(.006)$ & $<.001$ \\
\hline Profile 4 on static & 1.134 & $(.007)$ & $<.001$ \\
\hline Profile 5 on static & 1.239 & $(.006)$ & $<.001$ \\
\hline Reference: Profile 4 & Odds Ratio & $(S E)$ & $p$ \\
\hline Profile 1 on static & 1.080 & $(.006)$ & $<.001$ \\
\hline Profile 2 on static & 1.068 & $(.005)$ & $<.001$ \\
\hline Profile 3 on static & 0.882 & $(.007)$ & $<.001$ \\
\hline Profile 5 on static & 1.093 & $(.005)$ & $<.001$ \\
\hline Reference: Profile 5 & Odds Ratio & $(S E)$ & $p$ \\
\hline Profile 1 on static & 1.012 & $(.006)$ & .035 \\
\hline Profile 2 on static & 0.988 & $(.008)$ & .098 \\
\hline Profile 3 on static & 0.926 & $(.009)$ & $<.001$ \\
\hline Profile 4 on static & 1.012 & $(.007)$ & $<.001$ \\
\hline
\end{tabular}

Note. $S E=$ Standard error. Profile $1=$ Low risk/low strength, Profile $2=$ Aggressive, complex need/low strength, Profile $3=$ Moderate risk/moderate strength, Profile $4=$ Low risk/high strength, and Profile 5 = Low stability, complex need/low strength. 
Table 12I

Categorical Latent Variable Regression of Profile on Total Static Risk Score at Time 3 for Men

\begin{tabular}{|c|c|c|c|}
\hline Reference: Profile 1 & Odds Ratio & $(S E)$ & $p$ \\
\hline Profile 2 on static & 1.226 & $(.009)$ & $<.001$ \\
\hline Profile 3 on static & 1.144 & $(.006)$ & $<.001$ \\
\hline Profile 4 on static & 1.112 & $(.008)$ & $<.001$ \\
\hline Profile 5 on static & 1.210 & $(.008)$ & $<.001$ \\
\hline Profile 6 on static & 1.207 & $(.006)$ & $<.001$ \\
\hline Reference: Profile 2 & Odds Ratio & $(S E)$ & $p$ \\
\hline Profile 1 on static & 0.815 & $(.006)$ & $<.001$ \\
\hline Profile 3 on static & 0.933 & $(.005)$ & $<.001$ \\
\hline Profile 4 on static & 0.907 & $(.007)$ & $<.001$ \\
\hline Profile 5 on static & 0.986 & $(.006)$ & .015 \\
\hline Profile 6 on static & 0.984 & $(.005)$ & .004 \\
\hline Reference: Profile 4 & Odds Ratio & $(S E)$ & $p$ \\
\hline Profile 1 on static & 0.899 & $(.006)$ & $<.001$ \\
\hline Profile 2 on static & 1.103 & $(.009)$ & $<.001$ \\
\hline Profile 3 on static & 1.029 & $(.008)$ & $<.001$ \\
\hline Profile 5 on static & 1.088 & $(.008)$ & $<.001$ \\
\hline Profile 6 on static & 1.086 & $(.008)$ & $<.001$ \\
\hline Reference: Profile 5 & Odds Ratio & $(S E)$ & $p$ \\
\hline Profile 1 on static & 0.827 & $(.005)$ & $<.001$ \\
\hline Profile 2 on static & 1.014 & $(.006)$ & .016 \\
\hline Profile 3 on static & 0.946 & $(.005)$ & $<.001$ \\
\hline Profile 4 on static & 0.919 & $(.007)$ & $<.001$ \\
\hline Profile 6 on static & 0.998 & $(.005)$ & .706 \\
\hline Reference: Profile 6 & Odds Ratio & $(S E)$ & $p$ \\
\hline Profile 1 on static & 0.828 & $(.004)$ & $<.001$ \\
\hline Profile 2 on static & 1.016 & $(.006)$ & .004 \\
\hline Profile 3 on static & 0.948 & $(.004)$ & $<.001$ \\
\hline Profile 4 on static & 0.921 & $(.007)$ & $<.001$ \\
\hline Profile 5 on static & 1.002 & $(.005)$ & .706 \\
\hline
\end{tabular}

Note. $S E=$ Standard error. Profile $1=$ Low risk/low strength, Profile $2=$ Aggressive, complex need/low strength, Profile $3=$ Moderate risk/moderate strength, Profile $4=$ Low risk/high strength, Profile $5=$ Low stability, complex need/low strength, and Profile $6=$ Moderate complex need/low strength. 


\section{Appendix J}

Supplemental Tables for the Auxiliary Variable (Indigenous Status) for Women and Men at Each Timepoint

Table 1J

Chi-square Pairwise Tests on Indigenous Distribution between Profiles for Women at Each Timepoint

\section{Time 1}

\begin{tabular}{lrr}
\hline Profile Comparisons & \multicolumn{1}{c}{$\boldsymbol{\chi}^{\mathbf{2}}$} & $\boldsymbol{p}$ \\
\hline Profile 1 vs. 2 & 5.31 & .021 \\
Profile 1 vs. 3 & 25.11 & $<.001$ \\
Profile 2 vs. 3 & 9.52 & .002 \\
\hline
\end{tabular}

Time 2

\begin{tabular}{lcc}
\hline Profile Comparisons & $\boldsymbol{\chi}^{\mathbf{2}}$ & $\boldsymbol{p}$ \\
\hline Profile 1 vs. 2 & 4.94 & .026 \\
Profile 1 vs. 3 & 39.44 & $<.001$ \\
Profile 2 vs. 3 & 18.47 & $<.001$ \\
\hline
\end{tabular}

Time 3

\begin{tabular}{lcc}
\hline Profile Comparisons & $\boldsymbol{\chi}^{\mathbf{2}}$ & $\boldsymbol{p}$ \\
\hline Profile 1 vs. 2 & 5.28 & .022 \\
Profile 1 vs. 3 & 43.40 & $<.001$ \\
Profile 2 vs. 3 & 22.07 & $<.001$
\end{tabular}

Note. Profile 1 = Low risk/low strength, Profile $2=$ Gender-responsive, low risk/high strength, and Profile $3=$ Aggressive, complex need/low strength. $\chi^{2}=$ Chi-square pairwise test. 
Table 2J

Chi-square Pairwise Tests on Indigenous Distribution between Profiles for Men at Time 1

\begin{tabular}{lrc}
\hline Profile Comparisons & \multicolumn{1}{c}{$\boldsymbol{\chi}^{\mathbf{2}}$} & $\boldsymbol{p}$ \\
\hline Profile 1 vs. 2 & 10.04 & .002 \\
Profile 1 vs. 3 & 110.06 & $<.001$ \\
Profile 1 vs. 4 & 34.47 & $<.001$ \\
Profile 1 vs. 5 & 44.99 & $<.001$ \\
Profile 2 vs. 3 & 9.23 & .002 \\
Profile 2 vs. 4 & 0.06 & $\mathbf{. 0 0 6}$ \\
Profile 2 vs. 5 & 13.45 & $<.001$ \\
Profile 3 vs. 4 & 17.73 & $<.001$ \\
Profile 3 vs. 5 & 3.65 & $\mathbf{. 0 5 6}$ \\
Profile 4 vs. 5 & 16.54 & $<.001$ \\
\hline
\end{tabular}

Note. Profile 1 = Low risk/low strength, Profile $2=$ Aggressive, complex need/low strength, Profile $3=$ Moderate risk/moderate strength, Profile $4=$ Low risk/high strength, and Profile $5=$ Low stability, complex need/low strength. $\chi^{2}=$ Chi-square pairwise test. 
Table 3J

Chi-square Tests on Indigenous Distribution between Profiles for Men at Time 2 and Time 3

\begin{tabular}{lcc}
\hline & Time 2 & \\
\hline Profile Comparisons & \multicolumn{1}{c}{$\boldsymbol{\chi}^{\mathbf{2}}$} & \multicolumn{1}{c}{} \\
\hline Profile 1 vs. 2 & 13.29 & $<.001$ \\
Profile 1 vs. 3 & 95.50 & $<.001$ \\
Profile 1 vs. 4 & 41.91 & $<.001$ \\
Profile 1 vs. 5 & 44.21 & $\mathbf{. 1 5 0}$ \\
Profile 2 vs. 3 & 2.07 &. $\mathbf{7 3 6}$ \\
Profile 2 vs. 4 & 0.11 & .007 \\
Profile 2 vs. 5 & 7.22 & .002 \\
Profile 3 vs. 4 & 9.69 & .042 \\
Profile 3 vs. 5 & 4.13 & $<.001$ \\
Profile 4 vs. 5 & 13.32 & \\
\hline
\end{tabular}

\begin{tabular}{lrc}
\hline \multicolumn{3}{c}{ Time 3 } \\
\hline Profile Comparisons & $\boldsymbol{\chi}^{\mathbf{2}}$ & $\boldsymbol{p}$ \\
\hline Profile 1 vs. 2 & 9.16 & .002 \\
Profile 1 vs. 3 & 61.74 & $<.001$ \\
Profile 1 vs. 4 & 16.84 & $<.001$ \\
Profile 1 vs. 5 & 36.12 & $<.001$ \\
Profile 1 vs. 6 & 71.36 & $<.001$ \\
Profile 2 vs. 3 & 0.45 & $\mathbf{. 5 0 5}$ \\
Profile 2 vs. 4 & 0.27 & $\mathbf{. 6 0 5}$ \\
Profile 2 vs. 5 & 8.33 & .004 \\
Profile 2 vs. 6 & 5.19 & .023 \\
Profile 3 vs. 4 & 3.50 & $\mathbf{. 0 6 1}$ \\
Profile 3 vs. 5 & 8.80 & .003 \\
Profile 3 vs. 6 & 6.80 & .009 \\
Profile 4 vs. 5 & 14.44 & $<.001$ \\
Profile 4 vs. 6 & 15.51 & $<.001$ \\
Profile 5 vs. 6 & 1.95 &. $\mathbf{1 6 2}$ \\
\hline
\end{tabular}

Note. Time 2: Profile 1 = Low risk/low strength, Profile $2=$ Aggressive, complex need/low strength, Profile $3=$ Moderate risk/moderate strength, Profile $4=$ Low risk/high strength, and Profile $5=$ Low stability, complex need/low strength. Time 3: Profile $1=$ Low risk/low strength, Profile $2=$ Aggressive, complex need/low strength, Profile $3=$ Moderate risk/moderate strength, Profile $4=$ Low risk/high strength, Profile $5=$ Low stability, complex need/low strength, and Profile $6=$ Moderate complex need/low strength. $\chi^{2}=$ Chi-square pairwise test. 


\section{Appendix K}

\section{Supplementary LPA Analyses - Results for Full Sample of Men and Women from 3- Year Fixed-Follow-up Cohort with Initial SPIn Full Assessment}

In order to examine profile differences between the sample included in the main analyses (1,645 women and 6,675 men) and the full sample of men and women, an LPA was conducted for the entire sample of men and women with an initial full SPIn assessment conducted within 90 days of the start of supervision and who have recidivism information. This was done to examine whether similar profiles structures emerged when those who recidivated prior to receiving three assessments were included in the analyses (or whether there are additional unique profiles that emerge when these individuals are included in the analyses). The analyses included 16,605 men and 3,895 women. Due to the small number of women, relative to the number of men, LPAs were ran independently for men and women to account for any gender differences that would otherwise be masked by the large sample of men.

Various profile model solutions were run sequentially (ranging from 2 to 8 classes) to identify the best fitting model for men and women, independently (see Table $1 \mathrm{~K})$. Upon considering the model fit indices, theory, and the interpretation of the various profiles, it was determined that a 3-profile solution fit the data best for women, whereas a 5-profile solution fit the data the best for men. Notably, the 5-profile solution was chosen for men because structures containing any more profiles than this started to become less distinct, with differences that were not meaningful for interpretation purposes. 
Table $1 \mathrm{~K}$

Relative Fit Statistics for Men and Women

\begin{tabular}{|c|c|c|c|c|c|c|}
\hline \multicolumn{7}{|c|}{ Women } \\
\hline Profile & AIC & BIC & ABIC & Entropy & LMR & $p$ \\
\hline 2-Structure & 324953 & 325297 & 325123 & .875 & 13573.25 & $<.001$ \\
\hline 3-Structure & 319169 & 319645 & 319403 & .900 & 5792.77 & $<.001$ \\
\hline 4-Structure & 316500 & 317108 & 316800 & .868 & 2694.92 & $<.001$ \\
\hline 5-Structure & 314807 & 315546 & 315172 & .841 & 1725.21 & .002 \\
\hline 6-Structure & 312568 & 313440 & 312998 & .896 & 1882.43 & .072 \\
\hline 7-Structure & 312402 & 313404 & 312896 & .869 & 207.71 & .794 \\
\hline 8-Structure & 309905 & 311039 & 310464 & .866 & 1076.65 & .086 \\
\hline \multicolumn{7}{|c|}{ Men } \\
\hline Profile & AIC & BIC & $\overline{A B I C}$ & Entropy & LMR & $p$ \\
\hline 2-Structure & 1398422 & 1398862 & 1398681 & .883 & 54194.60 & $<.001$ \\
\hline 3-Structure & 1356186 & 1356773 & 1356531 & .887 & 24669.52 & $<.001$ \\
\hline 4-Structure & 1342840 & 1343589 & 1343281 & .889 & 13322.66 & $<.001$ \\
\hline 5-Structure & 1334666 & 1335576 & 1335201 & .907 & 8176.62 & $<.001$ \\
\hline 6-Structure & 1325948 & 1327021 & 1326579 & .889 & 7772.90 & $<.001$ \\
\hline 7-Structure & 1318940 & 1320175 & 1319666 & .868 & 7015.91 & $<.001$ \\
\hline 8-Structure & 1314291 & 1315688 & 1315113 & .857 & 4667.45 & .003 \\
\hline
\end{tabular}

Note. AIC = Akaike's Information Criterion. BIC = Bayesian Information Criteria. ABIC $=$ Sample size adjusted Bayesian Information Criteria. LMR $=$ Lo-Mendell-Rubin test.

Posterior profile membership probabilities were also examined (see Table $2 \mathrm{~K}$ ).

The probabilities of correct classification for the three profiles that emerged for women ranged from .95 to .97 , and for the five profiles that emerged for men ranged from .93 to .97 , which is considered good. 
Table $2 \mathrm{~K}$

Posterior Profile Membership Probabilities for Women and Men

\begin{tabular}{cccccc}
\hline & \multicolumn{5}{c}{ Women } \\
\hline Most Likely Profile & \multicolumn{5}{c}{ Latent Profiles } \\
\cline { 2 - 6 } Membership & 1 & 2 & 3 \\
\hline 1 & .966 & .034 & .000 \\
3 & .010 & .955 & .035 & \\
\hline & .000 & .048 & .952 & \\
\hline Most Likely Profile & & Men & & \\
Membership & 1 & 2 & 3 & .000 \\
1 & .955 & .015 & .010 & .020 & .000 \\
2 & .010 & .927 & .001 & .062 & .000 \\
3 & .025 & .002 & .973 & .000 & .042 \\
4 & .003 & .019 & .000 & .936 & .066 \\
5 & .000 & .000 & .000 & .934 \\
\hline
\end{tabular}

Note. Probability of class membership classification equal to or greater than .70 is considered acceptable.

Profile descriptions. The estimated means and standard deviations for each of the profiles were identified and labelled for women (see Table $3 \mathrm{~K}$ and Figure $1 \mathrm{~K}$ ) and men (see Table $4 \mathrm{~K}$ and Figure $2 \mathrm{~K}$ ). 
Table 3K

Means for Each of the Domains across the Three Profiles of Women

\begin{tabular}{|c|c|c|c|c|c|c|}
\hline \multirow[b]{2}{*}{ Variables in LPA } & \multicolumn{2}{|c|}{$\begin{array}{l}\text { Profile } 1 \\
(n=421)\end{array}$} & \multicolumn{2}{|c|}{$\begin{array}{c}\text { Profile 2 } \\
(n=1,979)\end{array}$} & \multicolumn{2}{|c|}{$\begin{array}{l}\text { Profile } 3 \\
(n=1,495)\end{array}$} \\
\hline & $M$ & $S D$ & $M$ & $S D$ & $M$ & $S D$ \\
\hline Criminal history & 8.12 & $(5.34)$ & 6.90 & $(5.07)$ & 3.49 & $(3.83)$ \\
\hline Aggression $^{1}-$ Risk & 2.04 & $(1.93)$ & 0.26 & $(0.68)$ & 0.06 & $(0.37)$ \\
\hline Aggression - Strength & 0.72 & $(1.20)$ & 2.24 & $(1.67)$ & 4.24 & $(1.76)$ \\
\hline Substance use - Risk & 12.69 & $(14.19)$ & 8.90 & $(9.01)$ & 5.06 & $(6.90)$ \\
\hline Social influence - Risk & 8.25 & $(4.94)$ & 4.78 & $(3.46)$ & 1.70 & $(1.94)$ \\
\hline Social influence - Strength & 2.01 & $(2.30)$ & 3.54 & $(2.51)$ & 7.56 & $(3.13)$ \\
\hline Family - Risk & 8.79 & $(5.35)$ & 5.56 & $(4.24)$ & 3.25 & $(3.28)$ \\
\hline Family - Strength & 2.00 & $(1.72)$ & 3.29 & $(2.03)$ & 6.13 & $(2.78)$ \\
\hline Employment - Risk & 5.48 & $(3.62)$ & 2.06 & $(2.56)$ & 0.42 & $(1.15)$ \\
\hline Employment - Strength & 0.69 & $(1.37)$ & 1.67 & $(1.84)$ & 4.59 & $(3.01)$ \\
\hline Attitudes - Risk & 4.31 & $(2.93)$ & 1.09 & $(1.55)$ & 0.36 & $(0.91)$ \\
\hline Attitudes - Strength & 2.20 & $(2.43)$ & 6.14 & $(3.52)$ & 11.07 & $(3.95)$ \\
\hline Skills $^{2}-$ Risk & 5.34 & $(2.73)$ & 0.78 & (1.19) & 0.13 & $(0.53)$ \\
\hline Skills - Strength & 0.50 & $(0.98)$ & 3.01 & $(2.79)$ & 8.40 & $(3.75)$ \\
\hline Stability - Risk & 4.91 & $(2.84)$ & 2.95 & $(2.16)$ & 1.15 & $(1.51)$ \\
\hline Stability - Strength & 2.11 & $(1.69)$ & 3.15 & $(1.56)$ & 4.87 & $(1.27)$ \\
\hline Mental health - Flag & 1.57 & $(0.76)$ & 1.27 & $(0.87)$ & 0.95 & $(0.90)$ \\
\hline $\mathrm{ACEs}^{3}$ & 2.91 & (1.89) & 2.44 & $(1.79)$ & 1.39 & $(1.55)$ \\
\hline
\end{tabular}

Note. $M=$ Mean, $S D=$ Standard deviation. Profile $1=$ Aggressive, complex needs/low strength, Profile $2=$ Moderate complex need/moderate strength, Profile $3=$ Low risk/high strength. ${ }^{1}$ Aggression refers to the aggression/violence domain. ${ }^{2}$ Skills refers to the cognitive/social skills domain. ${ }^{3} \mathrm{ACEs}$ refers to adverse childhood experiences. 


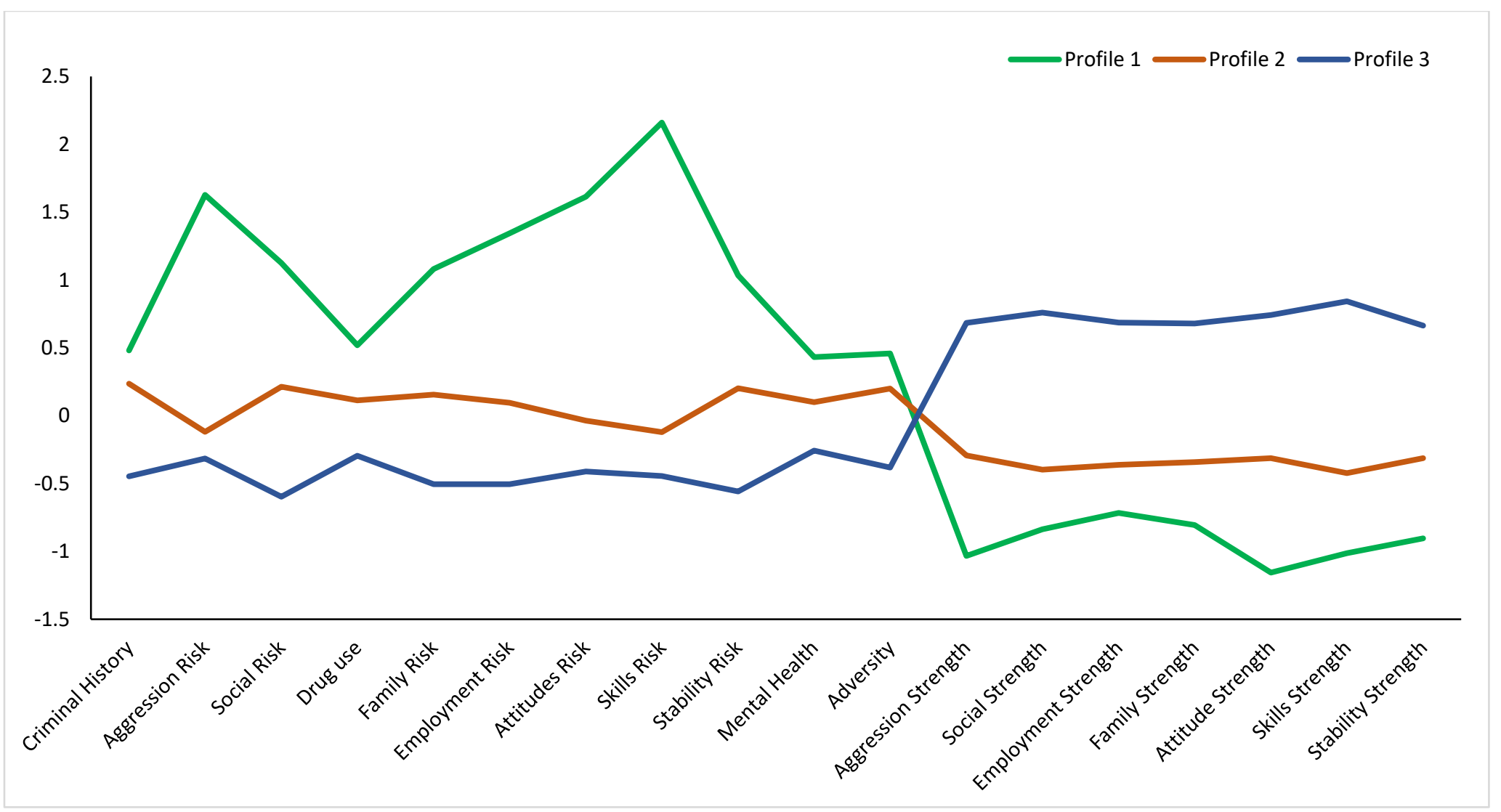

Figure $1 K$. Standardized domain scores for the three profiles for women. 
The following three profiles emerged for women:

Profile 1: Aggressive, complex need/low strength. This profile makes up the smallest proportion of women $(n=421 ; 10.81 \%)$ and is characterized by highest scores across all domains (especially the skills need domain). This profile also scores extremely high on aggression/violence need and scores high on complex needs including family needs, mental health concerns, and adverse childhood experiences. This profile, however, scores lowest on all strengths.

Profile 2: Moderate complex need/moderate strength. This profile makes up about half of the women in the study $(n=1,979 ; 50.81 \%)$ and is characterized by scoring moderately on all domains, including mental health and negative childhood adversity, and all strength domains). This class also has no particular domain that is more problematic than another.

Profile 3: Low risk/high strength. This profile makes up over one-third of the women in the study $(n=1,495 ; 38.38 \%)$ and is characterized by scoring lowest on all need domains (including complex needs, such as mental health, family needs, and childhood adversity). This profile scores highest on all strengths. 
Table $4 \mathrm{~K}$

Means for each of the domains across the five profiles of men

\begin{tabular}{|c|c|c|c|c|c|c|c|c|c|c|}
\hline \multirow[b]{2}{*}{ Variables in LPA } & \multicolumn{2}{|c|}{$\begin{array}{c}\text { Profile 1 } \\
(n=1,264)\end{array}$} & \multicolumn{2}{|c|}{$\begin{array}{c}\text { Profile 2 } \\
(n=2,122)\end{array}$} & \multicolumn{2}{|c|}{$\begin{array}{l}\text { Profile } 3 \\
(n=467)\end{array}$} & \multicolumn{2}{|c|}{$\begin{array}{c}\text { Profile } 4 \\
(n=8,073)\end{array}$} & \multicolumn{2}{|c|}{$\begin{array}{c}\text { Profile 5 } \\
(n=4,679)\end{array}$} \\
\hline & $M$ & $S D$ & $M$ & $S D$ & $M$ & $S D$ & $M$ & $S D$ & $M$ & $S D$ \\
\hline Criminal history & 9.50 & $(5.76)$ & 11.12 & $(5.36)$ & 12.20 & $(5.53)$ & 8.29 & $(5.50)$ & 5.58 & $(5.01)$ \\
\hline Aggression $^{1}-$ Risk & 2.99 & $(0.92)$ & 0.39 & $(0.63)$ & 5.03 & $(1.50)$ & 0.11 & $(0.35)$ & 0.03 & $(0.20)$ \\
\hline Aggression - Strength & 0.21 & $(0.52)$ & 1.24 & $(1.42)$ & 0.03 & $(0.21)$ & 2.21 & $(1.60)$ & 4.18 & $(1.59)$ \\
\hline Substances - Risk & 9.46 & $(9.47)$ & 12.20 & $(11.43)$ & 13.08 & $(14.28)$ & 7.63 & $(7.65)$ & 5.70 & $(7.11)$ \\
\hline Social $^{2}$ - Risk & 5.75 & $(4.38)$ & 7.57 & $(4.67)$ & 9.68 & $(5.89)$ & 3.73 & $(2.96)$ & 1.72 & $(2.08)$ \\
\hline Social - Strength & 3.35 & $(2.88)$ & 2.11 & $(2.22)$ & 1.58 & $(2.30)$ & 4.23 & $(2.74)$ & 7.86 & $(3.11)$ \\
\hline Family - Risk & 7.68 & $(5.16)$ & 6.36 & $(4.73)$ & 9.06 & $(6.13)$ & 4.78 & $(4.05)$ & 2.92 & $(3.24)$ \\
\hline Family - Strength & 3.66 & $(2.19)$ & 2.26 & $(1.84)$ & 1.88 & $(1.69)$ & 3.74 & $(2.21)$ & 6.12 & $(2.90)$ \\
\hline Employ - Risk & 0.98 & $(1.72)$ & 3.60 & $(3.08)$ & 4.54 & $(3.87)$ & 0.43 & $(1.00)$ & 0.14 & $(0.57)$ \\
\hline Employ - Strength & 3.37 & $(2.80)$ & 1.07 & $(1.47)$ & 1.41 & $(2.24)$ & 3.63 & $(2.52)$ & 6.42 & $(3.07)$ \\
\hline Attitudes - Risk & 3.60 & $(2.91)$ & 2.75 & $(2.55)$ & 8.01 & $(3.99)$ & 1.33 & $(1.79)$ & 0.37 & $(0.93)$ \\
\hline Attitudes - Strength & 3.05 & $(3.11)$ & 3.08 & $(3.06)$ & 0.80 & $(1.77)$ & 5.40 & $(3.33)$ & 10.85 & $(3.71)$ \\
\hline Skills $^{3}-$ Risk & 3.26 & $(2.52)$ & 2.92 & $(2.58)$ & 8.20 & $(3.23)$ & 0.71 & $(1.30)$ & 0.09 & $(0.39)$ \\
\hline Skills - Strength & 1.31 & $(1.87)$ & 1.18 & $(1.78)$ & 0.24 & $(0.65)$ & 3.15 & $(2.70)$ & 8.92 & $(3.35)$ \\
\hline Stability - Risk & 2.19 & $(2.14)$ & 5.05 & $(2.38)$ & 4.44 & $(3.18)$ & 1.45 & $(1.61)$ & 0.74 & $(1.21)$ \\
\hline Stability - Strength & 3.80 & $(1.80)$ & 1.79 & $(1.39)$ & 2.45 & (1.94) & 4.20 & $(1.48)$ & 5.24 & $(1.29)$ \\
\hline Mental health & 1.06 & $(0.93)$ & 1.26 & $(0.90)$ & 1.47 & $(0.84)$ & 0.79 & $(0.90)$ & 0.53 & $(0.80)$ \\
\hline $\mathrm{ACEs}^{4}$ & 1.74 & $(1.59)$ & 2.08 & $(1.60)$ & 2.20 & $(1.79)$ & 1.29 & $(1.38)$ & 0.81 & $(1.16)$ \\
\hline
\end{tabular}

Note. $M=$ Mean, $S D=$ Standard deviation. Profile 1: Aggressive, moderate complex need/low strength, Profile $2=$ Low stability, complex needs/low strength, Profile 3: Aggressive, high complex needs/low strength, Profile $4=$ Moderate risk/moderate strength, Profile $5=$ Low risk/high strength.

${ }^{1}$ Aggression refers to the aggression/violence domain. ${ }^{2}$ Social refers to social influences domain. ${ }^{3}$ Skills refers to the cognitive/social skills domain. ${ }^{4} \mathrm{ACEs}$ refers to adverse childhood experiences. 


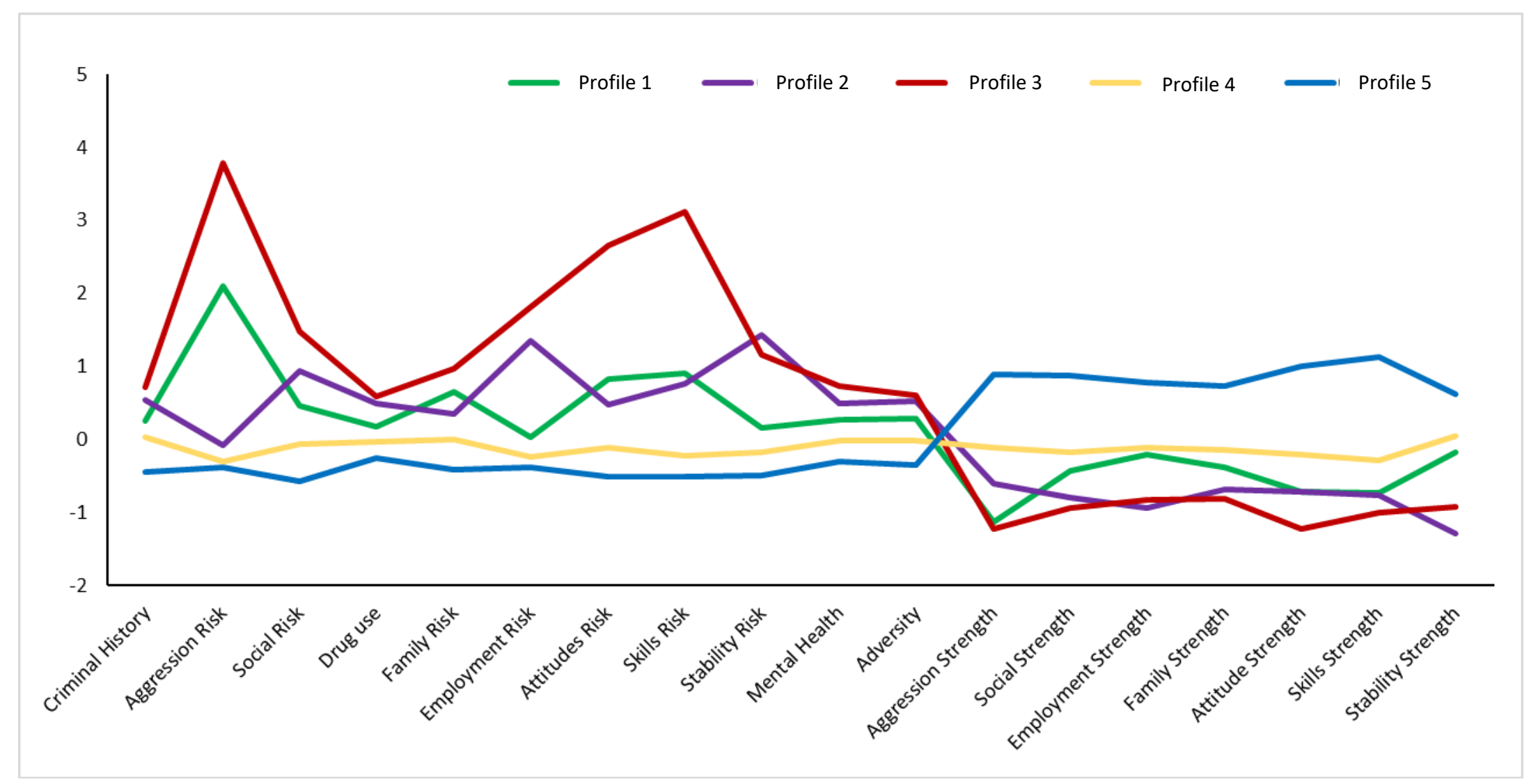

Figure $2 K$. Standardized domain scores for the five profiles of men. 
The following five profiles emerged for men:

Profile 1: Aggressive, moderate complex need/low strength. This profile makes up $7.61 \%$ of men $(n=1,264)$ and is characterized as scoring high on the aggressive need domain and moderate to high on all other need domains (including mental health and childhood adversity). This profile also scores low to moderate on all strength domains.

Profile 2: Low stability, complex needs/low strength. This profile makes up $12.78 \%$ of the men $(n=2,122)$ and is characterized as scoring moderately on most needs, with the exception of stability, whereby individuals in this profile tend to score the highest. This profile is non-aggressive, and tend to score higher on mental health needs and childhood adversity, and tend to score low to moderate on all strength domains.

Profile 3: Aggressive, high complex needs/low strength. This profile makes up the smallest proportion of men $(n=467 ; 2.81 \%)$ and is characterized as being extremely aggressive and violent with high antisocial attitudes. This profile scores highest on most need domains, including complex needs such as mental health and childhood adversity. This profile scores lowest on most strength domains.

Profile 4: Moderate risk/moderate strength. This profile makes up just under half of the men sample $(n=8,073 ; 48.62 \%)$ and is characterized by scoring similarly on strength and need domains (with no needs or strengths that are more problematic than others). Individuals in this profile do not score the lowest or highest on any risk or strength domains.

Profile 5: Low risk/high strength. This profile makes up just over a quarter of the sample $(n=4,679 ; 28.18 \%)$ and is characterized by scoring the lowest on all need 
domains (including complex needs, such as mental health and childhood adversity), but score the highest on all strength domains.

\section{Criminal Outcome for Women}

Distal outcomes were examined in relation to profile structure. The distal outcomes included were technical violations, any new charges, violent charges, and any negative outcome. The proportions of each profile that experienced the various criminal outcomes are presented in Table $5 \mathrm{~K}$.

Table 5K

Proportions of Women in Each Profile who Displayed Criminal Outcomes

\begin{tabular}{lcccccccc}
\hline & \multicolumn{2}{c}{ TV } & \multicolumn{2}{c}{ Any New } & \multicolumn{2}{c}{ Violent } & \multicolumn{2}{c}{ Negative outcome } \\
\cline { 2 - 9 } Profile & $\boldsymbol{n}$ & $\mathbf{\%}$ & $\boldsymbol{n}$ & $\mathbf{\%}$ & $\boldsymbol{n}$ & $\mathbf{\%}$ & $\boldsymbol{n}$ & $\%$ \\
\hline Profile 1 & 154 & 36.6 & 142 & 33.7 & 69 & 16.4 & 198 & 47.0 \\
Profile 2 & 549 & 27.7 & 604 & 30.5 & 245 & 12.4 & 819 & 41.4 \\
Profile 3 & 153 & 10.2 & 217 & 14.5 & 57 & 3.8 & 275 & 18.4 \\
\hline
\end{tabular}

Note. $\mathrm{TV}=$ Technical violations. Profile sizes: Profile 1 (Aggressive, complex need/low strength $)=421$; Profile 2 (Moderate complex need/ moderate strength $)=1$, 979; Profile 3 (Low risk/high strength) $=1,495$.

Not surprisingly, Profile 1 (Aggressive, complex need/low strength) had the highest proportion of all four types of criminal outcomes. Profile 3 (Low risk/high strength) reported the lowest proportions of each type of criminal outcome, which is also demonstrated in Table 6K, where Profile 3 had significantly lower proportions of each type of criminal outcome than the other two profiles. 
Table $6 \mathrm{~K}$

Women's Profile Comparisons of Proportions of Various Criminal Outcome

\begin{tabular}{|c|c|c|}
\hline Technical Violation & Odds Ratio & $p$ \\
\hline Profile 1 to Profile 2 & 1.499 & .005 \\
\hline Profile 1 to Profile 3 & 5.086 & $<.001$ \\
\hline Profile 2 to Profile 3 & 3.393 & $<.001$ \\
\hline Any new charge & Odds Ratio & $p$ \\
\hline Profile 1 to Profile 2 & 1.169 & .225 \\
\hline Profile 1 to Profile 3 & 3.034 & $<.001$ \\
\hline Profile 2 to Profile 3 & 2.595 & $<.001$ \\
\hline Any negative outcome & Odds Ratio & $p$ \\
\hline Profile 1 to Profile 2 & 1.258 & .069 \\
\hline Profile 1 to Profile 3 & 3.954 & $<.001$ \\
\hline Profile 2 to Profile 3 & 3.144 & $<.001$ \\
\hline Violent charge & Odds Ratio & $p$ \\
\hline Profile 1 to Profile 2 & 1.413 & .059 \\
\hline Profile 1 to Profile 3 & 5.095 & $<.001$ \\
\hline Profile 2 to Profile 3 & 3.606 & $<.001$ \\
\hline
\end{tabular}

Note. Profile 1 = Aggressive, complex needs/low strength; Profile 2 = Moderate complex need/ moderate strength; Profile 3 = Low risk/high strength.

\section{Criminal Outcome for Men}

The proportions of each profile for men that experienced the various criminal outcomes are presented in Table 7K. Overall, Profile 2 (Complex need/low strength) and Profile 3 (Aggressive, high complex need/low strength) had the highest proportion of each criminal outcome. 
Table $7 \mathrm{~K}$

Proportions of Men in each Profile who Displayed Criminal Outcomes

\begin{tabular}{lcccccccc}
\hline & \multicolumn{2}{c}{ TV } & \multicolumn{2}{c}{ Any New } & \multicolumn{2}{c}{ Violent } & \multicolumn{2}{c}{ Negative outcome } \\
\cline { 2 - 8 } Profile & $\boldsymbol{n}$ & $\%$ & $\boldsymbol{n}$ & $\%$ & $\boldsymbol{n}$ & $\%$ & $\boldsymbol{n}$ & $\%$ \\
\hline Profile 1 & 397 & 31.4 & 431 & 34.1 & 281 & 22.2 & 524 & 41.5 \\
Profile 2 & 974 & 45.9 & 986 & 46.5 & 482 & 22.7 & 1,300 & 61.3 \\
Profile 3 & 226 & 48.4 & 223 & 47.8 & 150 & 32.1 & 296 & 63.4 \\
Profile 4 & 1,914 & 23.7 & 2,381 & 29.5 & 1,301 & 16.1 & 2,975 & 36.9 \\
Profile 5 & 546 & 11.7 & 850 & 18.2 & 395 & 8.4 & 1,050 & 22.4 \\
\hline
\end{tabular}

Note. $\mathrm{TV}=$ Technical violations. Profile sizes: Profile 1 (Aggressive, moderate complex need/low strength $)=1,264$, Profile 2 (Low stability, complex need/low strength $)=2,122$, Profile 3 (Aggressive, high complex need/low strength) $=467$, Profile 4 (Moderate risk/moderate strength $)=8,073$, Profile $5($ Low risk/high strength $)=4,679$.

Odds ratios were used to compare the various profiles in terms of proportions of various criminal outcomes (see Table $8 \mathrm{~K}$ ). Across all four criminal outcomes, Profile 2 (Low stability, complex need/low strength) and Profile 3 (Aggressive, high complex need/low strength) did not significantly differ in terms of proportions of technical violations, new charges, and any negative outcome. Similarly, Profile 1 (Aggressive, moderate complex need/low strength) and Profile 2 (Low stability, complex need/low strength) did not significantly differ in terms of proportions of men who were charged for a violent offence. 
Table $8 \mathrm{~K}$

Men's Profile Comparisons of Proportions of Various Criminal Outcomes

\begin{tabular}{lccccccccc}
\hline & \multicolumn{2}{c}{ Technical violation } & \multicolumn{2}{c}{ Any new charge } & \multicolumn{2}{c}{ Violent charge } & \multicolumn{2}{c}{ Negative outcome } \\
\cline { 2 - 9 } Profile comparisons & OR & $\boldsymbol{p}$ & OR & $\boldsymbol{p}$ & OR & $\boldsymbol{p}$ & OR & \multicolumn{1}{c}{$\boldsymbol{p}$} \\
\hline Profile 1 to Profile 2 & 0.555 & $<.001$ & .597 & $<.001$ & 0.958 & .631 & 0.461 & $<.001$ \\
Profile 1 to Profile 3 & 0.490 & $<.001$ & .556 & $<.001$ & 0.611 & $<.001$ & 0.407 & $<.001$ \\
Profile 1 to Profile 4 & 1.481 & $<.001$ & 1.219 & .008 & 1.495 & $<.001$ & 1.212 & .008 \\
Profile 1 to Profile 5 & 3.457 & $<.001$ & 2.322 & $<.001$ & 3.136 & $<.001$ & 2.449 & $<.001$ \\
Profile 2 to Profile 3 & 0.884 & .215 & 0.930 & .475 & 0.637 & $<.001$ & 0.883 & .228 \\
Profile 2 to Profile 4 & 2.670 & $<.001$ & 2.041 & $<.001$ & 1.560 & $<.001$ & 2.630 & $<.001$ \\
Profile 2 to Profile 5 & 6.233 & $<.001$ & 3.886 & $<.001$ & 3.272 & $<.001$ & 5.315 & $<.001$ \\
Profile 3 to Profile 4 & 3.020 & $<.001$ & 2.195 & $<.001$ & 2.448 & $<.001$ & 2.979 & $<.001$ \\
Profile 3 to Profile 5 & 7.050 & $<.001$ & 4.179 & $<.001$ & 5.135 & $<.001$ & 6.020 & $<.001$ \\
Profile 4 to Profile 5 & 2.335 & $<.001$ & 1.904 & $<.001$ & 2.098 & $<.001$ & 2.021 & $<.001$ \\
\hline
\end{tabular}

Note. OR $=$ Odds Ratio. Profile 1 = Aggressive, moderate complex need/low strength; Profile 2 = Low stability, complex need/low strength; Profile $3=$ Aggressive, high complex need/low strength; Profile $4=$ Moderate risk/moderate strength; Profile $5=$ Low risk/high strength. 